\title{
GALAXY EVOLUTION IN COMPACT GROUPS
}

\author{
Lisa May Walker \\ Rockville, MD
}

B.A. in Physics, College of Wooster, 2007

M.S. in Astronomy, University of Virginia, 2009

A Dissertation Presented to the Graduate

Faculty of the University of Virginia

in Candidacy for the Degree of

Doctor of Philosophy

\author{
Department of Astronomy \\ University of Virginia \\ May, 2014
}

Kelsey Johnson

Aaron Evans

Robert O'Connell

John Yates

Sarah Gallagher 
(C)Copyright by

Lisa May Walker

All rights reserved

May, 2014 
Abstract 
The processes by which galaxies formed is one of the most important open questions in astrophysics. Most generally, it is now thought that galaxies formed through "hierarchical formation," where galaxies grow by merging with similarly-sized galaxies and accreting smaller galaxies. Subsequently, clusters formed when galaxies would accumulate in gravitational potential wells. However, the detailed astrophysical processes involved are poorly constrained. Progress has been largely limited due to the lack of suitably high sensitivity and resolution data sets of these high redshift systems. In the absence of detailed observations of these environments in the earlier universe, the best current path forward is through relatively nearby analogs. Compact groups of galaxies provide a nearby environment with conditions similar to those in the earlier universe when galaxies were assembled. In particular, these groups give us the opportunity to witness hierarchical formation in progress. My studies of compact groups aim to determine how this intense environment - one of high galaxy density and constant interactions - affects the evolution of member galaxies. I compare the mid-IR colorspace distribution of compact group galaxies to several other environments, and find that the compact group galaxies occupy mid-IR colorspace in a unique way; there is a statistically significant canyon between quiescent galaxies and active galaxies not seen in a field sample, interacting pairs, or the center of Coma. However, the infall region of Coma shows a similar colorspace distribution, indicating similar environments; both have high densities and still contain neutral gas. An analysis of compact group galaxies' SEDs reveals that galaxies in different regions of mid-IR colorspace contain dust with varying properties. I compare mid-IR and optical colors of compact group galaxies and find that the mid-IR 'canyon' galaxies do not occupy the optical 'green valley' as expected, rather they fall on the optical 'red sequence', indicating that the mid-IR properties transition on a different timescale than the optical prop- 
erties. I obtain H I observations of Redshift Survey compact groups and place them in context with those of Hickson compact groups as well as compare with mid-IR and optical properties of member galaxies. 
Acknowledgements 


\section{Funding and Data Acknowledgements}

I have received funding from a variety of sources, including a UVa Presidential Graduate Research Fellowship and a VSGC Graduate Research fellowship. Support for this work was provided by the NSF through award GSSP 10A-003 from the NRAO. Further support has been provided by the David and Lucile Packard Foundation through a Packard Fellowship.

This thesis has made use of data from a number of excellent telescopes. This work is based in part on observations made with the Spitzer Space Telescope, which is operated by the Jet Propulsion Laboratory, California Institute of Technology under a contract with NASA. This publication makes use of data products from the Two Micron All Sky Survey, which is a joint project of the University of Massachusetts and the Infrared Processing and Analysis Center/California Institute of Technology, funded by the National Aeronautics and Space Administration and the National Science Foundation. This research has made use of the NASA/ IPAC Infrared Science Archive, which is operated by the Jet Propulsion Laboratory, California Institute of Technology, under contract with the National Aeronautics and Space Administration. This study also utilizes data from the National Radio Astronomy Observatory's Green Bank Telescope. The National Radio Astronomy Observatory is a facility of the National Science Foundation operated under cooperative agreement by Associated Universities, Inc. This dissertation also includes data from the Sloan Digital Sky Survey. Funding for the Sloan Digital Sky Survey (SDSS) has been provided by the Alfred P. Sloan Foundation, the Participating Institutions, the National Aeronautics and Space Administration, the National Science Foundation, the U.S. Department of Energy, the Japanese Monbukagakusho, and the Max Planck Society. The SDSS Web site is http://www.sdss.org/. 
The SDSS is managed by the Astrophysical Research Consortium (ARC) for the Participating Institutions. The Participating Institutions are The University of Chicago, Fermilab, the Institute for Advanced Study, the Japan Participation Group, The Johns Hopkins University, Los Alamos National Laboratory, the Max-PlanckInstitute for Astronomy (MPIA), the Max-Planck-Institute for Astrophysics (MPA), New Mexico State University, University of Pittsburgh, Princeton University, the United States Naval Observatory, and the University of Washington.

And of course, what research occurs without the use of NED and ADS? I know I certainly used these two resources extensively, thus: This research has made use of the NASA/IPAC Extragalactic Database (NED) which is operated by the Jet Propulsion Laboratory, California Institute of Technology, under contract with the National Aeronautics and Space Administration. This research has made use of NASA's Astrophysics Data System.

\section{Personal Acknowledgements}

There are two women without whom this thesis never would have happened. Kelsey, thank you for being my mentor these past 7 years. You've taught me how to do research, helped me realize that I won't always be able to do everything perfectly, and pushed me to branch out of my comfort zone into teaching, mentoring, and outreach. Mama, you made me take my first physics class, encouraged and supported me throughout college and grad school, and have always been there whenever I've needed you. I don't know how to thank you.

I also would like to thank my thesis committee for their support, advice, and guidance over the years, and for never giving up on me. I've also been surrounded by a fantastic group of collaborators, the Compact Groupies, who not only improved 
my research but made it more fun as well.

Before I came to UVa, I benefited from knowing some pretty amazing people. I'm so grateful for the mentoring of Tony Morgan and Robert Boyle, two of the most supportive and encouraging professors I've ever met. I never would have gotten here without the professors who guided my undergraduate research; Lowell Boone, Jack Baldwin, and of course my undergraduate thesis advisor, John Lindner. Julia, thank you for not letting me spend all my time at Tome. Mandy, my roomie, thank you for wanting to see me so much that you brought food (and Dr Pepper!) to the physics building. Kirsten, thank you for being the other astro-kid with me.

Kim, you're such a great officie. I'm glad we've been able to vent to each other, support each other, and make fun of each other. As Bo pointed out, we're good sisters. Natalie, thank you for being my first student. You survived my learning how to mentor, and never lost hope (even on the day that I told you that I'd taught you to code all wrong); if you can do that you'll do great in astronomy. Catherine, you've been so much fun to have as a student. It's been amazing watching you go from being a history major, reluctant to try research in astronomy, to double-majoring with astrophysics and plans to pursue this in graduate school. Someday I'll forgive you for waiting 7 months to tell me about your boyfriend. Janice, you do so much, both for the department and for me. You're so much fun to talk to that it's always hard to stop! I hope we're near each other next year.

To all the other UVa grads, before and after me. I can't imagine what grad school would have been like without you. To my older grads, who I miss constantly - Gail, Dan, Gevie (Dr. Pants!), Jeff Carlin, Joleen, Jarron (I can't wait to work with you), Nicole, Paul, George, Sela, Steph, and of course Jake 1.0 (moo point!). To my classmates - Abel, my "twin" David, and Rachael and George (can you believe 
we've been here 7 years?). To my younger grads - Bill (sketchy but harmless), Lauren (thank you for putting up with me this year!), Trey, Loreto, Patrick, Sandy (I'll miss our baking nights), Dom, Brian, Andrew, Tuck, and Scott.

Jake. You kept me sane, but didn’t mind that I sometimes wasn’t. Thank you for laughing with me, crying with me, and for just being there. Thank you for being a "crazy cat lady" with me, and not minding that I turned you into one. I love our cat conversations (I'm pretty sure that's like butter too), our cat songs, our cat snuggles, and laughing at the cats (remember when Maisie fell off the futon?). It's so much fun to laugh with you, relax with you (our pizza nap!), even driving on mountains is better with you (especially when you're the one driving!). 


\section{Table of contents}

Abstract iii

Acknowledgements vi vi

List of Figures $\quad$ Xv

List of Tables $\quad$ xvii

1 Introduction 1

1.1 Compact Groups of Galaxies . . . . . . . . . . . . . . . . . 2

1.2 Impact of Environment . . . . . . . . . . . . . . . . . . . . . 3

1.3 Compact Group Evolution . . . . . . . . . . . . . . 5

1.4 Properties of Compact Groups . . . . . . . . . . . . . . 5

1.5 A Multi-wavelength Approach . . . . . . . . . . . . . . . 6

1.6 Star Formation in Compact Groups . . . . . . . . . . . . . 7

1.7 Current Knowledge of the Cold Gas . . . . . . . . . . . . . . 8

1.8 Compact Group Catalogs . . . . . . . . . . . . . . . . . . . . . 10

1.8.1 Hickson Compact Groups . . . . . . . . . . . . . . . . . 11

1.8.2 Redshift Survey Compact Groups . . . . . . . . . . . . . . . 11

1.8 .3 Other Catalogs . . . . . . . . . . . . . . . . . . . . . 12

1.9 Organization of this Thesis . . . . . . . . . . . . . . 12

2 Mid-Infrared Evidence for Accelerated Evolution in Compact Group Galaxies 15

2.1 Introduction . . . . . . . . . . . . . . . . . . . 17

2.2 Data/Samples . . . . . . . . . . . . . . . . . . . . . . . . . . . . . . 19

2.2 .1 HCG Galaxies . . . . . . . . . . . . . . . . . . . . . . . . . 19

2.2 .2 Comparison Samples . . . . . . . . . . . . . . . . . . . . . . 23

2.3 Mid-Infrared Colorspace . . . . . . . . . . . . . . . . . . . . . 26

2.3 .1 HCG Galaxies . . . . . . . . . . . . . . . . . . . . . . . 26

2.3 .2 Comparison Samples . . . . . . . . . . . . . . . . . . . 28

2.4 Statistical Tests . . . . . . . . . . . . . . . . . . . . 30

2.4.1 Single-Distribution KS Test . . . . . . . . . . . . . 31 
2.4.2 Two-Distribution KS Test . . . . . . . . . . . . . . . 36

2.4.3 Gap Region . . . . . . . . . . . . . . . . . . 36

2.5 Color-Magnitude Diagrams . . . . . . . . . . . . . . . . . . . . . . . . . . . . . . . . . .

2.6 Properties of Galaxies along Mid-IR Colorspace . . . . . . . . . . . . 42

2.6.1 Morphology . . . . . . . . . . . . . . . . . . 42

2.6.2 EW $(\mathrm{H} \alpha)$ and Specific Star Formation Rates . . . . . . . . . 46

2.7 Discussion . . . . . . . . . . . . . . . . . . . . . . . . . . . 48

2.7.1 The relationship between environment and presence of gap . . 48

2.7.2 Possible mechanisms for Mid-IR gap . . . . . . . . . . . . . . 48

2.7.3 Relationship to optical CMDs . . . . . . . . . . . . . 50

2.7.4 The role of gas in CG evolution . . . . . . . . . . . . 51

3 Examining the Role of Environment in a Comprehensive Sample of Compact Groups $\quad 56$

3.1 Introduction . . . . . . . . . . . . . . . . . 58

3.2 Data . . . . . . . . . . . . . . . . . . 60

3.2.1 Samples .................... . . . 60

3.2.2 Observations . . . . . . . . . . . . . . . . . . . . 61

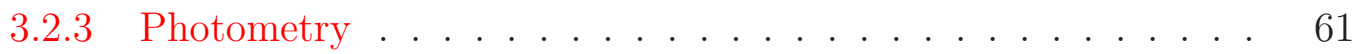

3.3 Colorspace . . . . . . . . . . . . . . . . . 66

3.3 .1 Full Sample . . . . . . . . . . . . . . . 66

3.3.2 Subsamples . . . . . . . . . . . . . . . . . . 70

3.3.3 Comparison Samples . . . . . . . . . . . . . . . 73

3.4 Color-Magnitude Diagrams . . . . . . . . . . . . . . . . . . . 75

3.5 Spectral Energy Distributions . . . . . . . . . . . . . . . . 79

3.6 Discussion . . . . . . . . . . . . . . . . . . . . . . . . . . 79

3.6.1 The Canyon . . . . . . . . . . . . . . . . 80

3.6.2 Impact of Subsamples . . . . . . . . . . . . . . . . . . . . 80

3.6.3 Environment . . . . . . . . . . . . . . . 81

3.6 .4 SEDs . . . . . . . . . . . . . . . . . 82

3.6.5 Conclusions . . . . . . . . . . . . . . . . 82

4 The Optical Green Valley vs Mid-IR Canyon in Compact Groups 83

4.1 Introduction . . . . . . . . . . . . . . . 85

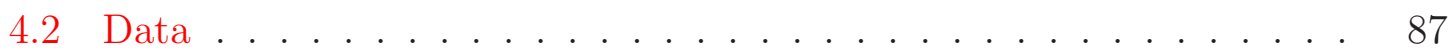

4.2.1 The Sample . . . . . . . . . . . . . . . . . . . 87

4.2.2 Completeness . . . . . . . . . . . . . . . . . . . 88

4.2 .3 Photometry . . . . . . . . . . . . . . . . . . 89

4.3 Results . . . . . . . . . . . . . . . . . . . . . . . . . . . . . . . . . . .

4.3.1 Color-Magnitude Diagram . . . . . . . . . . . . . . 90

4.3.2 Optical vs mid-IR . . . . . . . . . . . . . . . . . . . . . . . 93

4.3 .3 Stars vs Dust . . . . . . . . . . . . . . . . 94 
4.4 Discussion . . . . . . . . . . . . . . . . . . . . . . . . . . 97

4.4.1 Transition Galaxies in Optical and mid-IR . . . . . . . . . . . 97

4.4.2 Implications for Galaxy Evolution . . . . . . . . . . . . . . . . 98

5 Neutral Hydrogen and Galaxy Properties in Compact Groups 102

5.1 Introduction . . . . . . . . . . . . . . . . 104

5.2 Data . . . . . . . . . . . . . . . . . 105

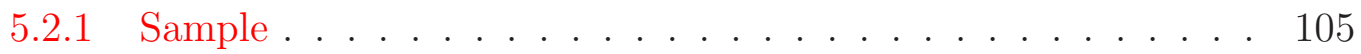

$5.2 .2 \mathrm{H}$ IData . . . . . . . . . . . . . . . . . . . . . . . . . . . . . . . . . . . . . . .

5.3 Results . . . . . . . . . . . . . . . . . . . . . 108

5.3.1 H IContent: Comparison with Stellar Masses . . . . . . . . . . 118

5.4 Discussion . . . . . . . . . . . . . . . . . . 119

5.4.1 Morphologies . . . . . . . . . . . . . . . . . 119

5.4 .2 Mid-IR and Optical Colors . . . . . . . . . . . . . . . . . 119

5.5 Conclusions . . . . . . . . . . . . . . . . . . 122

6 Summary and Future Work 125

6.1 Outstanding Questions . . . . . . . . . . . . . . 129

A Optical Photometry 132

B Acronyms 153 


\section{List of Figures}

1.1 Examples of different H I distributions in compact groups . . . . . . . 9

2.1 Luminosities of HCG galaxies and comparison samples . . . . . . . . 20

2.2 Morphologies of HCG and field galaxies . . . . . . . . . . . . . . . 21

2.3 Distribution of HCG galaxies in mid-IR colorspace . . . . . . . . . . . 27

2.4 mid-IR colorspace for comparison samples . . . . . . . . . . . . . 29

2.5 KS test for the HCG distribution against a uniform distribution . . . 32

2.6 KS test for the comparison samples against a uniform distribution . . 35

2.7 KS test for the comparison samples against the HCG galaxies . . . . 37

2.8 Mid-IR CMD for the HCG galaxies . . . . . . . . . . . . . . . . . . . 39

2.9 Mid-IR CMD for the comparison samples . . . . . . . . . . . . . . 41

2.10 Distribution of galaxy morphology in mid-IR colorspace . . . . . . . . 43

2.11 Distribution of galaxy morphology in rotated mid-IR colorspace . . . 45

2.12 Galaxies with optical spectra identified in mid-IR colorspace . . . . . 45

$2.13 E W(H \alpha)$ vs mid-IR color . . . . . . . . . . . . . . . . . . . . 54

2.14 Specific star formation against mid-IR color . . . . . . . . . . 55

3.1 Luminosities of compact group galaxies and comparison samples . . . 63

3.2 Morphologies of compact group and field galaxies . . . . . . . . . . . 64

3.3 Distribution of compact group galaxies in mid-IR colorspace . . . . . 68

3.4 Identification of the mid-IR canyon . . . . . . . . . . . . 69

3.5 KS test for compact group galaxies against a uniform distribution . . 71

3.6 Location of mid-IR canyon and comparison with original sample . . . 71

3.7 Effect of sample selection on mid-IR colorspace distribution . . . . . . 74

3.8 Effect of physical properties on colorspace distribution . . . . . . . . 74

3.9 mid-IR colorspace for comparison samples . . . . . . . . . . . . . 76

3.10 Mid-IR CMD for the compact group galaxies . . . . . . . . . . . . 77

3.11 Mid-IR CMD for the comparison samples . . . . . . . . . . . . . . . 77

3.12 Mid-IR CMD for the comparison samples . . . . . . . . . . . . . 78

3.13 Effect of dust on mid-IR colors . . . . . . . . . . . . . . . 78

4.1 Luminosities of compact group galaxies and comparison samples . . . 91

4.2 Comparison of photometry methods . . . . . . . . . . . . . . 91 
4.3 Optical color-magnitude diagrams of compact group galaxies and field galaxies . . . . . . . . . . . . . . . . . . . . . . 92

4.4 Histogram of optical colors for compact groups and comparison samples 95

4.5 Comparison of optical with mid-IR colors . . . . . . . . . . . . 95

4.6 Comparison of the amount of light emitted in the optical vs the mid-IR 96

4.7 Comparison of the $8.0 \mu \mathrm{m}$ and $4.5 \mu \mathrm{m}$ luminosities . . . . . . . . . . 101

5.1 H I spectrum and optical image for detected compact groups . . . . . 110

5.2 Comparison of $M_{H_{I}}$ and $M_{*}$ for compact groups . . . . . . . . . . . 120

5.3 Effect of sample selection on H I properties of compact groups . . . . 121

5.4 Effect of spiral fraction on H I properties of compact groups . . . . . 121

5.5 Group H I content and galaxy mid-IR colors . . . . . . . . . . . . . . 123

5.6 Group H I content and galaxy optical colors . . . . . . . . . . . . . . 124 


\section{List of Tables}

1.1 Catalogs of Compact Groups . . . . . . . . . . . . . . . . . . . 14

2.1 Properties of HCG Sample . . . . . . . . . . . . . . . . . . . . . . 24

2.2 Single-Distribution KS Test . . . . . . . . . . . . . . . . . . 34

2.3 Two-Distribution KS Test . . . . . . . . . . . . . . . . . . . 34

2.4 CDF Slopes in Gap Region . . . . . . . . . . . . . . . . 43

3.1 CG Sample . . . . . . . . . . . . . . . . . . . . . . . . 65

3.1 CG Sample . . . . . . . . . . . . . . . . . . . . . . . 67

3.2 Results of Statistical Analysis . . . . . . . . . . . . . . . 69

4.1 Galaxies in the mid-IR Canyon . . . . . . . . . . . . 96

5.1 Groups in H I Survey . . . . . . . . . . . . . . . . . . . 107

5.2 Masses and Upper Limits for RSCGs . . . . . . . . . . . . . 109

A.1 Photometry for HCG Galaxies . . . . . . . . . . . . . . . . 134

A.1 Photometry for HCG Galaxies . . . . . . . . . . . . . . 135

A.1 Photometry for HCG Galaxies . . . . . . . . . . . . . . 136

A.1 Photometry for HCG Galaxies . . . . . . . . . . . . . . 137

A.1 Photometry for HCG Galaxies . . . . . . . . . . . . . . 138

A.1 Photometry for HCG Galaxies . . . . . . . . . . . . . . . . . 139

A.1 Photometry for HCG Galaxies . . . . . . . . . . . . . . . . 140

A.1 Photometry for HCG Galaxies . . . . . . . . . . . . . . . 141

A.2 Photometry for RSCG Galaxies . . . . . . . . . . . . . . . 142

A.2 Photometry for RSCG Galaxies . . . . . . . . . . . . . . 143

A.2 Photometry for RSCG Galaxies . . . . . . . . . . . . . . 144

A.2 Photometry for RSCG Galaxies . . . . . . . . . . . . 145

A.2 Photometry for RSCG Galaxies . . . . . . . . . . . . . 146

A.2 Photometry for RSCG Galaxies . . . . . . . . . . . . . 147

A.2 Photometry for RSCG Galaxies . . . . . . . . . . . . . . 148

A.2 Photometry for RSCG Galaxies . . . . . . . . . . . . . . . 149

A.2 Photometry for RSCG Galaxies . . . . . . . . . . . . 150

A.2 Photometry for RSCG Galaxies . . . . . . . . . . . . . 151 
A.2 Photometry for RSCG Galaxies . . . . . . . . . . . . . . . . 152

B.1 Acronyms ......................... 154 
Chapter 1

\section{Introduction}


How did we get here? This is a question that, in some form, has fascinated humans for thousands of years. Initially, the question of where we came from only applied to humans, but as our understanding of the world around us has grown, so has the extent of this question. We used to believe that Earth was the center of the universe, but when we realized that Earth is actually one of several planets orbiting the sun, the question became where our solar system came from. When we discerned that our star was one of millions making up a galaxy, or "island universe," we began to wonder not only where it came from but how big it was. Eventually, we came to understand that our galaxy is part of a cosmic web of galaxies that make up the universe. We now know that everything around us, everything there is, started in one Big Bang. Now the question is how we got from the Big Bang to the world and universe we live in today.

\subsection{Compact Groups of Galaxies}

The processes by which galaxies form is one of the most important open issues in astrophysics. It is thought that galaxies and clusters grow by mergers and accretion via "hierarchical formation." In fact, the majority of galaxies spend most of their life in a group of some kind (Mulchaey 2000), making groups an important site in understanding the growth of structure. The Hubble Ultra Deep Field has revealed that in the early universe, the evolution of galaxies was influenced by multiple interactions (Weinzirl et al. 2011; Cooper et al. 2012). As a nearby environment of galaxy assembly, compact groups - extremely dense associations of three or more galaxies (e.g., HCGs; Hickson 1982; Hickson et al. 1992) - present an opportunity to witness hierarchical formation in progress. I aim to determine how this environment of high galaxy density and frequent, prolonged interactions affects the evolution of the mem- 
ber galaxies. Our research with Spitzer has already found clear evidence that galaxies in compact groups evolve differently than galaxies in other environments, but it is not yet fully understood why.

While interacting pairs and galaxy clusters have been well-studied, the intermediate parameter space is not as well covered. Despite the importance of understanding galaxy evolution in the compact group environment, their complexity has caused progress to be extremely limited. Galaxy interactions in groups are more complicated to quantify than in isolated merger systems, and evolutionary scenarios must take into account both the interstellar and intergalactic media. Unlike clusters, however, groups tend to evolve on rapid dynamical time scales ( 400 Myr; Borthakur et al. 2010) and are typically not virialized.

To better understand galaxy evolution under these conditions we must study the impact and role of interstellar medium (ISM) processing amid continuous gravitational encounters. These encounters can generate and interact with an intragroup medium, and compact groups provide a window into this environment through the complex gravitational interactions of member galaxies. These systems can even reveal how gas processing affected galaxy build-up in the earlier universe. Present-day galaxy clusters are currently largely devoid of cold gas and active star formation but clearly built up substantial stellar mass sometime in the past. What happened to the cold gas - was it converted to stars, stripped, or heated? We can witness these processes in compact groups.

\section{$1.2 \quad$ Impact of Environment}

To understand how the compact group environment affects galaxy evolution, it is critical to look at galaxies in other environments. It is only through this comparison 
that we can determine how membership in a compact group influences galaxies. Comparison with other environments should include both higher galaxy number densities (e.g., clusters) and lower galaxy number densities (e.g., interacting pairs, isolated galaxies). How does star formation and gas content in compact groups differ from galaxies in other surroundings?

It is well known that morphology correlates with density (e.g., Dressler 1980)clusters have a significantly higher fraction of ellipticals than the field. This is also true in groups (Postman \& Geller 1984), and specifically compact groups (Lee et al. 2004). This is not surprising, as interactions and mergers are common in high-density regions. Star formation is also affected by environment - in clusters, star formation is seen to decrease as density increases, both inside and outside of clusters (e.g., Lewis et al. 2002; Kauffmann et al. 2004). In addition, small increases in galaxy density lead to older stellar populations (Blanton \& Berlind 2007; Blanton \& Moustakas 2009).

To differentiate the role of environment on galaxy evolution, I compare the properties of compact group galaxies with galaxies in different environments throughout this thesis. I endeavor to include surveys that target a range of galaxy densities. These comparison samples include galaxies from the LVL+SINGS surveys (Dale et al. 2007, 2009), the low-z survey of the NYU value-added galaxy catalog (VAGC; Blanton et al. 2005b,a; Adelman-McCarthy et al. 2008; Padmanabhan et al. 2008), interacting galaxies (Smith et al. 2007), and galaxies in the Coma cluster (Jenkins et al. 2007; Mahajan et al. 2010). Like the compact groups, I limit each of these samples to be at $z \leq 0.035$. These samples are described in more detail in the relevant chapters. 


\subsection{Compact Group Evolution}

Many studies have attempted to improve our understanding of the effect of the compact group environment by creating evolutionary sequences along which compact groups appear to mature. Verdes-Montenegro et al. (2001) and Johnson et al. (2007) have suggested evolutionary sequences based on the H I content of compact groups, while Konstantopoulos et al. (2010) also utilized the gas distribution. Coziol et al. (2004) suggest a sequence in which compact groups evolve from having low velocity dispersions and being rich in late-type galaxies that host star formation and/or an AGN to having high velocity dispersions and being dominated by quiescent early-type galaxies. Fuse \& Broming (2013) see a trend that groups with more luminous diffuse X-ray gas also have a higher luminosity of X-ray binaries. They interpret this as being caused by the same root - interactions simultaneously increase star formation (and thus the number of X-ray binaries) and heat the gas.

\subsection{Properties of Compact Groups}

The effect of the compact group environment is not yet totally understood - there are similarities with both clusters and the field. Like clusters, compact groups have a lower spiral fraction than the field (Hickson 1982). However, the interactions are more prolonged than in clusters, due to the low velocity dispersions in compact groups(Hickson et al. 1992). Despite these interactions, compact group galaxies tend to lie on the field Tully-Fisher relation (Mendes de Oliveira et al. 2003; Torres-Flores et al. 2010, 2013). Interpreting the impact of the compact group environment is complicated by the fact that the surrounding environment of compact groups is not consistent across (or even within) catalogs; some compact groups are isolated, while 
some are embedded in larger structures (Palumbo et al. 1995; Mendel et al. 2011).

Further complicating the definition of isolation is the presence of dwarf galaxies, as they are not necessarily taken into account in the definition of isolation. Dwarfs are common in CGs, and their presence affects the classification of evolutionary state (Konstantopoulos et al. 2013). However, star clusters are an excellent probe for determining the star-formation and merger history of a compact group (Konstantopoulos et al. 2010, 2012, 2013). Interactions and mergers in compact groups will affect the star formation and active galactic nuclei (AGN) (Coziol \& Plauchu-Frayn 2007). Compact group galaxies do not show signatures of strong AGN; in fact the frequency of AGN as a whole in compact groups is not high, and is in between that of clusters and the field (Martínez et al. 2010; Sohn et al. 2013). Even amongst the galaxies that do host an AGN, they are not impressive - compact groups have a deficiency of broad-line AGN (Martínez et al. 2008) and tend to host low-luminosity AGN (Coziol et al. 2004; Martínez et al. 2008, 2010) which will not dominate the emission from the host galaxy (Gallagher et al. 2008). This is interpreted as being caused by a lack of gas in compact group galaxies (Martínez et al. 2008, 2010; Sohn et al. 2013).

In the galaxy main-sequence plot, compact group galaxies have a slope of 0.51 using data from Tzanavaris et al. (2010). This is shallower than Daddi et al. (2007) with a slope of 0.9 as well as the slope of 0.67 for $\mathrm{M}^{10}<\mathrm{M}_{*}<\mathrm{M}^{11}$ over $\mathrm{z}=0.2-0.7$ quoted in Noeske et al. (2007), though they do note that there is a trend for slopes to flatten at higher $\mathrm{z}$.

\subsection{A Multi-wavelength Approach}

Given the complexity of these groups and the interplay between different physical properties, interpreting the physics requires a large, panchromatic dataset, as different 
physical properties are manifest at different wavelengths. For instance, the hot dust in these groups is apparent in the mid-infrared. Radio observations give us information on the cold gas, while X-rays reveal the hot, diffuse gas component of compact groups, as well as detecting AGN. To get a full picture of these compelx systems, we also need information on the stellar populations; optical colors can indicate whether light from a galaxy is dominated by young or old stars.

Optical colors have been key in probing galaxy populations throughout the uni-

verse; most galaxies fall in either the "blue cloud" of galaxies with young stars or the "red sequence" of galaxies either dominated by older stellar populations or hosting obscured star formation. Between the blue, active galaxies and the red and dead galaxies lies the underpopulated "green valley" (e.g. Hogg et al. 2004). Thus optical colors of galaxies can be indicative of the star formation history, and may be vital to understanding how gas processing has affected the stellar populations. It is only by combining optical and mid-IR data that we can get a full picture of the star formation and stellar populations of galaxies.

\subsection{Star Formation in Compact Groups}

Star formation in compact groups is intriguing - some groups show evidence of moderate or even intense star formation (Iglesias-Páramo \& Vílchez 1999; Gallagher et al. 2010). However, IRAS observations of compact groups indicate normal levels of thermal infrared emission, suggesting that the star formation rates (SFRs) in these groups are not generally enhanced (Allam et al. 1995; Verdes-Montenegro et al. 1998). In fact, de la Rosa et al. (2007) studied the stellar populations of elliptical galaxies in compact groups and find evidence of a mechanism that quenches star formation. This is again similar to the outer regions of clusters, which exhibit suppression of SFRs out 
to three virial radii (Lewis et al. 2002). Gas processing is also different in cluster cores and compact groups, where ram-pressure stripping does not appear to dominate gas processing (Rasmussen et al. 2008). In addition, Cortese et al. (2006) have studied a compact group falling into a cluster, and found that the galaxies have undergone preprocessing due to the compact group environment. Despite their high densities, it appears as though the compact group environment has more in common with the outer regions of clusters than with cluster cores.

Nor are compact group galaxies similar to field galaxies - the fraction of ellipticals in compact groups is higher than that of the field (Lee et al. 2004; Deng et al. 2008; Coenda et al. 2012). Many authors (e.g., Proctor et al. 2004; Mendes de Oliveira et al. 2005; de la Rosa et al. 2007; Plauchu-Frayn et al. 2012) have found that, on average, ellipticals in compact groups are older than in the field, more like cluster galaxies. Amongst spirals, Plauchu-Frayn et al. (2012) find that early-type spirals have a lower specific star formation rate (SSFR) than field galaxies, while late-type spirals have a higher SSFR. Martinez-Badenes et al. (2012) find that galaxies with higher gas deficiencies $\left(M_{H_{I}}\right.$ or $\left.M_{H_{2}}\right)$ have lower SSFR than isolated galaxies, but that their star formation efficiency is similar. These results all point to the importance of the gas in compact groups, and how it is affected by the interactions in compact groups.

\subsection{Current Knowledge of the Cold Gas}

Understanding galaxy evolution in the dense compact group environment requires not only characterizing each galaxy but also the overall group properties and dynamics. Gas in these systems is key - it provides fuel for star formation, responds to shocking and dissipation, traces dynamical interactions, provides information about the phase of the interstellar medium (e.g. hot, warm, cold), and influences the resulting galaxy. 

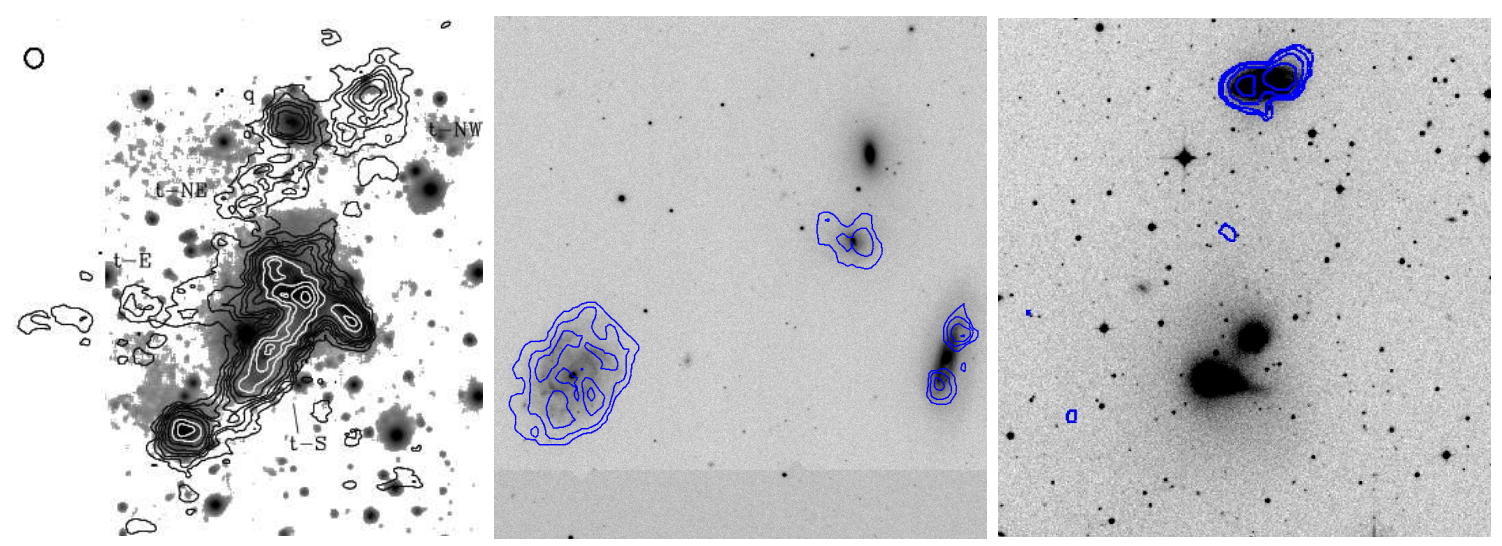

Fig. 1.1. - Existing case studies show a wide variety of H I distributions (contours): left - spread throughout the group into the intra-group medium in HCG 31 (VerdesMontenegro et al. 2005); center - contained within the member galaxies in HCG 7 (A. Heiderman, priv. comm.); right-detected only in one galaxy in HCG 90 (Rupen et al. 2001).

Single-dish studies of molecular gas have revealed the complexity of compact groups - Boselli et al. (1996) and Leon et al. (1998) find an excess of CO emission compared to normal galaxies, while Verdes-Montenegro et al. (1998) find that $20 \%$ of galaxies in compact groups show a deficit and the remainder have typical CO emission.

Complexity is also seen in the atomic gas properties: as a class, compact groups are H I-deficient (Verdes-Montenegro et al. 2001); however, they exhibit a wide range of H I content - from HCG 33, which contains more H I than predicted, to HCG 54, which barely contains any H I (Verdes-Montenegro et al. 2001). Not only can the diversity of $\mathrm{H}$ I deficiences provide instrumental clues about galaxy evolution in dense environments, but it is also manifest in the distribution of $\mathrm{H} \mathrm{I}$ in compact groups (Fig. 1.1). This suggests that gas is removed from galaxies during group evolution. This is supported by the fact that like clusters, compact groups that are more deficient in H I have a higher rate of detection in X-ray (Giovanelli \& Haynes 1985; VerdesMontenegro et al. 2001), though Desjardins et al. (2013) finds that X-ray emission in compact groups tends to be associated with individual galaxies, rather than the 
overall group. Thus, these systems allow us to witness the physical mechanisms that remove or deplete gas through galaxy interactions (e.g., stripping, star formation,

heating). Gas processing can follow several different paths. Stripping - either tidal or ram-pressure (Gunn \& Gott 1972) - can remove gas from galaxies which can then be heated into an X-ray halo. However, Desjardins et al. (submitted) found that the hot gas in compact groups is usually associated with individual galaxies, thus rampressure stripping is unlikely to play a large role in compact groups. In the cluster environment, gas and stars can also be removed by galaxy harassment (Moore et al. 1998), which would also build up an intra-group medium, potentially heated to Xray temperatures. Gas can also be converted into stars within individual galaxies, as some compact groups do show an enhancement of star formation, or could be blown out from individual galaxies when young stars explode as a supernova. This redistribution of material in compact groups results in a range of astrophysically important processes, making compact groups an essential laboratory for studying galaxy evolution. A comprehensive study of the different states of gas in a large sample of compact groups - atomic, molecular, and hot ionized - has never been done.

\subsection{Compact Group Catalogs}

In compiling the samples used in this work, I searched many compact group catalogs in an effort to create a statistically significant dataset. In addition to the criteria used to identify a compact group by each individual study, I implemented my own criteria: a significant portion of the groups had to be at a redshift of $z \leq 0.035$, so that we could properly interpret their colors, and they had to have an angular extent $\theta<8^{\prime}$ so as to be easily observable with facilities such as Spitzer Space Telescope. 


\subsubsection{Hickson Compact Groups}

The most well-known catalog of compact groups is the Hickson Compact Group catalog (HCGs; Hickson 1982). Groups from this catalog, compiled through visual inspection of photometric plates from the Palomar Sky Survey, have been extensively studied. In compiling this catalog, Hickson set three criteria: population - the groups must have at least four members whose magnitudes are within three magnitudes of the brightest galaxy $(N \geq 4)$; isolation - the nearest galaxy of comparable brightness must be at an angular separation no less than three times the size of group $\left(\theta_{N} \geq 3 \theta_{G}\right)$; and compactness - the surface brightness of the group must be high $\left(\bar{\mu}_{G}<26.0\right)$. Subsequent study of these groups by Hickson et al. (1992) utilized redshifts to determine the dynamical properties of these groups, this work revealed that eight groups are not true associations, and that some groups actually only have three members. The average redshift of the 92 true HCGs is $z=0.034$.

\subsubsection{Redshift Survey Compact Groups}

A relatively under-studied catalog of compact groups is the Redshift Survey Compact Group catalog (RSCGs; Barton et al. 1996). This catalog was compiled from magnitude-limited CfA redshift survey data using a friends-of-friends algorithm. The criteria used to locate compact groups and determine their members were chosen to identify compact groups similar to the HCGs. Members of compact groups were identified as linked sets of neighbors based on projected separation of $D \leq 50 \mathrm{kpc}$

and radial velocity difference of $V \leq 1000 \mathrm{~km} \mathrm{~s}^{-1}$. As these criteria were chosen to select HCG-like compact groups, some of the groups in this catalog are in fact HCGs. However, one notable difference in their selection criteria is the lack of an isolation requirement; this means that some of the RSCGs are embedded in larger structures. 
The average redshift of the 89 RSCGs is $z=0.014$.

\subsubsection{Other Catalogs}

There are other catalogs of compact groups, many of which have been compiled in a manner similar to either the HCGs or RSCGs. However, none satisfy the criteria to such an extent as the HCGs and RSCGs. Some of these catalogs are the Shakhbazyan compact groups (Shakhbazyan 1973), the Updated Zwicky Catalog (UZC) compact groups (Focardi \& Kelm 2002), the Southern compact groups (Prandoni et al. 1994), the Las Campanas compact groups (Allam \& Tucker 2000), and compact groups from the SDSS commissioning data (Lee et al. 2004). Properties of these catalogs, along with the HCGs and RSCGs, are listed in Table 1.1.

\subsection{Organization of this Thesis}

This thesis is comprised of three published papers and one paper in preparation, described briefly below.

Chapters 2 (Walker et al. 2010) and 3 (Walker et al. 2012) investigate the mid-IR properties of compact group galaxies. I place compact group galaxies in context with galaxies in other environments, and find that the compact group environment must be similar to that of the Coma Infall Region.

Chapter 4 (Walker et al. 2013) reports the results of an optical study of compact group galaxies. I compare optical and mid-IR colors and find that the mid-IR transition galaxies fall on the optical red sequence. Furthurmore, a study of LVL+SINGS galaxies reveals that the dearth of mid-IR transition galaxies in compact groups may indicate that compact groups are inhospitable to low-mass, moderately star-forming galaxies. 
Chapter 5 highlights a survey of neutral hydrogen (H I) in RSCGs. I compute group H I masses and deficiencies, and compare these with galaxy properties.

Finally, chapter 6 summarizes my results and details work yet to be done in the study of compact groups. 
Table 1.1. Catalogs of Compact Groups

\begin{tabular}{c|ccc}
\hline \hline CG Catalog & $N_{\text {groups }}$ & $\langle z\rangle$ & $\begin{array}{c}\langle\theta\rangle^{\dagger} \\
\left.{ }^{\prime}\right]\end{array}$ \\
\hline Hickson & 92 & 0.034 & 3.7 \\
Redshift Survey & 89 & 0.014 & 10.7 \\
Shakhbazyan & 377 & $\ldots$ & 2.2 \\
Southern & 59 & $\ldots$ & 4.0 \\
UZC & 291 & 0.017 & $\ldots$ \\
Las Campanas & 76 & 0.079 & 1.94 \\
SDSS & 177 & 0.135 & 0.52 \\
\hline
\end{tabular}

Note. - †For all catalogs except the Shakhbazian catalog this is the diameter of the group. The Shakhbazian catalog only lists "size" - it is unclear whether this is radius or diameter. 
Chapter 2

Mid-Infrared Evidence for

Accelerated Evolution in Compact Group Galaxies 


\section{Abstract}

Compact galaxy groups are at the extremes of the group environment, with high number densities and low velocity dispersions that likely affect member galaxy evolution. To explore the impact of this environment in detail, we examine the distribution in the mid-IR $3.6-8.0 \mu \mathrm{m}$ colorspace of 42 galaxies from 12 Hickson compact groups in comparison with several control samples, including the LVL+SINGS galaxies, interacting galaxies, and galaxies from the Coma Cluster. We find that the HCG galaxies are strongly bimodal, with statistically significant evidence for a gap in their distribution. In contrast, none of the other samples show such a marked gap, and only galaxies in the Coma infall region have a distribution that is statistically consistent with the HCGs in this parameter space. To further investigate the cause of the HCG gap, we compare the galaxy morphologies of the HCG and LVL+SINGS galaxies, and also probe the SSFR of the HCG galaxies. While galaxy morphology in HCG galaxies is strongly linked to position with mid-IR colorspace, the more fundamental property appears to be the SSFR, or star formation rate normalized by stellar mass. We conclude that the unusual mid-IR color distribution of HCG galaxies is a direct product of their environment, which is most similar to that of the Coma infall region. In both cases, galaxy densities are high, but gas has not been fully processed or stripped. We speculate that the compact group environment fosters accelerated evolution of galaxies from star-forming and neutral gas-rich to quiescent and neutral gas-poor, leaving few members in the mid-IR gap at any time.

This was my first project in graduate school. I used fluxes that my advisor, Kelsey Johnson, had previously measured for the compact group galaxies as well as fluxes from the literature for our comparison samples. I performed the KolmogorovSmirnov tests, made mid-IR color-magnitude diagrams, and compared colors with 
morphologies. I investigated the possibility that the gap galaxies were post-starburst by gathering spectra and measuring the equivalent width of the $\mathrm{H} \alpha$ line. I also discovered that the IRAC colorspace gap corresponds to the gap in SSFR. Finally, I wrote the chapter.

\section{$2.1 \quad$ Introduction}

A challenge of studying HCGs is moving from a morphology-based to a quantitatively based description of the star-formation and evolution within the extreme populationdensity environment. Smith et al. (2007) find that the mass-normalized SFR in interacting galaxies are approximately twice that in normal spirals, lending support to the idea that interactions induce star formation. However, de la Rosa et al. (2007) and others find evidence of a mechanism that quenches the star formation in compact groups. Specifically, they find that the stellar populations in elliptical galaxies in HCGs are more metal-poor and older than their counterparts in the field. Thus, although compact groups might be thought to be an ideal site for merger-induced star formation, this does not always seem to be the case.

In order to assess the impact of the compact group environment on star formation, Johnson et al. (2007) looked at the Spitzer IRAC (3.6-8.0 $\mu \mathrm{m})$ colorspace distribution of HCGs and found that the mid-infrared (mid-IR) colors of galaxies in H I gasrich HCGs are dominated by star formation, while the mid-IR colors of galaxies in H I gas-poor HCGs are dominated primarily by stellar photospheric emission, or are mid-IR-passive. From this, they infer an evolutionary sequence in which the gas in H I-rich groups is consumed, expelled, or ionized. In a complementary study of loose groups at higher redshift ( $\mathrm{z} \sim 0.4$ ), Wilman et al. (2008) see a bimodality in $k$ -

corrected $[f(8.0) / f(3.6)]$ colors, and a deficit of infrared activity when compared to 
field galaxies, similar to local H I-poor compact groups. They note that the fraction of infrared excess galaxies, $f(I R E)$ decreases with galaxy stellar mass $M_{*}$, but within their group sample they see a deficit in $f(I R E)$ at all masses, and state that this trend can be explained if suppression of $M_{*}>10^{11} M_{\odot}$ galaxies occurs primarily in the group environment.

In Spitzer IRAC color-color plots of HCG galaxies, Johnson et al. (2007) noted a "gap" in the distribution of galaxies between those dominated by stellar light and galaxies that are actively star-forming. This gap did not appear to be present in the initial comparison sample from the Spitzer First Look Survey (FLS). This comparison led them to speculate that the gap may be due to rapid evolution caused by the unique dynamical influences present in HCGs. However, the range in redshift of the FLS galaxies, which shifts polycyclic aromatic hydrocarbon (PAH) features in and out of the observing bands, renders the plot ambiguous. Nonetheless, Gallagher et al. (2008), using a related mid-IR diagnostic, confirmed the discrepancy by comparing HCG galaxies to the local SINGs sample (Dale et al. 2007), which like the FLS sample exhibits no gap in mid-IR color space. Tzanavaris et al. (2010) determined SFRs and specific SFRs (SSFRs) for this same HCG sample using Swift UV and Spitzer $24 \mu \mathrm{m}$ data and found a gap in SSFRs between $3.2 \times 10^{-11} \mathrm{yr}^{-1}$ and $1.2 \times 10^{-10} \mathrm{yr}^{-1}$. This gap did not exist in their comparison sample comprised of SINGS non-interacting and isolated galaxies. The fact that the HCG sample is discrepant with both the FLS and SINGS samples led them to conclude that the local environment in HCGs strongly influences the member galaxies.

Galaxies in compact groups likely evolve differently than galaxies in other environments. Galaxies in clusters also experience frequent interactions, but the interaction timescale is shorter due to the large velocity dispersions, and they reside in a smoother 
gravitational potential, causing less torquing on the gas within galaxies. On the other hand, galaxies in loose groups experience less frequent interactions on average than those in compact groups. Giant field galaxies experience fewer major interactions, and frequently dominate their local gravitational potential. Comparing galaxies in high density environments (e.g. clusters, compact groups) with galaxies in medium density environments (e.g. interacting pairs of galaxies) and low density environments (e.g. individual field galaxies) may allow greater understanding of how galaxy evolution is affected by the local density.

In an effort to learn more about the nature of the gap found by Johnson et al. (2007), we have examined the mid-IR colors of other samples of galaxies in a variety of environments. The previous comparison samples, FLS (Johnson et al. 2007) and SINGS (Gallagher et al. 2008; Tzanavaris et al. 2010) were both limited in scope and are known to suffer from issues with the samples that could have led to their discrepancy with the HCG sample. The goal of this paper is to expand the comparisons to a range of other samples with different properties in order to better assess the influence of environment on mid-IR colorspace distribution. We use the Kolmogorov-Smirnov test to compare the mid-IR colors of the galaxies in these samples with those of the HCG galaxies to investigate to what extent the gap seen by Johnson et al. (2007) is ubiquitous, or unique to the compact group environment.

\subsection{Data/Samples}

\subsubsection{HCG Galaxies}

The HCG dataset, taken from Johnson et al. (2007), comprises 42 galaxies from 12 groups. The groups contain varying amounts of H I, and span the three classifica- 


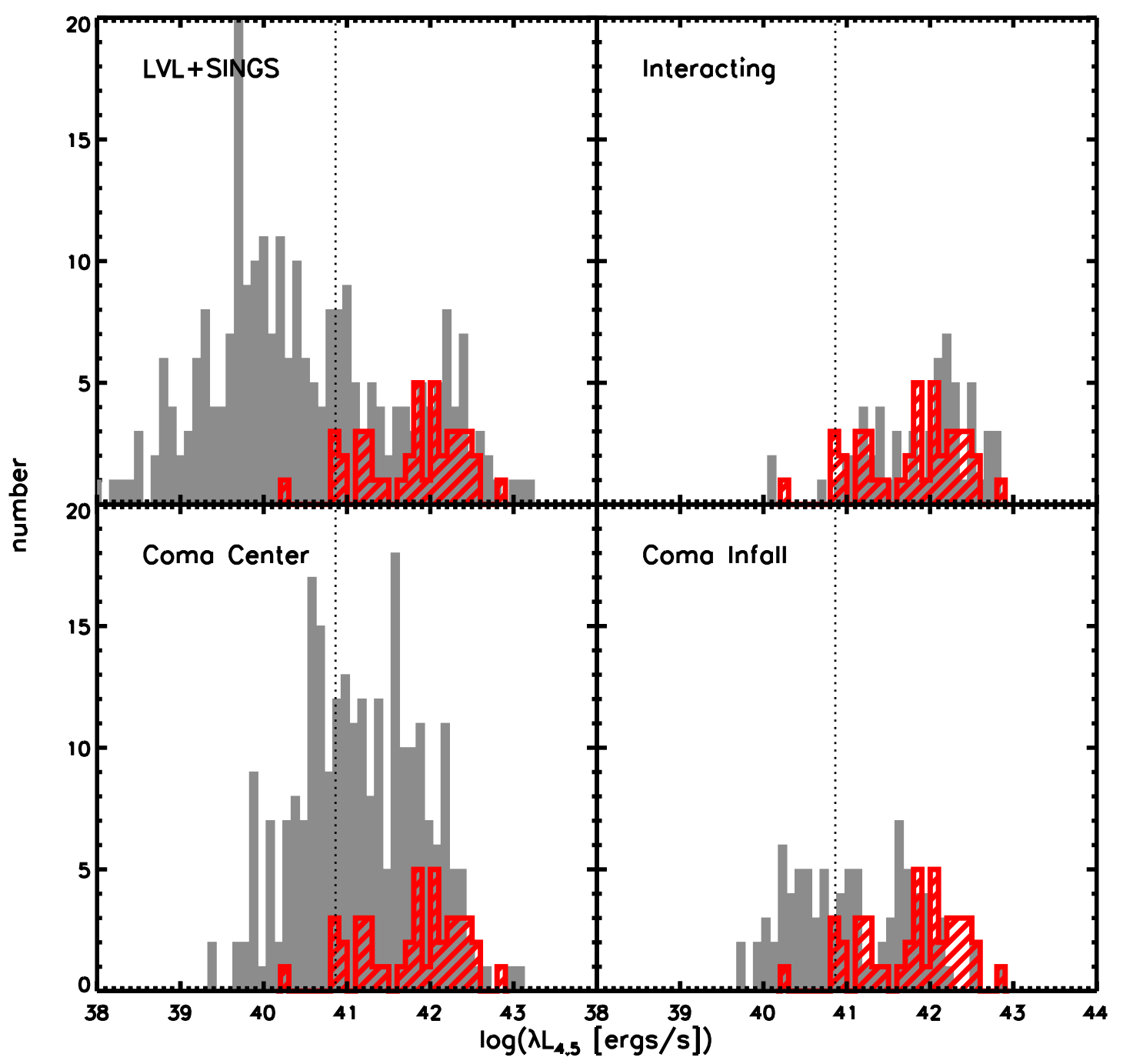

Fig. 2.1. - Histograms of $\lambda L_{4.5}$ for each sample, with the HCG galaxies overlaid with the red striped histogram. The histograms yield similar results regardless of whether $\lambda L_{3.6}$ or $\lambda L_{4.5}$ is used. The dotted vertical line indicates the minimum luminosity for comparison and statistical analysis between samples. 


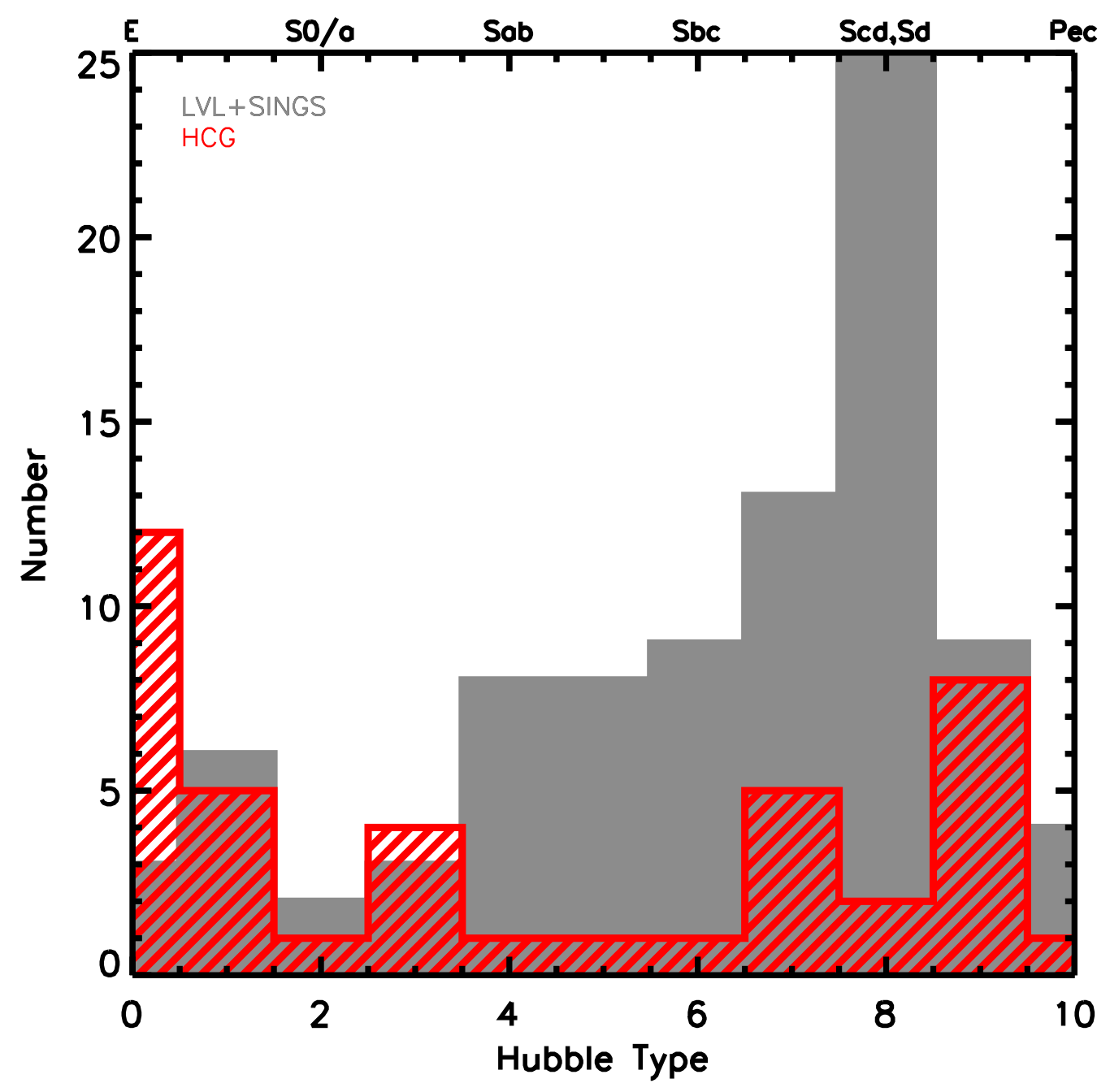

Fig. 2.2. - Histograms of Hubble type (following Haynes \& Giovanelli 1984) for the HCG galaxies (striped) and LVL+SINGS galaxies (solid). 
tion categories discussed in Johnson et al. (2007). The most H I gas-rich groups with $\log \left(M_{\mathrm{HI}}\right) / \log \left(M_{\mathrm{dyn}}\right) \geq 0.9$ are classified as type I, the H I gas-poor groups with $\log \left(M_{\mathrm{HI}}\right) / \log \left(M_{\mathrm{dyn}}\right)<0.8$ as type III, while type II contains the intermediate groups. It is also important to note that these categories reflect the group $\mathrm{H}$, which is not necessarily simply the sum of the H I content of individual galaxies as this classification also includes gas in the IGM. Table 2.1 gives background data on these groups. NED has been searched for known AGN, and four Seyfert IIs were identified: HCG 16b, 16d, 61a, and 90a. However, we expect emission from the AGN in these cases should be minimal compared to the integrated light of the galaxy, as found by Gallagher et al. (2008).

One question brought up in discussions of compact groups (CGs), especially HCGs, is the effect of selection biases. Mamon (1994) claims that the properties of CGs are a function of the algorithm used to find them. Ribeiro et al. (1998) discusses Hickson's original selection criteria and how they influence the catalog of HCGs, such as ignoring the faint end of the luminosity function, selecting only groups with "significant surface density enhancement over the field," and appearing to be well isolated, taking them out of context with their surroundings. Since Hickson's original catalog, several other catalogs of CGs have been published, some based on sky position and photometry like Hickson's, some utilizing redshift data. With the flood of data from SDSS, several catalogs of CGs have been generated, allowing selection effects to be qualitatively studied. Lee et al. (2004) selected CGs from the SDSS commissioning data using criteria slightly modified from Hickson's; they instituted an upper limit on number of group members, changed the isolation criteria to greater than three angular diameters, rather than greater than or equal to, and required higher surface brightness. They compared their catalog with six existing 
catalogs of CGs, including the HCGs, which they acknowledge as "the benchmark for all CG catalogs." For each CG catalog, they looked at the mean and median of group members, surface brightness, redshift, angular diameter, and linear diameter, and the HCGs tend to fall in the middle of each distribution (Table 3 in Lee et al. 2004). Thus, it appears that any selection biases that affect the HCGs are either more strongly present in other catalogs, or inherent properties of CGs as a class. In the latter case, this can be interpreted as reflecting the role of the CG environment on the evolution of the constituent galaxies.

\subsubsection{Comparison Samples}

The LVL data, discussed in Dale et al. (2009), consists of 258 galaxies within 11 Mpc. Galaxies with undefined flux values or only upper limits on the photometry were excluded, leaving 211 galaxies in the sample. The SINGS data, as presented and described in Dale et al. (2007), consist of 71 galaxies. Four of the galaxies in the original dataset (M81 Dwarf A, NGC 3034, Holmberg IX, and DDO 154) were excluded because only upper or lower limits on the photometry were provided. It is important to note that the SINGS sample was chosen to be diverse, which will affect its distribution in colorspace. In addition, we combined the LVL and SINGS galaxies to create a control sample, referred to as LVL+SINGS. The 35 galaxies from the Spitzer Spirals, Bridges, and Tails Interacting Galaxy Survey (hereafter referred to as the interacting sample) are comprised of otherwise relatively isolated binary galaxy systems, whose members are tidally disturbed (Smith et al. 2007). This sample was biased towards galaxies with prominent signs of interaction, thereby selecting against

elliptical galaxies. The Coma sample, discussed in Jenkins et al. (2007), is comprised of galaxies from two fields. The first field is located in the center of the cluster, where 
Table 2.1. Properties of HCG Sample

\begin{tabular}{cccccc}
\hline \hline HCG & $\begin{array}{c}\text { R.A. } \\
(\mathrm{J} 2000)\end{array}$ & $\begin{array}{c}\text { Dec } \\
(\mathrm{J} 2000)\end{array}$ & $\begin{array}{c}\mathrm{D}^{\mathrm{a}} \\
(\mathrm{Mpc})\end{array}$ & $\begin{array}{c}\log M_{\mathrm{H} \mathrm{I}} \mathrm{b} \\
\left(M_{\odot}\right)\end{array}$ & Group Type $^{\mathrm{c}}$ \\
\hline 02 & $0^{\mathrm{h}} 31^{\mathrm{m}} 30^{\mathrm{s}} .0$ & $+8^{\circ} 25^{\prime} 52^{\prime \prime}$ & 136.6 & 10.33 & $\mathrm{I}$ \\
07 & $0^{\mathrm{h}} 39^{\mathrm{m}} 23^{\mathrm{s}} .9$ & $+0^{\circ} 52^{\prime} 41^{\prime \prime}$ & 56.4 & 9.68 & II \\
16 & $02^{\mathrm{h}} 09^{\mathrm{m}} 31^{\mathrm{s}} .3$ & $-10^{\circ} 09^{\prime} 31^{\prime \prime}$ & 52.9 & $>10.42$ & $\mathrm{I}$ \\
19 & $02^{\mathrm{h}} 42^{\mathrm{m}} 45^{\mathrm{s}} .1$ & $-12^{\circ} 24^{\prime} 43^{\prime \prime}$ & 57.3 & 9.31 & II \\
22 & $03^{\mathrm{h}} 03^{\mathrm{m}} 31^{\mathrm{s}} .3$ & $-15^{\circ} 40^{\prime} 32^{\prime \prime}$ & 36.1 & 9.13 & II \\
31 & $05^{\mathrm{h}} 01^{\mathrm{m}} 38^{\mathrm{s}} .3$ & $-04^{\circ} 15^{\prime} 25^{\prime \prime}$ & 54.5 & 10.35 & I \\
42 & $10^{\mathrm{h}} 00^{\mathrm{m}} 21^{\mathrm{s}} .8$ & $-19^{\circ} 38^{\prime} 57^{\prime \prime}$ & 53.3 & 9.40 & III \\
48 & $10^{\mathrm{h}} 37^{\mathrm{m}} 45^{\mathrm{s}} .6$ & $-27^{\circ} 04^{\prime} 50^{\prime \prime}$ & 37.7 & 8.52 & III \\
59 & $11^{\mathrm{h}} 48^{\mathrm{m}} 26^{\mathrm{s}} .6$ & $+12^{\circ} 42^{\prime} 40^{\prime \prime}$ & 54.1 & 9.49 & II \\
61 & $12^{\mathrm{h}} 12^{\mathrm{m}} 24.9$ & $+29^{\circ} 11^{\prime} 21^{\prime \prime}$ & 52.1 & 9.96 & I \\
62 & $12^{\mathrm{h}} 53^{\mathrm{m}} 08^{\mathrm{s}} .1$ & $-09^{\circ} 13^{\prime} 27^{\prime \prime}$ & 54.9 & $<9.06$ & III \\
90 & $22^{\mathrm{h}} 02^{\mathrm{m}} 06^{\mathrm{s}} .0$ & $-31^{\circ} 55^{\prime} 48^{\prime \prime}$ & 32.3 & 8.66 & III \\
\hline
\end{tabular}

${ }^{a}$ Group distance taken from NED. The cosmology used was $H_{0}=$ $73 \mathrm{~km} \mathrm{~s}^{-1} \mathrm{Mpc}^{-1}, \Omega_{\text {matter }}=0.27$, and $\Omega_{\text {vacuum }}=0.73$.

${ }^{\mathrm{b}} \mathrm{H}$ I masses from Verdes-Montenegro et al. (2001), with the exception of HCG 90, provided by J. Hibbard 2006, private communication.

${ }^{\mathrm{c}}$ Group type discussed in $\S 2.2 .1$. Type $\mathrm{I}=\mathrm{H}$ I-rich, Type III $=\mathrm{H}$ Ipoor. 
the galaxy density is very high. The second field is the infall region, located near 0.4 virial radii at the secondary X-ray peak, where the galaxy density is still higher than field density (Jenkins et al. 2007). The 104 galaxies in the $0.555 \mathrm{deg}^{2}$ of the infall region yield a surface density of $187 \mathrm{gal} / \mathrm{deg}^{2}$. If we assume a non-physical flat density profile, we expect to find 137 galaxies in the $0.733 \mathrm{deg}^{2}$ field of Coma center, but the density in the center could easily be twice as high as that in the Coma Infall region, yielding 274 galaxies in this region. Thus, there may be contamination in the center sample from cluster galaxies in front of and behind the actual center. Two galaxies from the center of the Coma cluster have been removed from the sample due to uncertain apertures, caused by proximity to one of the central elliptical galaxies. Our HCG sample has only one galaxy with luminosity below $\log \left(L_{4.5}[\mathrm{erg} / \mathrm{s}]\right)=40.9$. With Spitzer, we mapped the entire extent of each group to a 4.5 micron sensitivity better than this limit (Johnson et al. 2007), thus our HCG database should be complete to this level. To be conservative, to compare the HCG galaxies to similar galaxy populations from the other samples we only consider galaxies with higher luminosities. The luminosity distributions of the samples are shown in Figure 2.1, with a vertical dotted line indicating the minimum luminosity required for inclusion. Figure 2.2 shows the distribution of Hubble types in our HCG sample compared with the LVL+SINGS sample. As can be seen in this plot, the LVL+SINGS sample is deficient in Sa galaxies, with respect to other Hubble types. The LVL+SINGS sample is dominated by spirals later than Sa, while the HCG sample contains galaxies across the distribution. 


\subsection{Mid-Infrared Colorspace}

\subsubsection{HCG Galaxies}

The IR color-color plot for the HCG galaxies is shown in Figure 2.3. The galaxies are separated into three types based on the fractional H I mass of the groups they belong to - the indigo plus signs represent type I (H I gas-rich), the red triangles indicate type II, and the blue squares correspond to type III (H I gas-poor). Galaxies in the lower-left portion of the plot have IR spectral energy distributions (SEDs) consistent with being dominated by stellar light. H I gas-poor groups fall in this region of the diagram due to the higher percentage of E/S0 galaxies. Galaxies with ongoing star formation will tend to fall in the upper right with red mid-IR colors indicative of interstellar polycyclic aromatic hydrocarbon (PAH) emission and thermal emission from warm/hot dust. The majority of the galaxies in the active region of the plot come from both type I (H I-rich) and type II groups, though there is a trend that galaxies from type II groups fall in the blue part of the active region, while the galaxies from type I groups show a larger range of colors throughout the region of the plot indicating activity. This trend can also be attributed to the percentage of E/S0 galaxies, as the type I groups contain very few E/S0 galaxies, while the type II groups have a varying percentage of $\mathrm{E} / \mathrm{S} 0$ galaxies.

As Johnson et al. (2007) noted, it is apparent in Figure 2.3 that the region between the galaxies dominated by stellar emission and galaxies with active star formation contains relatively few galaxies. In the following sections, we will explore the hypothesis that this gap in the mid-IR colors of HCG galaxies is due to rapid evolution through the stage during which galaxies have intermediate mid-IR colors, or in other words, the HCG environment is biased against galaxies with very modest amounts of star 


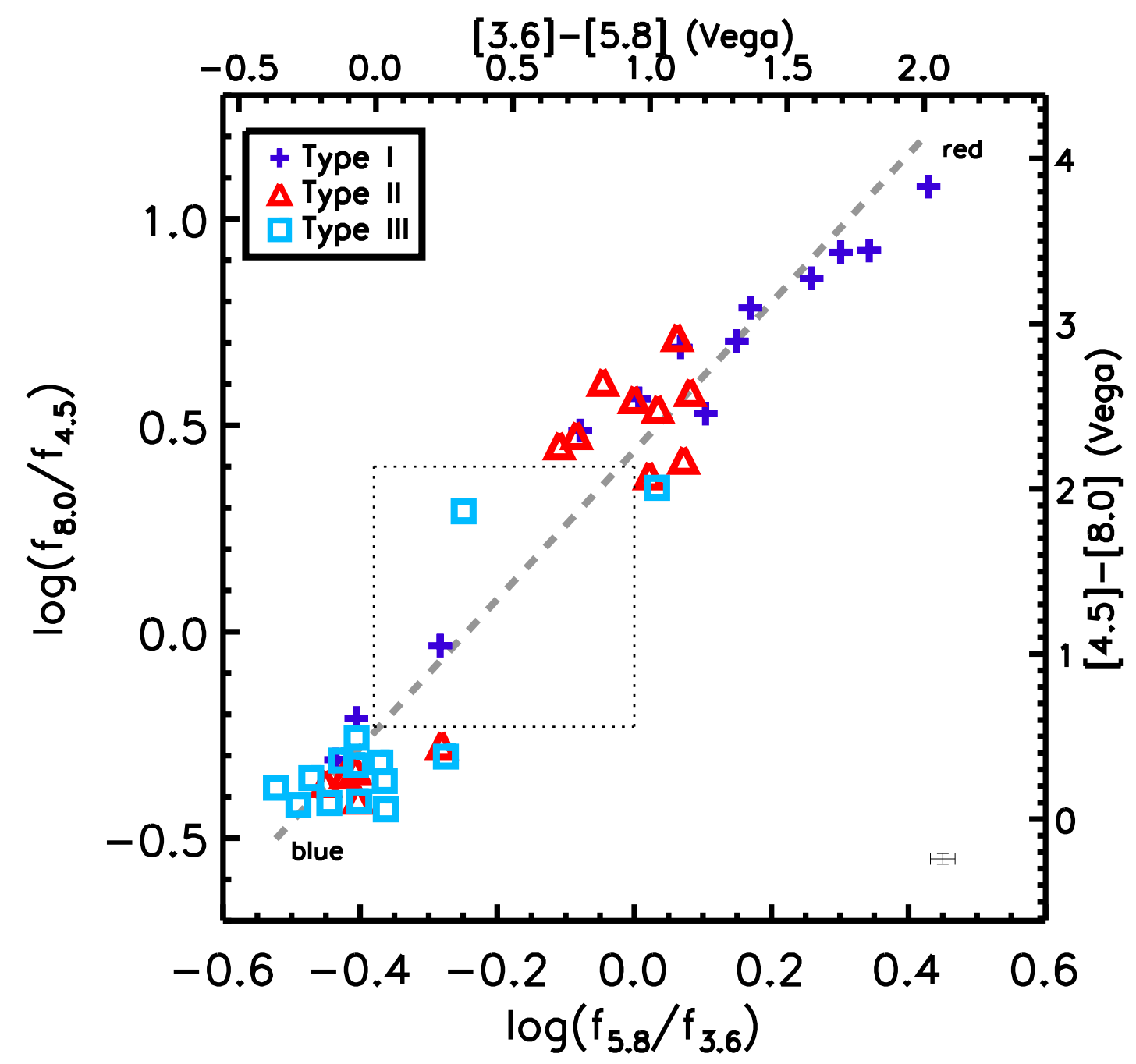

Fig. 2.3.- Color-color plot of HCG galaxies. The indigo plus signs are galaxies from type I (H I gas-rich) groups, the red triangles correspond to galaxies from type II groups, and the blue squares represent galaxies from type III (H I gas-poor) groups. The error bars in the lower right of the plot indicate typical errors for the sample. The lower-left region of the plot contains galaxies whose light is dominated by stellar photospheric emission, while actively star-forming galaxies reside in the upper right. Between these is the "gap" noted by Johnson et al. (2007), indicated by the dotted box. The dashed line is the linear fit to the data, used for coordinate rotation in $\S 2.4$. 
formation.

\subsubsection{Comparison Samples}

The LVL+SINGS galaxies span the same region in mid-IR colorspace as the HCG galaxies, as shown on the top left in Figure 2.4. Given that the LVL is a volumelimited survey and SINGS galaxies were chosen to represent a range of physical properties and environments, it is not surprising that they are distributed relatively uniformly in colorspace rather than clustered in one region. Figure 2.4 does not show any evidence of a gap in colorspace for the LVL+SINGS galaxies. A plot made with the culled SINGS sample of Smith et al. (2007) (not shown) is similar, and also does not show a gap. The culled sample was created by removing galaxies with nearby companions from the SINGS sample.

The sample of interacting galaxies (top right plot of Figure 2.4) forms a very tight relationship in the star-forming region of colorspace, which is consistent with the fact that interactions frequently trigger star formation. There is only one point whose colors are consistent with being dominated by stellar populations, with little, if any, PAH emission and thermal emission from warm dust. The selection against elliptical galaxies mentioned in $§ 2.2 .2$ also likely contributes to the lack of points in the blue region of IRAC colorspace.

The majority of the galaxies in the center of the Coma cluster lie in the region of mid-IR colorspace corresponding to stellar colors, with a smaller number of the galaxies scattered throughout the redward side of the plot. This is consistent with the fact that galaxies in the cores of clusters tend to contain little to no H I and star formation. Unlike the other samples we consider, the central Coma galaxies do not follow a tight relationship in colorspace but rather have a much more scattered 


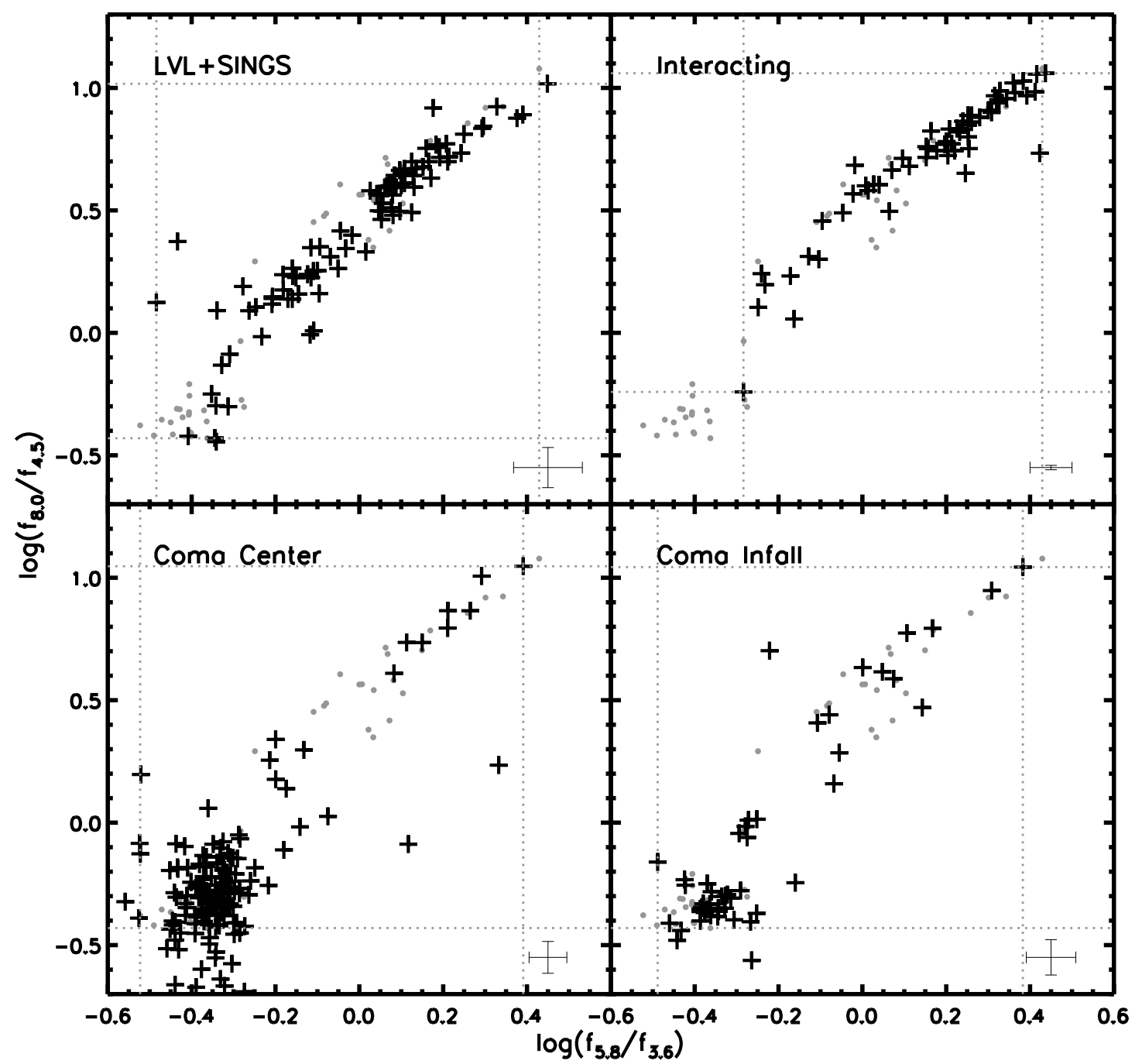

Fig. 2.4. - Color-color plot of the comparison samples. The grey dots are the HCG galaxies, and the error bars in the bottom right indicate typical errors for each comparison sample. The dotted lines indicate the cropping values, discussed in $\S 2.4$. The LVL+SINGS sample is distributed rather uniformly in colorspace. The interacting sample is located primarily in the active (upper right) region of the plot. The two Coma samples have a significant population in the stellar colors (lower left) region of colorspace; central Coma galaxies show no sign of a gap, while the infall Coma sample shows an underdensity of points in the same location as the HCG gap. 
distribution. There are two galaxies with unusual colors: red $\log \left(f_{5.8} / f_{3.6}\right)$ and blue

$\log \left(f_{8.0} / f_{4.5}\right)$. These two galaxies do not appear anomalous in the IRAC data, and we were unable to find anything in the literature which might explain their odd location in IRAC colorspace. However, both of these galaxies are in very crowded fields, and that may be the cause of their odd colors. The central Coma galaxies are rather smoothly distributed, with no sign of a gap in their distribution.

Despite being from the same galaxy cluster, the distribution in colorspace of galaxies in the infall region of the Coma cluster is less concentrated in the region of stellar colors than the center of the Coma cluster. There are galaxies whose light is dominated by stellar emission, but the star-forming region for the infall sample is relatively more populated than the central Coma region. The color-color plot of galaxies in the infall region of the Coma cluster reveals an underdensity of points in the same location as the gap we see in the HCG galaxies. While it does not seem to be as pronounced, it may still carry some significance.

\subsection{Statistical Tests}

In order to assess whether the gap in the distribution of HCG galaxies seen in Figure 2.3 is significant, we perform a statistical analysis using the Kolmogorov-Smirnov (KS) test. The IDL routines ksone and kstwo were used to perform the KS test, which first required rotating the data. The single-distribution version of the test allows comparison of each sample to a model of a uniform distribution, and determines whether it is an accurate description of the data. The two-distribution version of the test compared two samples to determine whether they could have been drawn from the same distribution. Notably, it is only possible to conclusively reject the null hypothesis using this method; the KS test cannot confirm the hypothesis. 
We performed the two-distribution test in two ways: 1) comparing only the region in colorspace that is populated by both the HCG sample and the comparison sample (indicated by the dotted lines in Figure 2.4), from which we cannot draw any conclusions about the distributions outside of this range; and 2) comparing the entire region of colorspace populated by either the HCG sample or the comparison sample. We fit a line to the HCG distribution in colorspace, which became the new $\mathrm{x}$-axis in rotated colorspace, $\Delta C_{M I R}$.

It is important to note that a "uniform" distribution does not necessarily mean a "normal" or "expected" distribution. A uniform distribution of galaxies in colorspace would be a sample whose galaxies fall evenly along a trend in colorspace. However, a normal distribution for old elliptical galaxies would show the galaxies preferentially in a clump in the bottom-left, stellar colors region. In a normal distribution of active (star-forming) galaxies, all the galaxies would fall linearly in the upper-right, active region. What we wish to determine is which types of environments give rise to a gap.

\subsubsection{Single-Distribution KS Test}

For the single-distribution test, the cumulative distribution function (CDF) of the rotated data was compared with the CDF of a model representing a uniform distribution along the $\mathrm{x}$-axis over the same range as the galaxies. The resulting test yields two values: $D$, which is the maximum deviation of the data from the model, and $\alpha$, which gives the significance level with which it is possible to reject the null hypothesis that the model matches the data. 


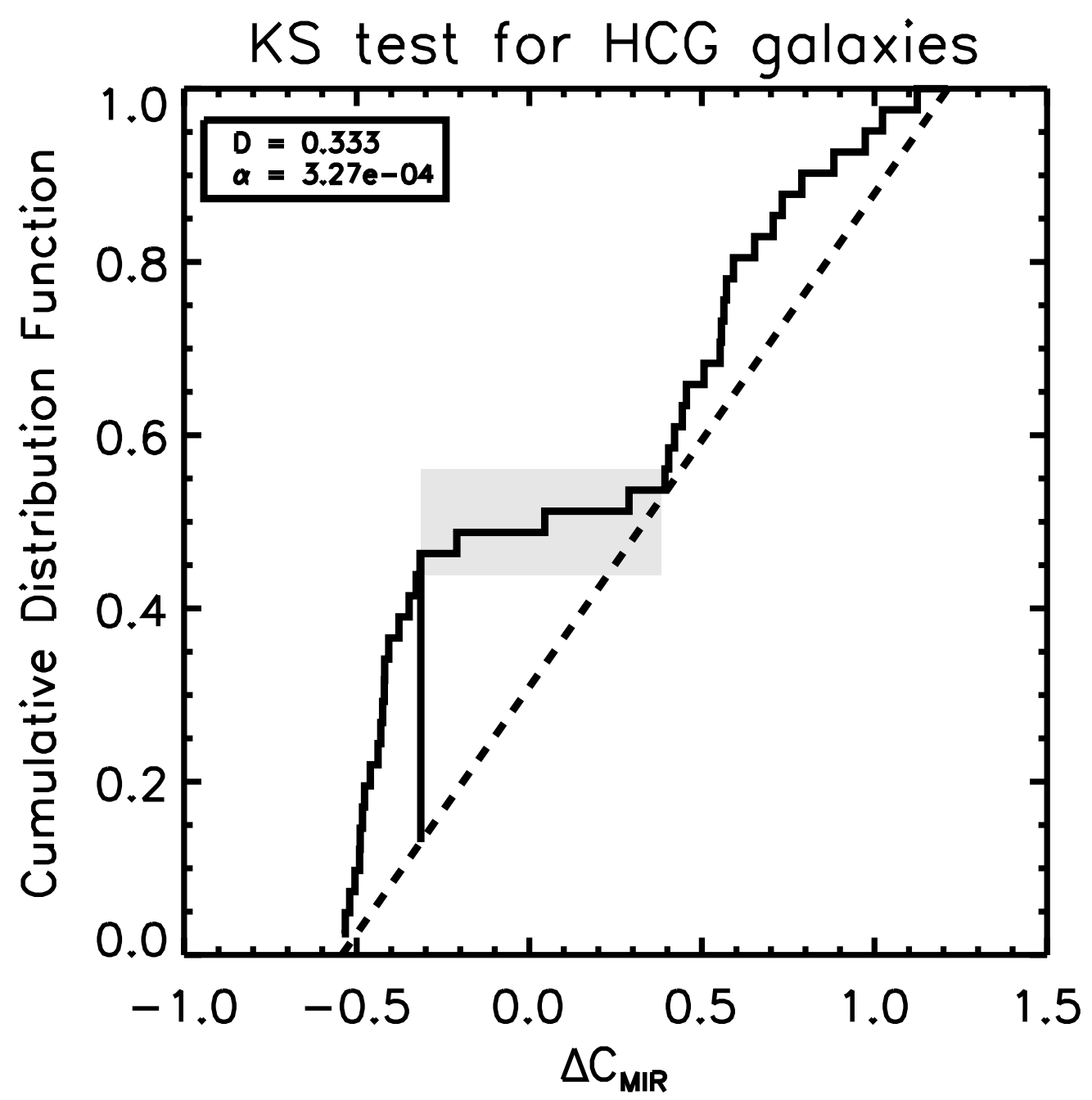

Fig. 2.5. - KS test for the rotated HCG distribution against a model of uniform distribution. The maximum vertical distance between the CDF of the HCG galaxies (solid line) and that expected for a uniform distribution (dashed line) is $D$, indicated by the vertical line. The large value of $D$ indicates that the probability that the HCGs are drawn from a uniform distribution is very low, $0.03 \%$. The nearly flat portion of the CDF highlighted in grey qualitatively reveals the gap. 


\section{HCG Galaxies}

Figure 2.5 shows the single-distribution KS test for the HCG galaxies. It clearly illustrates the gap apparent in Figure 2.3, manifested as the nearly horizontal portion of the CDF over $-0.31<\Delta C_{M I R}<0.38$ indicated by the grey rectangle. The maximum deviation of the sample from the model occurs at the beginning of the gap and is due to the pile-up of galaxies dominated by stellar light. The value of $\alpha$ returned by the test means that it is significant to reject the hypothesis that the HCG galaxies come from a uniform distribution at the $99.97 \%$ confidence level.

\section{Comparison Samples}

As Table 2.2 and Figure 2.6 show, the LVL+SINGS sample is mildly inconsistent with being drawn from a uniform distribution over the color range it covers. The CDF of the interacting sample is concave, indicating that the galaxies are dust-rich and likely gas-rich, forming stars. The CDF of center Coma galaxies, and to a lesser extent the galaxies in the infall region of Coma, is very convex. This is due to the relative overabundance of galaxies with stellar colors, caused by the lack of cold gas and therefore star formation present in these galaxies. Thus the fact that the infall Coma region $\mathrm{CDF}$ is less convex than the center Coma region may indicate that galaxies in this region have not undergone as much processing. From the single-distribution test, it is clear that sample CDFs are affected by environment, as the HCG and Coma samples (i.e. the dense systems) have different CDFs from LVL+SINGS (i.e. the "field" sample). 
Table 2.2. $\quad$ Single-Distribution KS Test

\begin{tabular}{|c|c|c|c|c|c|c|c|}
\hline \multirow[b]{2}{*}{ Sample } & \multicolumn{3}{|c|}{ Cropped } & \multicolumn{3}{|c|}{ Uncropped } & \multirow[b]{2}{*}{ Consistent w/Uniform } \\
\hline & $\mathrm{N}$ & $\mathrm{D}$ & $\alpha$ & $\mathrm{N}$ & $\mathrm{D}$ & $\alpha$ & \\
\hline $\mathrm{HCG}$ & $\ldots$ & $\ldots$ & $\cdots$ & 41 & 0.333 & $3.27 \times 10^{-4}$ & reject \\
\hline LVL+SINGS & 88 & 0.199 & $3.00 \times 10^{-3}$ & 90 & 0.179 & $9.35 \times 10^{-3}$ & do not reject \\
\hline LVL+SINGS (culled) & 67 & 0.236 & $1.72 \times 10^{-3}$ & 68 & 0.238 & $1.37 \times 10^{-3}$ & do not reject \\
\hline Interacting & 57 & 0.376 & $3.12 \times 10^{-7}$ & 58 & 0.375 & $2.79 \times 10^{-7}$ & reject \\
\hline Coma (center) & 128 & 0.622 & $1.60 \times 10^{-43}$ & 155 & 0.378 & $1.96 \times 10^{-19}$ & reject \\
\hline Coma (infall) & 48 & 0.482 & $5.60 \times 10^{-10}$ & 51 & 0.479 & $1.91 \times 10^{-10}$ & reject \\
\hline
\end{tabular}

Note. - The model uniform distribution covers only the range where galaxies are located, and is different for each sample.

Table 2.3. Two-Distribution KS Test

\begin{tabular}{|c|c|c|c|c|c|c|c|c|}
\hline \multirow[b]{2}{*}{ Sample } & \multicolumn{3}{|c|}{ Cropped } & \multicolumn{3}{|c|}{ Uncropped } & \multirow[b]{2}{*}{ Consistent w/HCGs } & \multirow[b]{2}{*}{ Reason } \\
\hline & $\mathrm{N}_{\mathrm{HCGs}}$ & $\mathrm{D}$ & $\alpha$ & $\mathrm{N}_{\mathrm{HCGs}}$ & $\mathrm{D}$ & $\alpha$ & & \\
\hline LVL+SINGS & 38 & 0.406 & $4.24 \times 10^{-4}$ & 41 & 0.399 & $3.42 \times 10^{-4}$ & likely reject & $\mathrm{a}, \mathrm{b}$ \\
\hline LVL+SINGS (culled) & 36 & 0.413 & $8.74 \times 10^{-4}$ & 41 & 0.429 & $2.11 \times 10^{-4}$ & likely reject & $a, b$ \\
\hline Interacting & 21 & 0.459 & $3.52 \times 10^{-3}$ & 41 & 0.574 & $3.11 \times 10^{-7}$ & reject & $\mathrm{b}, \mathrm{c}$ \\
\hline Coma (center) & 40 & 0.414 & $7.87 \times 10^{-5}$ & 41 & 0.441 & $9.01 \times 10^{-6}$ & reject & $\mathrm{a}, \mathrm{c}$ \\
\hline
\end{tabular}

Note. - Cropped covers only the region of colorspace that galaxies from both samples occupy. Uncropped covers the entire region of colorspace that galaxies from either sample occupy. Qualitative reasons for inconsistency: (a) no gap; (b) fundamentally different; (c) covers different range in colorspace, if uncropped. 


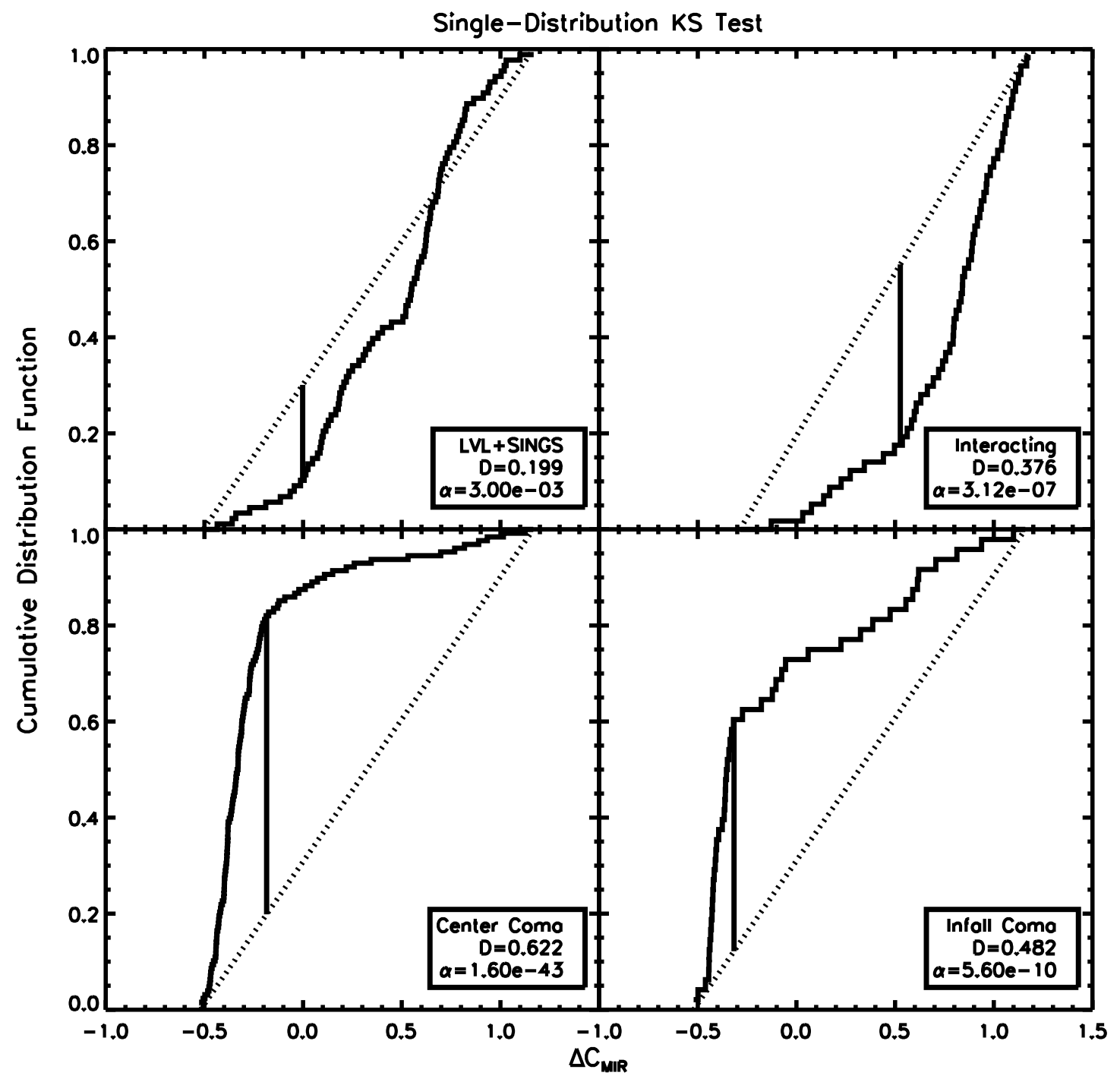

Fig. 2.6. - KS test for the rotated comparison samples against a model of uniform distribution for the cropped samples, with $\mathrm{D}$ and $\alpha$ as defined in Figure 2.5. The LVL+SINGS sample is mildly inconsistent. The interacting sample is concave because the galaxies tend to be gas-rich and mid-IR-bright, and therefore the distribution is weighted towards higher values of $\Delta C_{M I R}$, while the two Coma samples are very convex due to the large fraction of galaxies with stellar colors in the samples. 


\subsubsection{Two-Distribution KS Test}

The two-distribution KS test compared the CDF of each sample against the CDF of the HCG sample. This also calculated $D$ and $\alpha$, with the same meaning as the single-distribution test except that $D$ is now the maximum deviation between the two samples.

The results of the two-distribution KS test are given in Table 2.3 and plotted in Figure 2.7. We deemed a sample inconsistent with the HCG sample if its probability of being consistent with the HCGs was less than 1\%. It is apparent that the distribution of galaxies in the center of Coma is drastically different from the distribution of HCGs, and the distributions for the LVL+SINGS galaxies and interacting galaxies are mildly inconsistent with being drawn from the same distribution as the HCGs. The $\alpha$ value for the infall Coma region sample is high enough that we cannot reject the hypothesis that it is drawn from the same parent distribution. Thus the infall Coma region is most like the HCGs, as it has the smallest $D$ and largest $\alpha$.

\subsubsection{Gap Region}

In order to discover the depth of the gap seen in HCG colorspace, we devised a test to quantitatively discern whether any of the comparison samples exhibit a similar gap. We show the gap region by the grey box in Figure 2.5. This region was defined by the two HCG galaxies bounding the gap, at $\Delta C_{M I R}=-0.31$ and 0.38 . Since the gap represents a deficit of galaxies over this color range, its signature is a flattening in the CDFs. Therefore as another quantitative measure of the gap we performed a linear fit to the CDF between the color boundaries to obtain the slope; shallower slopes indicate samples with more pronounced gaps. The results are given in Table 2.4, which shows that the HCG sample has the most pronounced gap of any of the 


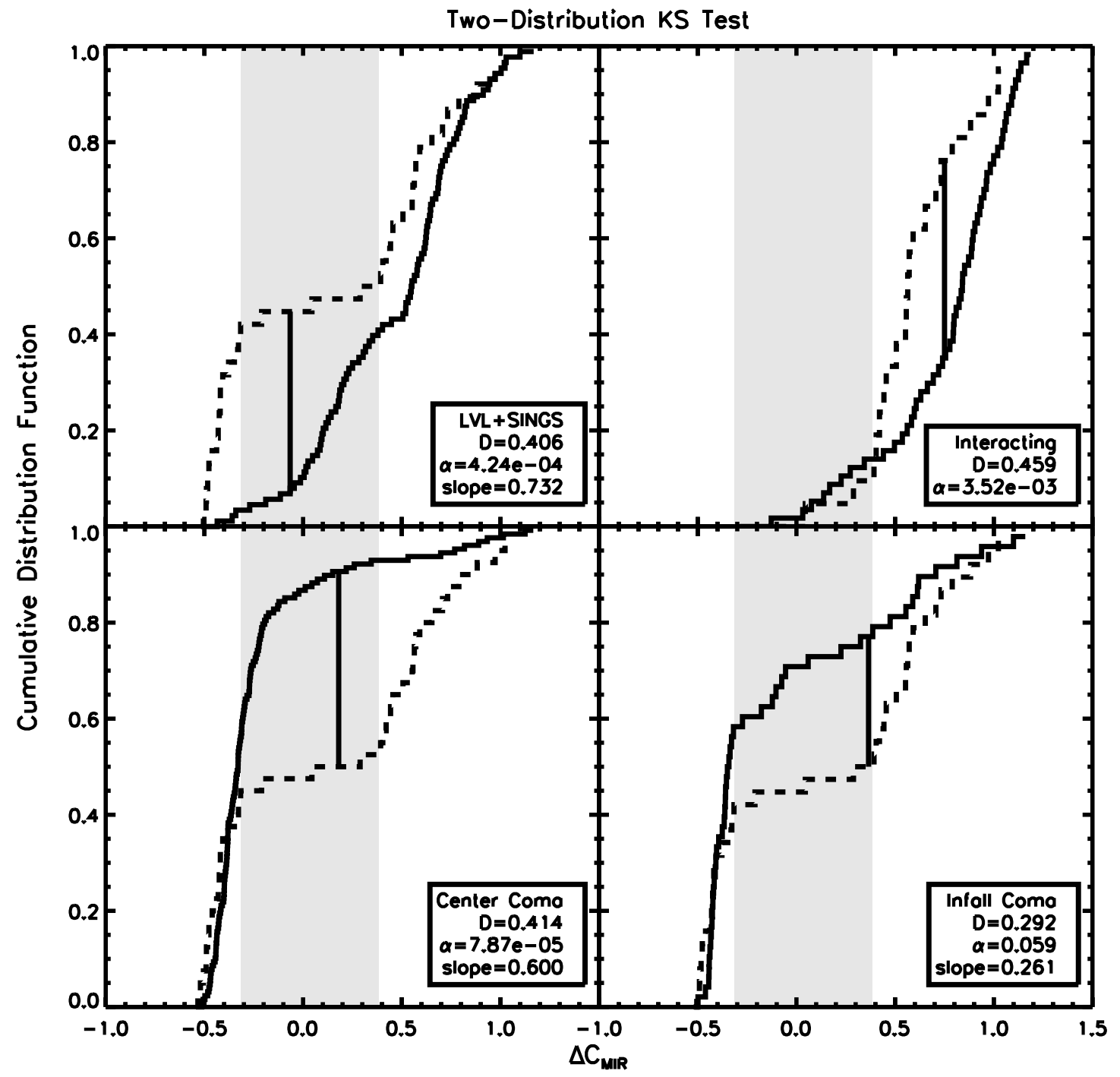

Fig. 2.7. - KS test for the rotated comparison samples against the HCG galaxies for the cropped samples. From the large values of $\mathrm{D}$ and small values of $\alpha$, as defined in Figure 2.5, the LVL+SINGS, interacting, and Coma center samples are clearly inconsistent with the HCG sample. The Coma infall sample has a high enough $\alpha$ that we cannot reject the hypothesis that it is consistent with the HCGs. The slope is a linear fit to the cumulative distribution function over the gap region. 
samples. We have excluded the interacting sample as it begins mid-gap (see Figure 2.4), so a slope would be undefined. The remaining three samples all contain galaxies in the region of stellar colors and thus cover the entire gap region. The slope of the CDF of the infall Coma region over the gap is fairly shallow, possibly indicating the existence of a less-defined gap than the HCG galaxies.

\subsection{Color-Magnitude Diagrams}

Optical color-magnitude diagrams have proven useful for understanding how galaxy luminosity (as a proxy for stellar mass) and color (as an indicator of the level of current star formation) are related. Galaxies are not evenly distributed in this parameter space, but are found in distinct regions: the so-called red sequence, blue cloud, and green valley (Hogg et al. 2004), discussed in §2.7.3. We are interested in investigating whether a color-magnitude diagram using only mid-IR information is similarly helpful in elucidating galaxy properties.

We created a plot analogous to a color-magnitude diagram using the data discussed in $\S 2.4$. A comparison to the optical red sequence will be discussed in $\S 2.7$. Figure 2.8 shows the rotated color-luminosity diagram (hereafter referred to as CMD) for the HCGs, with the gap region again highlighted in grey. The color distribution and gap seen in Figure 2.3 are clearly apparent in Figure 2.8. Within the HCG sample, a galaxy's luminosity does not appear to depend on its color. Note that even if we relax the luminosity cut $\left(\log \left(L_{4.5}[\mathrm{erg} / \mathrm{s}]\right) \geq 40.9\right)$, the gap is still quite obvious. Galaxies to the left of the gap have blue mid-IR colors, indicating that there is no PAH or dust emission and that the mid-IR SED is consistent with stellar photospheric colors. On the other side of the gap are galaxies with red mid-IR colors, which means that the SED is dominated by PAHs and warm/hot dust. Galaxies in the gap region would 


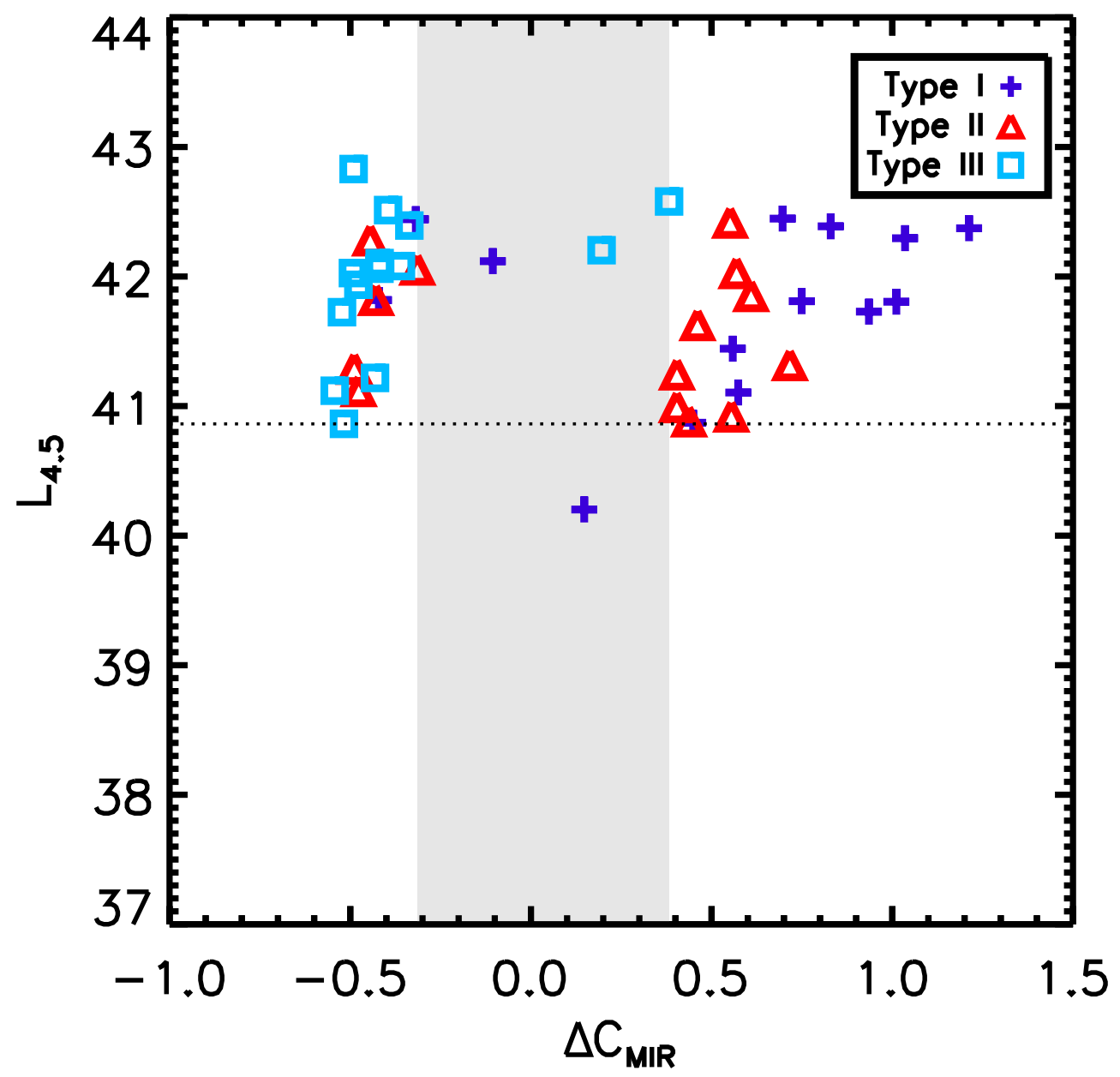

Fig. 2.8. - CMD for the HCG galaxies. The dotted line indicates the minimum luminosity required for inclusion. The gap is still apparent, indicated by the grey stripe. Notably, there is no evidence for a color-luminosity correlation. 
have weak PAH/dust emission compared to starlight. The trend with H I type is the same as seen in Figure 2.3: galaxies from H I-rich groups (Type I) generally have "active" (red) mid-IR colors, while galaxies from H I-poor groups (Type III) generally have "passive" (blue) mid-IR colors. Galaxies from Type II groups fall on both sides of the gap. Interestingly, galaxies within the gap are from either Type I or Type III groups, but not Type II, but given the small number statistics, this may not be significant.

The CMDs for the comparison samples are shown in Figure 2.9. Like the HCGs, the luminosities of galaxies in the interacting and both Coma samples also do not appear to be related to color over the range of the sample. However, the LVL+SINGS sample does show a correlation between luminosity and color - the brighter galaxies tend to have mid-IR-redder colors (with the exception of the 'tail'). Thus LVL+SINGS has primarily faint, mid-IR-blue (inactive) galaxies and bright, mid-IR-red (active) galaxies.

As with the color-color plots, the shape of the CMDs reflects the criteria used to define each sample. For example, the center Coma and infall Coma samples have a significant concentration of galaxies with blue mid-IR colors. These colors indicate SEDs that fall from 3 to $8 \mu \mathrm{m}$, consistent with stellar light arising from the tail of the Raleigh-Jeans distribution, and a general lack of emission from the interstellar medium. The interacting sample, on the other hand, is strongly biased towards actively star forming systems. Therefore its CMD lacks a concentration of massive galaxies with blue mid-IR colors. Instead, it shows a scattering of galaxies with red mid-IR colors, indicative of PAH features associated with active star formation or SEDs that rise to longer wavelengths due to the presence of warm dust.

The CMD of the HCGs is a composite - it contains both a concentration at blue 


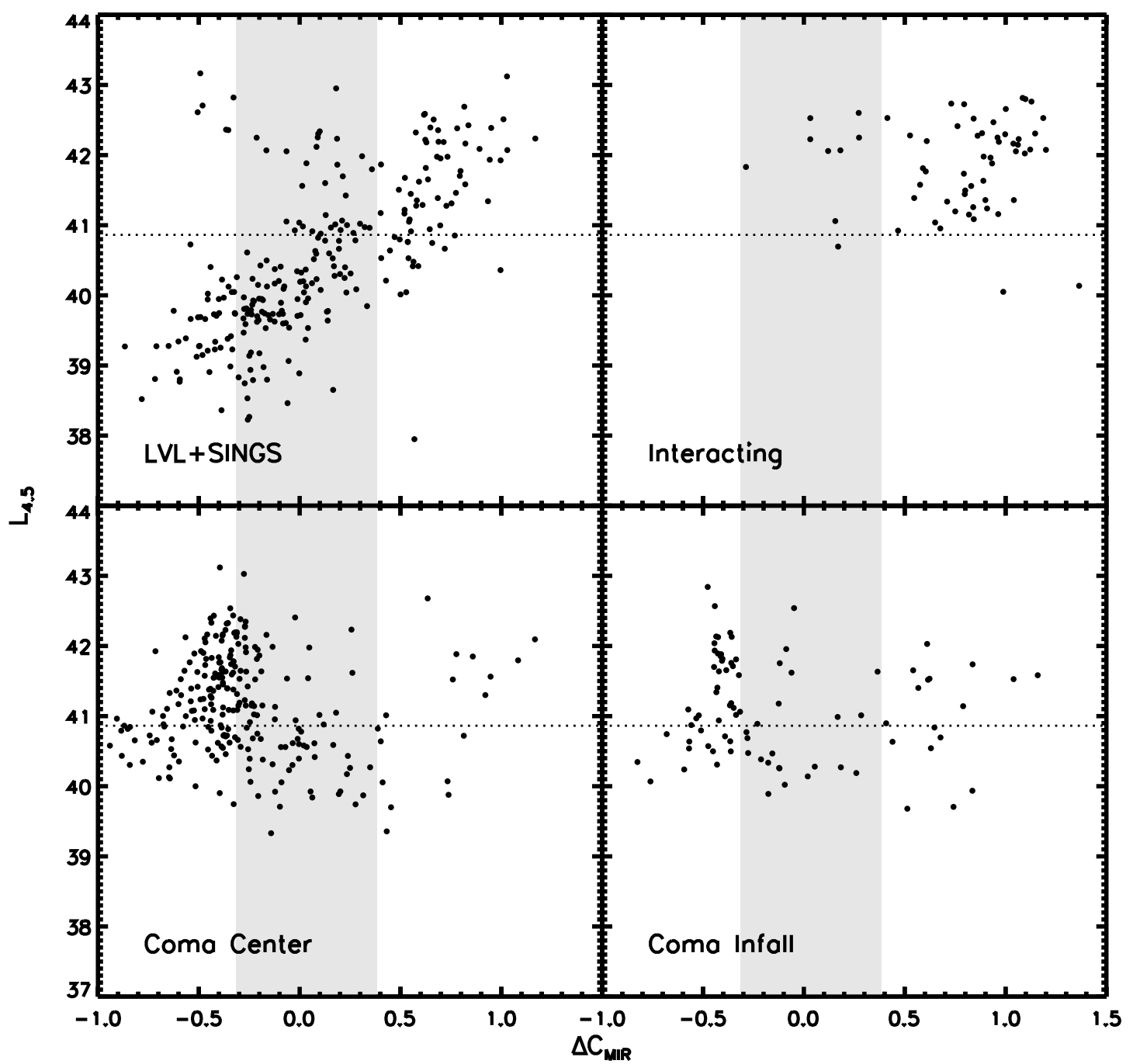

Fig. 2.9.- CMD for the comparison samples. As in Figure 2.8, the dotted line indicates the luminosity cut imposed on the samples, and the HCG gap is highlighted by the grey stripe. The criteria used to define each sample are apparent in the shape of the CMDs. LVL+SINGS is the only sample which shows color dependence on luminosity, discussed further in the text. 
mid-IR colors, as well as a scattering of systems with red mid-IR colors, but very few galaxies with intermediate mid-IR colors. As suggested by the KS tests, the HCG CMD distribution is most similar to that of the sample from the Coma Infall region, and quite unlike that of the other samples.

\subsection{Properties of Galaxies along Mid-IR Colorspace}

\subsubsection{Morphology}

In an attempt to determine the properties of galaxies as a function of mid-IR colorspace, as well as the physical origin of the gap, we plotted the distribution of morphologies (obtained from Johnson et al. (2007); Dale et al. (2007, 2009)) for the HCG and LVL+SINGS galaxies in colorspace, as shown in Figure 2.10. Interestingly, the LVL+SINGS colors appear to be independent of morphology, while the colors of the HCG sample show a morphological segregation. For the HCG sample, the two galaxies that fall in the gap are Sab and Im. The LVL+SINGS sample shows a variety of morphological types in the gap. We considered the possibility that the lack of correlation was due to the diversity of SINGS galaxies, but when we remove the SINGS sample, the same lack of correspondence is seen in the LVL sample. For the culled SINGS sample of Smith et al. (2007), we also do not see a strong correlation between Hubble type and Spitzer IRAC colors.

We find this result both surprising and puzzling, especially given previous studies which have found a trend in mid-IR color with Hubble type (Pahre et al. 2004) and classical work by Kennicutt \& Kent (1983) that shows a correlation between $\mathrm{H} \alpha$ equivalent width and Hubble type. We have investigated the most extreme exam-

ples of this lack of morphological segregation - the mid-IR red E/S0 galaxies and the 
Table 2.4. CDF Slopes in Gap Region

\begin{tabular}{ccc}
\hline \hline & \multicolumn{2}{c}{ Slope } \\
Sample & Cropped & Uncropped \\
\hline HCG & 0.104 & $\ldots$ \\
LVL+SINGS & 0.732 & 0.743 \\
LVL+SINGS (culled) & 0.896 & 0.883 \\
Interacting & $\ldots$ & $\ldots$ \\
Coma (center) & 0.600 & 0.518 \\
Coma (infall) & 0.261 & 0.246 \\
\hline
\end{tabular}

Note. - Slopes of the CDFs of all the samples over the region of the gap in the HCG sample.
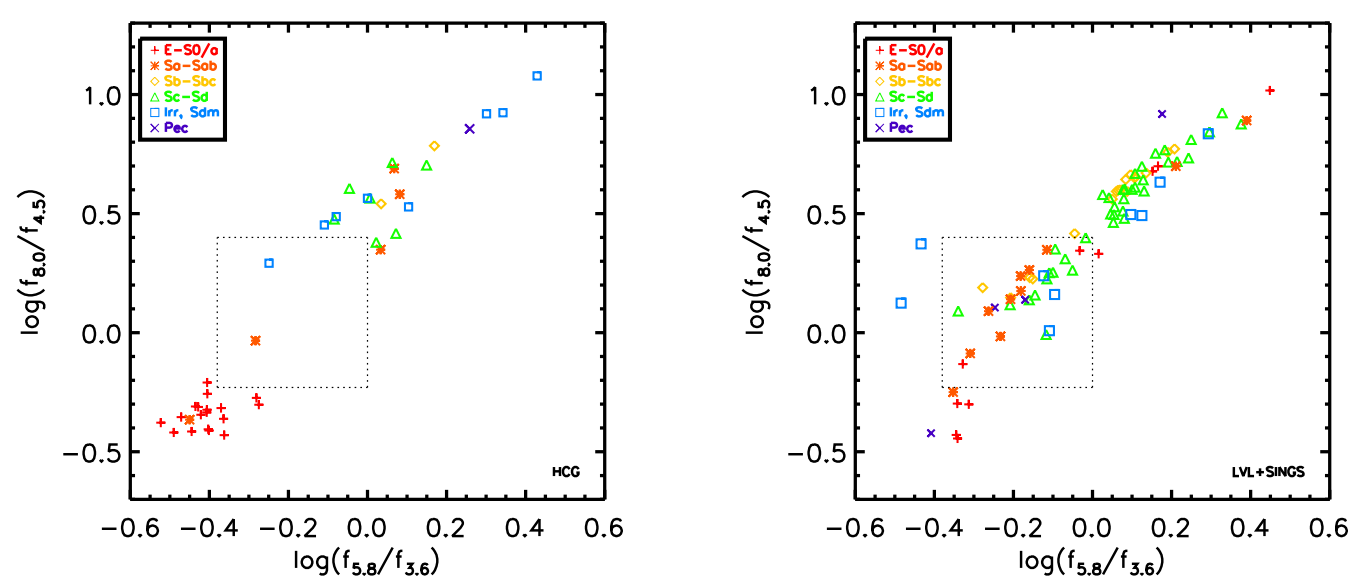

Fig. 2.10.- Distribution of galaxy morphology in mid-IR colorspace for left: HCGs and right: LVL+SINGS. Note that the distribution of morphologies for the HCGs is as expected, with E/S0 galaxies occupying the lower left and spiral galaxies occupying the upper right. This is not strictly the case for LVL+SINGS. 
$\mathrm{Sm} / \mathrm{Sdm}$ galaxies which fall in the gap. We found that the blue early type galaxies were all starbursts (see §2.6.2), while the red late type galaxies are low surface brightness galaxies (i.e. NGC 45, 4656, and 5398; Monnier Ragaigne et al. 2003), have low metallicities (i.e. NGC 55; Jackson et al. 2006), or are Seyferts which exhibit large flux variations (i.e. NGC 4395; Minezaki et al. 2006). One possible explanation is that the environment present in compact groups causes morphology to more closely track the activity level of a galaxy than in the field. We hypothesize that this could be due to the presence or absence of neutral gas - if there is gas present, the galaxies will be actively forming stars, which will typically be visible as a disk or irregular galaxy. If most of the gas has been used up, there will be no star formation occurring, and the galaxy will typically be an elliptical or lenticular. In the field, the amount of gas and level of star formation do not appear to be as closely connected, so the galaxy types do not show the segregation seen in the HCG galaxies.

Figure 2.11 shows Hubble type as a function of rotated mid-IR color for the HCG galaxies and LVL+SINGS sample. This plot clearly reveals a dearth of galaxies over $2<T<6$ (S0/a-Sbc) for the HCGs, seen previously in Figure 2.2. Of the few galaxies with these Hubble types, they do not preferentially fall in the gap. The LVL+SINGS galaxies do not show a dearth of galaxies between these Hubble types. In addition, almost every Hubble type appears in the gap. This begs the question of whether the existence of the gap is nature or nurture. The selection criteria for the HCGs may have for some reason selected against these morphological types. Alternatively, there might something about the HCG environment that suppresses these Hubble types or alters their SFR so that they do not fall in the gap. 

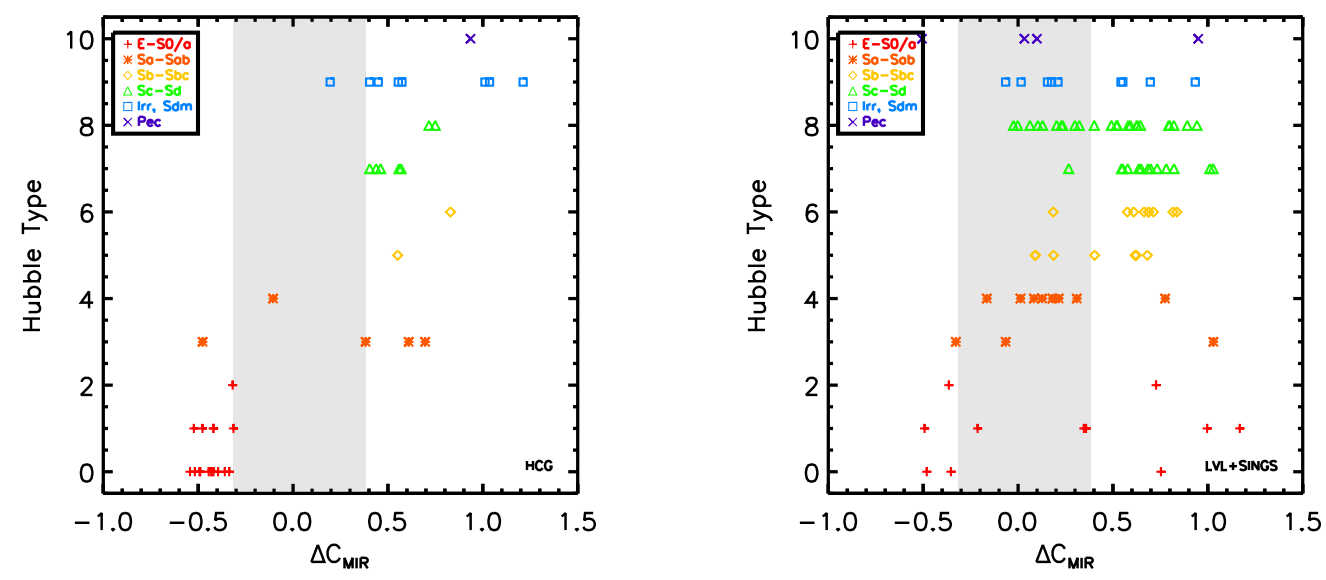

Fig. 2.11. - Distribution of galaxy morphology in rotated mid-IR colorspace for left: HCGs and right: LVL+SINGS. Note the dearth of morphologies over $2<T<6$ in the HCG sample.
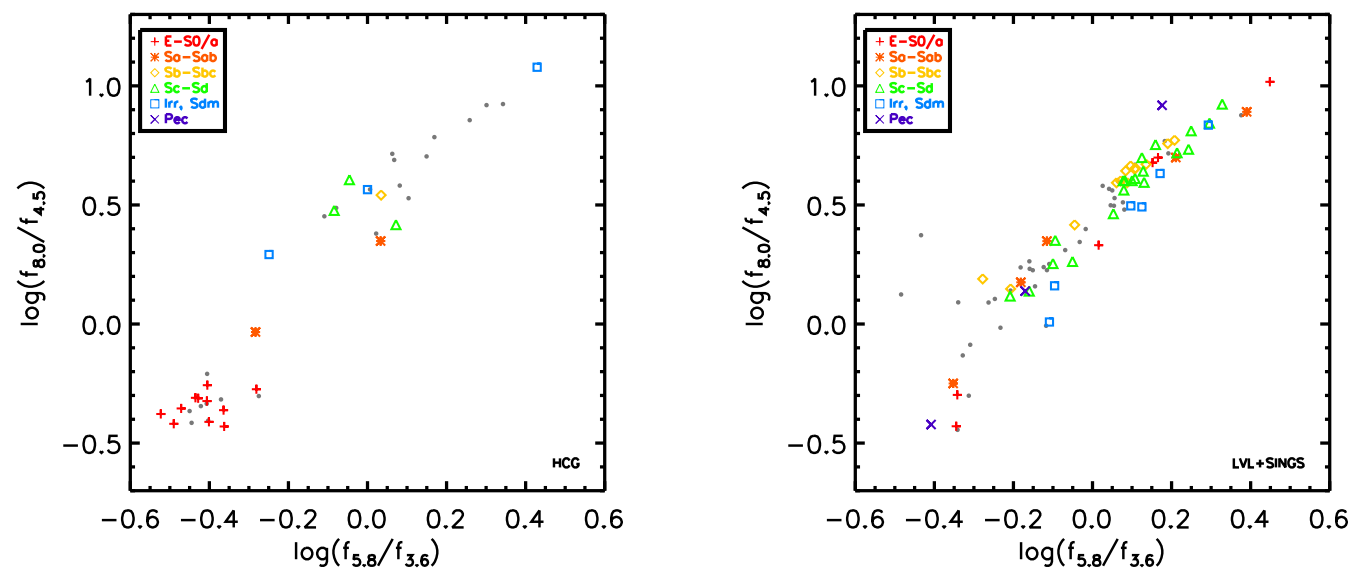

Fig. 2.12.- IRAC colorspace distribution for left: HCGs and right: LVL+SINGS. Galaxies for which spectra were obtained are identified by their morphological type. Galaxies for which spectra were unavailable are shown as grey dots. These plots show that spectra sample the full range of colorspace. 


\subsection{2 $\mathrm{EW}(\mathrm{H} \alpha)$ and Specific Star Formation Rates}

The emission (or absorption) of Balmer lines tracks the recent star formation history of a galaxy through ionized gas, and is complimentary to the mid-IR data which trace warm dust emission. In order to gain more insight into the properties of star formation in the gap region, here we looked at the $E W(H \alpha)$ as a function of $\Delta C_{M I R}$. For four groups in our HCG sample (HCGs 7, 42, 62, 90), we have obtained Hydra CTIO spectra with $2^{\prime \prime}$ fibers located on the nuclei. For many of the galaxies in the SINGS sample, optical spectra were obtained with $2.5 \times 2.5^{\prime \prime}$ slits located on the nuclei (Moustakas \& Kennicutt 2006). These data are publicly available at http://irsa.ipac.caltech.edu/data/SPITZER/SINGS/. In addition to these two datasets, many of the galaxies in both the HCG galaxies and in the LVL+SINGS sample have spectra available through SDSS.

For all of these galaxies, we determined the equivalent width of the $H \alpha$ line using Gaussians in the SPLOT task in IRAF. Figure 2.12 shows the morphologies and locations in colorspace of the galaxies for which we were able to obtain spectra. A plot of $E W(H \alpha)$ vs $\Delta C_{M I R}$ is shown in Figure 2.13. In both the HCG sample and the LVL+SINGS sample, all galaxies bluewards of the gap have $E W(H \alpha) \lesssim 0$, which is fully consistent with mid-IR colors indicating predominantly stellar emission. Galaxies redward of the gap exhibit a large range of $E W(H \alpha)$, but there is not necessarily a one-to-one correspondence with mid-IR emission. This could be explained by several scenarios: 1) dust absorption of $H \alpha$ and general effects of relative geometry; 2) variable amounts of PAH emission; 3) different timescales traced by $H \alpha$ and midIR colors; 4) a coverage difference - the mid-IR colors are integrated over the entire galaxy, while the $H \alpha$ was measured only in the nuclear region. For a given $\Delta C_{M I R}$, the galaxies in the LVL+SINGS sample cover a larger range in $E W(H \alpha)$ than the 
HCG galaxies. This is likely because the galaxies in the LVL+SINGS sample have varying amounts of dust - in fact, the SINGS sample was chosen to be as diverse as possible (Kennicutt et al. 2003). All galaxies in the HCG sample have $E W(H \alpha)$ consistent with their Hubble type (Nakamura et al. 2004). For LVL+SINGS, there are several galaxies which have $E W(H \alpha)$ inconsistent with their Hubble type - there are peculiar galaxies with $E W(H \alpha) \sim 0$ and E/S0 galaxies with large $E W(H \alpha)$. In both samples however, the $E W(H \alpha)$ seems to exhibit an upper envelope, that changes as a function of $\Delta C_{M I R}$.

As galaxies in the gap have similar $E W(H \alpha)$ in both the HCG and LVL+SINGS sample, the properties of the gap galaxies are likely similar. Hence, the HCG sample must be deficient in a certain type of galaxy, rather than having galaxies with fundamentally different properties. Since the gap is between galaxies with colors consistent with stellar photospheric emission and galaxies with relatively high amounts of star formation, we suggest that the gap is likely caused by a deficit of galaxies with moderate specific star formation rates (SSFR). In order to investigate this, we took the SSFRs from Tzanavaris et al. (2010), computed as

$$
S S F R=\frac{9.5 \times 10^{-44} \nu L_{\nu, u v w 2}[\mathrm{erg} / \mathrm{s}]+2.14 \times 10^{-42} \nu L_{\nu, 24 \mu \mathrm{m}}[\mathrm{erg} / \mathrm{s}]}{M_{*}}
$$

and plotted them against mid-IR color, as shown in Figure 2.14. As is clearly visible from the figure, the mid-IR gap corresponds perfectly with the gap in SSFR. Thus the HCG environment appears to be inhospitable to galaxies with moderate SSFRs $\left(3.2 \times 10^{-11}<S S F R<1.2 \times 10^{-10} \mathrm{yr}^{-1}\right)$. 


\subsection{Discussion}

\subsubsection{The relationship between environment and presence of gap}

Our main result is that the HCG sample is statistically different from the comparison samples in mid-infrared (mid-IR) colorspace, cumulative distribution functions (CDFs), and rotated color-luminosity diagrams (CMDs), due to the presence of a gap in mid-IR colorspace. Of the other samples considered here, the HCG sample is most like the infall Coma sample, and unlike the interacting sample, the center Coma sample, or the LVL+SINGS sample. While the HCG sample is most like the infall Coma sample, the lack of galaxies with transitional mid-IR colors is more pronounced in the HCG sample.

The fact that the HCG sample is most like the infall Coma sample is unsurprising,

as other similarities between the compact group environment and the outskirts of clusters have been seen. Lewis et al. (2002) found suppression of SFRs in clusters out to three virial radii, and determined that star formation suppression depends more on local galaxy density than on overall cluster properties. In addition, studies by Cortese et al. (2006) of a compact group falling into a cluster suggest that the galaxies in the group have undergone pre-processing due to the local compact group environment.

\subsubsection{Possible mechanisms for Mid-IR gap}

We have established the statistical significance of the gap in $\S 2.4$, however the origin and nature of the gap remains an important issue; here we consider three possibilities. The first is that it arises from a fluctuation due to small number statistics; however, the $\mathrm{KS}$ tests presented in $\S 2.4$ conclusively demonstrate that the $\mathrm{HCG}$ sample is 
inconsistent with being drawn from a uniform distribution, even taking into account sample size. The second possibility is a subtle selection effect by either us or Hickson, a possibility that can be investigated by including more HCGs in the analysis as well as expanding the sample to include Redshift Survey Compact Groups (RSCGs; Barton et al. 1996), though it seems unlikely that a selection effect could cause the observed gap. In other words, this would require a bias against galaxies with moderate SSFR, but not low or high SSFR. The third possibility is that the paucity of morphological types could correspond to the mid-IR gap. The morphology histogram (Figure 2.2) shows a dearth of galaxies between $2 \geq T \geq 6$. For the HCG sample it is impossible to determine what is not in the gap, but we can look at gap galaxies in LVL+SINGS for insight. Since LVL+SINGS shows galaxies of all types in the gap, it is not consistent with morphological type being a predominant factor in creating the gap. However, it is possible that HCGs select against galaxies that would fall in the gap. This leads us to the possiblity that the gap is due to a deficit of galaxies exhibiting moderate specific star formation rates due to environmental effects of the CG environment.

We conclude that the distribution in colorspace, and in particular the occurence of a gap in the mid-IR color distribution most likely reflects different levels of specific star formation, with galaxies ranging from active (red mid-IR colors) to passive (blue midIR colors). This is supported by the gap seen in the SSFRs for these HCG galaxies, independently determined using UV, $24 \mu \mathrm{m}$, and K-band data (Tzanavaris et al. 2010). The distribution in colorspace suggests that galaxies in the HCG environment spend little time in the evolutionary state that yields mid-IR colors in the gap region. The fact that the gap is seen in both the HCG and Coma infall samples, but is more pronounced in the HCG sample indicates that this effect is similar to, but more dramatic than, the environment in the outskirts of clusters. The tidal fields present 
in HCGs can easily funnel gas to the inner parts of galaxies, inducing high levels of star formation. Once this gas is used up or heated to high temperatures, the galaxies can no longer form stars, and quickly become quiescent. Thus galaxies in HCGs either experience a profusion of star formation or do not have the gas necessary to form stars, and do not exhibit moderate levels of star formation.

\subsubsection{Relationship to optical CMDs}

Insight into the shape of mid-IR CMDs may be gained by analogy to optical CMDs. In the latter, several prominent features have been identified - an optical "red sequence" of galaxies with a relatively narrow distribution of red optical colors, extending to the brightest magnitudes; an optical "blue cloud" of generally fainter galaxies with bluer optical colors (e.g. Strateva et al. 2001; Hogg et al. 2004); and a deficit of galaxies with intermediate optical colors (the so-called "green valley"; Hogg et al. (2004)). Optical red sequence galaxies are dominated by E/S0 galaxies, while optical blue cloud galaxies are dominated by disk and irregular systems. This optical CMD shape is apparent in both dense and sparse environments, although dense environments have a more prominent red sequence, while less dense environments have a more pronounced blue cloud.

The optical CMD shape is often interpreted in terms of evolutionary processes, in which optical blue cloud galaxies might evolve onto the red sequence through some combination of wet and dry merging, star formation, star formation "quenching", and aging (e.g. Faber et al. 2007, and references therein). A key attribute of such models is that galaxies must move rapidly between the optical blue and red phases in order to reproduce the red/blue dichotomy (e.g. Dekel \& Birnboim 2006). The optical "green valley" region therefore includes these evolutionary transitional systems. However, 
the optical red sequence is contaminated by dusty star-forming galaxies. This is a significant advantage of the mid-IR over optical.

The interpretation of the mid-IR CMDs is expected to be similar to that of the optical CMDs, however discussing mid-IR and optical CMDs simultaneously can be confusing because of the different connotations of "blue" and "red". In optical colors blue light typically reflects a young population and the optical red sequence picks out both "red and dead" galaxies (i.e. galaxies whose colors are dominated by evolved stars and have little or no active star formation (e.g. van Dokkum 2005; Bell et al. 2004)) and galaxies obscured by dust, which may be active. On the other hand, midIR colors reflect the properties of the dust, so blue mid-IR galaxies select for systems with little dust contribution, which are dominated by stellar photospheric emission (whether from young or old stars), with little or no active star formation. Blue mid-IR colors therefore select both "optically red and dead" and "optically blue and dying" galaxies. Red mid-IR galaxies are active, with current star formation heating the dust and exciting the PAHs, so they are most closely related to the optical blue cloud. Additionally, the optical green valley is present in all environments (Hogg et al. 2004), while we have only found evidence for the mid-IR gap in dense environments that still contain neutral gas.

\subsubsection{The role of gas in CG evolution}

The presence of neutral gas may be a key factor contributing to the gap in mid-IR colorspace. Without fuel for star formation, the mid-IR colors would be dominated by stellar emission. There are currently two theories on H I distribution within HCGs: the first is that groups whose H I is contained entirely within the member galaxies are less evolved or younger in stellar population than those groups with 
$\mathrm{H}$ I that is distributed within and between member galaxies (cf. Williams et al. 1990; Verdes-Montenegro et al. 2001); the second is that the distribution of H I determines how HCGs evolve, i.e. HCGs whose H I is contained entirely within the galaxies evolve differently than HCGs whose H I is distributed throughout the group (Konstantopoulos et al. 2010). Thus the H I properties of a group are a crucial component related to a galaxy's mid-IR color. HCG galaxies from H I-rich groups (Type I) primarily lie redward of the gap, while galaxies from H I-poor groups (Type III) primarily lie blueward. Galaxies from Type II groups lie both redward and blueward of the gap, while avoiding it. Curiously, the few galaxies within the gap are from either Type I or Type III groups. Further investigation into this trend will necessitate interferometric H I observations of compact groups, in order to determine the H I deficiency (and thus type) of individual galaxies within compact groups. H I imaging will reveal whether "rogue" galaxies (e.g. mid-IR-red galaxies from Type III groups) have a different individual deficiency from their group as a whole. Interferometric observations will also allow us to determine where the $\mathrm{H}$ I is located whether it is confined to the member galaxies, or distributed throughout the group. H I distribution could be another clue in compact group evolution.

One estimate of the timescale of the proposed rapid evolution in SSFR is the time it takes a galaxy to use up the available gas and go from starburst to poststarburst. Thus this could occur on timescales as short as a million years (Gao \& Solomon 1999), but will clearly be highly dependent on environment. A crude upper limit (assuming $100 \%$ efficiency and a constant SFR), using the H I mass of the group divided by the sum of the SFRs in each member galaxy yields gas depletion timescales ranging from 0.4 Gyr to 4 Gyr.

The presence of the gap in the mid-IR colorspace distribution of the HCGs com- 
bined with the fact that the gap is not present in less dense environments indicates that local environment significantly influences galaxy properties. In order to understand the processes that affect galaxy evolution, we need to understand how gas is processed in the interstellar medium and intragroup medium distinct from the field and cluster environments. Compact groups are clearly an important part of understanding galaxy evolution and cluster assembly, especially considering the similarities between the distribution in colorspace of the HCGs and the Coma infall region. 

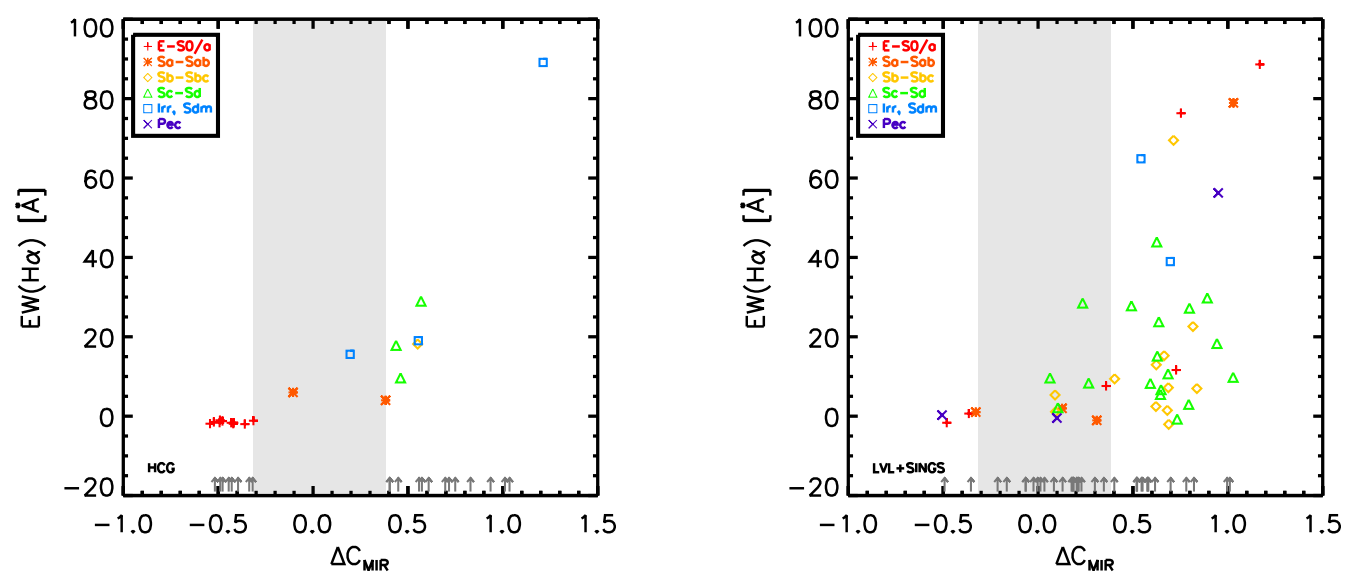

Fig. 2.13.- $E W(H \alpha)$ vs $\Delta C_{M I R}$ for left: HCGs and right: LVL+SINGS. Galaxies for which spectra were unavailable are plotted as dark grey dots at the bottom. The gap is identified by the grey stripe. In both samples, there is a clear difference between galaxies blueward of the gap and those redward of the gap. 


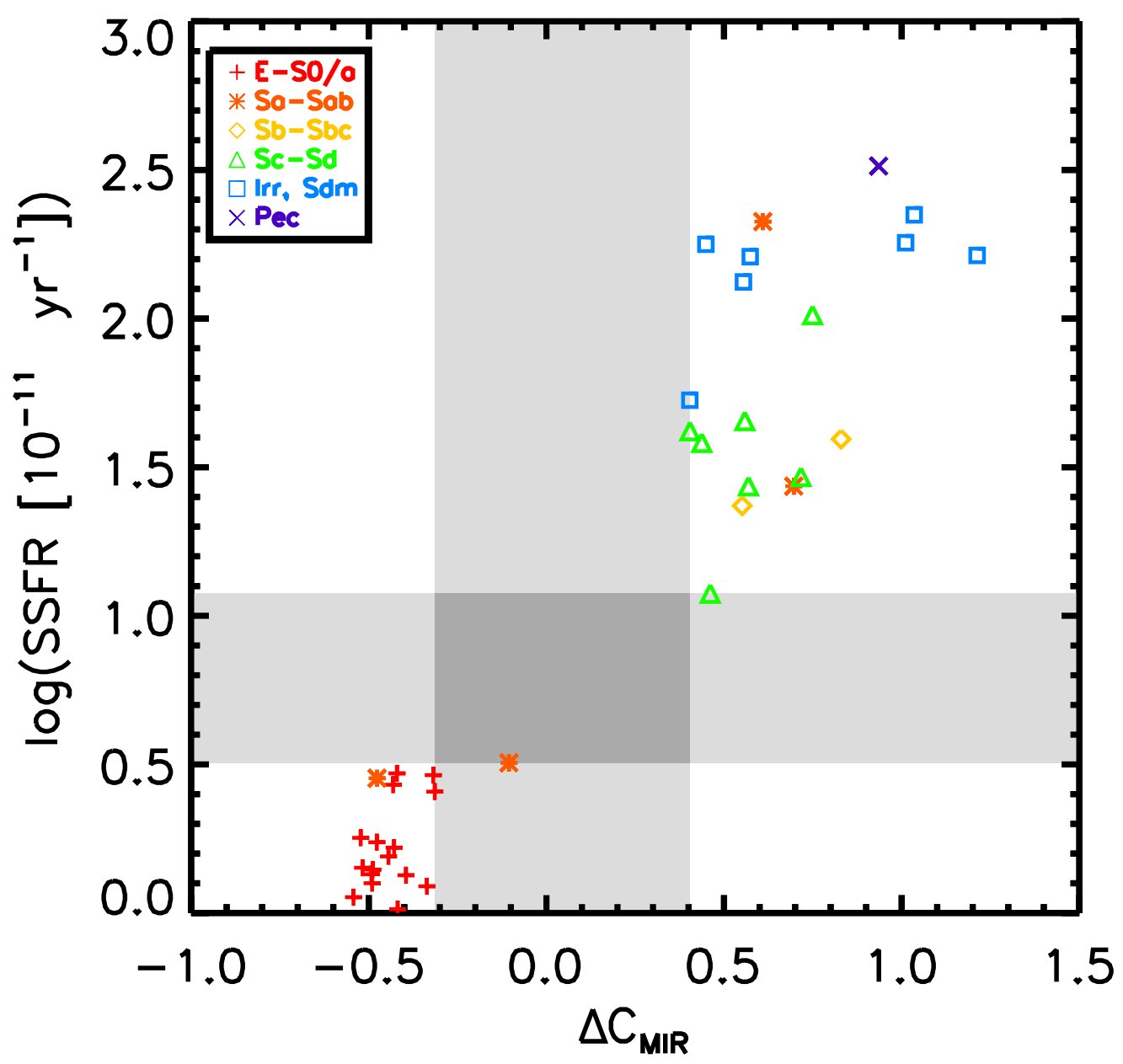

Fig. 2.14. - SSFR (from Tzanavaris et al. 2010) vs $\Delta C_{M I R}$ for 11 of the 12 HCGs in our sample (HCG 90 was excluded due to insufficient data for determining SSFRs). The vertical grey stripe indicates the gap seen in mid-IR colorspace, the horizontal grey stripe indicates the gap in SSFR. 
Chapter 3

Examining the Role of

Environment in a Comprehensive Sample of Compact Groups 


\section{Abstract}

Compact groups, with their high number densities, small velocity dispersions, and an interstellar medium that has not been fully processed, provide a local analog to conditions of galaxy interactions in the earlier universe. The frequent and prolonged gravitational encounters that occur in compact groups affect the evolution of the constituent galaxies in a myriad of ways, for example gas processing and star formation. Recently, a statistically significant "gap" has been discovered mid-infrared (mid-IR: 3.6- $8 \mu \mathrm{m})$ IRAC colorspace of compact group galaxies. This gap is not seen in field samples and is a new example of how the compact group environment may affect the evolution of member galaxies. In order to investigate the origin and nature of this gap, we have compiled a larger sample of 37 compact groups in addition to the original 12 groups studied by Johnson et al. (2007) (yielding 174 individual galaxies with reliable mid-IR photometry). We find that a statistically significant deficit of galaxies in this gap region of IRAC colorspace is persistant in the full sample, lending support to the hypothesis that the compact group environment inhibits moderate SSFRs. Using this expanded sample, we have more fully characterized the distribution of galaxies in this colorspace and quantified the low density region more fully with respect to mid-IR bluer and mid-IR redder colors. We note a curvature in the colorspace distribution, which is fully consistent with increasing dust temperature as the activity in a galaxy increases. This full sample of 49 compact groups allows us to subdivide the data according to physical properties of the groups. An analysis of these subsamples indicates that neither projected physical diameter nor density show a trend in colorspace within the values represented by this sample. We hypothesize that the apparent lack of a trend is due to the relatively small range of properties in this sample, whose groups have already been pre-selected to be compact and dense. 
Thus, the relative influence of stochastic effects (such as the particular distribution and amount of star formation in individual galaxies) becomes dominant. We analyze spectral energy distributions of member galaxies as a function of their location in colorspace and find that galaxies in different regions of mid-IR colorspace contain dust with varying temperatures and/or PAH emission.

This project is an extension of my first project. A significant difference in terms of my contribution is that I performed the photometry and chose the sample, expanding from the HCG catalog to also include groups from the RSCG catalog. I then converted the photometry to physically meaningful fluxes and performed the analysis, which is quite similar to the previous project. However, I did discover the curvature in mid-IR colorspace not present in the previous sample and investigate it through toy models of stars and mid-IR spectra (these spectra were reduced by collaborator David Whelan). I quantitatively identified the canyon region and expanded the statistical tests from my previous work to also include measuring the slope of the cumulative distribution function in the canyon region. I investigated the effect of different group properties on colorspace location of member galaxies. Finally, I wrote the chapter.

\subsection{Introduction}

Recently, Walker et al. (2010) examined the distribution of galaxies from 12 compact groups in IRAC (3.6-8.0 $\mu \mathrm{m})$ colorspace and found evidence for a statistically significant gap between the location of galaxies with colors consistent with normal stellar populations and galaxies with colors indicative of star formation activity (previously identified by Johnson et al. 2007; Gallagher et al. 2008). In contrast, no gap is seen in the colorspace distribution of the core of the Coma cluster, a control sample of field galaxies, or a sample of interacting galaxies. While this is not surprising, the 
varying distributions in IRAC colorspace indicate that these environments have had a significant impact on the evolution of their galaxies. However, a similar (though less pronounced) gap was observed in the outer regions of the Coma cluster. This is evidence that the gap is unique to regions of enhanced galaxy density (relative to the field) in which the neutral gas has not been fully processed.

The discovery of the gap in IRAC colorspace naturally leads to the question of the origin of the gap, which has been intractable due to the small size of the previous sample. Initial results comparing the IRAC gap with specific SFRs (SSFRs; Tzanavaris et al. 2010) of the same sample of groups show that the gap in IRAC colorspace corresponds to a gap in SSFRs (Walker et al. 2010), suggesting a shared underlying cause. In order to better investigate the region of colorspace identified as the gap, we need to increase the number of observed compact group galaxies, so as to understand the characteristics of any systems that may be found in this region as well as characterize any correlation with physical properties along the sequence in colorspace. In order to further populate IRAC colorspace, in this paper we present the colors of galaxies for a large sample of Hickson Compact Groups (HCG; Hickson 1982) and Redshift Survey Compact Groups (RSCG; Barton et al. 1996) at redshifts of $z<0.035$ with a full suite of IRAC data available from the Spitzer Space Telescope archive, yielding 49 compact groups including 179 galaxies.

In this paper, we adopt the following terms: the "original sample" is the 12 HCGs studied in Johnson et al. (2007) and subsequent papers. The "new sample" consists of the 21 HCGs and 16 RSCGs not previously studied by this group. The "full" or "expanded sample" is the combined original and new samples. 


\subsection{Data}

\subsubsection{Samples}

\section{Compact Groups}

Because the goal of this project is to understand galaxy evolution in the dense environment of compact groups, we began with the HCG and RSCG catalogs. There are several groups that appear in both the HCG and RSCG catalog; naturally these groups have only been included once in the full sample. Including the RSCGs in the sample will allow future analysis of the effect of varying group properties and environments on the member galaxies.

For this study, the HCG and RSCG catalogs were sub-selected to only include compact groups at a low enough redshift $(z<0.035)$ such that the polycyclic aromatic hydrocarbon (PAH) features do not shift out of their rest-frame bands, which would make an analysis of their distribution in colorspace infeasible. We searched the Spitzer archive for groups meeting this criterion, and included groups for which all four channels of IRAC data are available. This yielded 21 HCGs (in addition to the original sample of 12 HCGs) and 16 RSCGs, bringing the total number of compact groups in the full sample to 49 (see Table 3.1), and increasing the number of galaxies from 42 to 179. Of these 179 galaxies, 5 are saturated in one or more IRAC bands (HCG 16d, HCG56d, HCG 90d, HCG 92b, and RSCG 4b), and are not included in the fits or statistical analysis presented in this paper. Five of the 16 RSCGs $(21,44$, 66,67 , and 68) are known to be embedded in larger structures. We have undertaken

our analysis both with and without these groups, and in most cases, the results do not change. 


\section{Comparison Samples}

As in Walker et al. (2010), we compare our compact group sample with samples of galaxies in other environments. These samples are our approximation to a "field" sample - LVL+SINGS (Dale et al. 2009, 2007), interacting galaxies (Smith et al. 2007), and two samples from the Coma cluster - the center and the infall region (Jenkins et al. 2007). See Walker et al. (2010) for a discussion of these samples.

In order to compare samples of similar characteristics, we apply a luminosity cut to our samples, illustrated in Figure 3.1. We selected the lowest luminosity bin with

more than two compact group galaxies as the minimum luminosity for all of our samples. Thus our luminosity cut is $\log \left(L_{4.5}[\mathrm{erg} / \mathrm{s} / \mathrm{Hz}]\right)=27.5$. Figure 3.2 shows the Hubble types for our compact group sample against LVL+SINGS. As can be seen in this plot, the LVL+SINGS sample is dominated by late-type galaxies (which is to be expected for the field), while the compact group sample shows a bimodal distribution of morphological types, with a large number of early-type galaxies.

\subsubsection{Observations}

As this study utilizes archival data, the observational setups are heterogeneous. In addition to the Spitzer data, we also utilize data from the Two Micron All Sky Survey (Skrutskie et al. 2006) to plot IR spectral energy distributions (SEDs) to shorter $\lambda$.

\subsubsection{Photometry}

The IRAC images were first convolved to the $8 \mu \mathrm{m}$ PSF in order to ensure a common pixel scale and resolution, then photometry was performed using SURPHOT (Reines et al. 2008). The program determines apertures by finding a specified contour level in a reference image, then measuring the flux within the same aperture for each 
image. The apertures were determined using a combined image of all four IRAC bands (weighted by $\lambda^{-1}$ ), using a contour level of $1-2 \sigma$. Several background annuli were used, with inner radii ranging from $2-2.5 \times$ the radius of the aperture and outer radii ranging from $2.5-3 \times$ the radius of the aperture, using both the mode and resistant mean. Uncertainties were calculated from the standard deviation of the fluxes measured using different backgrounds. Extended source aperture corrections were made using the algorithms of T. Jarrett, and average corrections were $4 \%, 2 \%$, $9 \%$, and $14 \%$ at $3.6,4.5,5.8$, and $8.0 \mu \mathrm{m}$, respectively. There are slight differences from Johnson et al. (2007), likely due to variations in background measurements, though the new data are still consistent with the previous measurements. 


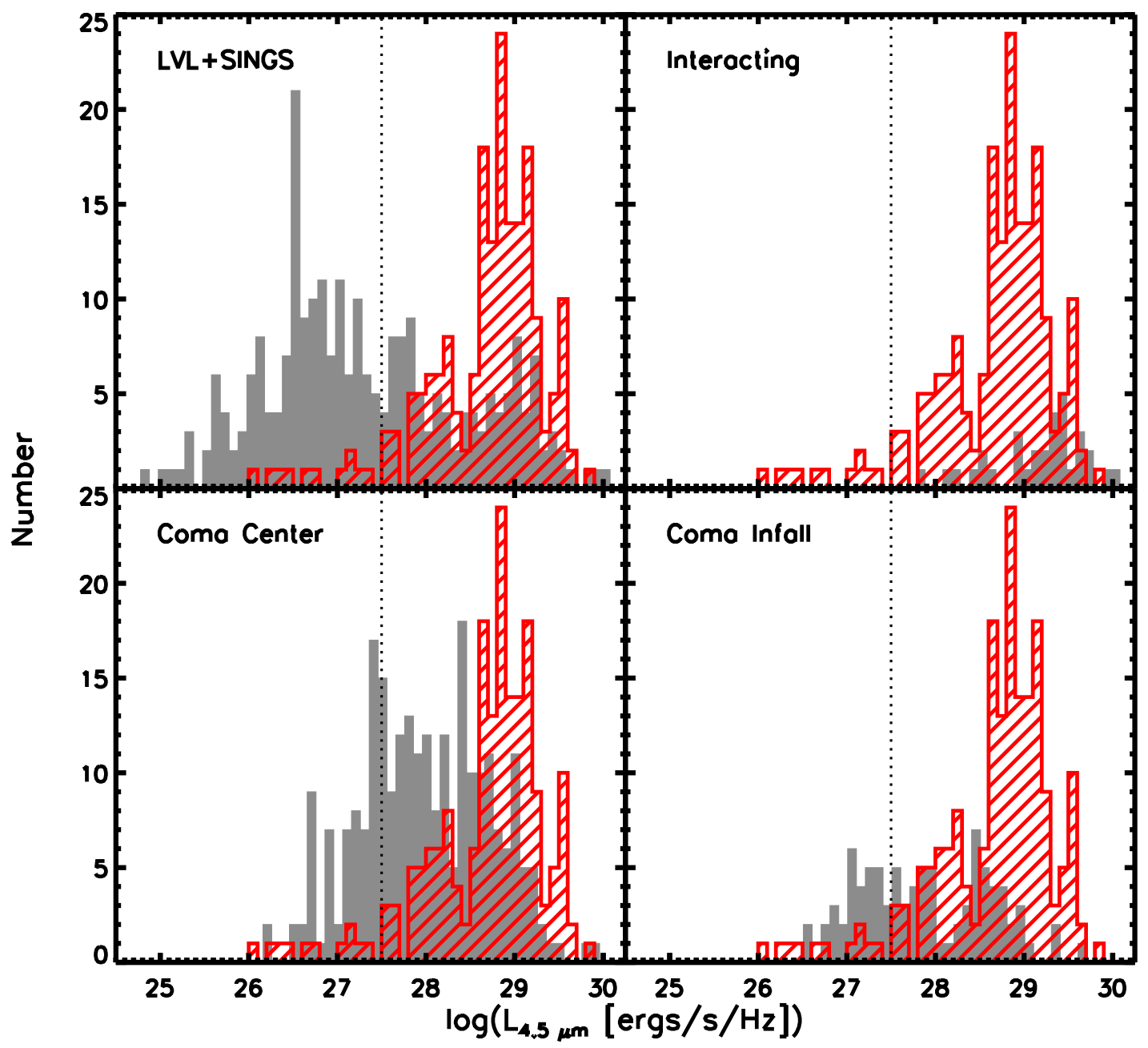

Fig. 3.1. - Histograms of $L_{4.5}$ for the compact group sample (striped) overlaid on the comparison samples (solid). The dotted vertical line indicates the luminosity cut. 


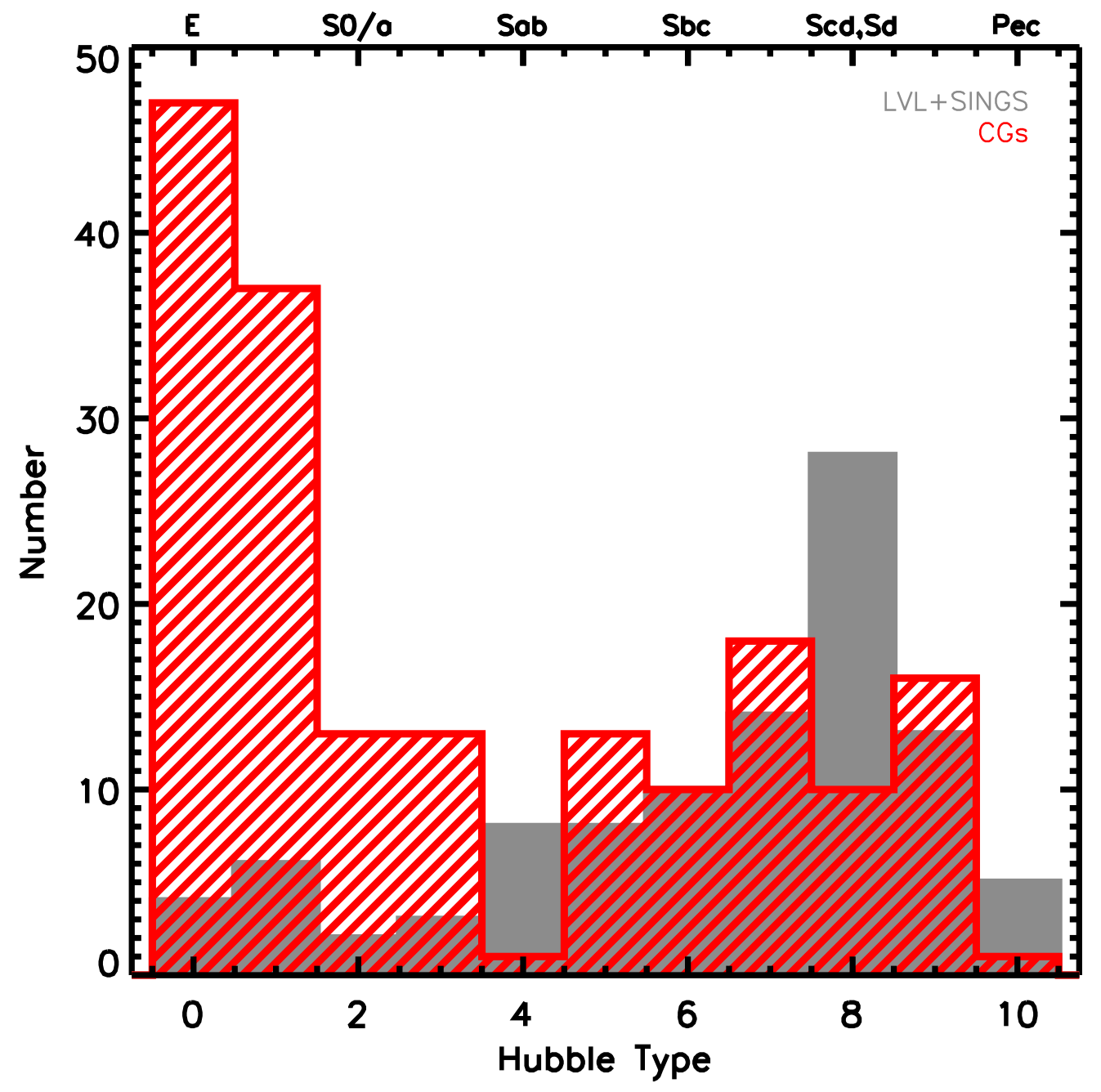

Fig. 3.2.- Distribution of morphological types (following Haynes \& Giovanelli 1984) for the compact group galaxies (striped) and LVL+SINGS galaxies (solid). As expected for a field sample, the LVL+SINGS galaxies are dominated by late-types, while the CG sample has a bimodal distribution. 
Table 3.1. CG Sample

\begin{tabular}{|c|c|c|c|c|}
\hline Group & $\begin{array}{c}\text { R.A. }{ }^{\mathrm{a}} \\
(\mathrm{J} 2000)\end{array}$ & $\begin{array}{c}\text { Dec }^{\mathrm{a}} \\
(\mathrm{J} 2000)\end{array}$ & $\begin{array}{c}\mathrm{D}^{\mathrm{a}} \\
(\mathrm{Mpc})\end{array}$ & $\operatorname{AOR}(\mathrm{s})$ \\
\hline H02 & $00^{\mathrm{h}} 31^{\mathrm{m}} 30^{\mathrm{s}} .0$ & $+08^{\circ} 25^{\prime} 52^{\prime \prime}$ & 136.6 & r11249152,r11252224 \\
\hline H04 & $00^{\mathrm{h}} 34^{\mathrm{m}} 15^{\mathrm{s}} .9$ & $-21^{\circ} 26^{\prime} 48^{\prime \prime}$ & 108.9 & r21926400 \\
\hline H07 & $00^{\mathrm{h}} 39^{\mathrm{m}} 23^{\mathrm{s}} .9$ & $+00^{\circ} 52^{\prime} 41^{\prime \prime}$ & 56.4 & r11249408,r11252480 \\
\hline H15 & $02^{\mathrm{h}} 07^{\mathrm{m}} 39^{\mathrm{s}} .0$ & $+02^{\circ} 08^{\prime} 18^{\prime \prime}$ & 90.8 & r26875648,r26880512 \\
\hline H16 & $02^{\mathrm{h}} 09^{\mathrm{m}} 31^{\mathrm{s}} \cdot 3$ & $-10^{\circ} 09^{\prime} 31^{\prime \prime}$ & 52.9 & r11249664,r11252736 \\
\hline H19 & $02^{\mathrm{h}} 42^{\mathrm{m}} 45^{\mathrm{s}} .1$ & $-12^{\circ} 24^{\prime} 43^{\prime \prime}$ & 57.3 & r11249920 \\
\hline $\mathrm{H} 22$ & $03^{\mathrm{h}} 03^{\mathrm{m}} 31^{\mathrm{s}} \cdot 3$ & $-15^{\circ} 40^{\prime} 32^{\prime \prime}$ & 36.1 & r11250176,r11252992 \\
\hline $\mathrm{H} 25$ & $03^{\mathrm{h}} 20^{\mathrm{m}} 43^{\mathrm{s}} .7$ & $-01^{\circ} 03^{\prime} 07^{\prime \prime}$ & 84.5 & r26785904,r26880768 \\
\hline H26 & $03^{\mathrm{h}} 21^{\mathrm{m}} 54^{\mathrm{s}} .2$ & $-13^{\circ} 38^{\prime} 45^{\prime \prime}$ & 126.9 & r23027712 \\
\hline H31 & $05^{\mathrm{h}} 01^{\mathrm{m}} 38^{\mathrm{s}} .3$ & $-04^{\circ} 15^{\prime} 25^{\prime \prime}$ & 54.6 & r11250432 \\
\hline H33 & $05^{\mathrm{h}} 10^{\mathrm{m}} 47^{\mathrm{s}} .9$ & $+18^{\circ} 02^{\prime} 05^{\prime \prime}$ & 105.1 & r23027968 \\
\hline H37 & $09^{\mathrm{h}} 13^{\mathrm{m}} 35^{\mathrm{s}} .6$ & $+30^{\circ} 00^{\prime} 51^{\prime \prime}$ & 95.5 & r23028224 \\
\hline H38 & $09^{\mathrm{h}} 27^{\mathrm{m}} 38^{\mathrm{s}} .8$ & $+12^{\circ} 16^{\prime} 51^{\prime \prime}$ & 124.3 & r23026432 \\
\hline H40 & $09^{\mathrm{h}} 38^{\mathrm{m}} 54^{\mathrm{s}} .5$ & $-04^{\circ} 51^{\prime} 07^{\prime \prime}$ & 96.9 & r23025920 \\
\hline H42 & $10^{\mathrm{h}} 00^{\mathrm{m}} 21^{\mathrm{s}} .8$ & $-19^{\circ} 38^{\prime} 57^{\prime \prime}$ & 53.3 & r11250688,r11253248 \\
\hline H47 & $10^{\mathrm{h}} 25^{\mathrm{m}} 48^{\mathrm{s}} .4$ & $+13^{\circ} 43^{\prime} 54^{\prime \prime}$ & 135.9 & r23026688 \\
\hline H48 & $10^{\mathrm{h}} 37^{\mathrm{m}} 45^{\mathrm{s}} \cdot 6$ & $-27^{\circ} 04^{\prime} 50^{\prime \prime}$ & 37.7 & r11250944,r11253504 \\
\hline H54 & $11^{\mathrm{h}} 29^{\mathrm{m}} 15.5$ & $+20^{\circ} 35^{\prime} 06^{\prime \prime}$ & 28.1 & r 23027456 \\
\hline H56 & $11^{\mathrm{h}} 32^{\mathrm{m}} 39^{\mathrm{s}} .6$ & $+52^{\circ} 56^{\prime} 25^{\prime \prime}$ & 116.0 & r23026944 \\
\hline H57 & $11^{\mathrm{h}} 37^{\mathrm{m}} 50^{\mathrm{s}} .5$ & $+21^{\circ} 59^{\prime} 06^{\prime \prime}$ & 131.7 & r23027200 \\
\hline H59 & $11^{\mathrm{h}} 48^{\mathrm{m}} 26^{\mathrm{s}} \cdot 6$ & $+12^{\circ} 42^{\prime} 40^{\prime \prime}$ & 54.1 & r11251200 \\
\hline H61 & $12^{\mathrm{h}} 12^{\mathrm{m}} 24^{\mathrm{s}} .9$ & $+29^{\circ} 11^{\prime} 21^{\prime \prime}$ & 52.1 & r11251456 \\
\hline H62 & $12^{\mathrm{h}} 53^{\mathrm{m}} 08^{\mathrm{s}} .1$ & $-09^{\circ} 13^{\prime} 27^{\prime \prime}$ & 54.9 & r11251712 \\
\hline H67 & $13^{\mathrm{h}} 49^{\mathrm{m}} 03^{\mathrm{s}} .5$ & $-07^{\circ} 12^{\prime} 20^{\prime \prime}$ & 109.9 & r26876160,r26881024 \\
\hline H68 & $13^{\mathrm{h}} 53^{\mathrm{m}} 40^{\mathrm{s}} .9$ & $+40^{\circ} 19^{\prime} 07^{\prime \prime}$ & 40.7 & r4483584,r27600128 \\
\hline & & & 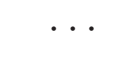 & r27600384,r27600640 \\
\hline
\end{tabular}




\subsection{Colorspace}

\subsubsection{Full Sample}

The IRAC colors of the full sample are presented in Figure 3.3, which shows that an underdensity of galaxies is persistent, though it has become more akin to a canyon than a gap. As the sample is now comprised of 179 galaxies, there are more galaxies with colors that place them within the canyon, though there is still a dearth of galaxies in this region relative to the number of galaxies with mid-IR-blue and midIR-red colors. The plot in Figure 3.3 shows the data with a linear fit, however a new result seen in this full sample of 49 compact groups is the curvature of the distribution in colorspace, which will be discussed in §3.6.4. To quantify this curvature, we fit a quadratic to the data:

$$
y=0.495+1.71 x-1.70 x^{2}
$$

also shown in Figure 3.3. Thus, we can undertake our analysis with respect to the line or the curve, both fit to the data.

For the statistical analysis, we considered the mid-IR colors in two ways: rotated so that the line shown in Figure 3.3 became the axis, and unwrapped so that the curve shown in Figure 3.3 became the axis. For both methods, the sample was shifted to have a mean value of zero. We have redefined the gap as the canyon using both sets of colors, as illustrated in Figure 3.4. In both plots, we define the canyon to be where the histogram is less than half its median value and $\mathrm{C}_{\mathrm{MIR}}$ is $<1.0$. The bounds of the canyon are indicated by the vertical dotted lines. The blue side of the canyon changes depending on whether we define it using the rotated or unwrapped color the canyon determined from the unwrapped data is slightly smaller. However, the results of our statistical analysis do not change. 
Table 3.1-Continued

\begin{tabular}{|c|c|c|c|c|}
\hline Group & $\begin{array}{c}\text { R.A. }^{\mathrm{a}} \\
(\mathrm{J} 2000)\end{array}$ & $\begin{array}{c}\text { Dec }^{\mathrm{a}} \\
(\mathrm{J} 2000)\end{array}$ & $\begin{array}{c}\mathrm{D}^{\mathrm{a}} \\
(\mathrm{Mpc})\end{array}$ & $\operatorname{AOR}(\mathrm{s})$ \\
\hline H71 & $14^{\mathrm{h}} 11^{\mathrm{m}} 04^{\mathrm{s}} .6$ & $+25^{\circ} 29^{\prime} 06^{\prime \prime}$ & 131.8 & r23028736 \\
\hline H79 & $15^{\mathrm{h}} 59^{\mathrm{m}} 11^{\mathrm{s}} .9$ & $+20^{\circ} 45^{\prime} 31^{\prime \prime}$ & 68.0 & r23028992 \\
\hline H90 & $22^{\mathrm{h}} 02^{\mathrm{m}} 06^{\mathrm{s}} .0$ & $-31^{\circ} 55^{\prime} 48^{\prime \prime}$ & 32.3 & r11251968,r11253760 \\
\hline H91 & $22^{\mathrm{h}} 09^{\mathrm{m}} 10^{\mathrm{s}} \cdot 4$ & $-27^{\circ} 47^{\prime} 45^{\prime \prime}$ & 99.1 & r26877184,r26881536 \\
\hline H92 & $22^{\mathrm{h}} 35^{\mathrm{m}} 57^{\mathrm{s}} .5$ & $+33^{\circ} 57^{\prime} 36^{\prime \prime}$ & 88.6 & r6011392 \\
\hline H96 & $23^{\mathrm{h}} 27^{\mathrm{m}} 58.3$ & $+08^{\circ} 46^{\prime} 27^{\prime \prime}$ & 118.5 & r12297728 \\
\hline H97 & $23^{\mathrm{h}} 47^{\mathrm{m}} 22.9$ & $-02^{\circ} 19^{\prime} 34^{\prime \prime}$ & 88.3 & r26877440,r26881792 \\
\hline H100 & $00^{\mathrm{h}} 01^{\mathrm{m}} 20.8$ & $+13^{\circ} 07^{\prime} 57^{\prime \prime}$ & 71.9 & r26877696 \\
\hline R04 & $00^{\mathrm{h}} 42^{\mathrm{m}} 49.5$ & $-23^{\circ} 33^{\prime} 11^{\prime \prime}$ & 89.0 & r12342784 \\
\hline $\mathrm{R} 06$ & $01^{\mathrm{h}} 16^{\mathrm{m}} 12^{\mathrm{s}} .4$ & $+46^{\circ} 44^{\prime} 20^{\prime \prime}$ & 69.1 & r26227712 \\
\hline $\mathrm{R} 15$ & $01^{\mathrm{h}} 52^{\mathrm{m}} 41^{\mathrm{s}} .4$ & $+36^{\circ} 08^{\prime} 46^{\prime \prime}$ & 64.3 & r18646272 \\
\hline $\mathrm{R} 17$ & $01^{\mathrm{h}} 56^{\mathrm{m}} 21^{\mathrm{s}} .8$ & $+05^{\circ} 38^{\prime} 37^{\prime \prime}$ & 75.5 & r18030336 \\
\hline $\mathrm{R} 21^{\dagger}$ & $03^{\mathrm{h}} 19^{\mathrm{m}} 36^{\mathrm{s}} .6$ & $+41^{\circ} 33^{\prime} 39^{\prime \prime}$ & 68.7 & r10483456 \\
\hline R31 & $09^{\mathrm{h}} 17^{\mathrm{m}} 26.5$ & $+41^{\circ} 57^{\prime} 18^{\prime \prime}$ & 29.5 & r23113472,r23113728 \\
\hline R32 & $09^{\mathrm{h}} 19^{\mathrm{m}} 50.8$ & $+33^{\circ} 46^{\prime} 17^{\prime \prime}$ & 96.3 & r4438784 \\
\hline $\mathrm{R} 34$ & $09^{\mathrm{h}} 43^{\mathrm{m}} 12^{\mathrm{s}} .6$ & $+31^{\circ} 54^{\prime} 44^{\prime \prime}$ & 25.7 & r4439552,r16300032 \\
\hline R38 & $10^{\mathrm{h}} 51^{\mathrm{m}} 46^{\mathrm{s}} .7$ & $+32^{\circ} 51^{\prime} 31^{\prime \prime}$ & 27.2 & r16304640 \\
\hline $\mathrm{R} 42$ & $11^{\mathrm{h}} 36^{\mathrm{m}} 51^{\mathrm{s}} \cdot 3$ & $+19^{\circ} 59^{\prime} 19^{\prime \prime}$ & 93.5 & r25498880 \\
\hline $\mathrm{R} 44^{\dagger}$ & $11^{\mathrm{h}} 44^{\mathrm{m}} 00^{\mathrm{s}} \cdot 6$ & $+19^{\circ} 56^{\prime} 44^{\prime \prime}$ & 93.6 & r3858688 \\
\hline $\mathrm{R} 64$ & $12^{\mathrm{h}} 41^{\mathrm{m}} 33.2$ & $+26^{\circ} 03^{\prime} 56^{\prime \prime}$ & 73.7 & r10532608 \\
\hline $\mathrm{R} 66^{\dagger}$ & $12^{\mathrm{h}} 43^{\mathrm{m}} 17^{\mathrm{s}} .9$ & $+13^{\circ} 11^{\prime} 47^{\prime \prime}$ & 13.9 & r4477440,r18288896 \\
\hline $\mathrm{R} 67^{\dagger}$ & $12^{\mathrm{h}} 59^{\mathrm{m}} 32^{\mathrm{s}} .8$ & $+27^{\circ} 57^{\prime} 27^{\prime \prime}$ & 106.4 & r3859456 \\
\hline $\mathrm{R} 68^{\dagger}$ & $13^{\mathrm{h}} 00^{\mathrm{m}} 10^{\mathrm{s}} .7$ & $+27^{\circ} 58^{\prime} 17^{\prime \prime}$ & 98.3 & r3859712,r3859968 \\
\hline $\mathrm{R} 86$ & $23^{\mathrm{h}} 38^{\mathrm{m}} 34^{\mathrm{s}} .4$ & $+27^{\circ} 01^{\prime} 24^{\prime \prime}$ & 117.6 & r10926592 \\
\hline
\end{tabular}

${ }^{a}$ Group RA, Dec and distance (corrected for Virgo+GA+Shapley) taken from NED. The cosmology used was $H_{0}=73 \mathrm{~km} \mathrm{~s}^{-1} \mathrm{Mpc}^{-1}$, $\Omega_{\text {matter }}=0.27$, and $\Omega_{\text {vacuum }}=0.73$.

${ }^{\dagger} \mathrm{RSCGs}$ known to be embedded in larger structures. 


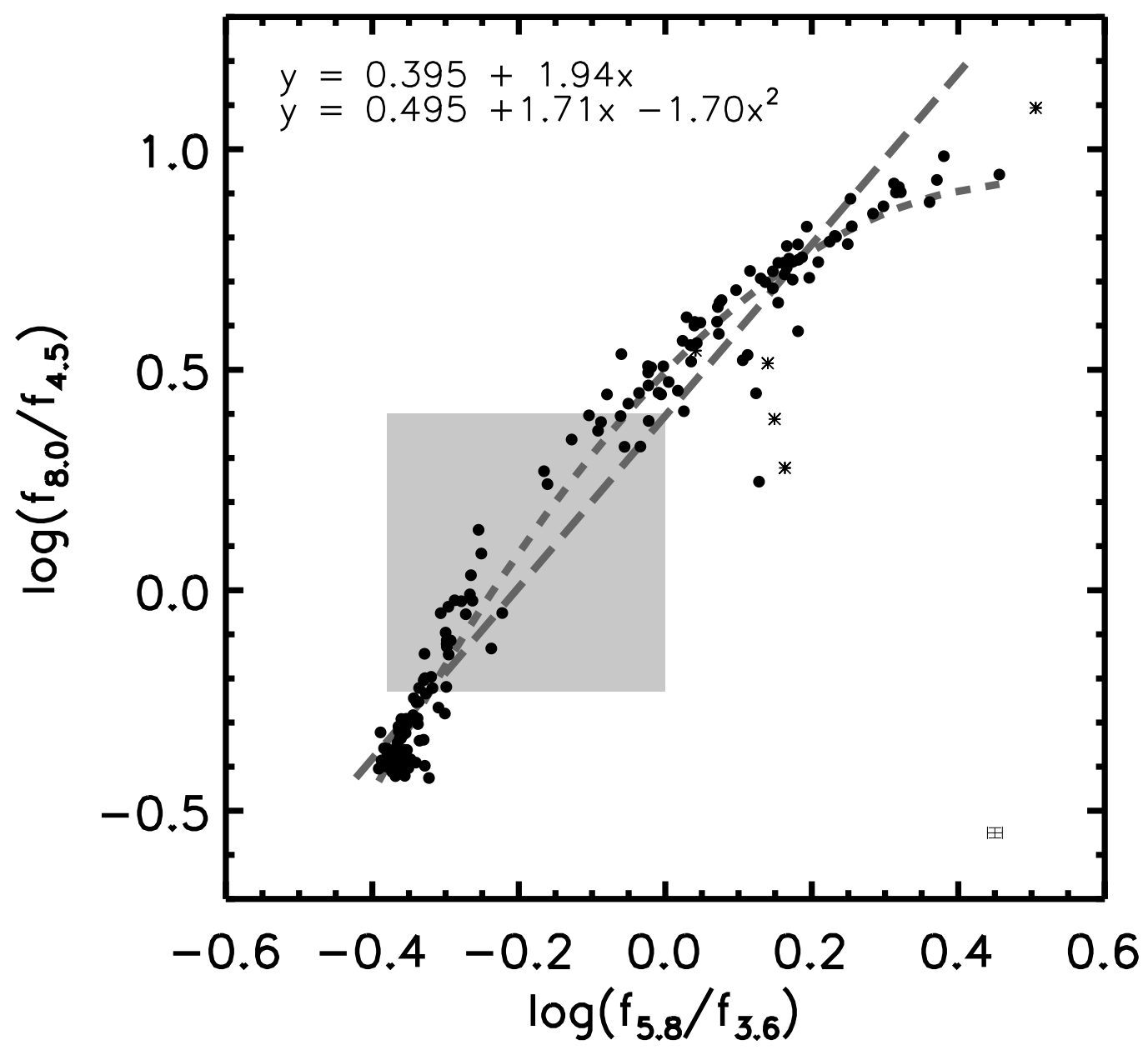

Fig. 3.3. - The colorspace distribution of the 179 compact group galaxies comprising the full sample; the shaded box highlights the location of the gap in the original sample (Walker et al. 2010). The asterisks indicate galaxies that are mildly saturated in one or more IRAC channels, these 5 galaxies are not included in further analysis. We performed two different fits to the data, shown above. 

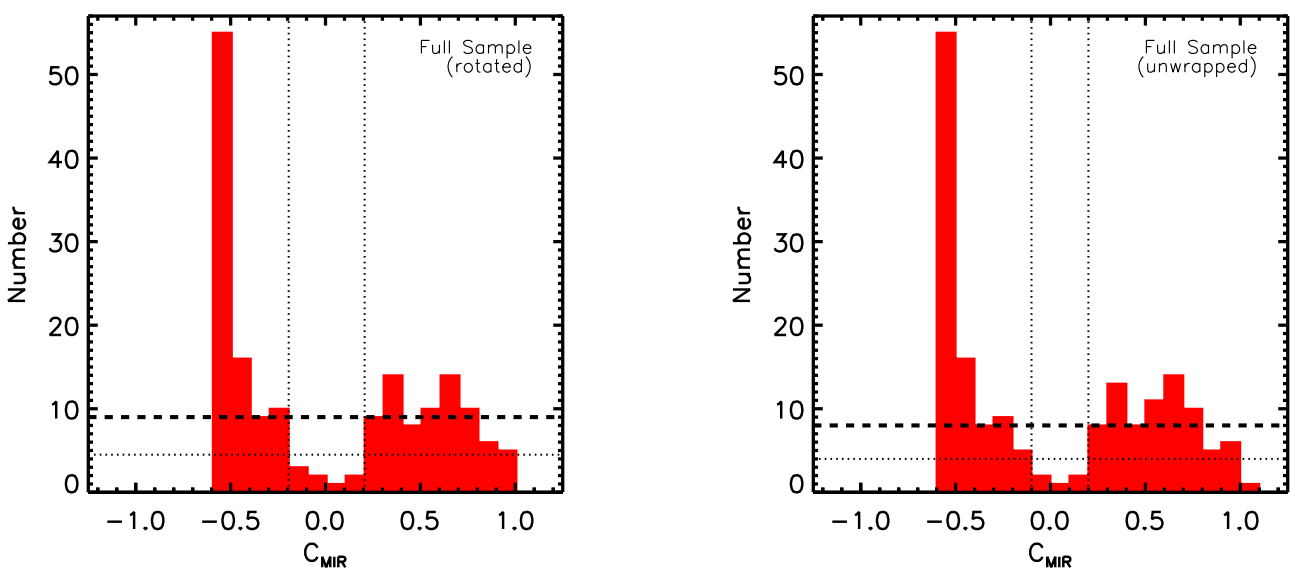

Fig. 3.4. - Histograms of the left: rotated colorspace distribution and right: unwrapped colorspace distribution as described in §3.3.1. In both plots, the dashed line indicates the median, the horizontal dotted line is half that value. The vertical dotted lines indicate the boundaries of the canyon, defined to be where the distribution is less than half the median value with an mid-IR color $<1.0$. In the rotated sample, $5 \%$ of the galaxies fall in the canyon, while for the unwrapped sample, $3 \%$ of galaxies fall in the canyon.

Table 3.2. Results of Statistical Analysis

\begin{tabular}{cccccc}
\hline \hline Compact Groups vs & $\mathrm{N}$ & $\mathrm{N}_{\mathrm{CGs}}$ & $\mathrm{D}$ & $\alpha$ & Consistent? \\
\hline Uniform Distribution & $\ldots$ & 174 & 0.30 & $2.0 \times 10^{-13}$ & reject \\
LVL+SINGS & 93 & 174 & 0.47 & $3.8 \times 10^{-12}$ & reject \\
Interacting & 31 & 78 & 0.41 & $1.6 \times 10^{-3}$ & reject \\
Coma Center & 114 & 173 & 0.36 & $6.0 \times 10^{-8}$ & reject \\
Coma Infall & 47 & 173 & 0.18 & 0.16 & do not reject \\
\hline
\end{tabular}

Note. - Results of the KS test for the compact group distribution against a model and the comparison samples, unwrapped using the quadratic fit to the compact group distribution. $D$ is the maximum deviation (possible range: $0-1$ ) between the two CDFs, while $\alpha$ gives the probability that the two samples were drawn from the same parent distribution (which depends on the number of galaxies in the two samples, $N$, and $\left.N_{\mathrm{CGs}}\right)$. Based on $\alpha$, we have determined whether the two samples are consistent. 
The plots in Figure 3.5 show the results of the Kolmogorov-Smirnov (KS) test comparing the rotated and unwrapped color distributions with a model of a uniform distribution, where $D$ is the maximum difference between the sample and the model, and $\alpha$ gives the probability that the model matches the data. While we do not expect any particular sample to have a uniform distribution, using a model of a uniform distribution as a baseline facilitates comparisons between environments. The grey region indicates the canyon, which is clearly manifested in this plot as the flat portion of the cumulative distribution function ( $\mathrm{CDF})$. The canyon is less extended in this larger data set than the gap seen in the original sample, but it is still present, and $\alpha$ is small enough that we can conclusively state that the compact group sample is not drawn from a uniform distribution in colorspace. The two methods of unraveling the mid-IR colors yield the same result in the KS test. Further analysis uses the unwrapped colors, because the canyon galaxies as defined by the unwrapped sample are more cleanly defined in colorspace (shown in Figure 3.6). Excluding the five RSCGs that are embedded in larger structures $(21,44,66,67$, and 68) increases $\alpha$ by an order of magnitude, but does not change the result.

\subsubsection{Subsamples}

To investigate how the compact group environment affects galaxy evolution, we investigated the colorspace distribution of various subsamples. If a clear trend with physical properties were found, it would provide a clear picture of how environment affects galaxy evolution in compact groups. 

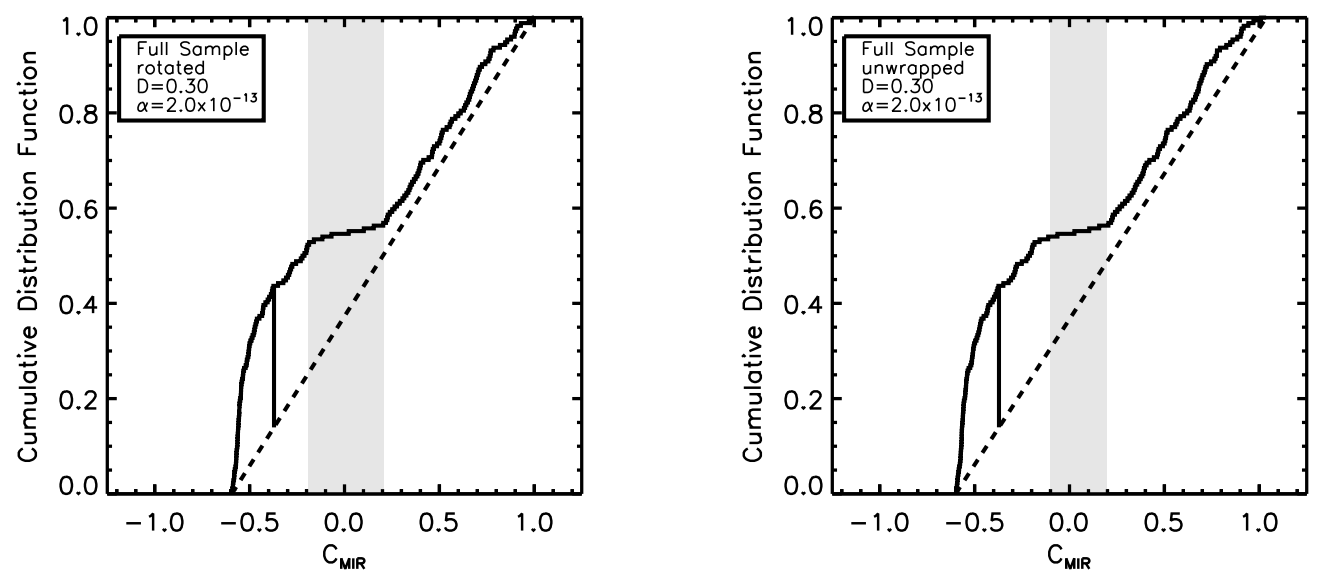

Fig. 3.5. - KS test for the left: rotated colorspace distribution and right: unwrapped colorspace distribution of the full sample against a model of uniform distribution. The maximum deviation $D$ of the sample from the model is indicated by the vertical line. This large value of $D$ means a low probability that the compact groups are drawn from a uniform distribution, $2 \times 10^{-11} \%$.
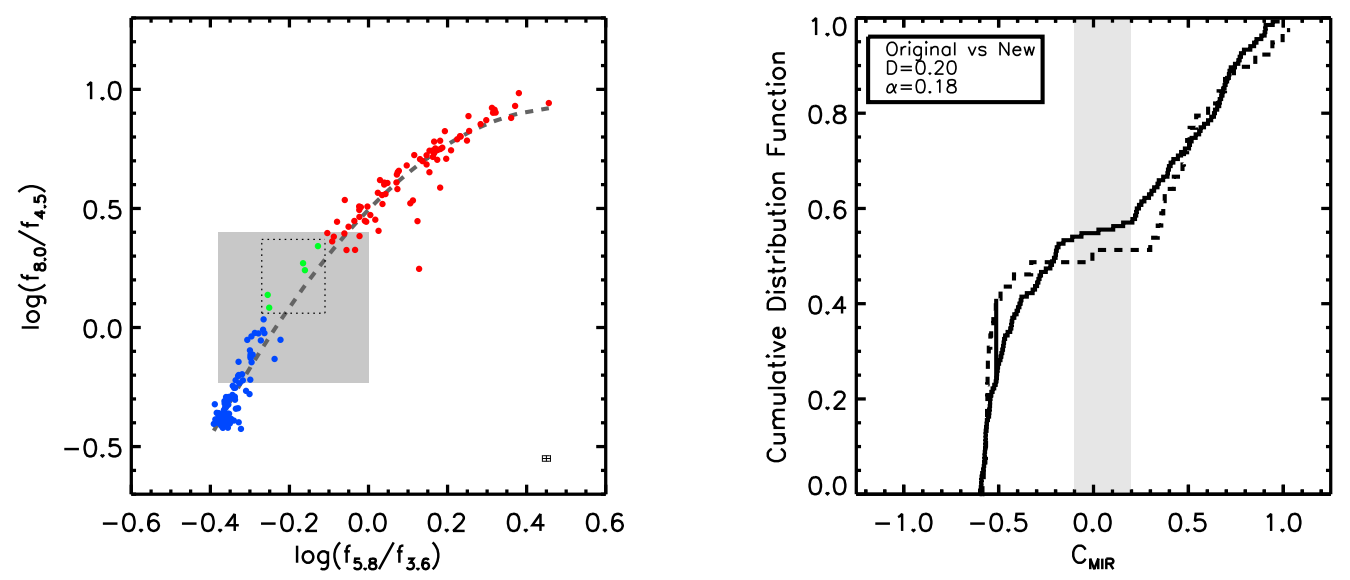

Fig. 3.6. - Left: The colorspace distribution of the compact group galaxies, with symbol color illustrating whether a galaxy is blueward (blue), in (green), or redward (red) of the gap. Right: KS test comparing the original sample of 12 HCGs (dashed line) with the new sample of 37 CGs (solid line). These two samples are very similar, with a fairly high probability of being drawn from the same parent distribution, $18 \%$. 


\section{Original vs New}

To insure that the properties of the expanded sample are consistent with the original sample, we compare it with the original sample. The CDFs of the new and original sample are shown on the right in Figure 3.6, which illustrates that the new sample is consistent with the original sample. The difference in the gap seen in the original sample and the canyon defined by the full sample (highlighted by the shaded region) is clearly illustrated by the different ranges of the flat portion of the CDF - the canyon is narrower and slightly less pronounced than the original gap.

\section{HCGs vs RSCGs}

To determine whether the different selection criteria of the HCG and RSCG catalogs find groups with different properties, we compare groups from the two catalogs. The colorspace distribution of the HCG galaxies and RSCG galaxies are shown in Figure 3.7. We see that the HCGs and RSCGs occupy colorspace very similarly.

\section{Separated by Physical Properties}

As compact group galaxies occupy colorspace differently than galaxies in other environments (shown in Table 3.2 and §3.3.3, as well as Walker et al. 2010), we expect to see a trend with physical properties of compact groups, especially the properties which differentiate them from other environments. Thus we binned the groups by projected physical diameter and projected physical number density and examined colorspace as a function of these properties, as shown in Figure 3.8. Both of these properties could be an indicator of how frequently or intensely interactions occur, which could plausibly affect the triggering of star formation and/or transformation from activity to quiesence. However, as these figures show, there does not seem to be 
any trend with these properties. Further investigation of the CDFs of the subsamples reveals that the shape of the subsample's CDF does not correlate with either of these properties. This will be discussed further in §3.6.2.

\subsubsection{Comparison Samples}

The colorspace distributions of our comparison samples are shown in Figure 3.9. As this figure illustrates, the LVL+SINGS sample spans almost the exact same region of colorspace as the compact group sample, though there is no evidence for a canyon. The interacting sample does not occupy the blue region of colorspace, indicating that this sample does not contain any galaxies dominated by stellar emission. In contrast, galaxies in the center of the Coma cluster predominantly fall in the blue region of colorspace, with only a few galaxies whose colors indicate activity. Like the Coma center, the infall region of the Coma cluster shows a concentration of galaxies with normal stellar colors, but also contains galaxies with colors indicative of activity and exhibits an underdensity of points in the same region as the canyon in the compact group sample. We performed two-distribution KS tests comparing these samples with the compact group sample; the results are given in Table 3.2.

It is important to consider the colorspace distributions of the samples in the context of the morphologies of their galaxies, given in Figure 3.2 for the compact group and field samples. As discussed in Walker et al. (2010), untangling the effects of morphology on mid-IR color is non-trivial. To some extent, morphology and mid-IR color are expected to track each other; e.g., late-type spirals are typically starforming and therefore expected to have red mid-IR colors. Therefore, it could be the case that the different morphological types that dominate each of the comparison samples is driving the KS test results. For example, the interacting galaxy sample has no 

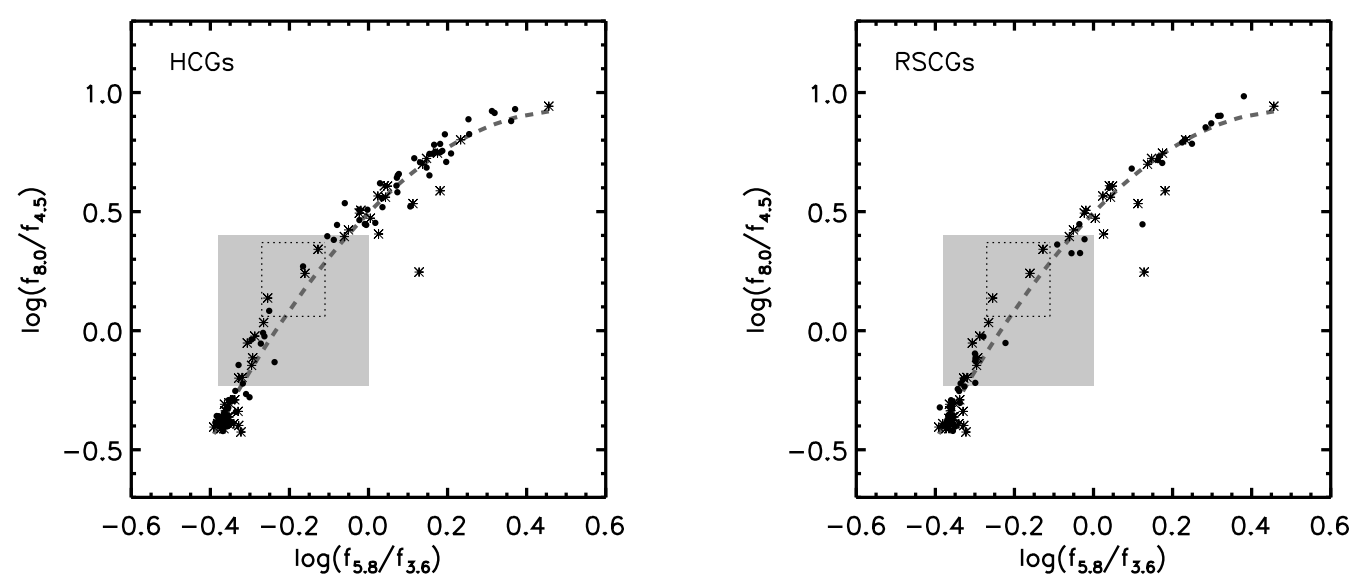

Fig. 3.7. - Left: The colorspace distribution of left: the 124 HCG galaxies and right: the 50 RSCG galaxies.
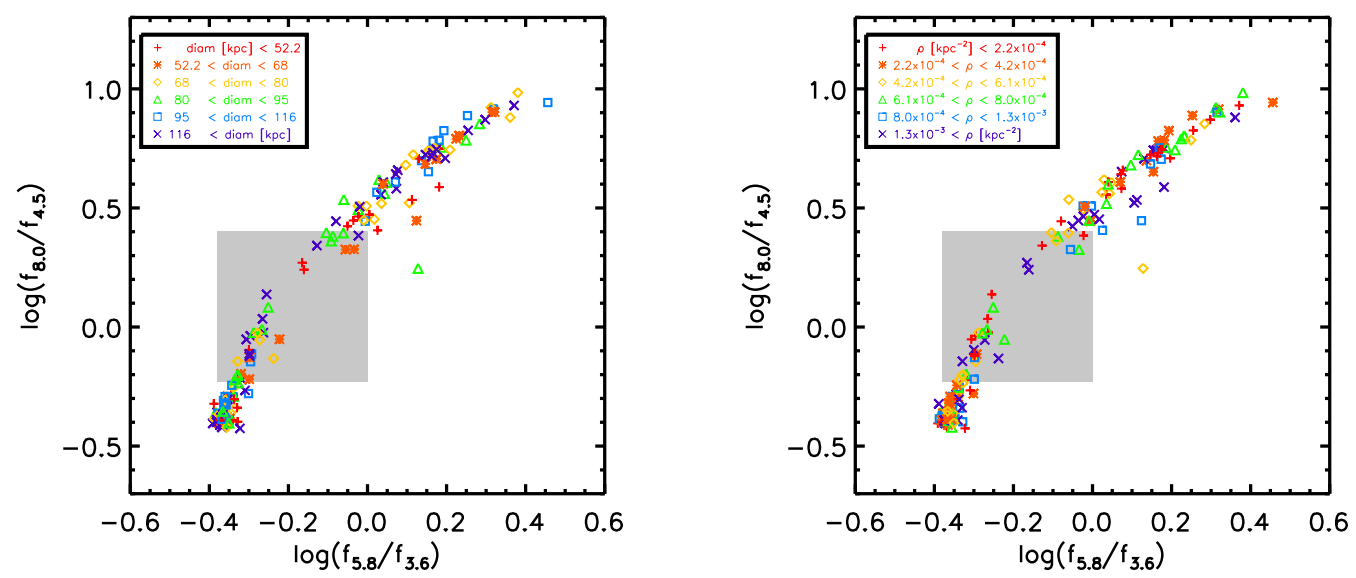

Fig. 3.8. - The colorspace distribution of the full sample, broken into subsamples based on left: projected physical diameter and right: projected physical density. Neither of these plots show a trend, indicating that these parameters do not play a key role in determining a galaxy's location in colorspace within the range represented by the compact group sample. 
E/S0 galaxies by selection, and the morphology-density relation means that primarily quiescent E/S0s are found in the Coma Core sample. Unlike these two samples, both the compact group and LVL+SINGS samples span the range of morphological types. While mid-IR color tracks morphology quite well in the compact group sample, this is not the case for the LVL+SINGS galaxies (Walker et al. 2010). Thus, while the dearths seen in both colorspace and morphological distribution for the compact group sample may be caused by the same evolutionary process, the causal relationship is unclear.

\subsection{Color-Magnitude Diagrams}

Galaxy evolution is tied to galaxy size and buildup. If the compact group galaxies show a trend with mass or activity, this would be revealed in a color-magnitude diagram (CMD). Comparison of the CG CMD with LVL+SINGS and Coma Infall (shown in Figures 3.10, 3.11 and 3.12) provide insight into galaxy properties. All three samples show a trend for mid-IR-red galaxies to be more luminous. This is not surprising, as we would expect that galaxies with activity would have more warm dust and PAH emission, thus increasing their $L_{8.0}$. One notable feature is the dearth of intermediate-luminosity, mid-IR-blue galaxies in the field sample, though this could be a selection effect, specifically that the SINGS sample was chosen to include "interesting" galaxies, thus mid-luminosity, mid-IR-blue galaxies may not have been included. 


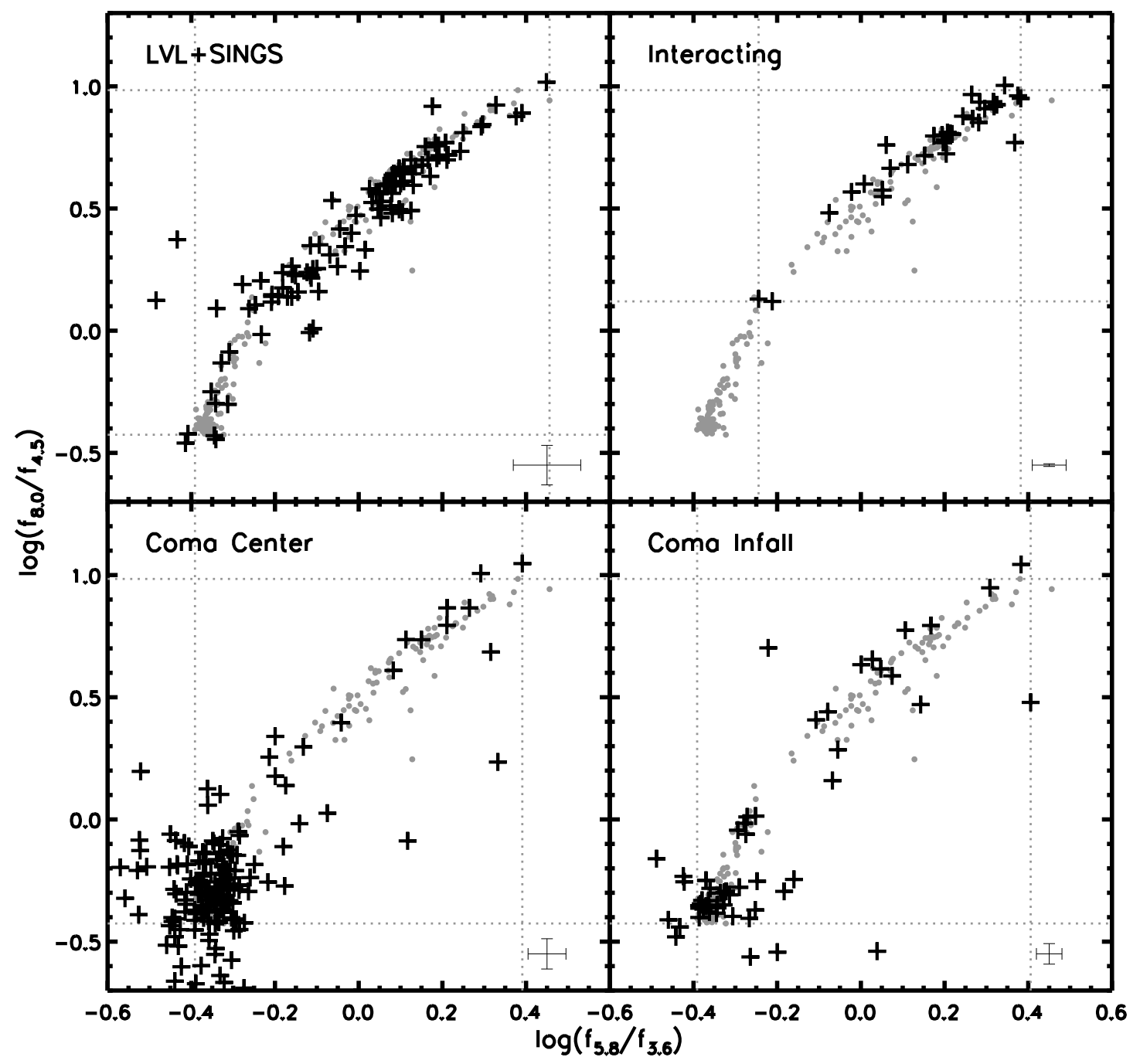

Fig. 3.9.- Colorspace distribution of the comparison samples (black plus signs) overlaid on the compact group sample (grey dots). 

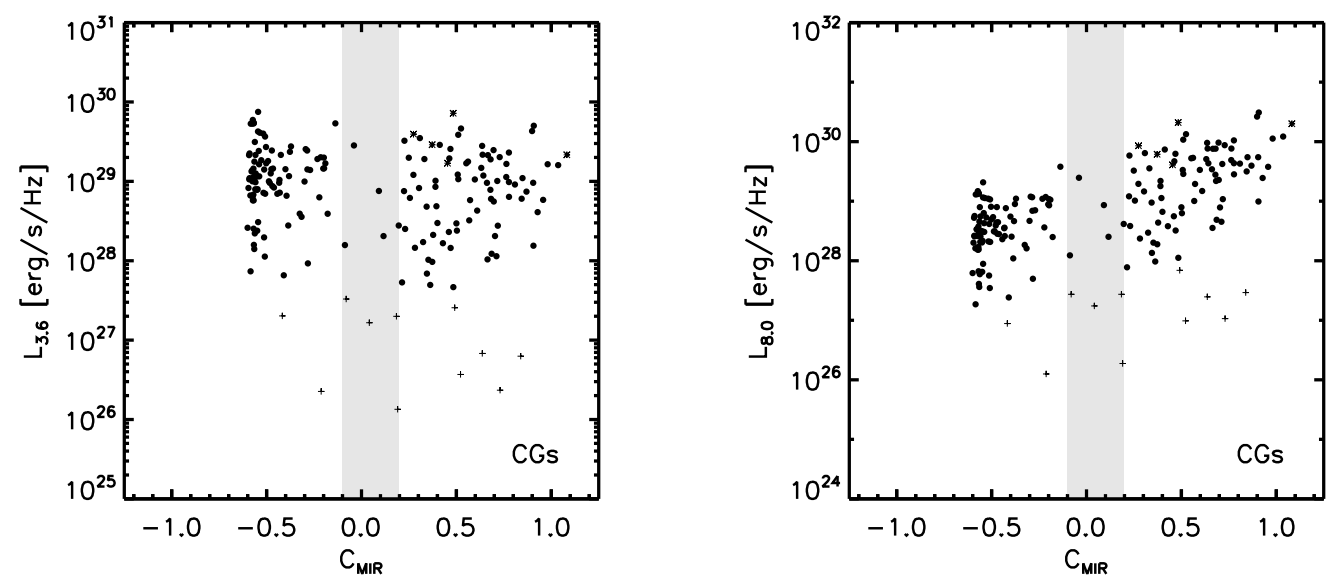

Fig. 3.10.- $L_{3.6}$ (left) and $L_{8.0}$ (right) color-magnitude diagrams for compact group galaxies. The plus signs represent galaxies below the luminosity cut (as discussed in $\S 3.2 .1$ and Figure 3.1), the asterisks indicate saturated galaxies. The shaded region indicates the canyon in IRAC colorspace.
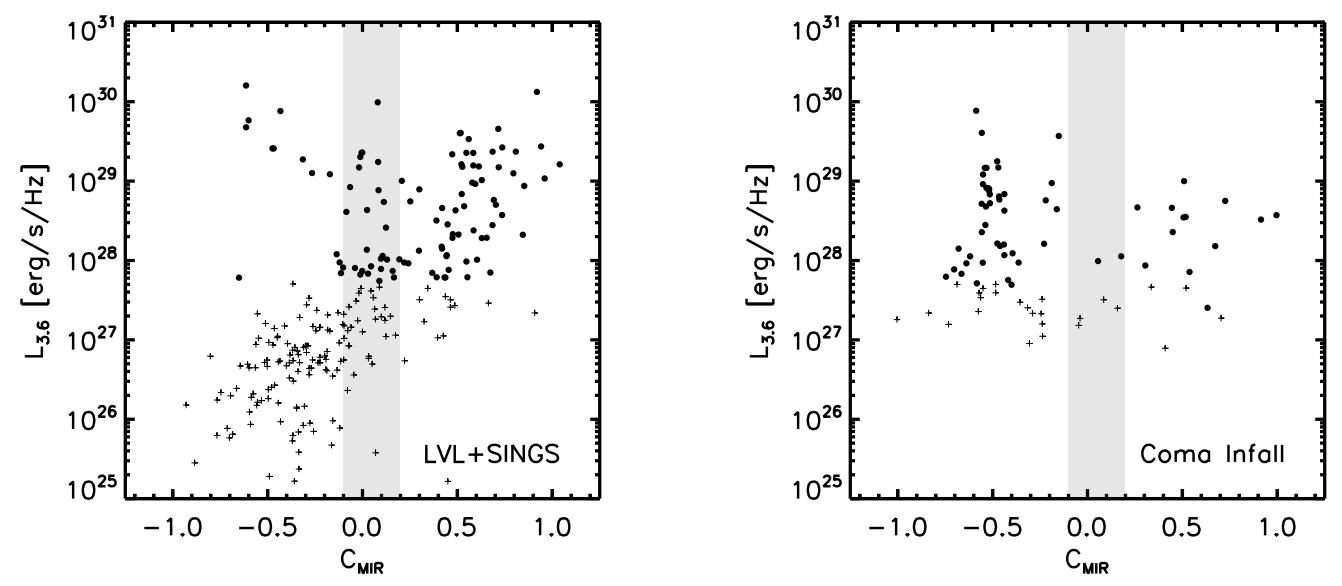

Fig. 3.11. - $L_{3.6}$ color-magnitude diagrams of galaxies in left: the field sample of LVL+SINGS and right: the infall region of the Coma cluster. The plus signs represent galaxies below the luminosity cut (as discussed in §3.2.1 and Figure 3.1). The shaded region indicates the canyon in IRAC colorspace. 

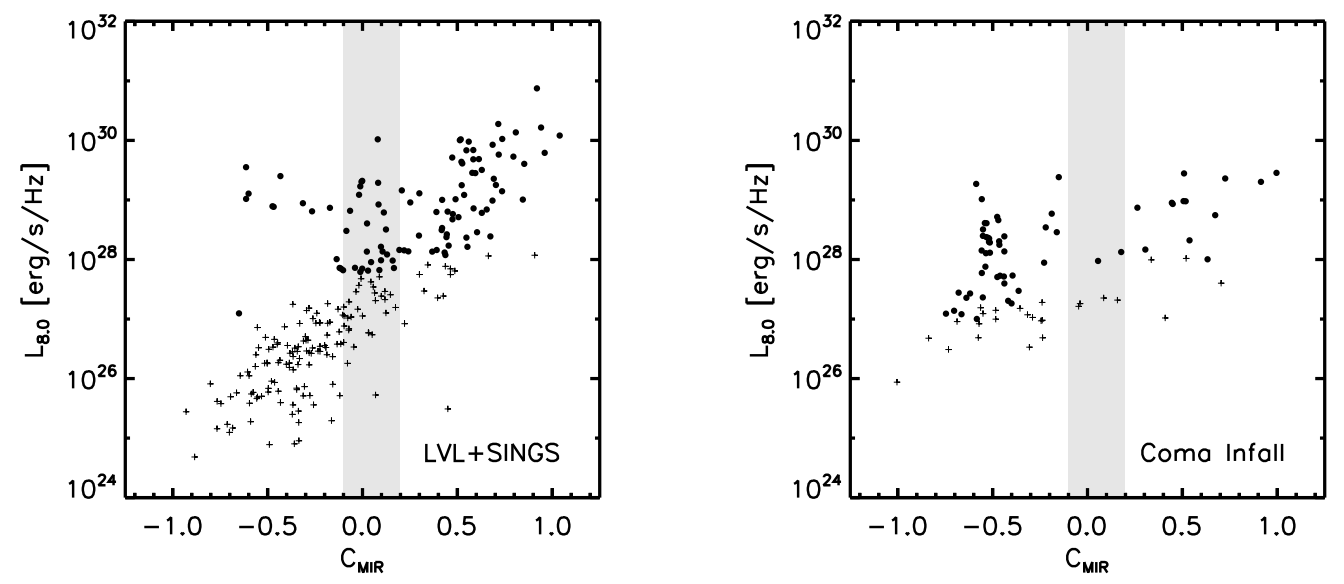

Fig. 3.12. - $L_{8.0}$ color-magnitude diagrams of galaxies in left: the field sample of LVL+SINGS and right: the infall region of the Coma cluster. The plus signs represent galaxies below the luminosity cut (as discussed in $§ 3.2 .1$ and Figure 3.1). The shaded region indicates the canyon in IRAC colorspace.
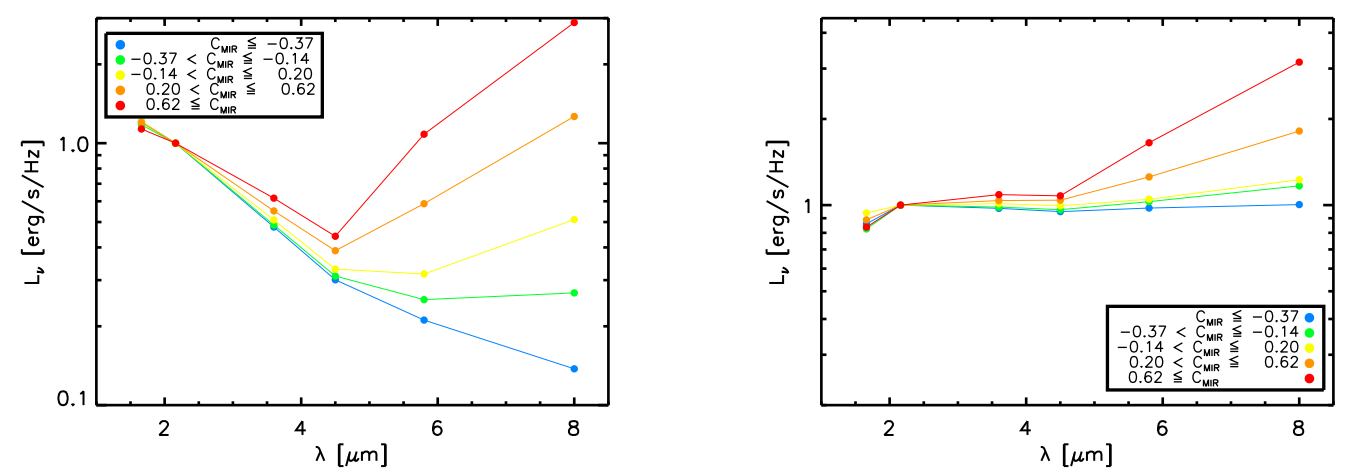

Fig. 3.13.- Binned SEDs of compact group galaxies, normalized to K-band, as a function of location in mid-IR colorspace. The yellow SED is comprised of canyon galaxies. The plot on the left shows SEDs with contributions from both stars and dust. The plot on the right shows stellar-subtracted SEDs, allowing us to study the dust. 


\subsection{Spectral Energy Distributions}

In order to investigate the relative contributions of amount and temperature of dust in compact group galaxies as a function of colorspace, we have binned SEDs of individual galaxies as a function of location in colorspace, shown on the left in Figure 3.13. Since we are interested in the ISM, we extracted the contribution from stellar light by scaling a $5000 \mathrm{~K}$ blackbody to the K-band emission. If the dust properties (e.g. temperature, composition, excitation) are the same in galaxies across colorspace, and the variation in mid-IR color is simply due to different amounts of dust and PAH emission, we expect the slope of the stellar-subtracted SEDs to be the same regardless of location in colorspace. If the different mid-IR colors are in fact dominated by varying dust properties, we expect the slope of the stellar-subtracted SEDs to change as a function of mid-IR color. As can be seen on the right in Figure 3.13 the stellar-subtracted SEDs do have clearly different slopes as a function of mid-IR color, which means that the range of mid-IR colors we see in the CG sample is not simply due to varying amounts of dust between galaxies. An analysis of the LVL+SINGS SEDs yields a similar result. Thus, the trend in IRAC colorspace can be attributed to the properties of dust in the galaxies, and the canyon reflects a range of moderate dust properties.

\subsection{Discussion}

We have compiled a full sample of 49 compact groups comprised of 33 HCGs and 16 RSCGs. We see that the deficit (previously identified as a gap, see §3.1) between galaxies with colors consistent with normal stellar emission (mid-IR blue) and galaxies with colors indicative of activity (mid-IR red) is persistent in the larger sample. We investigate physical trends as a function of colorspace and look at the structure of the 
colorspace distribution. We also compare the compact group colorspace distribution with samples of galaxies in other environments. We consider the SEDs of the galaxies to assess whether dust quantity or temperature changes as a function of colorspace.

\subsubsection{The Canyon}

The gap seen in the original sample of 12 HCGs (Johnson et al. 2007; Walker et al. 2010) needs to be redefined in terms of the full sample presented here. While there is still an underdensity between galaxies whose colors are consistent with normal stellar populations and galaxies whose colors are indicative of activity, it is no longer the clear-cut gap seen originally. Now with better statistics, it is sparsely populated and

thus more of a canyon. As shown in Figure 3.4, we quantified the location of the canyon via a histogram of the colorspace distribution. As the full sample has $\sim 4 \times$ more galaxies than the original sample, there is naturally a larger absolute number of galaxies in the canyon, though the relative number of galaxies are comparable $(2.4 \%$ of galaxies from the original sample, $2.8 \%$ of galaxies from the expanded sample). The larger number of galaxies in this region will allow us to learn about properties of galaxies that exhibit these intermediate colors.

\subsubsection{Impact of Subsamples}

The larger number of galaxies available in the full sample allows us to look for trends in colorspace. Comparing the HCGs with the RSCGs shows that they are consistent with being drawn from the same parent distribution. Breaking the full sample into subsamples based on projected physical diameter or density does not reveal any trends in colorspace. This is a puzzling result - we hypothesize that compact groups are quantitatively different from other samples in mid-IR colorspace due to our analysis 
of the compact group and comparison samples, yet we do not see any trends with these properties within the compact group sample. It appears that projected physical diameter and density are not the dominant properties in determining the colorspace location of compact group galaxies over the parameter ranges of this sample. It may simply be that the compact group environment is pre-selected to be compact and dense, and thus does not have a large enough variation in group diameters or densities to reveal trends in colorspace. However, Johnson et al. (2007) found that the original sample exhibits a trend in colorspace with group H I richness, so this seems to be the crucial property in colorspace distribution. Regardless of a group's diameter or density, star formation relies on the presence of cold gas.

\subsubsection{Environment}

In agreement with previous work (Walker et al. 2010), the colorspace distribution of the compact group sample is statstically different from a control sample of "field" galaxies, a sample of interacting galaxies, and galaxies from the center of the Coma cluster. However, we cannot rule out the hypothesis that the compact group sample is drawn from the same parent distribution as the sample from the infall region of the Coma cluster. This supports the hypothesis that there is something special about this environment - one of high galaxy density where the neutral gas has not typically been fully preprocessed (meaning stripped, ionized, expelled, or otherwise modified

through a galaxy interaction process). Cluver et al. (2013) found that galaxies in or near the canyon show anamolous $\mathrm{H}_{2}$ excitation, similar to that seen in the shock in Stephan's Quintet (HCG92; Cluver et al. 2010). This could be related to a rapid end to star formation in compact group galaxies, leading to the canyon observed in IRAC colorspace. 


\subsubsection{SEDs}

The full sample revealed a curvature in colorspace not apparent in the original sample that was sparsely populated. The changing shape of the dust-subtracted SEDs as a function of mid-IR color indicate that the colors are not dominated solely by varying amounts of dust within the galaxies. Rather, the dust temperature distribution and/or PAH contributions change as a function of mid-IR color. We are unable to determine dust temperatures for these galaxies because the IRAC bands contain significant PAH features so we cannot disentangle the PAH emission from the dust temperature, and since the SEDs are not turning over, we do not cover the peak of the emission. We have looked at IRS spectra of compact group galaxies in different regions of IRAC colorspace, and found that the spectra of the bluest galaxies decrease with wavelength and show no PAH features. The PAH features then appear fairly quickly as you consider redder galaxies. They seem to be fully formed by the canyon region, and do not change significantly as you look redward. The primary change redward of the canyon seems to be the slope of the spectra (Walker et al. in prep). This suggests that galaxies undergoing activity have different dust compositions than quiescent galaxies.

\subsubsection{Conclusions}

We have determined that the distribution of compact group galaxies in mid-IR colorspace still shows an underdensity in the canyon region of colorspace. This distribution is most similar to that of the Coma infall region, implying a similarity in environment. The distribution of colors is caused by varying dust temperatures and $\mathrm{PAH}$ emission rather than varying amounts of dust. However, there are still many unexplained properties of this expanded sample. 
Chapter 4

The Optical Green Valley vs

Mid-IR Canyon in Compact

Groups 


\section{Abstract}

Compact groups of galaxies provide conditions similar to those experienced by galaxies in the earlier universe. Recent work on compact groups has led to the discovery of a dearth of mid-infrared transition galaxies (MIRTGs) in IRAC (3.6-8.0 $\mu \mathrm{m})$ color space (Johnson et al. 2007; Walker et al. 2012) as well as at intermediate specific star formation rates (Tzanavaris et al. 2010). However, we find that in compact groups these mid-infrared (mid-IR) transition galaxies in the mid-infrared dearth have already transitioned to the optical $([\mathrm{g}-\mathrm{r}])$ red sequence. We investigate the optical color-magnitude diagram (CMD) of 99 compact groups containing 348 galaxies and compare the optical CMD with mid-IR color space for compact group galaxies. Utilizing redshifts available from SDSS, we identified new galaxy members for 6 groups. By combining optical and mid-IR data, we obtain information on both the dust and the stellar populations in compact group galaxies. We also compare with more isolated galaxies and galaxies in the Coma cluster, which reveals that, similar to clusters, compact groups are dominated by optically red galaxies. While we find that compact group transition galaxies lie on the optical red sequence, LVL+SINGS mid-IR transition galaxies span the range of optical colors. The dearth of mid-IR transition galaxies in compact groups may be due to a lack of moderately star forming low mass galaxies; the relative lack of these galaxies could be due to their relatively small gravitational potential wells. This makes them more susceptible to this dynamic environment, thus causing them to more easily lose gas or be accreted by larger members.

In this work, I guided an undergraduate student, Natalie Butterfield, in performing the photometry. We then shared the work of the analysis as I taught her to code during the course of this project - converting the data units to physically meaningful units, culling the samples to meet our criteria, and creating the plots shown in this chapter. 
I took over the analysis when she graduated and started graduate school elsewhere, and led the interpretation of the results. Natalie contributed to the writing, but I wrote the majority of the chapter.

\subsection{Introduction}

Characterizing the star formation activity of compact group galaxies is crucial to understanding how this environment affects galaxy evolution. Johnson et al. (2007) studied the mid-infrared IRAC $(3.6-8.0 \mu \mathrm{m})$ colors of galaxies in 12 compact groups to understand their star formation properties. Their results were surprising: they found a "gap" in mid-infrared (mid-IR) color space between galaxies with colors of quiescent stellar populations and galaxies with colors indicative of strong star-forming activity. Further work by Tzanavaris et al. (2010) studied galaxies from 11 of the 12 compact groups and found a gap in their specific star formation rates (star formation rate per unit stellar mass).

Walker et al. (2012) extended the Johnson et al. (2007) study to a larger sample of 49 compact groups containing 174 galaxies. They found that the underdensity of mid-IR transition galaxies persists in a smaller region of mid-IR color space that they call the "canyon." This dearth of galaxies is not present in comparison samples of isolated galaxies, the center of Coma, or interacting pairs. However, the Coma infall region shows a similar distribution in IRAC color space to compact group galaxies. This is interpreted as a similarity between the environment in compact groups and the Coma infall region, both having high densities and reservoirs of unprocessed gas.

The mid-IR colors of galaxies only reveal their current specific star formation activity as manifest in stellar light reprocessed by dust. In order to more rigorously investigate their recent star formation history, we must also consider their optical 
colors - whether they fall in the red sequence, blue cloud, or green valley in the optical color-magnitude diagram (CMD). CMD galaxy distributions are known to be strongly dependent on galaxy environment. CMDs of field galaxies tend to have both a strong blue cloud of actively star-forming galaxies and a red sequence of "red and dead" galaxies, with an under density of galaxies falling in the green valley. This shape differs strongly from CMDs of cluster galaxies, which are dominated by the red sequence, with few galaxies in the blue cloud or green valley. Placing the compact group CMD within the context of CMDs from other environments can yield insight into galaxy evolution in these important environments.

One of the more interesting regions in a CMD is the green valley between active and quiescent galaxies (Wyder et al. 2007; Martin et al. 2007), thought to be a transition region of galaxies in which star formation has recently ceased (Thilker et al. 2010). As the young, blue, massive stars become more scarce, galaxies cross the green valley and enter the red sequence (Wyder et al. 2007). The underdensity of galaxies in the green valley is thought to be due to the short crossing time between the blue cloud and red sequence, which should be on the order of a B star's lifetime (Thilker et al. 2010). Comparison of the optical and mid-IR colors of compact group galaxies will reveal whether the mid-IR transition galaxies fall in the optical green valley. This would then indicate an intrinsic relationship between the ionizing UV photons from young OB stars and the heating of dust seen in the mid-IR. In particular, comparing the distribution of galaxies in the optical CMD and mid-IR colorspace can reveal if and how the transition of stellar populations is related to the transition of the interstellar medium.

To form a more comprehensive picture of star formation in compact groups, we have embarked on a study of the optical properties of 99 compact groups drawn from 
two catalogs, as discussed in section 4.2.1. In this paper, we present the optical CMD of this sample along with three comparison samples, and compare the optical and mid-IR colors to understand the evolution of star formation in compact groups. In particular, we wish to address the question of whether the mid-IR transition galaxies correspond to the optical green valley.

\subsection{Data}

\subsubsection{The Sample}

\section{Compact Groups}

For this study we compiled a sample of groups from the Hickson Compact Group catalog (HCG; Hickson 1982) and Redshift Survey Compact Group catalog (RSCG; Barton et al. 1996). One significant change from the criteria used in Hickson (1982) is the lack of an isolation requirement. Due to the absence of this criterion there arises the possibility of RSCGs being embedded in larger structures. The most extreme examples of this are RSCGs 67 and 68, embedded in the Coma cluster, and RSCG 21, embedded in the Perseus cluster; we have excluded these groups from our analysis. Other than the surrounding environment, RSCGs seem to be quite similar to HCGs, and their mid-IR colorspace distributions are consistent.

To be consistent with the sample from Walker et al. (2012), we included all HCGs and RSCGs (excluding RSCGs 21, 67, and 68) at $z<0.035$ (to ensure the midIR polycyclic aromatic hydrocarbon features remain in their rest-frame bands) that were available through the SDSS DR8 archive (Aihara et al. 2011). This selection process yielded 28 HCGs, 58 RSCGs, and 13 groups that are in both catalogs. Groups that appear in both the HCG and RSCG catalogs are considered only once in our 
analysis, and will be identified by their HCG designation. In addition, utilizing redshifts available from the SDSS archive, we identified new galaxy members for 6 groups. To be considered part of the group, we required that new members be located between original group members in both projected position and redshift.

\section{Comparison Samples}

In order to study the effect of galaxy environment, we compare the compact group sample with galaxies from the Coma cluster (Mahajan et al. 2010) as well as two samples of field galaxies: the first from the low-z survey of the NYU value-added galaxy catalog (VAGC; Blanton et al. 2005b,a; Adelman-McCarthy et al. 2008; Padmanabhan et al. 2008); the second is LVL+SINGS (Dale et al. 2007, 2009), this sample provides the opportunity to compare optical and mid-IR data (to be consistent with Walker et al. 2012, we only include galaxies above $\left.\log \left(L_{4.5}\left[\operatorname{erg~s}^{-1} \mathrm{~Hz}^{-1}\right]\right)=27.5\right)$. To maintain consistency with our compact group sample, we only included galaxies at $z<0.035$. Comparing the CMDs of these different samples will help us understand the effect of the compact group environment on the interstellar medium and star formation of galaxies.

\subsubsection{Completeness}

Because the samples used here do not have rigorous completeness limits, it is important to investigate what effect incompleteness in one or more of the samples might have on the results and interpretation. The HCG catalog is complete down to $m_{G}<13.0$ (Hickson 1982), while the RSCG catalog is magnitude limited to $m_{B_{0}} \leq 15.5$ (Barton et al. 1996). The faintest systems in the LVL sample approach $m_{B}=19$, but the majority of the sample is at $m_{B}<15$ (Dale et al. 2009), while the 
SINGS galaxies span $-23.5<M_{R}<-12.5$ (Dale et al. 2007). Both the VAGC and the Coma samples are constrained by the SDSS spectroscopy limit of $r<17.77$ (Blanton et al. 2005a; Mahajan et al. 2010); we note that the VAGC sample is over $95 \%$ complete over the relevant magnitude range except at very low surface brightness. For simplicity, we compare the $M_{r}$ distributions in Figure 4.1. Despite not having rigorous magnitude limits, it is clear that the LVL+SINGS and compact group samples are well matched. For Coma, there is a more noticeable difference, as the compact group sample includes more low-luminosity galaxies. The difference with VAGC is pronounced, in particular a straight comparison of galaxy populations between this catalog and compact groups at $M_{r}<-19.5$ should be done with care.

\subsubsection{Photometry}

In addition to performing our own photometry, we also conducted a consistency check against the VAGC. As it was obtained as part of a survey, the Sloan photometry was performed in a fundamentally different manner. Rather than customizing the aperture shape for each individual galaxy, the Petrosian fluxes were measured using a circular aperture of 2 Petrosian radii. However, this does not always account for all the light from a galaxy; for instance, only $80 \%$ of the flux is measured for a de Vaucouleurs profile (Blanton et al. 2001). For irregular morphologies, such as those found in compact groups, this problem is likely to be exacerbated.

Photometry was performed with SURPHOT (Reines et al. 2008), which determines apertures based on contour levels in a reference image (we used a sum of the gri images as these were the filters of interest to our study), then applies the aperture to each image of interest. We converted SDSS magnitudes to AB magnitudes, then applied K-corrections to z $=0$ using kcorrect v4_2 (Blanton \& Roweis 2007). 
Reddening corrections utilized the Galactic dust maps of Schlegel et al. (1998). Any obvious stars in the apertures were removed using SURPHOT; in most cases these had a negligible effect on the photometry. The low-z VAGC contains photometry for 21 of the galaxies in our compact group sample; a comparison reveals that the two methods are consistent $\left(\langle[\mathrm{g}-\mathrm{r}]\rangle=0.026, \sigma_{[\mathrm{g}-\mathrm{r}]}=0.025,\left\langle\mathrm{M}_{\mathrm{i}}\right\rangle=0.16, \sigma_{\mathrm{M}_{\mathrm{i}}}=0.13\right)$, as shown in Figure 4.2. Thus, for the 21 compact group galaxies in the VAGC, we could use the SDSS photometry in this work. However, to maintain consistency within our sample, we use our custom photometry for all galaxies.

\subsection{Results}

The main results of this paper are shown in Figure 4.3; the compact group sample is dominated by the optical red sequence, and the mid-IR canyon galaxies fall in a tight range on the optical red sequence, not in the green valley. These results are discussed in more detail below.

\subsubsection{Color-Magnitude Diagram}

The left panel in Figure 4.3 shows the CMD for all 348 compact group galaxies overlaid on contours representing the field sample from the VAGC. Comparison with the contours reveals that, relative to the field, compact group galaxies show a greater tendency to lie on the red sequence and form neither a distinct blue cloud nor green valley. As expected, the field galaxy sample forms a well-defined red sequence and blue cloud with the green valley wedged in between. The differences between the field and compact group samples are especially apparent in the histogram of $g-r$ color shown in Figure 4.4. This figure illustrates that the two field samples are consistent, each form both a blue cloud and red sequence. Also shown in the histograms are 


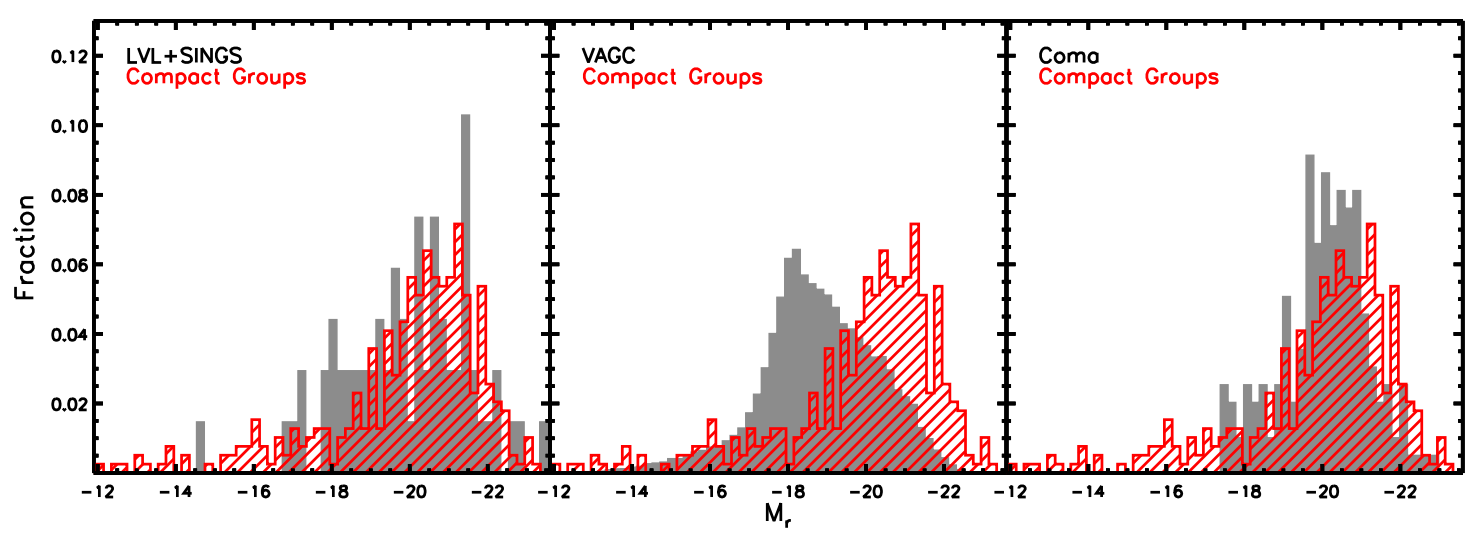

Fig. 4.1. - Comparison of the absolute magnitudes of the compact group sample and our comparison samples. The LVL+SINGS and compact group samples are well matched, both including more low-luminosity galaxies than Coma. The difference with VAGC is pronounced, and comparison at $M_{r}<-19$ should be done with care.
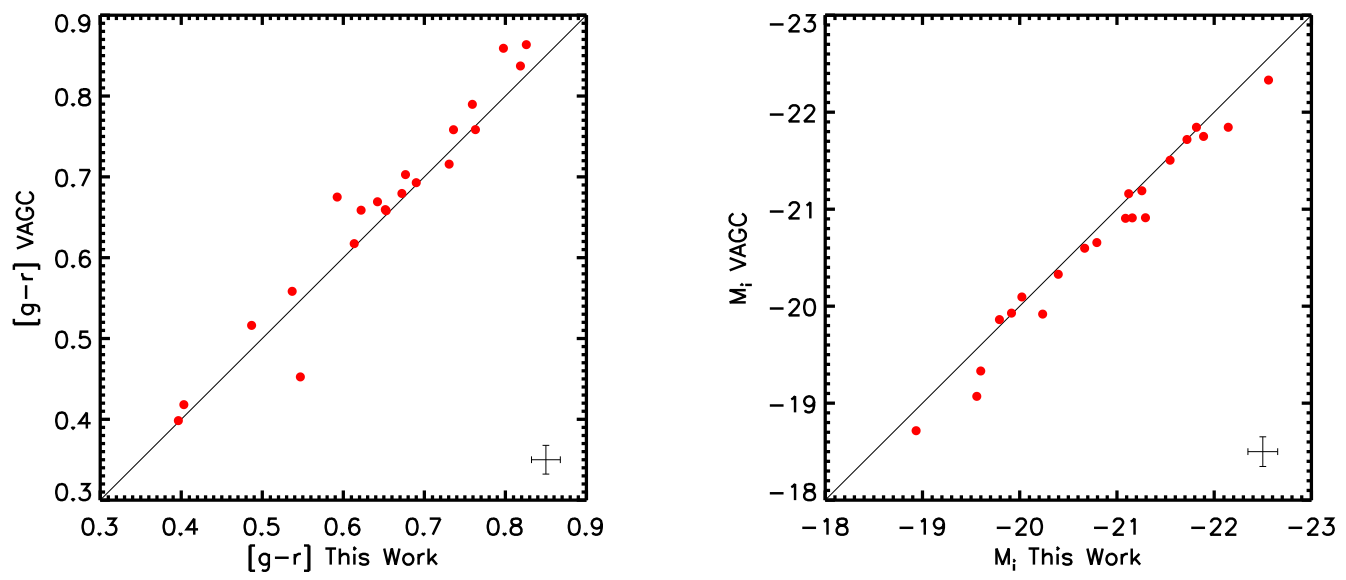

Fig. 4.2.- Comparison of our photometry with the photometry from the low-z NYU VAGC showing that the two methods are consistent. The error bars indicate the average error for our data, the line indicates equality. 

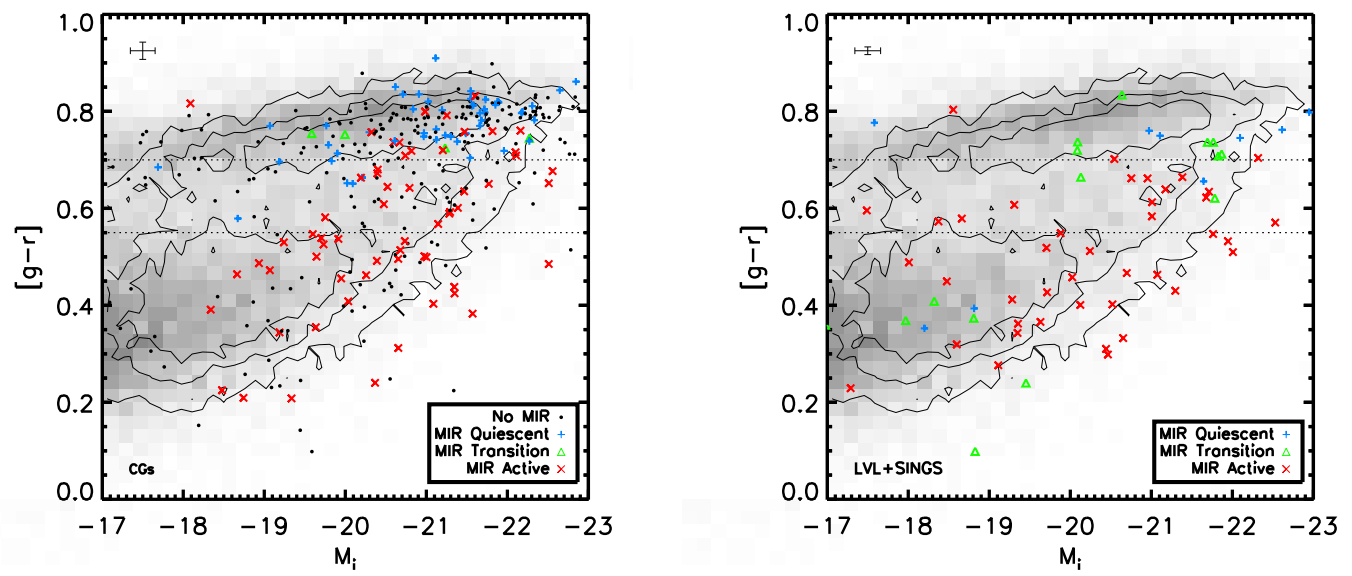

Fig. 4.3.- Color-magnitude diagram of the left: compact group sample and right: LVL+SINGS sample Dale et al. (2009) (individual points) overlaid on the VAGC Blanton et al. (2005a) (contours and grayscale). The symbols are colored by which region of mid-IR color space they fall in from Walker et al. (2012). The dotted lines indicate the bounds of the green valley. We see that the compact group sample is dominated by the optical red sequence. In compact groups, the mid-IR canyon galaxies fall in a tight range on the optical red sequence while they span the range of optical colors for LVL+SINGS. 
the colors of galaxies from the Coma cluster (Mahajan et al. 2010). It is clear that the color distribution of compact group galaxies is similar to that of Coma galaxies, though the red sequence is stronger in Coma. Comparison with CMDs from Hogg et al. (2004) reveals that the structure of the compact group CMD is similar to that of very high density regions, which is not surprising as, like clusters, compact groups are also high density.

A relatively straightforward hypothesis that mid-IR transition galaxies in compact groups occupy the green valley in the optical CMD is clearly incorrect. Instead, the mid-IR transition galaxies occupy the red sequence rather than the green valley, spanning a tight optical color range $(0.73<[g-r]<0.77)$. The mid-IR active galaxies span the full optical color range, while mid-IR quiescent galaxies tend to fall along the optical red sequence with a slight excess at optically redder colors. Note that on the right in Figure 4.3, the mid-IR transition galaxies in LVL+SINGS span the range of optical colors.

\subsubsection{Optical vs mid-IR}

By comparing the optical and mid-IR color distributions of galaxies, we can determine how the properties that give rise to mid-IR transition galaxies relate to the evolution of star formation within galaxies. If mid-IR colors track star formation, we would expect them to lead optical colors. If mid-IR colors track heating of polycyclic aromatic hydrocarbons (PAHs), we expect them to follow optical colors.

The relationship between optical and mid-IR colors for compact group and LVL+SINGS galaxies, shown in Figure 4.5, has several notable features. Galaxies that are mid-IR quiescent are optically red. By comparison, mid-IR active galaxies span the full optical color range. As seen in Figure 4.3, compact group mid-IR transition galaxies fall 
on the optical red sequence. By comparison, the LVL+SINGS sample does contain optically blue mid-IR transition galaxies, which we see from Figure 4.3 are relatively low magnitude (and likely low mass) galaxies. Their absence in compact groups is discussed in section 4.4.2. In both samples, we note a lack of galaxies that are blue in both mid-IR and optical colors; these galaxies likely recently experienced quenching, but would still have young stars present.

The total energy emitted at both optical and mid-IR wavelengths also provides insight into the global state of the galaxies. Using the SEDs of the galaxies, we determined the energy emitted at optical and infrared wavelengths by integrating $\nu L_{\nu}$ (over $4686 \AA<\lambda<7480 \AA$ and $3.6 \mu \mathrm{m}<\lambda<8.0 \mu \mathrm{m}$ ). The resulting comparison for both the compact group sample and LVL+SINGS is shown in Figure 4.6. The mid-IR transition galaxies fall on a line indicating relative optical to mid-IR emission of 8.1 ( $\sigma=1.6)$ for the compact group sample and $7.5(\sigma=3.2)$ for LVL+SINGS. Over the entire sample, the compact group galaxies show a larger scatter in this ratio than LVL+SINGS, especially for higher luminosities. The compact group sample shows a clear split in the energy plot between galaxies that are mid-IR active and mid-IR quiescent, with mid-IR quiescent galaxies tending to emit a larger fraction of energy at optical wavelengths. While not unexpected, this split is interesting because it is not nearly as apparent for LVL+SINGS where mid-IR quiescent galaxies are not clearly discernable from mid-IR transition or mid-IR active galaxies.

\subsubsection{Stars vs Dust}

The mid-IR canyon seen in compact groups also shows up clearly in a simple plot of 4.5 $\mu \mathrm{m}$ versus $8.0 \mu \mathrm{m}$ luminosity (see Figure 4.7) as a dearth of galaxies on the diagonal line of unity. These particular mid-IR bands are of interest as the $8.0 \mu \mathrm{m}$ band can 

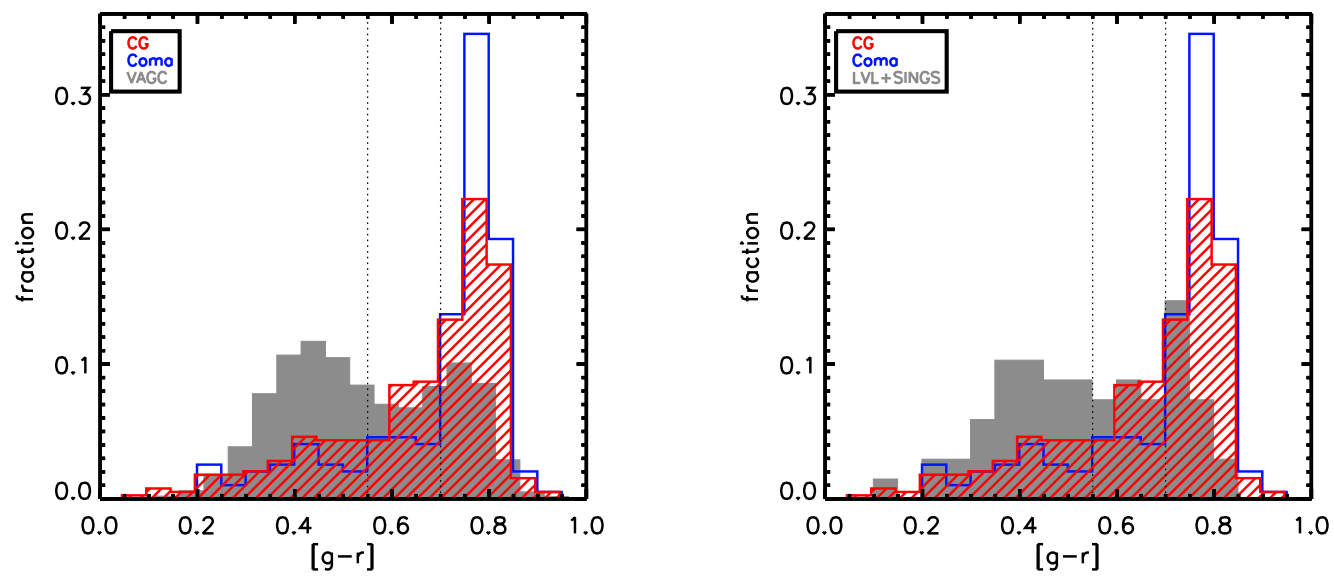

Fig. 4.4.- Histogram of g-r colors for the compact group (red stripes) and Coma samples from Mahajan et al. (2010) (blue line) overlaid on the left: VAGC Blanton et al. (2005a) and right: LVL+SINGS samples(grey solid). This clearly illustrates the dominance of the red sequence in compact groups and that our two field samples are consistent.
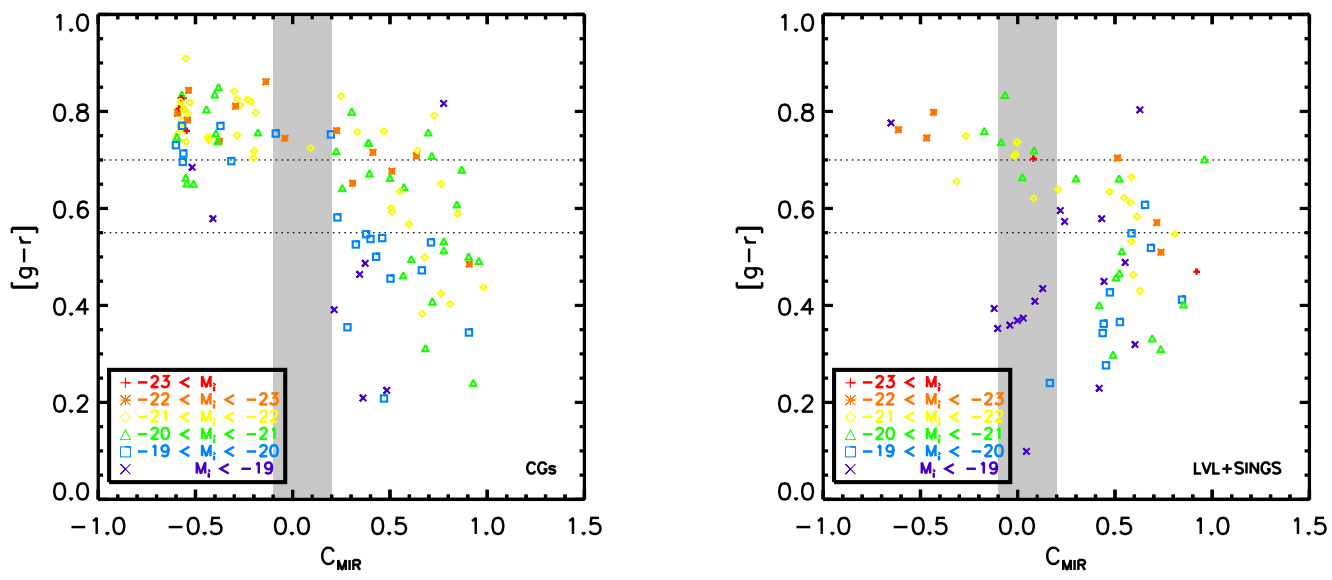

Fig. 4.5.- Comparison of optical with mid-IR colors with symbols indicating $i$-band magnitude, $\mathrm{M}_{\mathrm{i}}$ for left: compact groups and right: LVL+SINGS. $\mathrm{C}_{\text {MIR }}$ indicates midIR color along the curve from Figure 3 of Walker et al. (2012). The gray stripe indicates the canyon region from Walker et al. (2012); the green valley is between the dotted lines. The mid-IR quiescent galaxies that are optically red, while mid-IR active galaxies span the entire range of optical colors. The mid-IR transition galaxies all fall on the red sequence in compact groups, but in LVL+SINGS they span the full range of optical colors. 

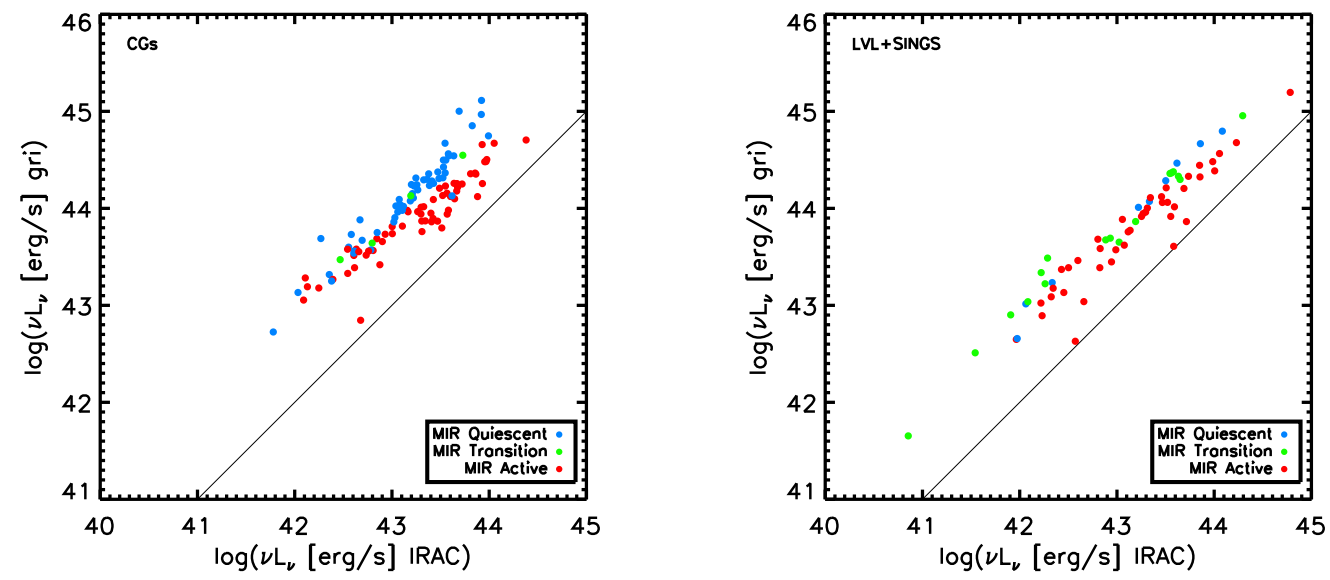

Fig. 4.6. - A comparison of the amount of light emitted in the optical vs the mid-IR for left: compact group galaxies and right: LVL+SINGS galaxies. The line indicates equality. We see a clear split between mid-IR active and mid-IR quiescent galaxies for compact groups at $\nu \mathrm{L}_{\nu}>\sim 10^{43} \mathrm{erg} / \mathrm{s}$ with an offset greater than the typical errors of $\sim 10 \%$, with mid-IR quiescent galaxies tending to emit a larger fraction of energy at optical wavelengths.

Table 4.1. Galaxies in the mid-IR Canyon

\begin{tabular}{cccccc}
\hline \hline Galaxy & $\begin{array}{c}\text { R.A. }^{\mathrm{a}} \\
(\mathrm{J} 2000)\end{array}$ & $\begin{array}{c}\text { Dec }^{\mathrm{a}} \\
(\mathrm{J} 2000)\end{array}$ & $\begin{array}{c}\mathrm{D}^{\mathrm{a}} \\
(\mathrm{Mpc})\end{array}$ & $\begin{array}{c}\text { Morphology }^{\mathrm{a}} \\
g-r \\
\text { mag }\end{array}$ \\
\hline H37e & $09^{\mathrm{h}} 13^{\mathrm{m}} 34^{\mathrm{s}} .0$ & $+30^{\circ} 02^{\prime} 23^{\prime \prime}$ & 92.6 & E0 & 0.75 \\
H57b & $11^{\mathrm{h}} 37^{\mathrm{m}} 43.6$ & $+22^{\circ} 00^{\prime} 35^{\prime \prime}$ & 130.5 & $\mathrm{SBb}$ & 0.74 \\
H57h & $11^{\mathrm{h}} 37^{\mathrm{m}} 50^{\mathrm{s}} .5$ & $+22^{\circ} 00^{\prime} 45^{\prime \prime}$ & 133.5 & SBb & 0.75 \\
H79b & $15^{\mathrm{h}} 59^{\mathrm{m}} 12^{\mathrm{s}} .5$ & $+20^{\circ} 45^{\prime} 48^{\prime \prime}$ & 69.4 & S0pec & 0.72 \\
\hline
\end{tabular}

${ }^{a}$ Galaxy RA, Dec, distance (corrected for Virgo+GA+Shapley), and morphology taken from NED. The cosmology used was $H_{0}=$ $73 \mathrm{~km} \mathrm{~s}^{-1} \mathrm{Mpc}^{-1}, \Omega_{\text {matter }}=0.27$, and $\Omega_{\text {vacuum }}=0.73$. 
be dominated by PAH emission, while the $4.5 \mu \mathrm{m}$ band is typically dominated by stars and is virtually free of PAH emission. Thus, a direct comparison of these bands can serve as an indicator of the relative contribution of PAH emission to the SED of a galaxy. Of particular note is the fact that the mid-IR transition galaxies essentially lie on the unity line, indicating these galaxies are emitting equivalent energies in the $4.5 \mu \mathrm{m}$ and $8.0 \mu \mathrm{m}$ bands. For LVL+SINGS, the mid-IR transition galaxies also fall approximately on the line of unity. However, as shown previously in Walker et al. (2012) using other diagnostics, no dearth of galaxies in LVL+SINGS is apparent in the mid-IR canyon.

This comparison may indicate that the rapid transition of compact group galaxies through the mid-IR canyon is strongly related to their PAH emission. This finding is consistent with the hypothesis that the mid-IR canyon in the compact group sample is due to global star formation being truncated on very short timescales. Alternately, the canyon being tied to PAH emission could indicate modest accretion events from the intragroup medium that provide small amounts of gas and dust for a short period of time.

\subsection{Discussion}

\subsubsection{Transition Galaxies in Optical and mid-IR}

As the green valley is considered to be a "transition region" between star forming galaxies in the blue cloud and passively evolving galaxies on the red sequence, a straightforward hypothesis would be that this region of the optical CMD corresponds to the mid-IR canyon. However, this hypothesis is clearly incorrect, as the tight range of optical colors occupied by the mid-IR transition galaxies in compact groups cor- 
responds to the optical red sequence. Thus, mid-IR transition galaxies in compact groups have optical colors that indicate a "red and dead" population, a population with significant reddening from dust, or perhaps some combination thereof. However, if dust were causing significant reddening in a star-forming galaxy, then the galaxy would be very red in the mid-IR. Visual inspection indicates a smooth optical morphology with no indication of dust lanes or irregularities.

One possible interpretation is that the star formation in mid-IR transition galaxies has recently turned off (given the red optical colors), but there is still sufficient stellar emission to generate dust and PAH emission. The optical colors of mid-IR transition galaxies in compact groups imply that the optical transition precedes the mid-IR transition. This relative sequence suggests that the dust and PAHs are not being heated by the most massive stars. This is further supported by the fact that SEDs show the dust in mid-IR transition galaxies to be at an intermediate temperature (see Figure 13 of Walker et al. 2012); if these are galaxies that no longer have massive stars to heat the dust, we would expect to see the dust cooling and mid-IR emission declining.

\subsubsection{Implications for Galaxy Evolution}

Of particular note is the fact that for the sample of compact group galaxies, the midIR canyon does not correspond to the optical green valley, which indicates that midIR transition galaxies have already transitioned to the optical red sequence. There are clear indications in the compact group CMD that galaxy evolution in compact groups is markedly different than secular evolution in the field. The increased fraction of galaxies with redder optical colors (a similarity with the Coma CMD), combined with the fact that all of the mid-IR quiescent galaxies fall in the optical red sequence, 
seems to indicate that compact group galaxies on the whole are more evolved than field galaxies. Thus, either the compact group environment is more like the cluster environment than the field environment, or galaxies in clusters experience pre-processing in a compact group-like environment (Cortese et al. 2006).

Galaxies in clusters are known to have older stellar populations on average than those in the field (e.g. Bernardi et al. 1998; Sánchez-Blázquez et al. 2006; Gobat et al. 2008). Optical studies of cluster galaxies show strong red sequences; in fact the optical CMD for the Coma cluster (Mahajan et al. 2010) is remarkably similar to our compact group CMD. Thus, galaxy processing appears to be important even in these smaller structures. This is supported by recent findings that even slight increases in local density or small groups lead to older populations (Blanton \& Berlind 2007; Blanton \& Moustakas 2009). Compact groups, though small, show densities comparable to the centers of clusters (Hickson 1982).

A comparison of the CMDs for the compact group and LVL+SINGS galaxies leads to two scenarios. The first is that the processes involved in the decline of dust emission take place on faster timescales in compact groups than in field galaxies, but this is complicated by the presence of the low-mass, optically blue mid-IR transition galaxies in the LVL+SINGS sample. Furthermore, given how well matched the compact group and LVL+SINGS samples are in $\mathrm{M}_{\mathrm{r}}$ as shown in Figure 4.1, we find it unlikely that the lack of mid-IR transition galaxies in compact groups could be due to completeness issues. The second is that the dearth of galaxies in the mid-IR canyon may be attributed to a lack of moderately star forming, low mass galaxies. This hypothesis could be explained by compact groups being inhospitable to these galaxies; they either lose their gas very easily or are accreted by larger members of the group. The lack of low mass star-forming galaxies could in part also be due to selection - there is 
a lower limit to galaxies identified as belonging to the group, though we do not see mid-IR canyon in LVL+SINGS, which has a similar population of galaxies, as shown in Figure 4.1. Lower mass, star-forming galaxies are likely to be farther away from the core, and so again could be missed in the selection, like the star-forming dwarfs found on the outskirts of HCG 59 (Konstantopoulos et al. 2012). It is not clear which effect dominates, the dearth of mid-IR transition galaxies is likely due to some combination of these factors.

The compact group environment appears to impact galaxy evolution by being inhospitable to moderate levels of star formation; the environment either enhances or terminates star formation within member galaxies. It may also be that star formation is first enhanced in member galaxies, then terminated when the star forming gas is removed due to group interactions. Therefore, how gas is processed (e.g. through star formation or stripping) affects the properties of compact group galaxies. Thus these results indicate that the location and phase of gas in compact groups plays a crucial role in determining the evolution of compact group galaxies. 

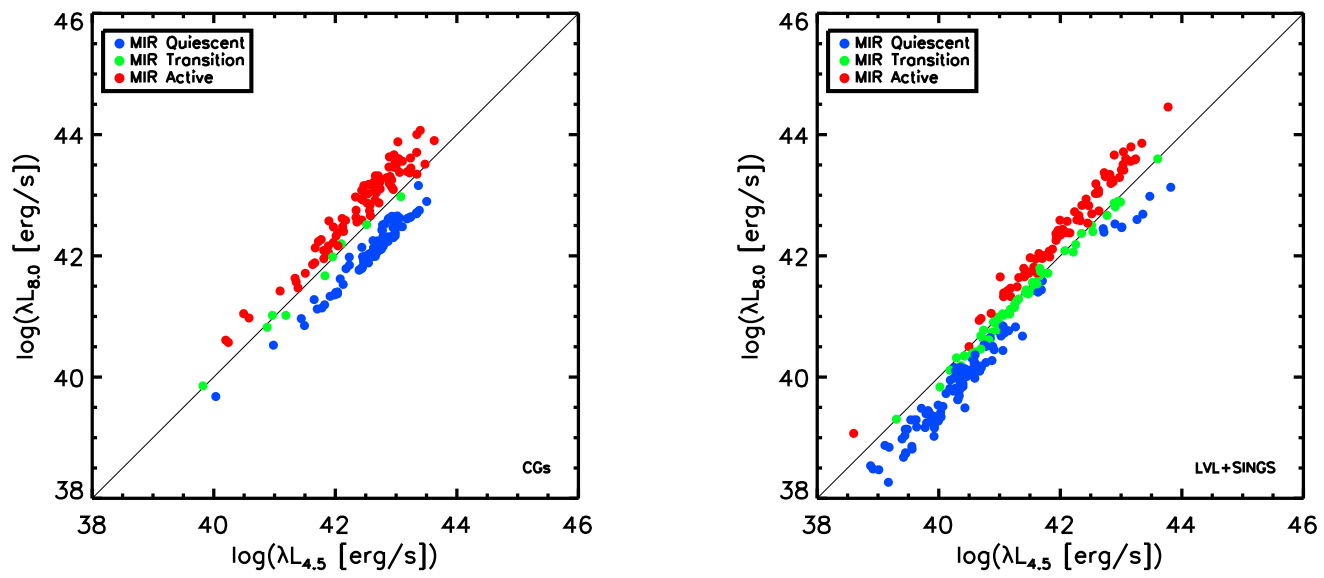

Fig. 4.7.- A comparison of the $8.0 \mu \mathrm{m}$ and $4.5 \mu \mathrm{m}$ luminosities for left: compact group galaxies and right: LVL+SINGS galaxies. Note that mid-IR transition galaxies fall on the unity line, indicating these galaxies are emitting equivalent energies in the $4.5 \mu \mathrm{m}$ (dominated by stars) and $8.0 \mu \mathrm{m}$ (potentially dominated by PAHs) bands. We also note the separation of the mid-IR-quiescent and mid-IR-active galaxies in this plot, indicating that the mid-IR bimodality is not due to behavior manifest in a single band. 
Chapter 5

Neutral Hydrogen and Galaxy

Properties in Compact Groups 


\section{Abstract}

Compact groups of galaxies provide a unique environment to study the evolution of galaxies amid continuous gravitational encounters. These nearby groups have conditions similar to those in the earlier universe when galaxies were assembled and give us the opportunity to witness hierarchical formation in progress. To understand how the compact group environment affects galaxy evolution, it is important to study the gas and dust processes in these groups. Single-dish neutral hydrogen (H I) observations of compact groups allow us to measure the H I mass of each group in our sample. We compare the H I-to-stellar mass ratio with mid-IR indicators of star formation and optical $[g-r]$ color to search for correlations between gas content and star formation activity. We find that quiescent galaxies tend to live in H I-poor groups, and galaxies with active star formation are more commonly found in H I-rich groups regardless of the location and distribution of the H I gas, though we do see "rogue" galaxies whose star formation does not correlate with group H I content. Ultimately, characterizing how the gas is processed in compact groups and the processing timescales will help us understand gas processing and the interstellar medium in environments similar to that in the earlier Universe.

For this work, I was a co-investigator or principal investigator on each proposal to obtain the data. I was the observer for the majority of the data collection, and I reduced the data using scripts I wrote in GBTIDL. I also determined the H I content using H I masses that I measured and stellar masses computed by a collaborator, Tyler Desjardins. Furthermore, I performed the analysis, creating and interpreting each set of plots. Finally, I wrote the chapter. 


\section{$5.1 \quad$ Introduction}

Previous work on compact groups has revealed that galaxies show a canyon between quiescent and active galaxies in mid-infrared (mid-IR) colorspace which is unique to the compact group environment (Johnson et al. 2007; Gallagher et al. 2008; Walker et al. 2010, 2012). This dearth is also seen in the specific star formation rates of galaxies in compact groups (Tzanavaris et al. 2010). Though likely a transition region, the mid-IR canyon does not correspond to the optical "green valley" between starforming and quiescent galaxies; in fact these mid-IR transition galaxies fall on the optical red sequence $(0.73<[g-r]<0.77)$ (Walker et al. 2013). The role of gas processing is key to this transformation. Cluver et al. (2013) found extreme $\mathrm{H}_{2}$ excitation in compact group galaxies in or near the canyon, further evidence that changing gas properties rapidly extinguish star formation. It appears as though the mid-IR canyon may result from interactions enhancing star formation, followed by rapid truncation due to a lack of fuel. Unlike simple two-body mergers, the fuel depletion might be enhanced by a variety of processes, including stripping or heating. As the environment in compact groups are similar to those of the earlier universe, this has implications for galaxy evolution at higher redshift. To determine the cause of the rapid truncation of star formation in this high density environment we must "follow the gas" in compact groups (see Thibert et al. in prep, for a study of the CO in H I-rich compact groups). The dominant mechanisms that both trigger and abrubtly quench star formation in compact groups will translate to a deeper understanding of galaxy evolution in the earlier universe.

Understanding galaxy evolution in the dense compact group environment requires not only characterizing each galaxy, but also the overall group properties and dynamics. Gas in these systems is key - it provides fuel for star formation, responds to 
shocking and dissipation, and influences the resulting galaxy. Desjardins et al. (2013) finds that X-ray emission in compact groups tends to be associated with individual galaxies, rather than the overall group. However, Desjardins et al. (submitted) do note that the hot gas in higher-mass systems does appear to be in a common envelope. Like clusters, compact groups that are more deficient in H I have a higher rate of detection in X-ray (Giovanelli \& Haynes 1985; Verdes-Montenegro et al. 2001). Single-dish studies of the atomic gas reveal the complexity of compact groups: as a class, compact groups are H I-deficient (Verdes-Montenegro et al. 2001); and additionally span a range of deficiencies. In this paper, we expand the work of Verdes-Montenegro et al. (2001) and Borthakur et al. (2010) to Redshift Survey Compact Groups (RSCGs) to

form a more comprehensive study of the neutral gas in compact groups, in order to compare with member galaxy properties. We have obtained high-quality spectra of 30 RSCGs with the 100m Green Bank Telescope (GBT). We present group H I masses, and compare these with stellar masses, galaxy morphology, and optical and mid-IR colors.

\subsection{Data}

\subsubsection{Sample}

RSCGs were selected by Barton et al. (1996) using a friends-of-friends algorithm to identify groups similar to Hickson Compact Groups (HCGs), which were selected by eye from Palomar Sky Survey plates (Hickson 1982). For this study, we have selected RSCGs at a redshift less than $z=0.035$ (to match the sample from Walker et al. 2012). Only a subset of these were actually observed, based on RA availability; these groups are listed in Table 5.1. In our analysis, we also utilize a sample of HCGs 
with H I masses from the literature (Verdes-Montenegro et al. 2001; Borthakur et al. 2010). As Barton et al. (1996) searched for HCG-like groups, we can interpret the H I data in the same way.

\subsubsection{H I Data}

$21 \mathrm{~cm}$ H I observations were obtained on the GBT for 30 RSCGs in June and July 2009 and February, March, April, and October 2010 through the GBT09B-011, GBT10A050, and GBT10C-022 programs. The observations utilized the L-band receiver with 9-level sampling and a $50 \mathrm{MHz}\left(>10000 \mathrm{~km} \mathrm{~s}^{-1}\right)$ bandwidth, yielding 16384 channels at $3.052 \mathrm{kHz}\left(0.631 \mathrm{~km} \mathrm{~s}^{-1}\right)$ resolution.

The observations were taken using position switching with two different off positions to be able to reject a bad off location; our sequence was OFF1-ON-ON-OFF2, with five minutes spent at each position. At the beginning of each run, we performed an AutoPeakFocus to minimize pointing errors. The flux calibrators 3C48 (16.2 Jy), 3C249.1 (2.26 Jy), and 3C286 (14.6 Jy) gave an average antenna gain of $1.19 \mathrm{~K} / \mathrm{Jy}$.

Reduction was performed using GBTIDL. Radio-frequency interference (RFI) flagging was performed as necessary on individual integrations. When RFI was confined to specific channels, those channels were removed in all scans for that group to prevent incorrect weighting. All polarizations and scans for each group were combined, weighted by exposure time, frequency resolution, and system temperature. The spectra for each group were then boxcar smoothed by 20 channels and a baseline was subtracted to yield the final spectrum, with $12.6 \mathrm{~km} \mathrm{~s}^{-1}$ resolution. The $\mathrm{H}$ I flux was then measured using the GBTIDL procedure gmoment over channels containing emission. 
Table 5.1. Groups in H I Survey

\begin{tabular}{|c|c|c|c|c|}
\hline RSCG & $\mathrm{N}$ & $\begin{array}{c}\theta_{G} \\
{[\operatorname{arcmin}]}\end{array}$ & $\begin{array}{c}\mathrm{D}^{\mathrm{a}} \\
{[\mathrm{Mpc}]}\end{array}$ & $\begin{array}{c}\text { Member Velocities }{ }^{\mathrm{a}} \\
{\left[\mathrm{km} \mathrm{s}^{-1}\right]}\end{array}$ \\
\hline 4 & 3 & 1.02 & $89.0 \pm 6.2$ & $6664,6787,6798$ \\
\hline 5 & 5 & 3.40 & $68.4 \pm 4.8$ & $4233,4975,5098,5229,5556$ \\
\hline 6 & 3 & 1.63 & $69.1 \pm 4.8$ & $5188,5344,5373$ \\
\hline 7 & 3 & 2.94 & $60.4 \pm 4.2$ & $4114,4399,5010$ \\
\hline 8 & 5 & 3.66 & $68.3 \pm 4.8$ & $4226,4934,5019,5526,5922$ \\
\hline 10 & 3 & 1.90 & $67.8 \pm 4.7$ & $4854,5470,5785$ \\
\hline 11 & 5 & 2.14 & $71.8 \pm 5.0$ & $4070,5296,5338,5468,5533$ \\
\hline 14 & 3 & 3.83 & $40.0 \pm 2.8$ & $2800,2835,2959$ \\
\hline 15 & 4 & 2.09 & $64.3 \pm 4.5$ & $4514,4728,4855,5580$ \\
\hline 16 & 3 & 1.63 & $72.4 \pm 5.1$ & $5033,5621,5621$ \\
\hline 17 & 3 & 1.23 & $75.5 \pm 5.3$ & $5561,5969,6333$ \\
\hline 21 & 3 & 2.47 & $68.7 \pm 4.8$ & $4500,5066,5387$ \\
\hline 22 & 3 & 4.18 & $19.6 \pm 1.4$ & $1210,1524,1884$ \\
\hline 30 & 3 & 4.04 & $33.4 \pm 2.3$ & $1979,2049,2105$ \\
\hline 31 & 3 & 3.05 & $29.5 \pm 2.1$ & $1670,1673,1726$ \\
\hline 32 & 3 & 0.72 & $96.3 \pm 7.6$ & $5180,6109,6948$ \\
\hline $34^{\dagger}$ & 3 & 5.54 & $25.7 \pm 1.8$ & $1328,1566,1618$ \\
\hline $38^{\dagger}$ & 3 & 7.46 & $27.2 \pm 1.9$ & $645,1494,1586$ \\
\hline 42 & 3 & 1.27 & $93.5 \pm 6.5$ & $6190,6342,6637$ \\
\hline 44 & 6 & 3.20 & $93.6 \pm 6.6$ & $5692,6130,6316,6356,6725$ \\
\hline 45 & 3 & 0.62 & $81.4 \pm 5.7$ & $4444,5543,6406$ \\
\hline $53^{\dagger}$ & 3 & 7.34 & $14.0 \pm 1.0$ & $985,1135,1149$ \\
\hline $54^{\dagger}$ & 3 & 7.84 & $14.1 \pm 1.0$ & $697,729,922$ \\
\hline $59^{\dagger}$ & 3 & 6.94 & $14.0 \pm 1.0$ & $909,1210,1588$ \\
\hline $61^{\dagger}$ & 5 & 7.81 & $13.9 \pm 1.0$ & $757,1307,1349,1555,1970$ \\
\hline 64 & 3 & 1.50 & $73.7 \pm 5.2$ & $4716,4817,4955$ \\
\hline 67 & 3 & 0.64 & $106.4 \pm 7.5$ & $6729,7176,7193$ \\
\hline 68 & 3 & 1.76 & $98.3 \pm 6.9$ & $6392,6495,6903$ \\
\hline $78^{\dagger}$ & 4 & 4.59 & $56.9 \pm 4.0$ & $3682,3804,3865,4211$ \\
\hline 86 & 4 & 1.26 & $117.6 \pm 8.2$ & $8104,8412,9152,9267$ \\
\hline
\end{tabular}

Note. - ${ }^{\mathrm{a}}$ Group distance (corrected for Virgo+GA+Shapley) and member velocities from NED. The cosmology used is $H_{0}=$ $73 \mathrm{~km} \mathrm{~s}^{-1} \mathrm{Mpc}^{-1}, \Omega_{m a t}=0.27$, and $\Omega_{v a c}=0.73$. 'Troup extends beyond GBT FWHM. 


\subsection{Results}

We detected H I in 15 groups, shown in Figure 5.1, a detection rate of 50\%. The detection rate is artifically low because many of the baselines were not smooth polynomials and thus could not be properly subtracted; this is not indicative of the detection rate of compact groups as a whole - if we only consider the 20 with good baselines, the detection rate rises to $75 \%$. Seven of the groups with bad baselines have strong radio continuum emision in NVSS (Condon et al. 1998) images: RSCGs 5, 10, 11, 17, 61, 67, and 86. The five undetected groups with good baselines (RSCGs 8, 22, 45, 59, and 68) all have a significant fraction of early-type galaxies. As in Verdes-Montenegro et al. (2001) and Borthakur et al. (2010), fluxes were converted to H I masses using

$$
M_{H I}\left[M_{\odot}\right]=2.36 \times 10^{5}\left(\frac{D}{M p c}\right)^{2} \int\left(\frac{S_{\nu}}{J y}\right)\left(\frac{d v}{k m s^{-1}}\right) .
$$

Masses and upper limits are listed in Table 5.2. For completeness, we include HCGs in our analysis, using H I masses from Verdes-Montenegro et al. (2001) and Borthakur et al. (2010). 
Table 5.2. Masses and Upper Limits for RSCGs

\begin{tabular}{|c|c|c|c|c|c|c|}
\hline RSCG & $\begin{array}{c}M_{H I} \\
\times 10^{9} M_{\odot} \\
\end{array}$ & $\begin{array}{c}\sigma_{M_{H I}} \\
\times 10^{9} M_{\odot} \\
\end{array}$ & $\begin{array}{c}M_{*} \\
\times 10^{9} M_{\odot} \\
\end{array}$ & $\begin{array}{c}\sigma_{M_{*}} \\
\times 10^{9} M_{\odot}\end{array}$ & $\log \left(\frac{M_{H I}}{M_{*}}\right)$ & H I Type \\
\hline 4 & 3.3 & 0.5 & 473.7 & 6.6 & -2.16 & H I-poor \\
\hline $5^{\dagger}$ & $<5.9$ & & $\ldots$ & $\ldots$ & $\ldots$ & $\ldots$ \\
\hline 6 & 14.7 & 2.0 & 118.5 & 1.4 & -0.91 & H I-rich \\
\hline $7^{\dagger}$ & $<1.7$ & & $\ldots$ & $\ldots$ & $\ldots$ & $\ldots$ \\
\hline 8 & $<1.1$ & & $\ldots$ & $\ldots$ & $\ldots$ & $\ldots$ \\
\hline $10^{\dagger}$ & $<0.93$ & & $\ldots$ & $\ldots$ & $\ldots$ & $\ldots$ \\
\hline $11^{\dagger}$ & $<3.5$ & & $\ldots$ & $\ldots$ & $\ldots$ & $\ldots$ \\
\hline 14 & 10.1 & 1.4 & $\ldots$ & $\ldots$ & $\ldots$ & $\ldots$ \\
\hline $15^{\dagger}$ & $<0.91$ & & 199.0 & 1.6 & $<-2.34$ & $\ldots$ \\
\hline 16 & 1.9 & 0.3 & $\ldots$ & $\ldots$ & $\ldots$ & $\ldots$ \\
\hline $17^{\dagger}$ & $<5.4$ & & 288.4 & 2.3 & $<-1.73$ & $\ldots$ \\
\hline $21^{\dagger}$ & $<18.5$ & & 244.7 & 2.4 & $<-1.12$ & $\ldots$ \\
\hline 22 & $<0.19$ & & $\ldots$ & $\ldots$ & $\ldots$ & $\ldots$ \\
\hline 30 & 0.3 & 0.04 & $\ldots$ & $\ldots$ & $\ldots$ & $\ldots$ \\
\hline 31 & 1.8 & 0.3 & 73.0 & 0.07 & -1.60 & intermediate \\
\hline 32 & 9.1 & 1.4 & 505.1 & 1.6 & -1.75 & intermediate \\
\hline 34 & 1.8 & 0.2 & 77.8 & 0.1 & -1.64 & intermediate \\
\hline 38 & 4.0 & 0.6 & 41.1 & 0.05 & -1.01 & H I-rich \\
\hline 42 & 18.6 & 2.6 & 148.3 & 1.7 & -0.90 & H I-rich \\
\hline 44 & 3.4 & 0.5 & 514.9 & 2.1 & -2.18 & H I-poor \\
\hline 45 & $<0.65$ & & $\ldots$ & $\ldots$ & $\ldots$ & $\ldots$ \\
\hline 53 & 0.5 & 0.07 & $\ldots$ & $\ldots$ & $\ldots$ & $\ldots$ \\
\hline 54 & 0.05 & 0.006 & $\ldots$ & $\ldots$ & $\ldots$ & $\ldots$ \\
\hline 59 & $<0.035$ & & $\ldots$ & $\ldots$ & $\ldots$ & $\ldots$ \\
\hline $61^{\dagger}$ & $<5.14$ & & $\ldots$ & $\ldots$ & $\ldots$ & $\ldots$ \\
\hline 64 & 16.7 & 2.4 & 144.6 & 0.5 & -0.94 & H I-rich \\
\hline $67^{\dagger}$ & $<1.9$ & & 480.9 & 2.3 & $<-2.40$ & $\ldots$ \\
\hline 68 & $<1.0$ & & 461.2 & 3.7 & $<-2.66$ & $\ldots$ \\
\hline 78 & 13.2 & 1.9 & $\ldots$ & $\ldots$ & $\ldots$ & $\ldots$ \\
\hline $86^{\dagger}$ & $<22.4$ & & 472.3 & 2.1 & $<-1.32$ & $\ldots$ \\
\hline
\end{tabular}

Note. - H I masses and upper limits. $M_{*}$ from Desjardins et al. (submitted). ${ }^{\dagger}$ Group with unusable baseline. 

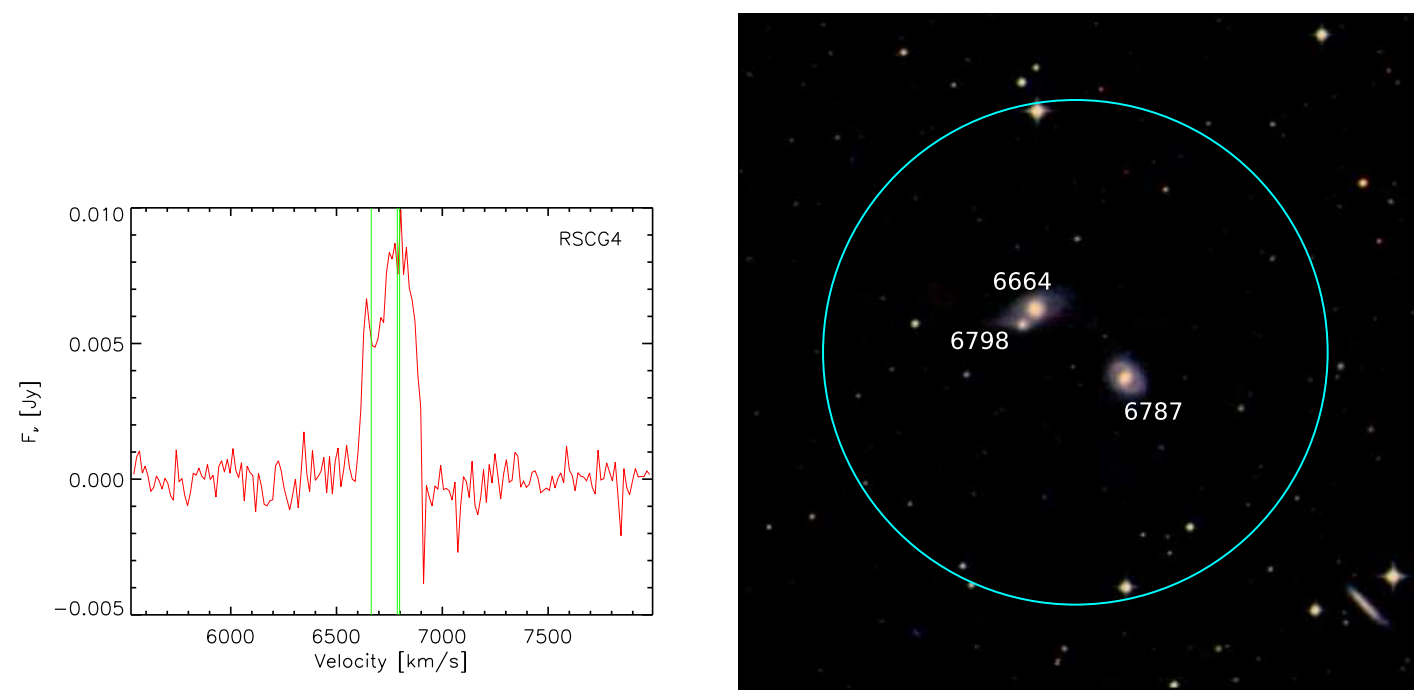

Fig. 5.1.- RSCG 4. Left: The H I spectrum. Vertical green lines indicate the velocities of member galaxies. Right: Optical image from SDSS with the GBT beam shown in cyan and galaxy velocities labelled in white. (With the exception of RSCG 4 and RSCG 16, which are outside the SDSS footprint; these images are from DSS2.)
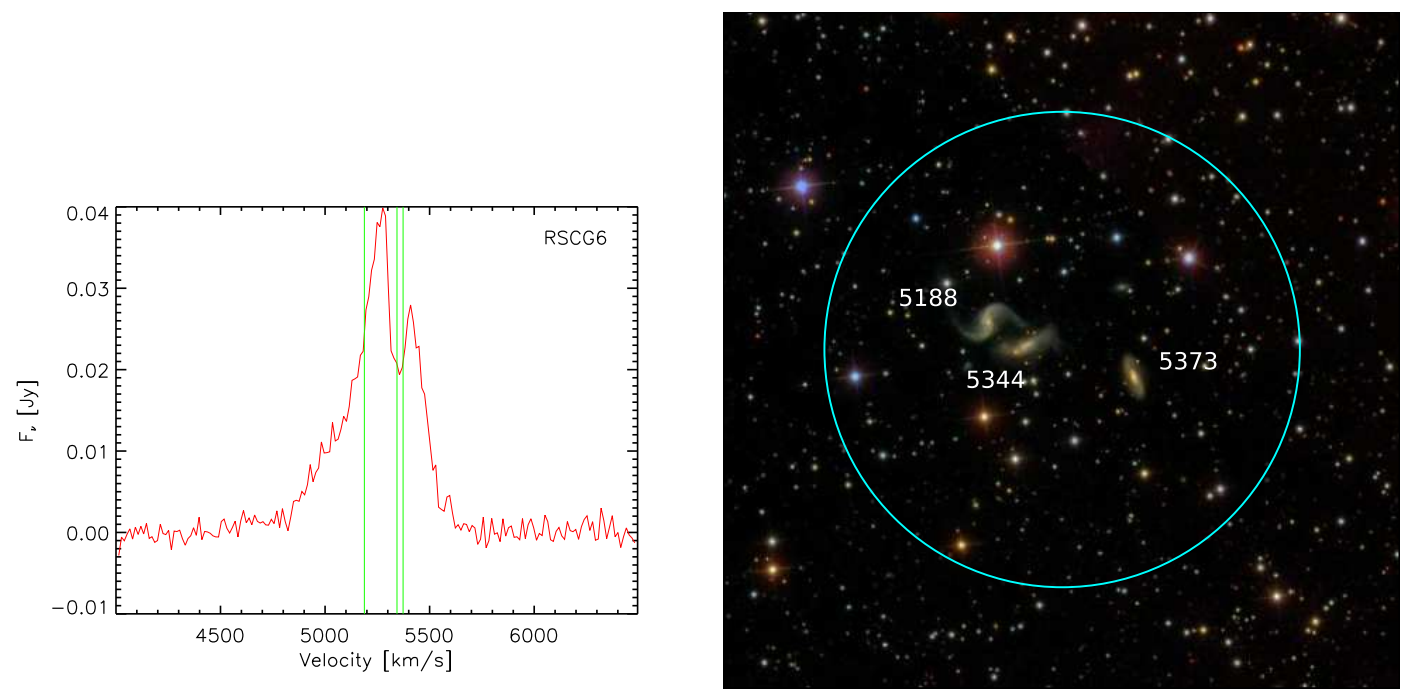

Fig. 5.1.- continued. RSCG 6 . 

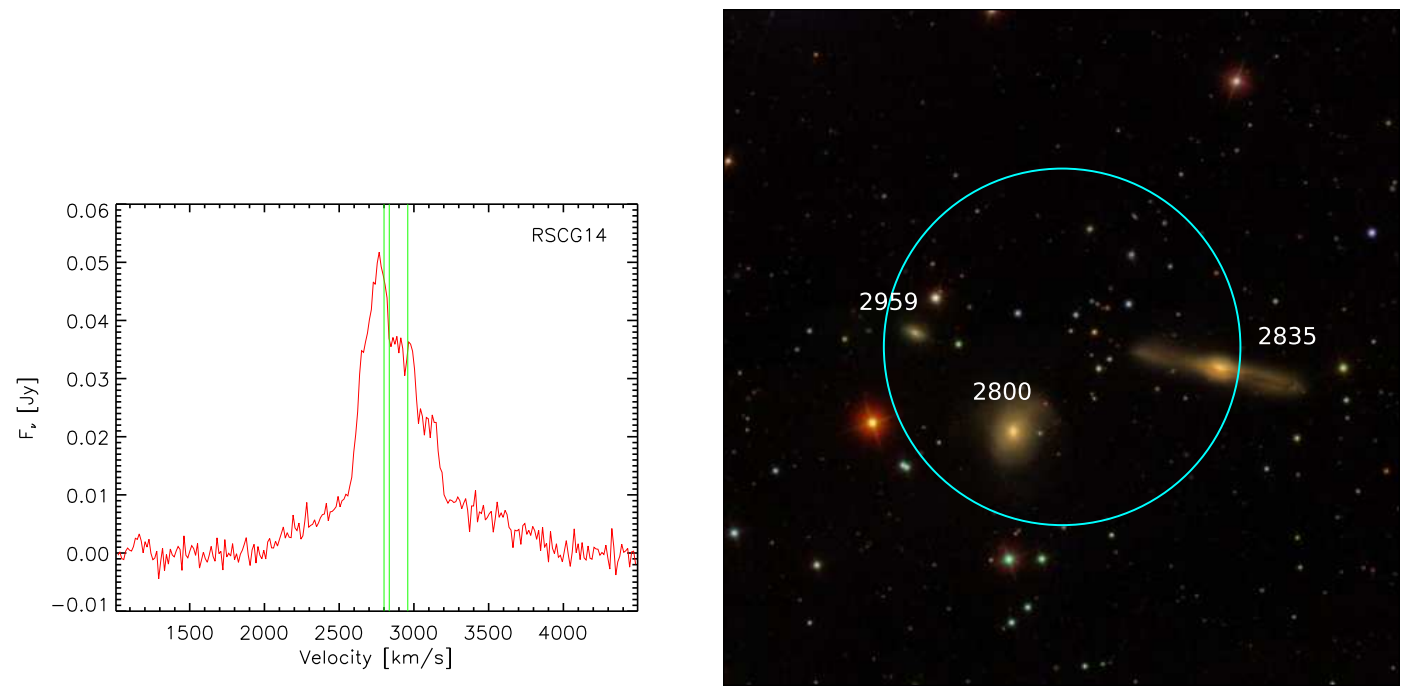

Fig. 5.1.- continued. RSCG 14.
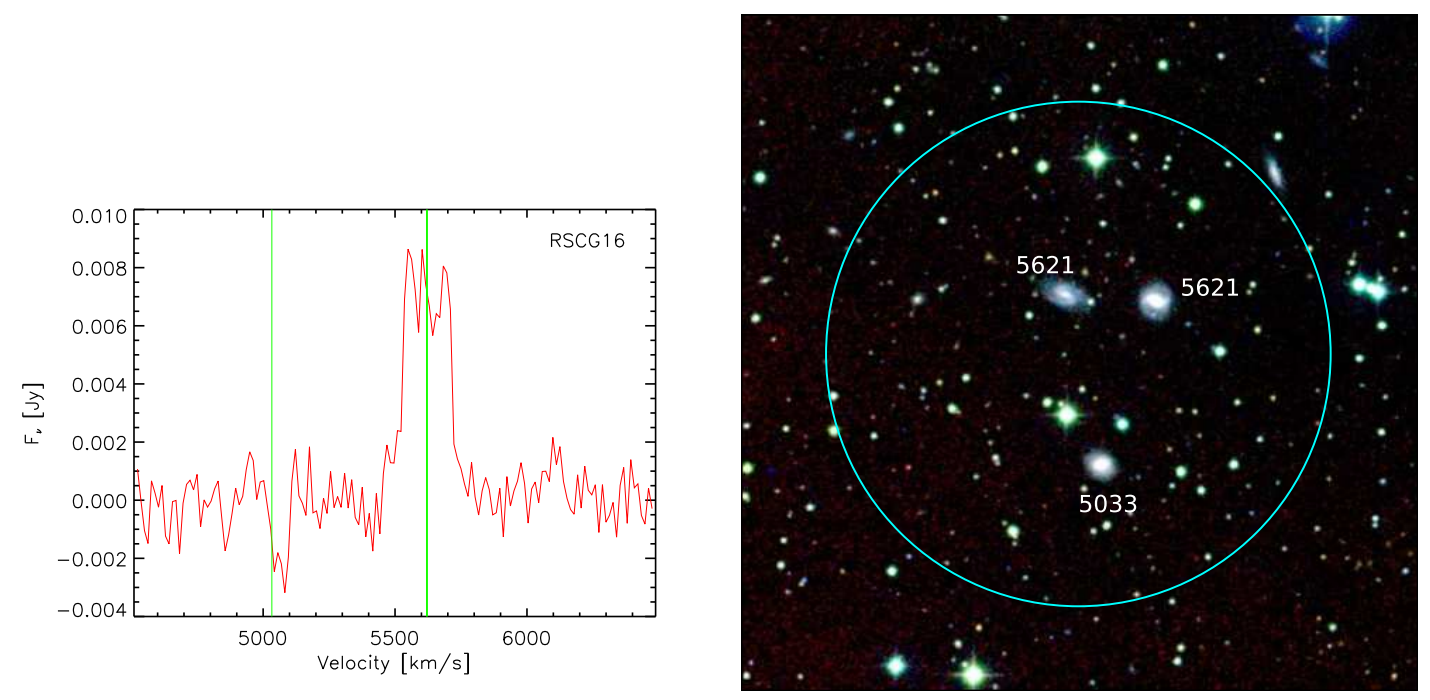

Fig. 5.1.- continued. RSCG 16. 

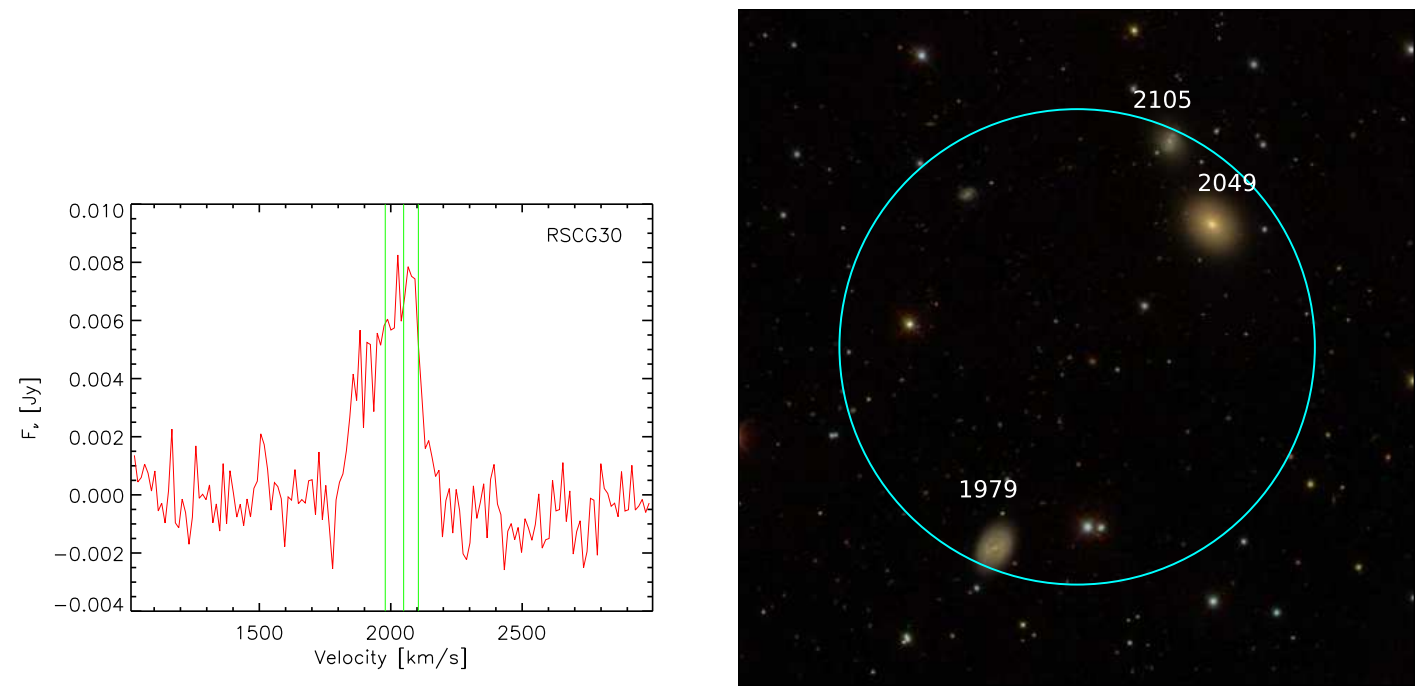

Fig. 5.1.- continued. RSCG 30.
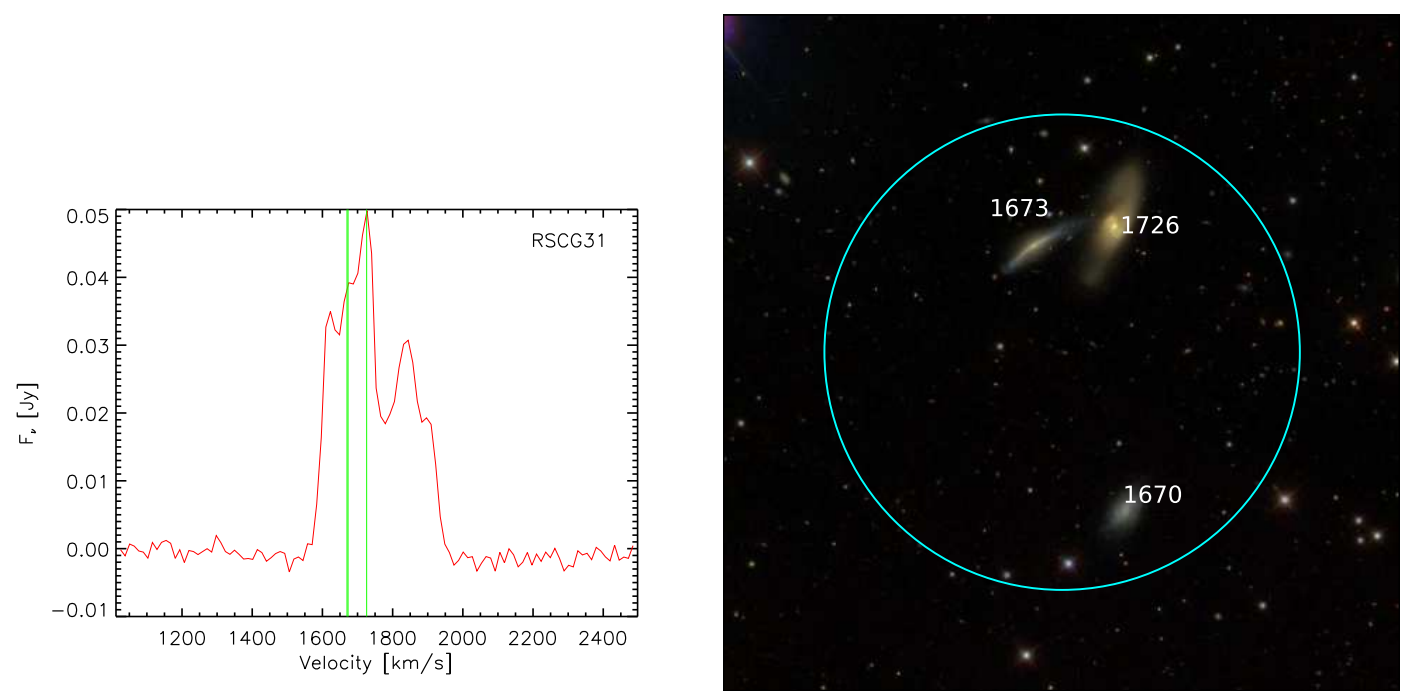

Fig. 5.1.- continued. RSCG 31. 

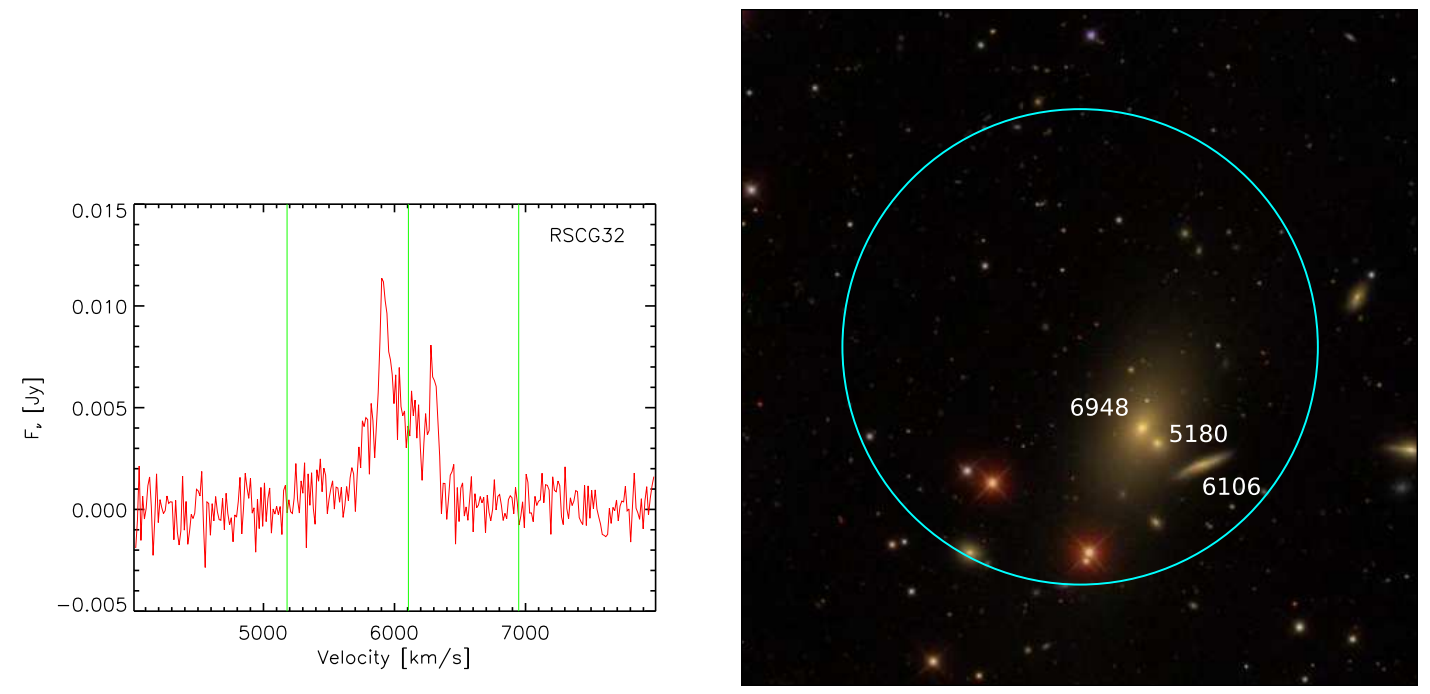

Fig. 5.1.- continued. RSCG 32.
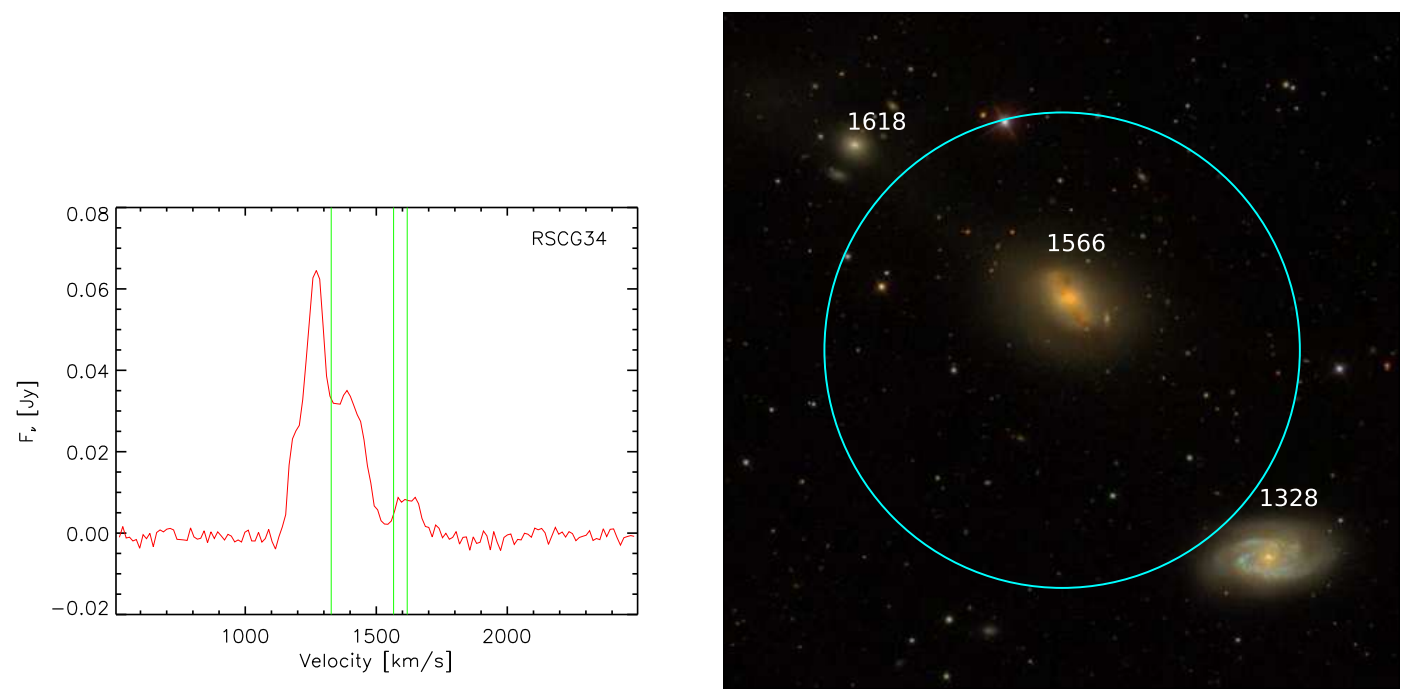

Fig. 5.1.- continued. RSCG 34. 

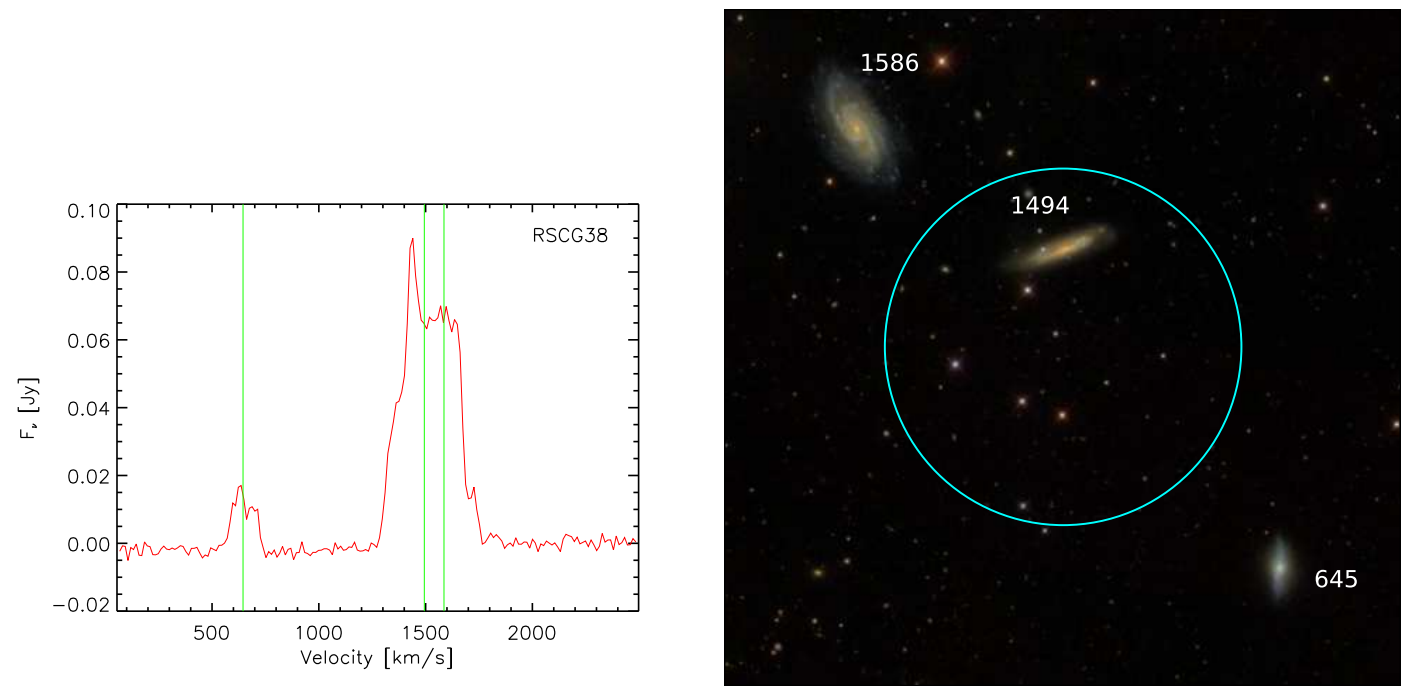

Fig. 5.1.- continued. RSCG 38.
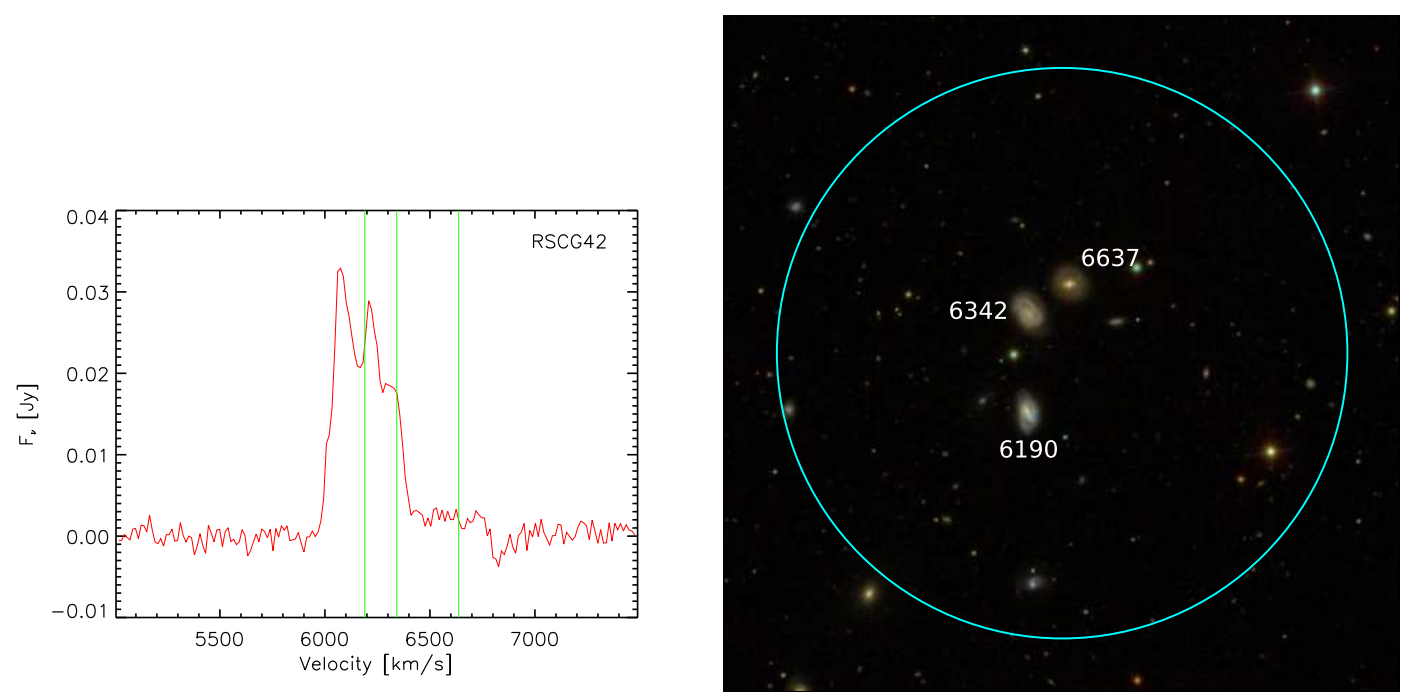

Fig. 5.1.- continued. RSCG 42. 

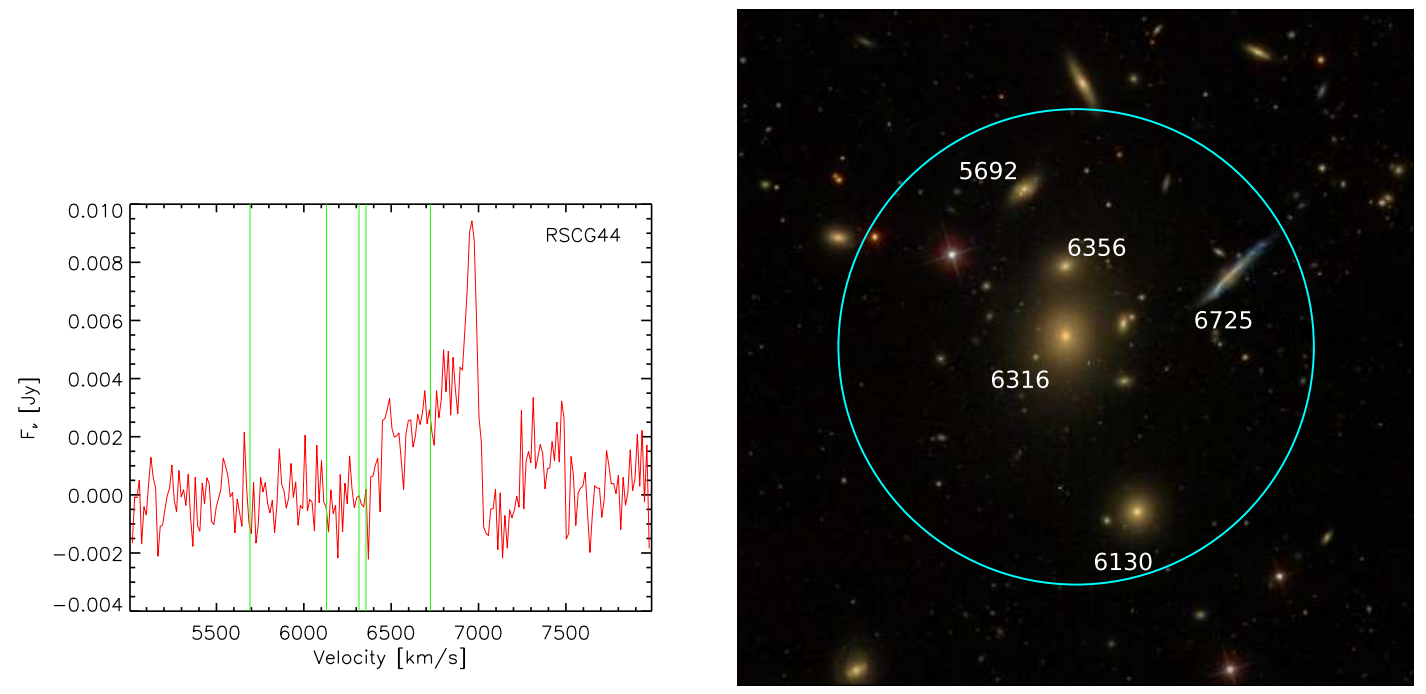

Fig. 5.1.- continued. RSCG 44.
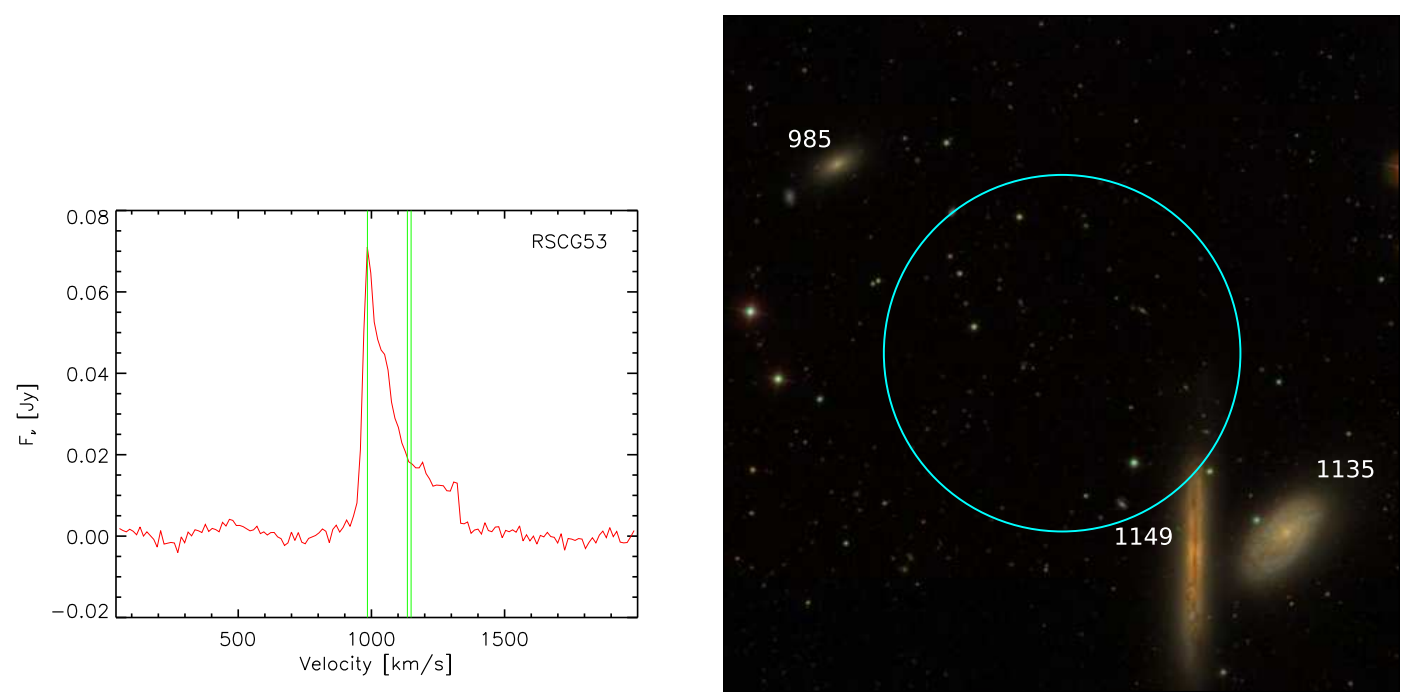

Fig. 5.1.- continued. RSCG 53. 

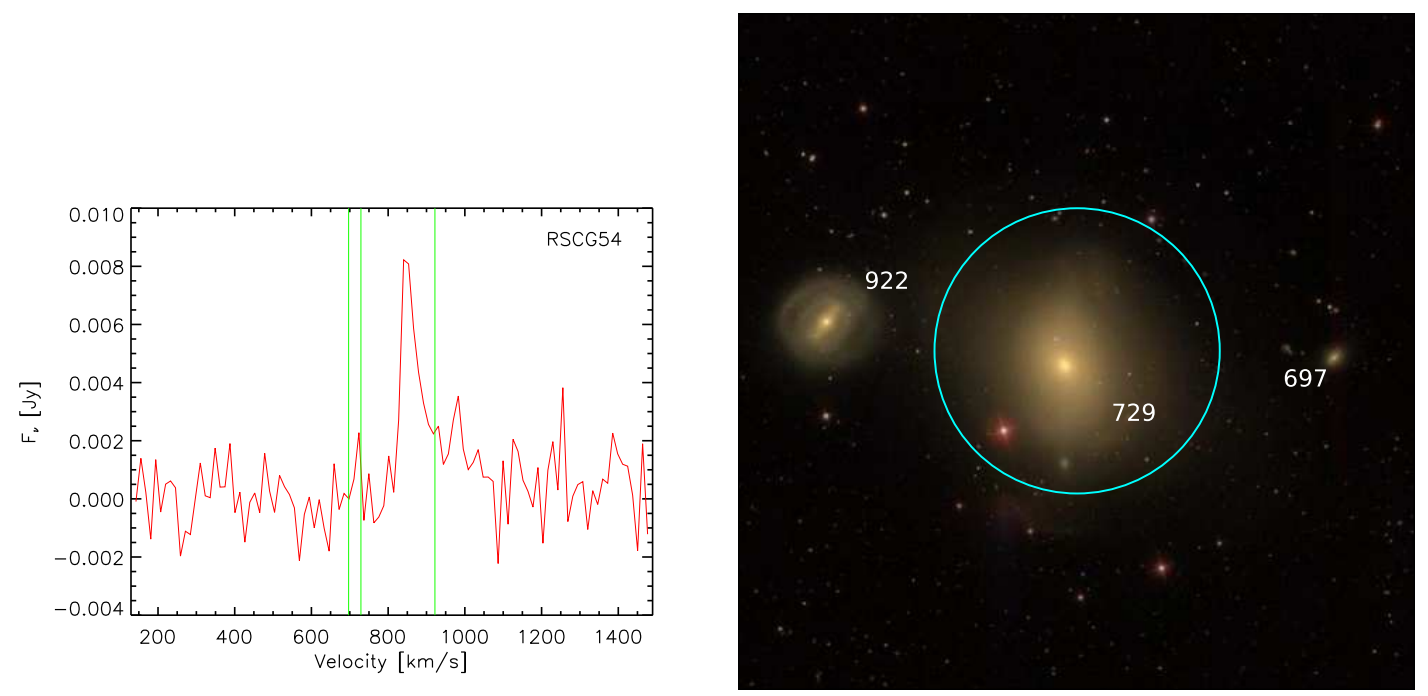

Fig. 5.1.- continued. RSCG 54.
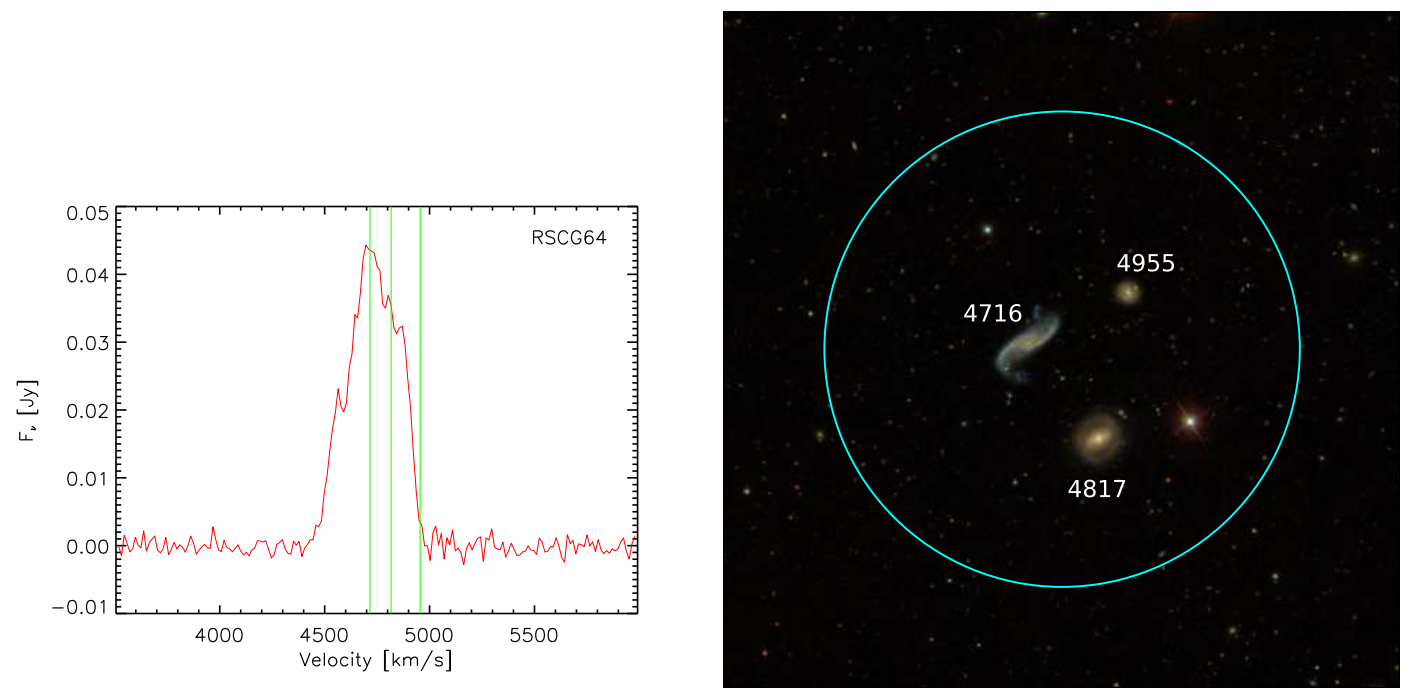

Fig. 5.1.- continued. RSCG 64 . 

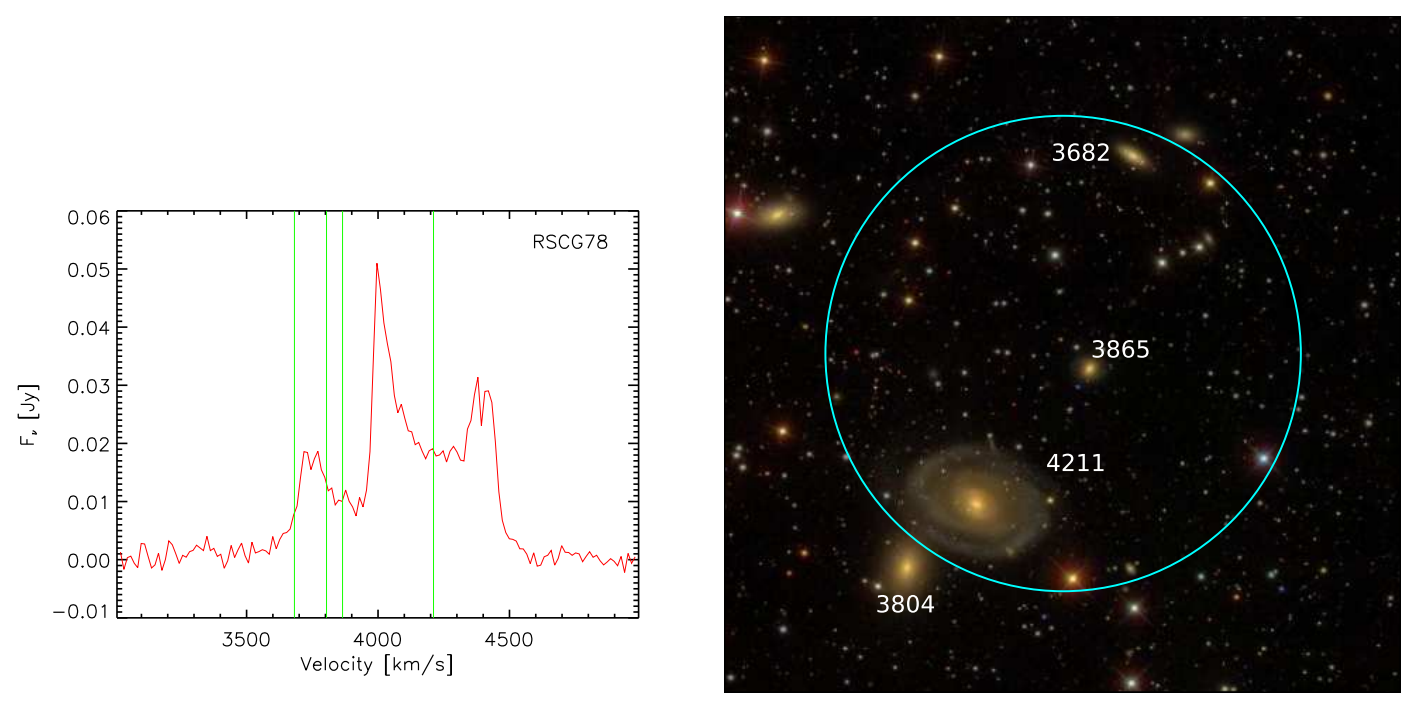

Fig. 5.1.- continued. RSCG 78. 


\subsubsection{H I Content: Comparison with Stellar Masses}

As many of these groups have only 3 known members, using the dynamical mass to determine deficiencies has little physical meaning (McConnachie et al. 2008). Another method commonly used is to determine the predicted H I mass based on a galaxy's luminosity, and sum the masses of the members to determine a predicted H I mass for the group. However, there are a number of prescriptions giving different results for how to estimate a predicted H I mass from a luminosity (e.g., Haynes \& Giovanelli 1984; Toribio et al. 2011), so this method is not ideal either. Thus, we compute H I content using group stellar masses, which is simply the sum of the stellar masses of the giant members.

Stellar masses were computed one of two ways, as in Desjardins et al. (submitted). When possible, they were determined through SED fitting of Two Micron All Sky Survey (2MASS) $J H K_{s}$ and Spitzer IRAC 3.6-8.0 $\mu m$ data using the GRASIL model galaxy spectral library (Silva et al. 1998; Silva 1999; Granato et al. 2000; Bressan et al. 2002; Silva et al. 2003; Panuzzo et al. 2003; Vega et al. 2005; Silva 2009). When these data were not available, stellar masses were computed using $K_{s}$-band luminosities and a mass-to-light ratio of 0.95 (Bell et al. (2003); for more details see Desjardins et al. submitted). The H I content is computed using group stellar and H I masses as $\log \left(\frac{M_{H I}}{M_{*}}\right)$.

A comparison of the stellar vs H I mass for both HCGs and RSCGs is shown in Figure 5.2. The H I mass is always less than the stellar mass of the group, but the difference is not a constant. The H I content for the RSCGs and the HCGs is similar as shown in Figure 5.3; the mean H I content for the RSCGs is $\log \left(\frac{M_{H I}}{M_{*}}\right)=-1.45 \pm 0.53$

and the mean H I content for the HCGs is $\log \left(\frac{M_{H I}}{M_{*}}\right)=-1.52 \pm 0.66$; the two samples have a $37 \%$ probability of being drawn from the same parent distribution. This 
provides circumstantial evidence that the different selection criteria for these samples is not affecting the global gas processing in a significant way.

\subsection{Discussion}

\subsubsection{Morphologies}

Figure 5.4 shows the H I properties as a function of the spiral fraction of the group. This shows that there is no correspondence between $M_{H_{I}}$ and spiral fraction, but the H I content of the group does appear to be higher for groups with a larger percentage of spirals. This is unsurprising, as spiral galaxies tend to be more H I-rich than early-type (elliptical or lenticular) galaxies.

\subsubsection{Mid-IR and Optical Colors}

To understand the impact of the group environment, it is important to investigate how galaxy properties are affected by group H I content. Figure 5.5 shows the mid-IR colors of galaxies in compact groups as a function of group H I content. As in Johnson et al. (2007), we see a correlation between group H I content and galaxy mid-IR color. This is not surprising, as groups with more H I have more raw fuel available for star formation. Figure 5.6 shows galaxies in the optical color-magnitude diagram (CMD), binned by the H I content of their group. Like with the mid-IR colors, there is a correlation with optical colors; the optical CMD for galaxies in H I-poor groups is similar to that for high-density environments (i.e., clusters) with a large red sequence, while galaxies in H I-rich groups occupy the optical CMD more similarly to galaxies in low-density regions (i.e., the field) with galaxies both in the blue cloud of active star formation and on the red sequence of either quiescence or dust extinction (Hogg 


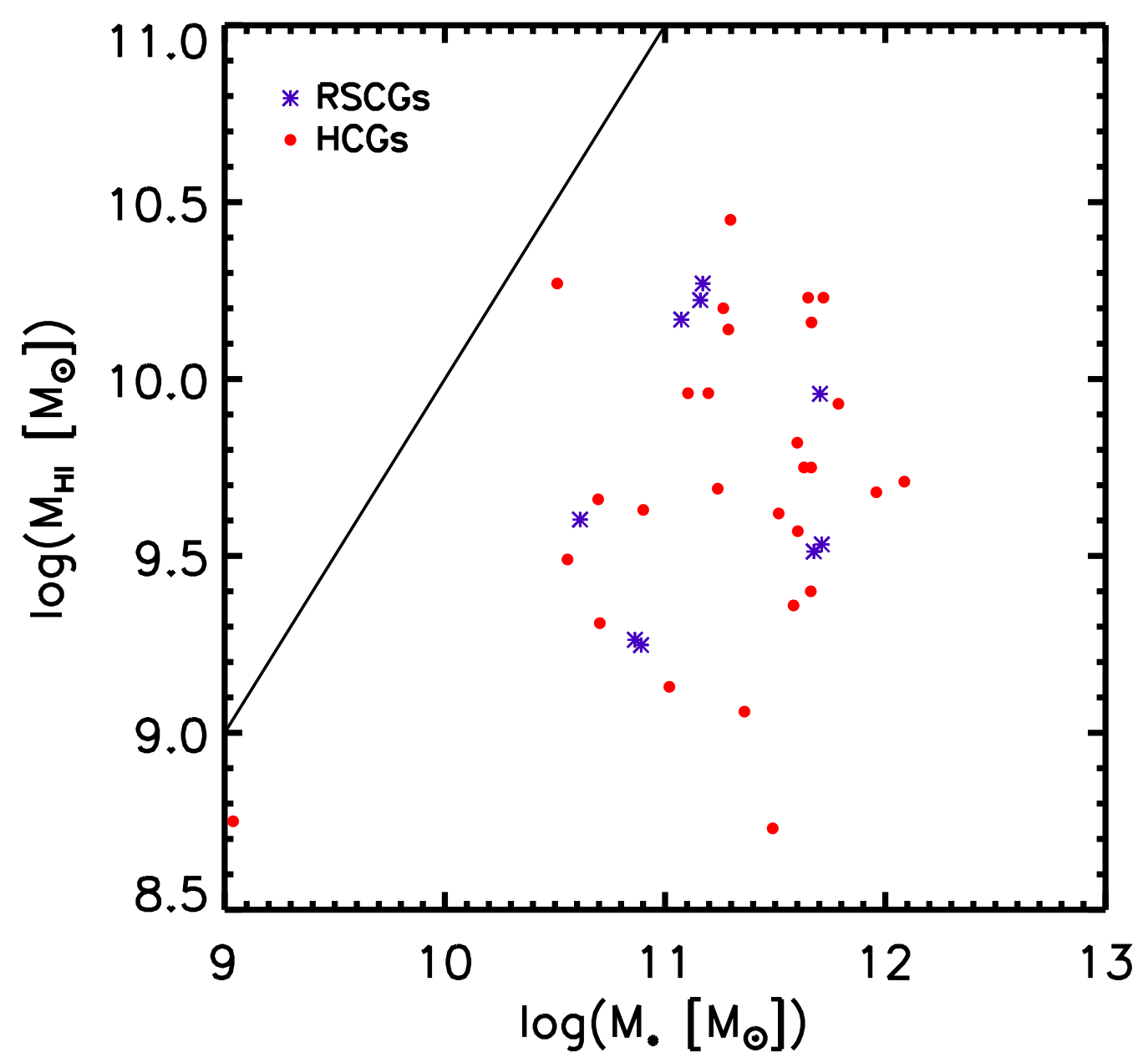

Fig. 5.2.- Comparison of $M_{H I}$ and $M_{*}$; red circles are HCGs, purple asterisks are RSCGs. These are only groups for which both H I and stellar masses were available. The solid line indicates a one-to-one correspondance. The compact groups all fall below this line, indicating that they contain more stellar mass than $\mathrm{H}$ I, but there does not seem to be a correlation between $M_{H_{I}}$ and $M_{*}$. 

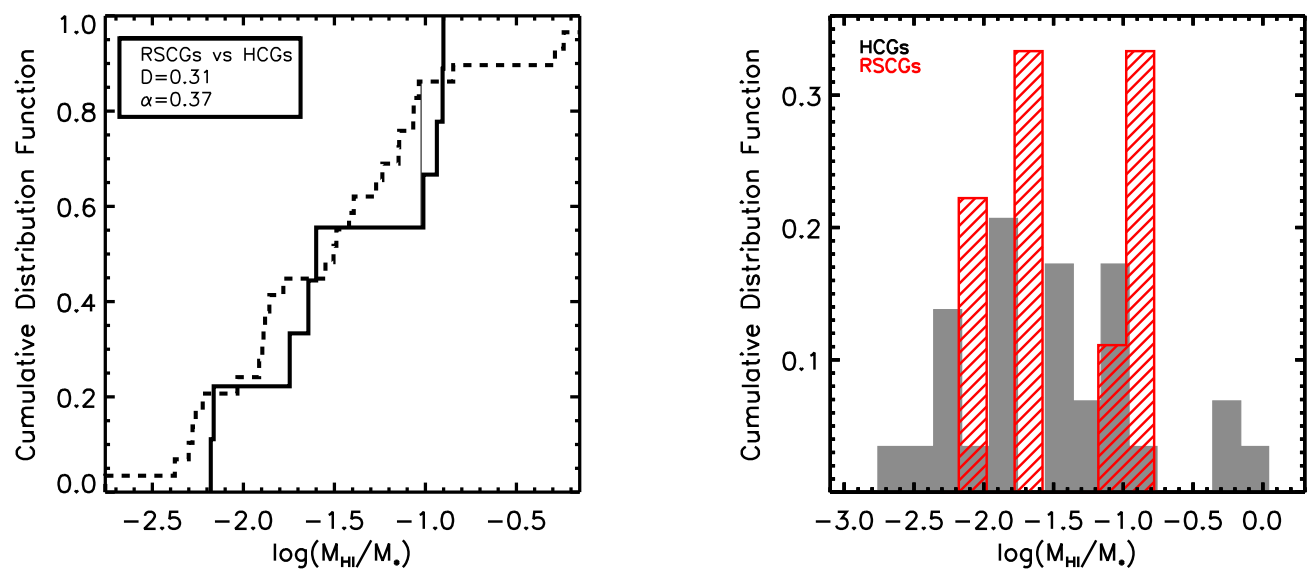

Fig. 5.3.- Left: Kolmogorov-Smirnov test comparing $\log \left(\frac{M_{H I}}{M_{*}}\right)$ for the two samples; the solid line indicates the RSCGs while the dotted line represents the HCGs. The samples are quite similar, with a $37 \%$ probability of being drawn from the same parent distribution. Right: Histogram of $\log \left(\frac{M_{H I}}{M_{*}}\right)$, showing the HCGs and RSCGs separately.
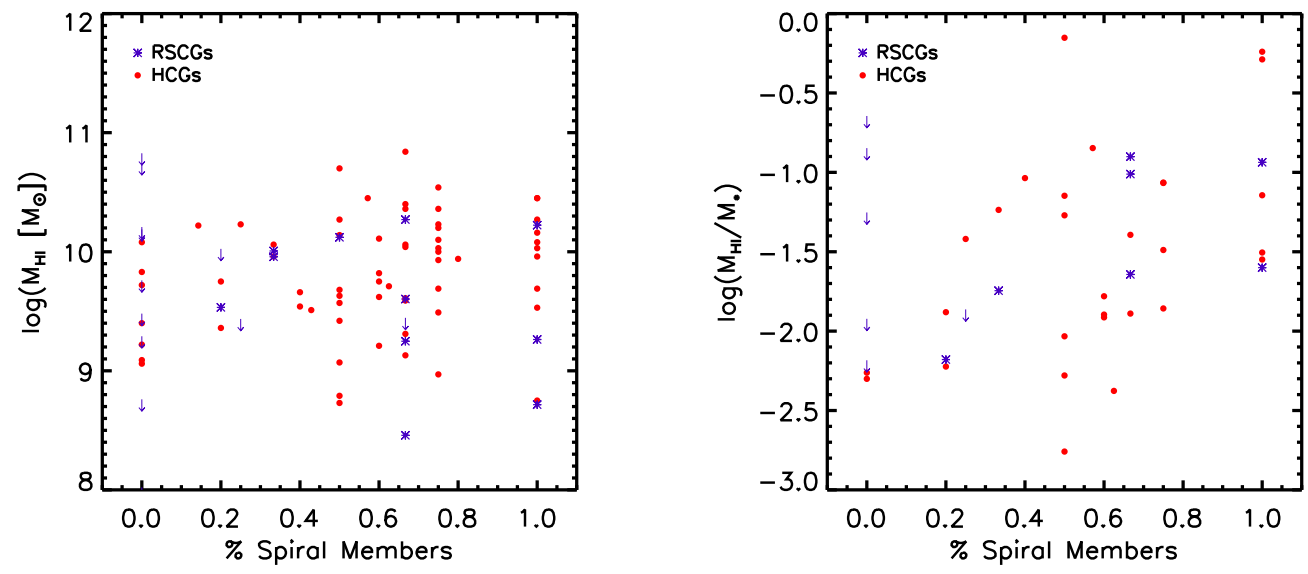

Fig. 5.4.- Left: $\log \left(M_{H I}\right)$ as a function of spiral fraction. Right: $\log \left(\frac{M_{H I}}{M_{*}}\right)$ as a function of spiral fraction. In both plots, the red circles are HCGs, purple asterisks are RSCGs. Clearly, there is no significant correspondance between $M_{H_{I}}$ and spiral fraction. However, $\log \left(\frac{M_{H I}}{M_{*}}\right)$ does increase with increasing spiral fraction, albeit with large scatter. 
et al. 2004).

\subsection{Conclusions}

The H I properties of our detected RSCGs are similar to those of the HCGs. For all groups, stellar mass is larger than H I mass with an average H I content of $\log \left(\frac{M_{H I}}{M_{*}}\right)=-1.51 \pm 0.62$. Groups with a larger fraction of ellipticals or lenticulars, on average, lower H I content compared to groups with mostly spirals. Not surprisingly, galaxies in H I-rich compact groups occupy the optical CMD similarly to low-density environments, where galaxies tend to have a larger reservoir of gas. Meanwhile, galaxies in H I-poor compact groups predominantly fall on the red sequence - similar to high-density environments - where galaxies tend to be depleted of cold gas.

As Figure 5.1 shows, it is impossible with this data to try and get a handle on the kinematics in these groups or determine the distribution of $\mathrm{H} \mathrm{I}$ - we do not know whether the H I is in the galaxies or the intragroup medium. Furthermore, the only way to determine H I masses for the seven groups with strong radio continuum emission in or near the beam is to image them with an interferometer. To truly understand what is happening to the $\mathrm{H} \mathrm{I}$, we need to map its distribution and compare this with the star formation (from Tzanavaris et al. 2010; Lenkic et al. in prep) and stellar populations of the member galaxies. 

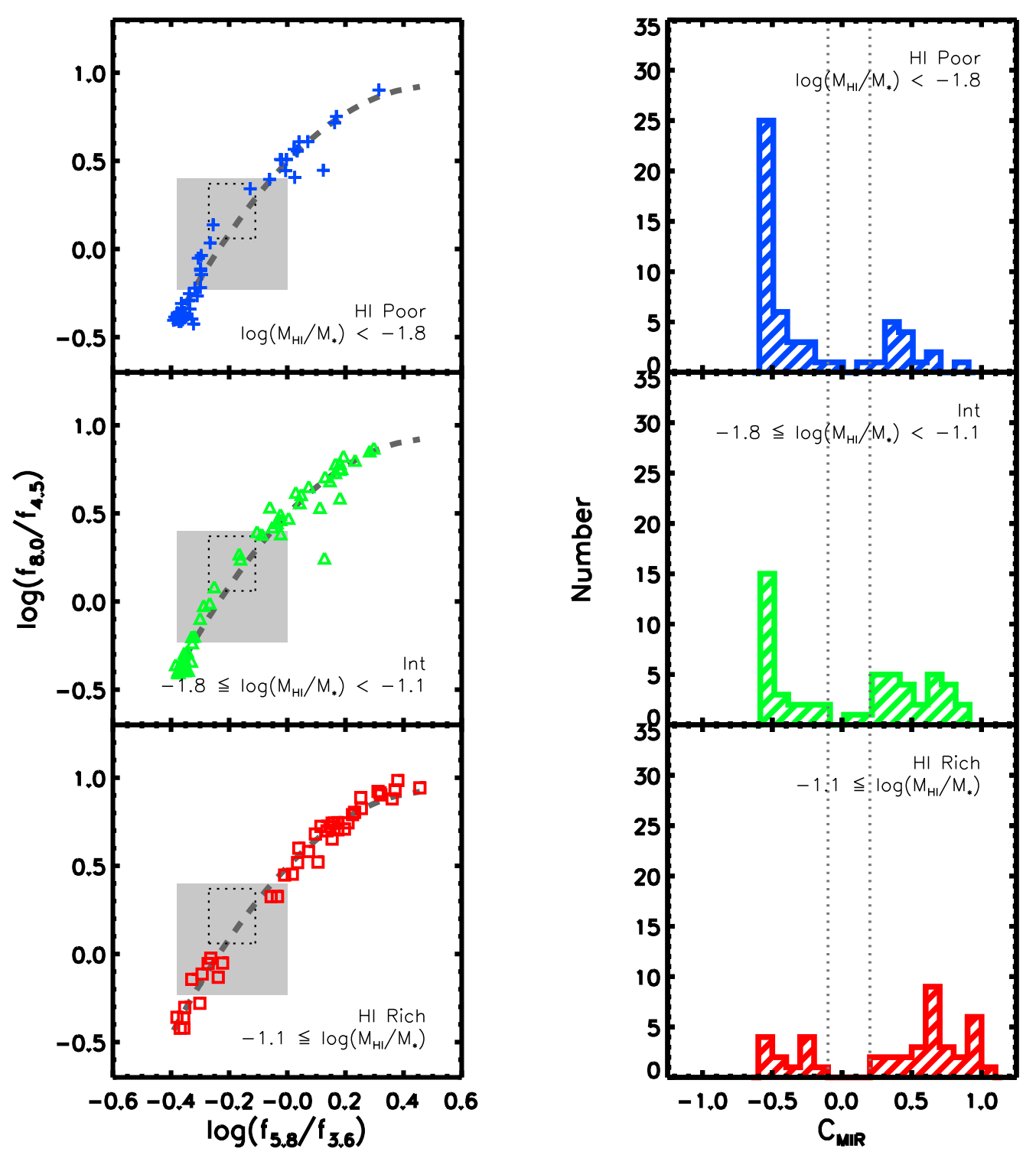

Fig. 5.5.- Left: The distribution of galaxies in IRAC colorspace, broken up by group H I content. Right: Histograms of H I color along the dashed line. There is a clear correlation between group H I content and galaxy mid-IR color - a larger fraction of galaxies in H I-rich groups have colors indicative of activity. 

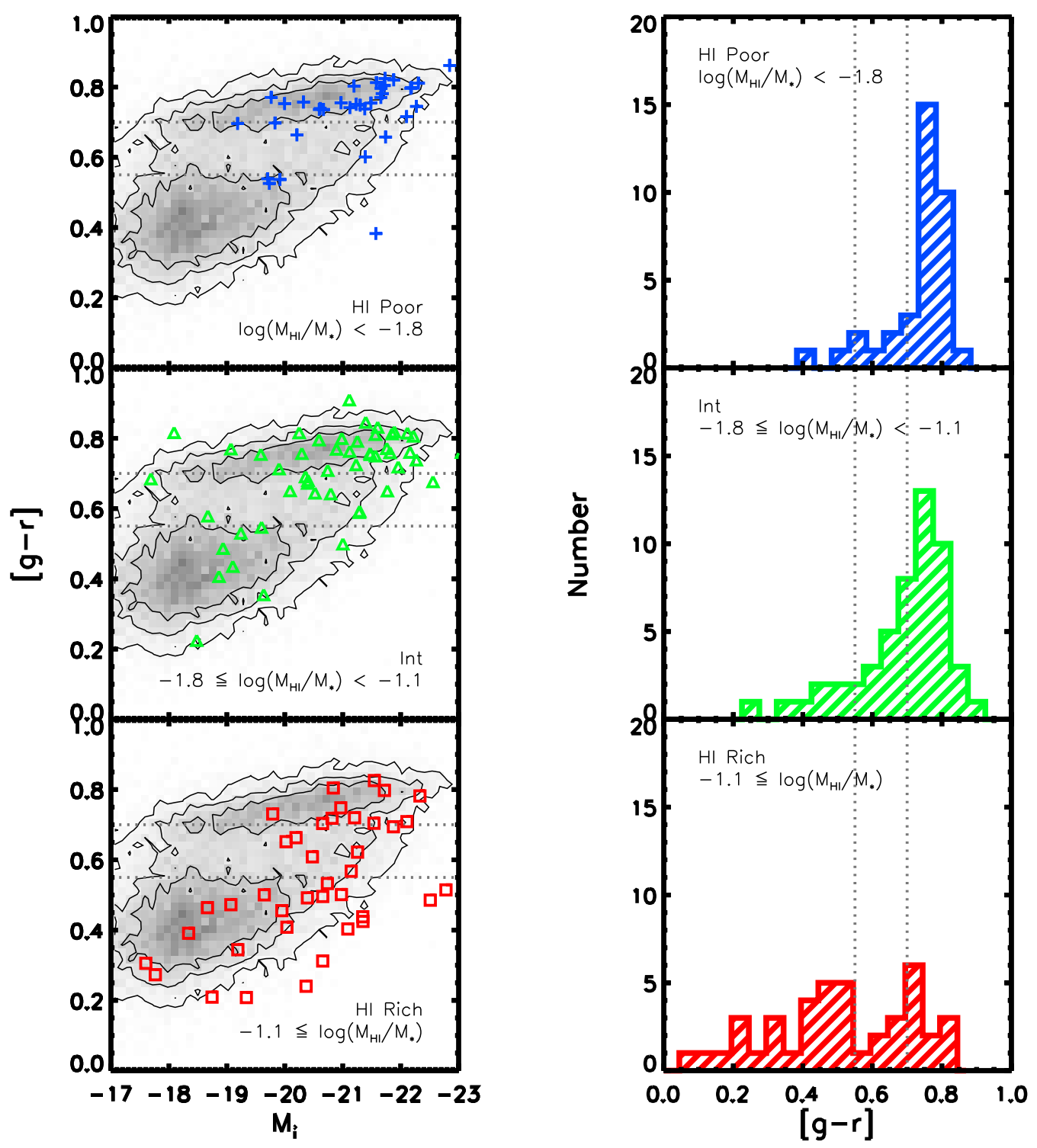

Fig. 5.6. - Left: The distribution of galaxies in the optical CMD, broken up by group H I content. Right: Histograms of optical color. Like the mid-IR colors, there is a correlation between group $\mathrm{H}$ I content and galaxy optical color. This is reminiscent of how the distribution of galaxies in a CMD changes with galaxy environment. 
Chapter 6

Summary and Future Work 
This thesis has explored the effect of the compact group environment on galaxy evolution. I have found that the frequent and prolonged gravitational encounters that occur in compact groups affect the evolution of the constituent galaxies in a myriad of ways, for example gas processing and star formation.

Chapter 2 explores the impact of this environment in detail by examining the distribution in mid-infrared (mid-IR: $3.6-8.0 \mu \mathrm{m}$ ) IRAC colorspace of 42 galaxies from 12 Hickson compact groups in comparison with several control samples, including the LVL+SINGS galaxies, interacting galaxies, and galaxies from the Coma Cluster. This study reveals that the HCG galaxies are strongly bimodal, with statistically significant evidence for a gap in their distribution. In contrast, none of the other samples show such a marked gap, and only galaxies in the Coma infall region have a distribution that is statistically consistent with the HCGs in this parameter space. To further investigate the cause of the HCG gap, this work compares the galaxy morphologies of the HCG and LVL+SINGS galaxies, and also probe the specific star formation rate (SSFR) of the HCG galaxies. While galaxy morphology in HCG galaxies is strongly linked to position with mid-IR colorspace, the more fundamental property appears to be the SSFR, or star formation rate normalized by stellar mass. This work concludes that the unusual mid-IR color distribution of HCG galaxies is a direct product of their environment, which is most similar to that of the Coma infall region. In both cases, galaxy densities are high, but gas has not been fully processed or stripped. This yields speculation that the compact group environment fosters accelerated evolution of galaxies from star-forming and neutral gas-rich to quiescent and neutral gas-poor, leaving few members in the mid-IR gap at any time.

Chapter 3 investigates the origin and nature of this gap by compiling a larger sample of 37 compact groups in addition to the original 12 groups studied by Johnson 
et al. (2007) (yielding 174 individual galaxies with reliable mid-IR photometry). This study finds that a statistically significant deficit of galaxies in this gap region of mid-IR colorspace is persistant in the full sample, lending support to the hypothesis that the compact group environment inhibits moderate SSFRs. Using this expanded sample, this work has more fully characterized the distribution of galaxies in this colorspace and quantified the low density region more fully with respect to midIR bluer and mid-IR redder colors. This investigation reveals a curvature in the colorspace distribution, which is fully consistent with increasing dust temperature as the activity in a galaxy increases. This full sample of 49 compact groups allows subdivision of the data according to physical properties of the groups. An analysis of these subsamples indicates that neither projected physical diameter nor density show a trend in colorspace within the values represented by this sample. The apparent lack of a trend is hypothesized to be due to the relatively small range of properties in this sample, whose groups have already been pre-selected to be compact and dense. Thus, the relative influence of stochastic effects (such as the particular distribution and amount of star formation in individual galaxies) becomes dominant. Analysis of the spectral energy distributions of member galaxies as a function of their location in colorspace shows that galaxies in different regions of mid-IR colorspace contain dust with varying temperatures and/or polycyclic aromatic hydrocarbon (PAH) emission.

Though compact group galaxies in the mid-IR dearth are transition galaxies between actively star forming and quiescent, chapter 4 finds that they have already transitioned to the optical $([\mathrm{g}-\mathrm{r}])$ red sequence. This work investigates the optical color-magnitude diagram (CMD) of 99 compact groups containing 348 galaxies and compare the optical CMD with mid-IR color space for compact group galaxies. Redshifts available from SDSS reveals new galaxy members for 6 groups. By combining 
optical and mid-IR data, this survey obtains information on both the dust and the stellar populations in compact group galaxies. This research also compares with more isolated galaxies and galaxies in the Coma cluster, revealing that, similar to clusters, compact groups are dominated by optically red galaxies. While compact group transition galaxies lie on the optical red sequence, LVL+SINGS mid-IR transition galaxies span the range of optical colors. The dearth of mid-IR transition galaxies in compact groups may be due to a lack of moderately star forming low mass galaxies; the relative lack of these galaxies could be due to their relatively small gravitational potential wells. This makes them more susceptible to this dynamic environment, thus causing them to more easily lose gas or be accreted by larger members.

To understand how the compact group environment affects galaxy evolution, it is important to study the gas and dust processes in these groups. Chapter 5 presents single-dish neutral hydrogen (H I) observations of compact groups, which allow measurement of the H I mass of each group in the sample. This study compares the H I-to-stellar mass ratio with mid-IR indicators of star formation and optical $[g-r]$ color to search for correlations between gas content and star formation activity. This work finds that quiescent galaxies tend to live in H I-poor groups, and galaxies with active star formation are more commonly found in H I-rich groups regardless of the location and distribution of the H I gas, though there are "rogue" galaxies whose star formation does not correlate with group H I content. Ultimately, characterizing how the gas is processed in compact groups and the processing timescales will improve our understanding of gas processing and the interstellar medium in environments similar to that in the earlier Universe.

This work has helped further our understanding of gas processing and galaxy evolution in compact groups, which is directly relevant to how our universe evolved to its 
current state. The mechanisms at work in compact groups are the same mechanisms at work in the earlier universe. The gas processing and star formation in compact groups reveals how these processes occur under the conditions of heierachical structure formation.

\subsection{Outstanding Questions}

Gas processing in the earlier universe is a crucial piece in the galaxy formation puzzle. Compact groups offer nearby sites undergoing these critical processes, but the effect of constant interactions in these groups is not yet fully understood. Although there are many factors affecting the galaxies and even the groups themselves, key evidence suggests that the interstellar/intragroup medium is a primary driver of galaxy evolution in these groups. The crux of this research is answering two intertwined questions: 1) how have the high densities and multiple interactions affected the gas, and 2) how does the gas distribution affect the evolution of the galaxies? As a local analog to earlier universe conditions, understanding these processes in compact groups will bring us much closer to solving this puzzle. The range in compact group properties will distinguish which and how different environmental parameters impact galaxy evolution in groups. This will reveal the effect of frequent and prolonged interactions on star formation and gas processing in this environment conducive to hierarchical merging.

Disentangling the physics in compact groups through comparing stellar populations, gas distributions, and dust properties will help answer:

\section{How is galaxy evolution related to group gas content?}

We must determine the state of the gas in these groups and compare with the properties of the constituent galaxies. The distribution and excitation of the molecular 
gas will reveal to what extent it has been disturbed by the compact group environment. Momose et al. (2013) see enhancement of star formation in regions of high gas density by non-linear processes; this should also be the case in gas-rich compact groups. I will compare the star formation and the molecular gas distributions for gas-rich and gas-poor groups. The gas processing sequence of Verdes-Montenegro et al. (2001) implies that H I-rich groups are less processed and have more latetype galaxies and less intragroup diffuse X-ray emission, while H I-poor groups have finished forming stars, and most of their gas is now found in the hot plasma of the intragroup medium. Ponman et al. (1996) claim that a steepening in the $L_{X}-T$ relation for compact groups compared to clusters may be linked to galaxyscale phenomena such as winds, though Desjardins et al. (submitted) find that some high-temperature compact groups do agree with the cluster relation. If we hope to understand how the gas is processed in compact groups, we must have a complete census of not only where the gas is, but also its state. Only then can we begin to determine the effect of this high density environment where interactions are prevalent.

\section{What are the physical properties of galaxies along the mid-IR sequence?}

The mid-IR transition region reveals that something very unique is happening in the environment of compact groups and in the outer regions of clusters. To determine exactly how these galaxies evolve differently, we must probe the detailed physics of galaxies in compact groups. To do this, we must obtain spectra of these galaxies, as spectra will reveal the differences in stellar populations, as well as gas and dust properties in galaxies with different mid-IR colors. Cluver et al. (2013) has already made great progress, finding enhanced $\mathrm{H}_{2}$ excitation in compact group galaxies in or near the mid-IR canyon, evidence that changing gas properties 
rapidly extinguish star formation. However, their study was constrained by small sample size and inefficiently placed apertures due to the observational limitations caused by Spitzer's roll angles. To truly understand the changes occuring in these galaxies, we need a comprehensive study of compact group galaxies along the midIR sequence, in the mid-IR and beyond. 
Appendix A

Optical Photometry 
Tables A.1 and A.2 present the apparent magnitudes of the sample, along with alternate names for the RSCG galaxies. 
Table A.1. Photometry for HCG Galaxies

\begin{tabular}{cccc}
\hline \hline Galaxy & g' & r' & i' \\
& mag & mag & mag \\
\hline
\end{tabular}

HCG 1

$\begin{array}{llll}\text { A } & 14.95 \pm 0.02 & 14.38 \pm 0.01 & 14.11 \pm 0.01 \\ \text { B } & 15.16 \pm 0.02 & 14.52 \pm 0.01 & 14.24 \pm 0.01 \\ \text { C } & 16.53 \pm 0.01 & 15.80 \pm 0.01 & 15.44 \pm 0.02 \\ \text { D } & 15.32 \pm 0.01 & 14.50 \pm 0.01 & 14.14 \pm 0.01\end{array}$

HCG 2

$\begin{array}{llll}\mathrm{A} & 13.55 \pm 0.01 & 13.24 \pm 0.01 & 13.17 \pm 0.01 \\ \mathrm{~B} & 14.07 \pm 0.01 & 13.58 \pm 0.01 & 13.43 \pm 0.01 \\ \mathrm{C} & 14.51 \pm 0.01 & 14.05 \pm 0.01 & 13.87 \pm 0.01\end{array}$

HCG 3

$\begin{array}{llll}\text { A } & 14.87 \pm 0.01 & 14.23 \pm 0.01 & 13.94 \pm 0.01 \\ \text { B } & 14.97 \pm 0.01 & 14.15 \pm 0.01 & 13.78 \pm 0.01 \\ \text { D } & 15.18 \pm 0.01 & 14.39 \pm 0.01 & 14.03 \pm 0.01\end{array}$

HCG 4

$\begin{array}{llll}\text { A } & 13.35 \pm 0.01 & 12.86 \pm 0.01 & 12.67 \pm 0.01 \\ \text { B } & 15.20 \pm 0.01 & 14.70 \pm 0.01 & 14.53 \pm 0.01 \\ \text { C } & 15.50 \pm 0.01 & 14.69 \pm 0.01 & 14.35 \pm 0.01 \\ \text { D } & 15.57 \pm 0.01 & 14.96 \pm 0.01 & 14.71 \pm 0.01\end{array}$

HCG $7^{\mathrm{a}}$

$\begin{array}{llll}\text { A } & 13.03 \pm 0.01 & 12.27 \pm 0.01 & 11.95 \pm 0.01\end{array}$

$\begin{array}{llll}\text { B } & 13.77 \pm 0.01 & 13.00 \pm 0.01 & 12.64 \pm 0.01\end{array}$

$\begin{array}{llll}\text { C } & 13.24 \pm 0.01 & 12.65 \pm 0.01 & 12.47 \pm 0.01\end{array}$

D $\quad 14.96 \pm 0.01 \quad 14.41 \pm 0.01 \quad 14.16 \pm 0.01$

HCG $10^{\mathrm{b}}$
A $\quad 12.97 \pm 0.01$
$12.18 \pm 0.01 \quad 11.78 \pm 0.01$
B $\quad 12.88 \pm 0.01$
$12.09 \pm 0.01$
$11.71 \pm 0.01$
C $\quad 14.25 \pm 0.01$
$13.43 \pm 0.01$
$13.03 \pm 0.01$
D $\quad 15.12 \pm 0.01$
$14.42 \pm 0.01$
$14.09 \pm 0.01$ 
Table A.1-Continued

\begin{tabular}{cccc}
\hline \hline Galaxy & g' & r' & i' \\
& mag & mag & mag \\
\hline
\end{tabular}

HCG 14

$\begin{array}{llll}\text { A } & 14.48 \pm 0.01 & 13.70 \pm 0.01 & 13.34 \pm 0.01 \\ \text { B } & 14.08 \pm 0.01 & 13.33 \pm 0.01 & 13.00 \pm 0.01 \\ \text { C } & 15.68 \pm 0.01 & 14.95 \pm 0.01 & 14.67 \pm 0.01\end{array}$

HCG 15

$\begin{array}{llll}\text { A } & 14.13 \pm 0.01 & 13.31 \pm 0.01 & 12.91 \pm 0.01 \\ \mathrm{~B} & 14.25 \pm 0.01 & 13.48 \pm 0.01 & 13.13 \pm 0.01 \\ \mathrm{C} & 14.25 \pm 0.01 & 13.47 \pm 0.01 & 13.11 \pm 0.01 \\ \mathrm{D} & 14.59 \pm 0.01 & 13.84 \pm 0.01 & 13.49 \pm 0.01 \\ \mathrm{E} & 14.90 \pm 0.01 & 14.15 \pm 0.01 & 13.82 \pm 0.01 \\ \mathrm{~F} & 15.85 \pm 0.01 & 15.32 \pm 0.01 & 15.09 \pm 0.02\end{array}$

HCG $16^{\mathrm{c}}$

$\begin{array}{llll}\text { A } & 12.54 \pm 0.01 & 11.83 \pm 0.01 & 11.49 \pm 0.01 \\ \mathrm{~B} & 13.26 \pm 0.01 & 12.44 \pm 0.01 & 12.05 \pm 0.01 \\ \mathrm{C} & 13.20 \pm 0.01 & 12.57 \pm 0.01 & 12.34 \pm 0.01\end{array}$

HCG $22^{\text {d }}$

$\begin{array}{llll}\text { A } & 12.30 \pm 0.01 & 11.50 \pm 0.01 & 11.11 \pm 0.01 \\ \text { B } & 14.60 \pm 0.01 & 13.91 \pm 0.01 & 13.58 \pm 0.01 \\ \text { C } & 13.79 \pm 0.01 & 13.26 \pm 0.01 & 13.04 \pm 0.01\end{array}$

HCG 25

$\begin{array}{llll}\text { A } & 14.10 \pm 0.01 & 13.70 \pm 0.01 & 13.55 \pm 0.01 \\ \text { B } & 14.10 \pm 0.02 & 13.30 \pm 0.01 & 12.91 \pm 0.01 \\ \text { D } & 15.53 \pm 0.01 & 14.88 \pm 0.01 & 14.61 \pm 0.01 \\ \text { F } & 15.88 \pm 0.02 & 15.15 \pm 0.01 & 14.84 \pm 0.01\end{array}$

HCG 31

$\begin{array}{cccc}\mathrm{AC} & 13.52 \pm 0.01 & 13.28 \pm 0.01 & 13.32 \pm 0.01 \\ \mathrm{~B} & 15.17 \pm 0.01 & 14.96 \pm 0.01 & 14.94 \pm 0.01 \\ \mathrm{E} & 17.15 \pm 0.02 & 17.00 \pm 0.02 & 17.18 \pm 0.02\end{array}$


Table A.1-Continued

\begin{tabular}{cccc}
\hline \hline Galaxy & $\begin{array}{c}\text { g' } \\
\text { mag }\end{array}$ & r' & $\begin{array}{c}\text { i' } \\
\text { mag }\end{array}$ \\
\hline F & $17.25 \pm 0.02$ & $17.40 \pm 0.02$ & $17.41 \pm 0.03$ \\
G & $14.59 \pm 0.01$ & $14.38 \pm 0.01$ & $14.35 \pm 0.01$ \\
Q & $16.48 \pm 0.01$ & $16.18 \pm 0.01$ & $16.08 \pm 0.01$ \\
HCG 37 & & & \\
A & $12.96 \pm 0.01$ & $12.20 \pm 0.01$ & $11.85 \pm 0.01$ \\
B & $14.52 \pm 0.01$ & $13.69 \pm 0.01$ & $13.30 \pm 0.01$ \\
C & $15.71 \pm 0.03$ & $14.95 \pm 0.03$ & $14.61 \pm 0.03$ \\
D & $16.39 \pm 0.01$ & $15.86 \pm 0.01$ & $15.66 \pm 0.01$ \\
E & $16.40 \pm 0.01$ & $15.64 \pm 0.01$ & $15.31 \pm 0.01$
\end{tabular}

HCG 38
A
$15.40 \pm 0.01$
$14.60 \pm 0.01$
$14.22 \pm 0.01$
B $\quad 14.67 \pm 0.01$
$14.02 \pm 0.01$
$13.70 \pm 0.01$
C $\quad 15.76 \pm 0.02$
$15.05 \pm 0.02$
$14.73 \pm 0.01$

HCG 43

$\begin{array}{llll}\text { A } & 15.36 \pm 0.01 & 14.63 \pm 0.01 & 14.30 \pm 0.01 \\ \text { B } & 15.76 \pm 0.01 & 15.16 \pm 0.01 & 14.90 \pm 0.01 \\ \text { C } & 15.52 \pm 0.01 & 14.73 \pm 0.01 & 14.38 \pm 0.01 \\ \text { D } & 16.71 \pm 0.01 & 16.05 \pm 0.01 & 15.77 \pm 0.01 \\ \text { E } & 16.80 \pm 0.01 & 15.92 \pm 0.01 & 15.54 \pm 0.01\end{array}$

$\mathrm{HCG} 44^{\mathrm{e}}$
A
$11.34 \pm 0.01$
$10.57 \pm 0.01$
$10.16 \pm 0.01$
B $\quad 11.49 \pm 0.01$
$10.74 \pm 0.01$
$10.35 \pm 0.01$
C $\quad 12.60 \pm 0.01$
$11.91 \pm 0.01$
$11.56 \pm 0.01$
D $\quad 13.40 \pm 0.01$
$12.97 \pm 0.01$
$12.82 \pm 0.01$

HCG 46
A
$15.48 \pm 0.01$
$14.68 \pm 0.01$
$14.32 \pm 0.01$
B $\quad 15.49 \pm 0.01$
$14.72 \pm 0.01 \quad 14.34 \pm 0.01$
C
$15.47 \pm 0.01$
$14.68 \pm 0.01$
$14.32 \pm 0.01$ 
Table A.1—Continued

\begin{tabular}{cccc}
\hline \hline Galaxy & $\begin{array}{c}\text { g' } \\
\text { mag }\end{array}$ & $\begin{array}{c}\text { r' } \\
\text { mag }\end{array}$ & $\begin{array}{c}\text { i' } \\
\text { mag }\end{array}$ \\
\hline D & $15.69 \pm 0.01$ & $14.90 \pm 0.01$ & $14.56 \pm 0.01$ \\
HCG 47 & & & \\
A & $14.10 \pm 0.01$ & $13.42 \pm 0.01$ & $13.11 \pm 0.01$ \\
B & $14.97 \pm 0.01$ & $14.15 \pm 0.01$ & $13.78 \pm 0.01$ \\
C & $16.22 \pm 0.01$ & $15.54 \pm 0.01$ & $15.27 \pm 0.01$ \\
D & $15.79 \pm 0.01$ & $15.14 \pm 0.01$ & $14.87 \pm 0.01$
\end{tabular}

HCG 49

$\begin{array}{llll}\text { A } & 15.91 \pm 0.01 & 15.59 \pm 0.01 & 15.50 \pm 0.01 \\ \text { B } & 16.42 \pm 0.01 & 16.28 \pm 0.01 & 16.28 \pm 0.01 \\ \text { C } & 17.25 \pm 0.01 & 16.99 \pm 0.01 & 16.96 \pm 0.01 \\ \text { D } & 17.00 \pm 0.01 & 16.72 \pm 0.01 & 16.67 \pm 0.01\end{array}$

HCG 51

$\begin{array}{llll}\text { A } & 14.33 \pm 0.01 & 13.51 \pm 0.01 & 13.14 \pm 0.01 \\ \text { B } & 14.89 \pm 0.01 & 14.13 \pm 0.01 & 13.78 \pm 0.01 \\ \text { C } & 14.22 \pm 0.01 & 13.41 \pm 0.01 & 13.04 \pm 0.01 \\ \text { D } & 15.47 \pm 0.01 & 14.70 \pm 0.01 & 14.37 \pm 0.01 \\ \text { E } & 15.10 \pm 0.01 & 14.25 \pm 0.01 & 13.86 \pm 0.01 \\ \text { F } & 15.80 \pm 0.01 & 15.01 \pm 0.01 & 14.67 \pm 0.01 \\ \text { G } & 16.18 \pm 0.02 & 15.36 \pm 0.02 & 15.01 \pm 0.02\end{array}$

HCG 53

$\begin{array}{llll}\text { A } & 13.39 \pm 0.01 & 12.78 \pm 0.01 & 12.50 \pm 0.01 \\ \text { B } & 14.51 \pm 0.01 & 13.72 \pm 0.01 & 13.37 \pm 0.01 \\ \text { C } & 14.87 \pm 0.01 & 14.43 \pm 0.01 & 14.26 \pm 0.01\end{array}$

HCG 54
$\begin{array}{llll}\text { A } & 14.86 \pm 0.01 & 14.59 \pm 0.01 & 14.48 \pm 0.01\end{array}$
$\begin{array}{llll}\text { B } & 16.34 \pm 0.02 & 16.18 \pm 0.02 & 16.26 \pm 0.03\end{array}$
$\begin{array}{llll}\text { C } & 16.77 \pm 0.01 & 16.68 \pm 0.01 & 16.70 \pm 0.01\end{array}$

HCG 56 
Table A.1—Continued

\begin{tabular}{cccc}
\hline \hline Galaxy & g' & r' & i' \\
& mag & mag & mag \\
\hline A & $15.76 \pm 0.01$ & $15.02 \pm 0.01$ & $14.65 \pm 0.01$ \\
B & $14.51 \pm 0.01$ & $13.85 \pm 0.01$ & $13.58 \pm 0.01$ \\
C & $15.25 \pm 0.01$ & $14.51 \pm 0.01$ & $14.20 \pm 0.01$ \\
D & $16.11 \pm 0.01$ & $15.35 \pm 0.01$ & $15.00 \pm 0.01$ \\
E & $16.14 \pm 0.01$ & $15.61 \pm 0.01$ & $15.41 \pm 0.01$ \\
HCG $57^{f}$ & & & \\
A & $14.02 \pm 0.01$ & $13.16 \pm 0.01$ & $12.75 \pm 0.01$ \\
B & $14.39 \pm 0.01$ & $13.65 \pm 0.01$ & $13.33 \pm 0.01$ \\
C & $14.57 \pm 0.01$ & $13.77 \pm 0.01$ & $13.42 \pm 0.01$ \\
D & $15.05 \pm 0.01$ & $14.45 \pm 0.01$ & $14.21 \pm 0.01$ \\
E & $15.05 \pm 0.01$ & $14.23 \pm 0.01$ & $13.87 \pm 0.01$ \\
F & $15.19 \pm 0.01$ & $14.43 \pm 0.01$ & $14.11 \pm 0.01$ \\
G & $15.55 \pm 0.01$ & $14.74 \pm 0.01$ & $14.40 \pm 0.01$ \\
H & $16.66 \pm 0.01$ & $15.90 \pm 0.01$ & $15.60 \pm 0.01$ \\
HCG 58 & & & \\
A & $13.41 \pm 0.01$ & $12.69 \pm 0.01$ & $12.38 \pm 0.01$ \\
B & $13.18 \pm 0.01$ & $12.44 \pm 0.01$ & $12.11 \pm 0.01$ \\
C & $13.78 \pm 0.01$ & $13.05 \pm 0.01$ & $12.72 \pm 0.01$ \\
D & $14.20 \pm 0.01$ & $13.41 \pm 0.01$ & $13.05 \pm 0.01$ \\
E & $14.80 \pm 0.01$ & $14.27 \pm 0.01$ & $14.05 \pm 0.01$ \\
HCG 59g & & & \\
A & $14.39 \pm 0.01$ & $13.74 \pm 0.01$ & $13.47 \pm 0.01$ \\
B & $15.13 \pm 0.01$ & $14.42 \pm 0.01$ & $14.09 \pm 0.01$ \\
C & $15.76 \pm 0.01$ & $15.27 \pm 0.01$ & $15.06 \pm 0.01$ \\
D & $15.69 \pm 0.01$ & $15.46 \pm 0.01$ & $15.51 \pm 0.01$ \\
HCG $61^{h}$ & & & \\
A & $12.80 \pm 0.01$ & $12.03 \pm 0.01$ & $11.65 \pm 0.01$ \\
C & $13.86 \pm 0.01$ & $13.04 \pm 0.01$ & $12.63 \pm 0.01$ \\
& & &
\end{tabular}


Table A.1-Continued

\begin{tabular}{cccc}
\hline \hline Galaxy & $\begin{array}{c}\text { g' } \\
\text { mag }\end{array}$ & $\begin{array}{c}\text { r' } \\
\text { mag }\end{array}$ & $\begin{array}{c}\text { i' } \\
\text { mag }\end{array}$ \\
\hline D & $14.01 \pm 0.01$ & $13.33 \pm 0.01$ & $13.03 \pm 0.01$ \\
HCG 68 & & & \\
D & $13.82 \pm 0.01$ & $13.16 \pm 0.01$ & $12.84 \pm 0.01$ \\
E & $14.26 \pm 0.01$ & $13.56 \pm 0.01$ & $13.22 \pm 0.01$ \\
HCG 69 & & & \\
A & $14.98 \pm 0.01$ & $14.23 \pm 0.01$ & $13.85 \pm 0.01$ \\
B & $15.30 \pm 0.01$ & $14.63 \pm 0.01$ & $14.35 \pm 0.01$ \\
C & $15.57 \pm 0.01$ & $14.82 \pm 0.01$ & $14.51 \pm 0.01$ \\
D & $16.59 \pm 0.01$ & $15.81 \pm 0.01$ & $15.48 \pm 0.01$
\end{tabular}

HCG 71

$\begin{array}{llll}\text { A } & 14.04 \pm 0.01 & 13.39 \pm 0.01 & 13.09 \pm 0.01 \\ \text { B } & 15.03 \pm 0.01 & 14.40 \pm 0.01 & 14.13 \pm 0.01 \\ \text { C } & 15.97 \pm 0.01 & 15.51 \pm 0.01 & 15.34 \pm 0.01\end{array}$

HCG 76

$\begin{array}{llll}\text { A } & 15.06 \pm 0.01 & 14.22 \pm 0.01 & 13.84 \pm 0.01 \\ \text { B } & 14.73 \pm 0.01 & 13.93 \pm 0.01 & 13.57 \pm 0.01 \\ \text { C } & 14.68 \pm 0.01 & 13.88 \pm 0.01 & 13.51 \pm 0.01 \\ \text { D } & 15.11 \pm 0.01 & 14.33 \pm 0.01 & 13.98 \pm 0.01 \\ \text { E } & 16.57 \pm 0.01 & 15.82 \pm 0.01 & 15.50 \pm 0.01 \\ \text { F } & 16.58 \pm 0.01 & 15.82 \pm 0.01 & 15.48 \pm 0.01 \\ \text { G } & 17.10 \pm 0.01 & 16.32 \pm 0.01 & 15.94 \pm 0.01\end{array}$

HCG $79^{j}$
$\begin{array}{llll}\text { A } & 14.37 \pm 0.01 & 13.57 \pm 0.01 & 13.18 \pm 0.01\end{array}$
$\begin{array}{llll}\text { B } & 13.99 \pm 0.01 & 13.27 \pm 0.01 & 12.93 \pm 0.01\end{array}$
$\begin{array}{llll}\text { C } & 15.03 \pm 0.02 & 14.38 \pm 0.02 & 14.07 \pm 0.02\end{array}$
D $\quad 15.87 \pm 0.01 \quad 15.47 \pm 0.01 \quad 15.30 \pm 0.01$

HCG 88

A $\quad 13.48 \pm 0.02 \quad 12.74 \pm 0.01 \quad 12.38 \pm 0.01$ 
Table A.1-Continued

\begin{tabular}{cccc}
\hline \hline Galaxy & g' & r' & i' \\
& mag & mag & mag \\
\hline B & $13.59 \pm 0.02$ & $12.88 \pm 0.01$ & $12.56 \pm 0.01$ \\
C & $14.33 \pm 0.02$ & $13.80 \pm 0.01$ & $13.59 \pm 0.01$ \\
D & $15.27 \pm 0.02$ & $14.70 \pm 0.01$ & $14.45 \pm 0.01$ \\
E $^{\dagger}$ & $17.88 \pm 0.02$ & $17.44 \pm 0.01$ & $17.30 \pm 0.01$ \\
HCG 89 & & & \\
A & $14.86 \pm 0.02$ & $14.38 \pm 0.01$ & $14.22 \pm 0.01$ \\
B & $15.54 \pm 0.02$ & $15.11 \pm 0.01$ & $14.98 \pm 0.01$ \\
C & $16.06 \pm 0.02$ & $15.56 \pm 0.01$ & $15.38 \pm 0.01$ \\
D & $16.62 \pm 0.02$ & $16.39 \pm 0.01$ & $16.37 \pm 0.01$ \\
HCG 92 & & & \\
B & $13.81 \pm 0.02$ & $13.10 \pm 0.02$ & $12.78 \pm 0.02$ \\
C & $13.65 \pm 0.02$ & $12.89 \pm 0.01$ & $12.57 \pm 0.01$ \\
D & $13.55 \pm 0.01$ & $12.81 \pm 0.01$ & $12.47 \pm 0.01$ \\
E & $14.37 \pm 0.01$ & $13.56 \pm 0.01$ & $13.17 \pm 0.01$ \\
HCG 93 & & & \\
A & $12.49 \pm 0.03$ & $11.69 \pm 0.02$ & $11.31 \pm 0.02$ \\
B & $13.22 \pm 0.03$ & $12.62 \pm 0.02$ & $12.35 \pm 0.02$ \\
C & $13.76 \pm 0.03$ & $12.93 \pm 0.02$ & $12.56 \pm 0.02$ \\
D & $14.68 \pm 0.03$ & $13.84 \pm 0.02$ & $13.46 \pm 0.02$ \\
HCG 96 & & & \\
A & $13.33 \pm 0.01$ & $12.82 \pm 0.01$ & $12.59 \pm 0.01$ \\
B & $14.17 \pm 0.01$ & $13.38 \pm 0.01$ & $13.04 \pm 0.01$ \\
C & $15.21 \pm 0.01$ & $14.49 \pm 0.01$ & $14.16 \pm 0.01$ \\
D & $16.62 \pm 0.01$ & $16.28 \pm 0.01$ & $16.18 \pm 0.01$ \\
HCG 97 & & & \\
A & $13.71 \pm 0.01$ & $12.91 \pm 0.01$ & $12.54 \pm 0.01$ \\
B & $15.22 \pm 0.01$ & $14.49 \pm 0.01$ & $14.13 \pm 0.01$ \\
C & $14.41 \pm 0.01$ & $13.68 \pm 0.01$ & $13.35 \pm 0.01$
\end{tabular}


Table A.1-Continued

\begin{tabular}{cccc}
\hline \hline Galaxy & $\begin{array}{c}\text { g' } \\
\text { mag }\end{array}$ & $\begin{array}{c}\text { r' } \\
\text { mag }\end{array}$ & $\begin{array}{c}\text { i' } \\
\text { mag }\end{array}$ \\
\hline D & $14.37 \pm 0.01$ & $13.55 \pm 0.01$ & $13.15 \pm 0.01$ \\
E & $16.08 \pm 0.01$ & $15.31 \pm 0.01$ & $14.96 \pm 0.01$ \\
HCG 98 & & & \\
A & $13.54 \pm 0.01$ & $12.73 \pm 0.01$ & $12.35 \pm 0.01$ \\
B & $14.53 \pm 0.01$ & $13.72 \pm 0.01$ & $13.34 \pm 0.01$ \\
C & $15.83 \pm 0.01$ & $15.06 \pm 0.01$ & $14.72 \pm 0.01$ \\
HCG 99 & & & \\
A & $14.22 \pm 0.01$ & $13.41 \pm 0.01$ & $12.98 \pm 0.01$ \\
B & $14.11 \pm 0.01$ & $13.30 \pm 0.01$ & $12.94 \pm 0.01$ \\
C & $14.99 \pm 0.01$ & $14.21 \pm 0.01$ & $13.86 \pm 0.01$ \\
D & $16.52 \pm 0.01$ & $15.75 \pm 0.01$ & $15.41 \pm 0.01$ \\
E & $16.94 \pm 0.01$ & $16.18 \pm 0.01$ & $15.84 \pm 0.01$ \\
HCG 100 & & & \\
A & $13.41 \pm 0.01$ & $12.71 \pm 0.01$ & $12.41 \pm 0.01$ \\
B & $14.79 \pm 0.01$ & $14.38 \pm 0.01$ & $14.25 \pm 0.01$ \\
C & $15.34 \pm 0.01$ & $14.84 \pm 0.01$ & $14.64 \pm 0.01$ \\
D & $16.23 \pm 0.01$ & $15.77 \pm 0.01$ & $15.62 \pm 0.01$ \\
\hline
\end{tabular}

Note. - ${ }^{\mathrm{a}} \mathrm{RSCG} 3$ 3. ${ }^{\mathrm{b}} \mathrm{RSCG} 12 .{ }^{\mathrm{c}} \mathrm{RSCG} 19 .{ }^{\mathrm{d}} \mathrm{RSCG}$ 20. ${ }^{\mathrm{e}} \mathrm{RSCG}$ 35. ${ }^{\mathrm{f}} \mathrm{RSCG} 43 .{ }^{\mathrm{g}} \mathrm{RSCG} 46 .{ }^{\mathrm{h}} \mathrm{RSCG}$ 49. ${ }^{\mathrm{i}} \mathrm{RSCG}$ 71. ${ }^{\mathrm{j}} \mathrm{RSCG} 77 .{ }^{\mathrm{k}} \mathrm{RSCG} 82 .{ }^{\mathrm{l}} \mathrm{RSCG} 87$. ${ }^{\mathrm{m}} \mathrm{RSCG}$ 89. ${ }^{\dagger}$ Galaxy added by us. 
Table A.2. Photometry for RSCG Galaxies

\begin{tabular}{cccc}
\hline \hline Galaxy & g' & r' & i' \\
& mag & mag & mag \\
\hline
\end{tabular}

RSCG 1

$\begin{array}{lcccc}\text { A } & \text { NGC 70 } & 13.04 \pm 0.02 & 12.33 \pm 0.02 & 11.99 \pm 0.01 \\ \text { B } & \text { NGC 68 } & 13.76 \pm 0.02 & 12.96 \pm 0.01 & 12.60 \pm 0.01 \\ \text { C } & \text { NGC 71 } & 13.93 \pm 0.02 & 13.14 \pm 0.02 & 12.77 \pm 0.01 \\ \text { D } & \text { NGC 72 } & 14.10 \pm 0.02 & 13.28 \pm 0.01 & 12.92 \pm 0.01 \\ \text { E } & \text { NGC 72A } & 15.25 \pm 0.02 & 14.44 \pm 0.01 & 14.08 \pm 0.01\end{array}$

RSCG 2

$\begin{array}{lcccc}\text { A } & \text { UGC 335b } & 14.22 \pm 0.01 & 13.45 \pm 0.01 & 13.09 \pm 0.01 \\ \text { B } & \text { UGC 335a } & 14.62 \pm 0.01 & 13.83 \pm 0.01 & 13.48 \pm 0.01 \\ \text { C } & \text { UGC 331 } & 15.00 \pm 0.01 & 14.28 \pm 0.01 & 13.98 \pm 0.01\end{array}$

RSCG 4

$\begin{array}{ccccc}\text { A } & \text { NGC 235B } & 13.69 \pm 0.01 & 12.97 \pm 0.01 & 12.65 \pm 0.01 \\ \text { B } & \text { NGC 235A } & 15.21 \pm 0.01 & 14.47 \pm 0.01 & 14.14 \pm 0.01 \\ \text { C } & \text { NGC 232 } & \ldots & \ldots & \ldots\end{array}$

RSCG 5

$\begin{array}{lllll}\text { A } & \text { NGC 383 } & 12.58 \pm 0.02 & 11.76 \pm 0.02 & 11.34 \pm 0.01 \\ \text { B } & \text { NGC 385 } & 13.58 \pm 0.02 & 12.79 \pm 0.01 & 12.42 \pm 0.01 \\ \text { C } & \text { NGC 384 } & 13.74 \pm 0.02 & 12.95 \pm 0.01 & 12.55 \pm 0.01 \\ \text { D } & \text { NGC 382 } & 13.94 \pm 0.02 & 13.13 \pm 0.02 & 12.73 \pm 0.02 \\ \text { E } & \text { NGC 386 } & 14.85 \pm 0.02 & 14.09 \pm 0.01 & 13.73 \pm 0.01\end{array}$

RSCG 6
A UGC 816
$13.87 \pm 0.01 \quad 13.37 \pm 0.01$
$13.22 \pm 0.01$
B UGC 813
$14.21 \pm 0.01$
$13.68 \pm 0.01$
$13.46 \pm 0.01$
C CGCG 551-011
$14.43 \pm 0.01$
$13.71 \pm 0.01$
$13.38 \pm 0.01$

RSCG 7
A NGC 499
$12.64 \pm 0.01 \quad 11.81 \pm 0.01$
$11.41 \pm 0.01$
B NGC 495
$13.62 \pm 0.01 \quad 12.82 \pm 0.01$
$12.45 \pm 0.01$
C $\quad$ NGC 501
$14.83 \pm 0.01 \quad 14.03 \pm 0.01$
$13.65 \pm 0.01$ 
Table A.2-Continued

\begin{tabular}{cccc}
\hline \hline Galaxy & g' & r' & i' \\
& mag & mag & mag \\
\hline
\end{tabular}

RSCG 8

$\begin{array}{ccccc}\text { A } & \text { NGC 507 } & 12.04 \pm 0.01 & 11.23 \pm 0.01 & 10.83 \pm 0.01 \\ \text { C } & \text { NGC 508 } & 13.93 \pm 0.01 & 13.05 \pm 0.01 & 12.63 \pm 0.01 \\ \text { B } & \text { NGC 504 } & 13.82 \pm 0.01 & 13.01 \pm 0.01 & 12.64 \pm 0.01 \\ \text { D } & \text { IC 1687 } & 14.66 \pm 0.01 & 13.85 \pm 0.01 & 13.48 \pm 0.01 \\ \text { E } & \text { NGC 503 } & 14.75 \pm 0.01 & 13.97 \pm 0.01 & 13.62 \pm 0.01\end{array}$

RSCG 9

$\begin{array}{ccccc}\text { A } & \text { UGC } 978 & 13.60 \pm 0.01 & 12.82 \pm 0.01 & 12.46 \pm 0.01 \\ \text { B } & \text { IC } 107 & 14.29 \pm 0.01 & 13.53 \pm 0.01 & 13.20 \pm 0.01 \\ \text { C } & \text { IC } 1698 & 14.68 \pm 0.01 & 14.18 \pm 0.01 & 14.00 \pm 0.01\end{array}$

RSCG 10

$\begin{array}{lllll}\text { A } & \text { NGC 538 } & 13.86 \pm 0.01 & 13.05 \pm 0.01 & 12.65 \pm 0.01 \\ \text { B } & \text { UGC 996 } & 14.40 \pm 0.01 & 13.64 \pm 0.01 & 13.30 \pm 0.01 \\ \text { C } & \text { UGC 984 } & 14.43 \pm 0.01 & 13.70 \pm 0.01 & 13.36 \pm 0.01\end{array}$

RSCG 11

$\begin{array}{ccccc}\text { A/B } & \text { NGC 547/545 } & 11.98 \pm 0.01 & 11.18 \pm 0.01 & 10.79 \pm 0.01 \\ \text { C } & \text { NGC 541 } & 12.99 \pm 0.01 & 12.21 \pm 0.01 & 11.83 \pm 0.01 \\ \text { D } & \text { NGC 543 } & 14.47 \pm 0.01 & 13.66 \pm 0.01 & 13.28 \pm 0.01 \\ \text { E } & \text { ARK 45 } & 14.87 \pm 0.01 & 14.08 \pm 0.01 & 13.71 \pm 0.01\end{array}$

RSCG 14
A NGC 680
$12.18 \pm 0.02$
$11.41 \pm 0.01$
$11.04 \pm 0.01$
B NGC 678
$12.56 \pm 0.02$
$11.66 \pm 0.01$
$11.20 \pm 0.01$
C IC 1730
$14.85 \pm 0.02$
$14.18 \pm 0.01$
$13.87 \pm 0.01$

RSCG 17
A NGC 741
$12.15 \pm 0.01$
$11.32 \pm 0.01$
$10.92 \pm 0.01$
B ARK 66
$14.09 \pm 0.02$
$13.25 \pm 0.02$
$12.84 \pm 0.01$
C NGC 742
$14.88 \pm 0.01$
$14.05 \pm 0.01$
$13.68 \pm 0.01$

RSCG 18 
Table A.2-Continued

\begin{tabular}{ccccc}
\hline \hline & Galaxy & g' & r' & i' \\
& & mag & mag & mag \\
\hline A & NGC 736 & $12.77 \pm 0.01$ & $11.97 \pm 0.01$ & $11.58 \pm 0.01$ \\
B & NGC 740 & $14.40 \pm 0.01$ & $13.70 \pm 0.01$ & $13.37 \pm 0.01$ \\
C & NGC 738 & $15.22 \pm 0.01$ & $14.44 \pm 0.01$ & $14.09 \pm 0.01$ \\
RSCG 21 & & & \\
A & NGC 1273 & $13.47 \pm 0.02$ & $12.70 \pm 0.01$ & $12.37 \pm 0.01$ \\
B & NGC 1277 & $13.83 \pm 0.02$ & $12.99 \pm 0.01$ & $12.60 \pm 0.01$ \\
C & CGCG 540-101 & $13.84 \pm 0.02$ & $13.00 \pm 0.01$ & $12.61 \pm 0.01$ \\
RSCG 28 & & & \\
A & NGC 2738 & $13.37 \pm 0.01$ & $12.87 \pm 0.01$ & $12.63 \pm 0.01$ \\
B & NGC 2737 & $13.86 \pm 0.01$ & $13.14 \pm 0.01$ & $12.79 \pm 0.01$ \\
C & CGCG 121-011 & $15.09 \pm 0.01$ & $14.73 \pm 0.01$ & $14.58 \pm 0.01$
\end{tabular}

RSCG 30
A NGC 2778
$13.06 \pm 0.01 \quad 12.31 \pm 0.01$
$11.93 \pm 0.01$
B NGC 2780
$13.98 \pm 0.01$
$13.36 \pm 0.01$
$13.06 \pm 0.01$
C $\quad$ NGC 2779
$15.12 \pm 0.01$
$14.50 \pm 0.01$
$14.23 \pm 0.01$

RSCG 31
A NGC 2798
$12.70 \pm 0.01$
$12.00 \pm 0.01$
$11.70 \pm 0.01$
B NGC 2799
$13.94 \pm 0.01$
$13.47 \pm 0.01$
$13.28 \pm 0.01$
C UGC 4904
$14.56 \pm 0.01$
$14.17 \pm 0.01$
$14.01 \pm 0.01$

RSCG 32
A NGC 2832
$12.40 \pm 0.01$
$11.57 \pm 0.01$
$11.17 \pm 0.01$
B NGC 2831
$14.28 \pm 0.02$
$13.46 \pm 0.02$
$13.07 \pm 0.02$
C NGC 2830
$14.56 \pm 0.01$
$13.80 \pm 0.01$
$13.45 \pm 0.01$

RSCG 33
A NGC 2911
$12.40 \pm 0.01$
$11.56 \pm 0.01$
$11.14 \pm 0.01$
B NGC 2914
$13.52 \pm 0.01$
$12.76 \pm 0.01$
$12.38 \pm 0.01$
C UGC 5093
$14.72 \pm 0.01 \quad 14.05 \pm 0.01$
$13.72 \pm 0.01$

RSCG 34 
Table A.2 - Continued

\begin{tabular}{ccccc}
\hline \hline & Galaxy & $\begin{array}{c}\text { g' } \\
\text { mag }\end{array}$ & $\begin{array}{c}\text { r' } \\
\text { mag }\end{array}$ & $\begin{array}{c}\text { i' } \\
\text { mag }\end{array}$ \\
\hline A & NGC 2964 & $11.64 \pm 0.01$ & $11.05 \pm 0.01$ & $10.77 \pm 0.01$ \\
B & NGC 2968 & $12.32 \pm 0.01$ & $11.42 \pm 0.01$ & $10.94 \pm 0.01$ \\
C & NGC 2970 & $14.25 \pm 0.01$ & $13.67 \pm 0.01$ & $13.38 \pm 0.01$
\end{tabular}

RSCG 36

$\begin{array}{ccccc}\text { A } & \text { NGC 3379 } & 9.92 \pm 0.01 & 9.16 \pm 0.01 & 8.76 \pm 0.01 \\ \text { B } & \text { NGC 3384 } & 10.38 \pm 0.01 & 9.64 \pm 0.01 & 9.27 \pm 0.01 \\ \text { C } & \text { NGC 3389 } & 12.03 \pm 0.01 & 11.69 \pm 0.01 & 11.53 \pm 0.01\end{array}$

RSCG 37

$\begin{array}{ccccc}\text { A } & \text { NGC 3377 } & 10.84 \pm 0.01 & 10.16 \pm 0.01 & 9.83 \pm 0.01 \\ \text { B } & \text { NGC 3377A } & 14.04 \pm 0.01 & 13.58 \pm 0.01 & 13.35 \pm 0.01 \\ \text { C } & \text { CGCG 066-026 } & 15.57 \pm 0.01 & 14.96 \pm 0.01 & 14.66 \pm 0.01\end{array}$

RSCG 38

$\begin{array}{lllll}\text { A } & \text { NGC 3430 } & 11.89 \pm 0.01 & 11.39 \pm 0.01 & 11.17 \pm 0.01 \\ \text { B } & \text { NGC 3424 } & 12.81 \pm 0.01 & 12.13 \pm 0.01 & 11.77 \pm 0.01 \\ \text { C } & \text { NGC 3413 } & 13.05 \pm 0.01 & 12.70 \pm 0.01 & 12.54 \pm 0.01\end{array}$

RSCG 39
A NGC 3455
$12.91 \pm 0.01 \quad 12.57 \pm 0.01$
$12.41 \pm 0.01$
B NGC 3454
$13.69 \pm 0.01$
$13.11 \pm 0.01$
$12.83 \pm 0.01$
C UGC 6035
$14.57 \pm 0.01$
$14.23 \pm 0.01$
$14.05 \pm 0.01$
D CGCG 095-070
$15.20 \pm 0.01 \quad 14.80 \pm 0.01$
$14.61 \pm 0.01$

RSCG 40

$\begin{array}{lcccc}\text { A } & \text { NGC 3607 } & 10.54 \pm 0.01 & 9.79 \pm 0.01 & 9.39 \pm 0.01 \\ \text { B } & \text { NGC 3608 } & 11.57 \pm 0.01 & 10.82 \pm 0.01 & 10.44 \pm 0.01 \\ \text { C } & \text { NGC 3599 } & 12.49 \pm 0.01 & 11.83 \pm 0.01 & 11.47 \pm 0.01 \\ \text { D } & \text { NGC 3605 } & 13.12 \pm 0.01 & 12.42 \pm 0.01 & 12.06 \pm 0.01 \\ \text { E } & \text { UGC 6296 } & 13.97 \pm 0.01 & 13.31 \pm 0.01 & 12.96 \pm 0.01\end{array}$

RSCG 41

$\begin{array}{lllll}\text { A } & \text { NGC } 3686 & 11.55 \pm 0.01 & 11.03 \pm 0.01 & 10.76 \pm 0.01\end{array}$ 
Table A.2-Continued

\begin{tabular}{llccc}
\hline \hline & Galaxy & $\begin{array}{c}\text { g' } \\
\text { mag }\end{array}$ & $\begin{array}{c}\text { r' } \\
\text { mag }\end{array}$ & $\begin{array}{c}\text { i' } \\
\text { mag }\end{array}$ \\
\hline B & NGC 3684 & $12.12 \pm 0.01$ & $11.65 \pm 0.01$ & $11.28 \pm 0.02$ \\
C & NGC 3681 & $12.16 \pm 0.01$ & $11.53 \pm 0.01$ & $11.20 \pm 0.01$ \\
D & NGC 3691 & $13.20 \pm 0.01$ & $12.77 \pm 0.01$ & $12.58 \pm 0.01$
\end{tabular}

RSCG 42

$\begin{array}{ccccc}\text { A } & \text { UGC 6583 } & 14.09 \pm 0.01 & 13.65 \pm 0.01 & 13.51 \pm 0.01 \\ \text { B } & \text { KUG 1134+202A } & 14.53 \pm 0.01 & 13.96 \pm 0.01 & 13.71 \pm 0.01 \\ \text { C } & \text { ARK 303 } & 14.98 \pm 0.01 & 14.23 \pm 0.01 & 13.89 \pm 0.01\end{array}$

RSCG 44

$\begin{array}{lllll}\text { A } & \text { NGC } 3842 & 12.67 \pm 0.01 & 11.87 \pm 0.01 & 11.48 \pm 0.01 \\ \text { B } & \text { NGC 3837 } & 13.74 \pm 0.01 & 12.93 \pm 0.01 & 12.55 \pm 0.01 \\ \text { C } & \text { UGC } 6697 & 13.81 \pm 0.01 & 13.43 \pm 0.01 & 13.28 \pm 0.01 \\ \text { D } & \text { NGC 3841 } & 14.30 \pm 0.01 & 13.49 \pm 0.01 & 13.14 \pm 0.01 \\ \text { E } & \text { NGC 3845 } & 14.71 \pm 0.01 & 13.96 \pm 0.01 & 13.62 \pm 0.01\end{array}$

RSCG 45

$\begin{array}{lllll}\text { A } & \text { CGCG 97-101 } & 14.52 \pm 0.01 & 13.73 \pm 0.01 & 13.35 \pm 0.01 \\ \text { B } & \text { CGCG 97-105 } & 14.98 \pm 0.01 & 14.19 \pm 0.01 & 13.84 \pm 0.01 \\ \text { C } & \text { CGCG 97-110 } & 15.21 \pm 0.01 & 14.47 \pm 0.01 & 14.12 \pm 0.01\end{array}$

RSCG 47

$\begin{array}{lcccc}\text { A } & \text { NGC 3995 } & 12.50 \pm 0.01 & 12.27 \pm 0.01 & 12.23 \pm 0.01 \\ \text { B } & \text { NGC 3994 } & 13.10 \pm 0.01 & 12.49 \pm 0.01 & 12.22 \pm 0.01 \\ \text { C } & \text { NGC 3991N } & 13.98 \pm 0.01 & 13.88 \pm 0.01 & 13.98 \pm 0.01 \\ \text { D } & \text { NGC 3991S } & 14.13 \pm 0.01 & 13.73 \pm 0.01 & 13.59 \pm 0.01\end{array}$

RSCG 48

$\begin{array}{llllc}\text { A } & \text { NGC } 4111 & 11.05 \pm 0.01 & 10.44 \pm 0.01 & 9.97 \pm 0.01 \\ \text { B } & \text { NGC 4117 } & 13.43 \pm 0.01 & 12.70 \pm 0.01 & 12.33 \pm 0.01 \\ \text { C } & \text { UGC 7089 } & 13.47 \pm 0.01 & 13.06 \pm 0.01 & 12.83 \pm 0.01\end{array}$

RSCG 50

$\begin{array}{lllll}\text { A } & \text { NGC } 4206 & 12.55 \pm 0.01 & 12.00 \pm 0.01 & 11.70 \pm 0.01\end{array}$ 
Table A.2-Continued

\begin{tabular}{ccccc}
\hline \hline & Galaxy & g' & r' & i' \\
& & mag & mag & mag \\
\hline B & IC 3056 & $15.26 \pm 0.01$ & $14.94 \pm 0.01$ & $14.83 \pm 0.01$ \\
C & IC 3066 & $15.34 \pm 0.01$ & $14.85 \pm 0.01$ & $14.60 \pm 0.01$
\end{tabular}

RSCG 51

$\begin{array}{ccccc}\text { A } & \text { NGC 4274 } & 10.89 \pm 0.01 & 10.09 \pm 0.01 & 9.67 \pm 0.01 \\ \text { B } & \text { NGC 4278 } & 10.71 \pm 0.01 & 9.95 \pm 0.01 & 9.53 \pm 0.01 \\ \text { C } & \text { NGC 4314 } & 10.93 \pm 0.01 & 10.20 \pm 0.01 & 9.82 \pm 0.01 \\ \text { D } & \text { NGC 4283 } & 12.71 \pm 0.01 & 11.96 \pm 0.01 & 11.56 \pm 0.01 \\ \text { E } & \text { NGC 4286 } & 13.93 \pm 0.01 & 13.33 \pm 0.01 & 13.02 \pm 0.01 \\ \text { F } & \text { NGC 4308 } & 13.96 \pm 0.01 & 13.29 \pm 0.01 & 12.95 \pm 0.01 \\ \text { G } & \text { IC 779 } & 14.83 \pm 0.01 & 14.20 \pm 0.01 & 13.89 \pm 0.01 \\ \text { H } & \text { SDSS J122120.10+294255.7 } & 15.89 \pm 0.01 & 15.27 \pm 0.01 & 14.97 \pm 0.01 \\ \text { I } & \text { SDSS J121943.54+293931.7 } & 17.70 \pm 0.02 & 17.09 \pm 0.02 & 16.81 \pm 0.02 \\ \text { RSCG 52 } & & & & \\ \text { A } & \text { CGCG 215-065 } & 14.49 \pm 0.01 & 13.75 \pm 0.01 & 13.42 \pm 0.01 \\ \text { B } & \text { KUG 1218+401A } & 14.68 \pm 0.01 & 14.19 \pm 0.01 & 14.02 \pm 0.01 \\ \text { C } & \text { KUG 1218+401B } & 14.80 \pm 0.01 & 14.38 \pm 0.01 & 14.25 \pm 0.01\end{array}$

RSCG 53

$\begin{array}{lllll}\text { A } & \text { NGC } 4298 & 11.61 \pm 0.01 & 10.98 \pm 0.01 & 10.66 \pm 0.01 \\ \text { B } & \text { NGC 4302 } & 11.93 \pm 0.01 & 11.13 \pm 0.01 & 10.67 \pm 0.01 \\ \text { C } & \text { UGC } 7436 & 14.15 \pm 0.01 & 13.50 \pm 0.01 & 13.19 \pm 0.01\end{array}$

RSCG 54

$\begin{array}{lcccc}\text { A } & \text { NGC } 4382 & 9.48 \pm 0.01 & 8.81 \pm 0.01 & 8.41 \pm 0.01 \\ \text { B } & \text { NGC } 4394 & 11.37 \pm 0.01 & 10.70 \pm 0.01 & 10.36 \pm 0.01 \\ \text { C } & \text { IC } 3292 & 14.65 \pm 0.01 & 13.97 \pm 0.01 & 13.61 \pm 0.01\end{array}$

RSCG 55
A
NGC 4410B
$\begin{array}{lll}13.78 \pm 0.01 & 12.97 \pm 0.01 & 12.58 \pm 0.01\end{array}$
B NGC 4410A
$\begin{array}{lll}13.85 \pm 0.01 & 13.06 \pm 0.01 & 12.71 \pm 0.01\end{array}$
C
NGC 4410C
$14.63 \pm 0.01$
$13.78 \pm 0.01 \quad 13.40 \pm 0.01$ 
Table A.2 - Continued

\begin{tabular}{ccccc}
\hline \hline Galaxy & $\begin{array}{c}\text { g' } \\
\text { mag }\end{array}$ & $\begin{array}{c}\text { r' } \\
\text { mag }\end{array}$ & $\begin{array}{c}\text { i' } \\
\text { mag }\end{array}$ \\
\hline D & NGC 4410D & $15.20 \pm 0.01$ & $14.55 \pm 0.01$ & $14.29 \pm 0.01$ \\
E & NGC 4410F & $16.63 \pm 0.01$ & $16.26 \pm 0.01$ & $16.18 \pm 0.01$ \\
RSCG 56 & & & \\
A & NGC 4440 & $12.31 \pm 0.01$ & $11.58 \pm 0.01$ & $11.23 \pm 0.01$ \\
B & NGC 4431 & $13.62 \pm 0.01$ & $12.92 \pm 0.01$ & $12.58 \pm 0.01$ \\
C & NGC 4436 & $13.76 \pm 0.01$ & $13.09 \pm 0.01$ & $12.76 \pm 0.01$ \\
D & IC 3349 & $14.78 \pm 0.01$ & $14.10 \pm 0.01$ & $13.79 \pm 0.01$ \\
E & IC 3363 & $15.20 \pm 0.01$ & $14.60 \pm 0.01$ & $14.30 \pm 0.01$
\end{tabular}

\section{RSCG 57}

$\begin{array}{ccccc}\text { A } & \text { NGC 4442 } & 10.94 \pm 0.01 & 10.18 \pm 0.01 & 9.79 \pm 0.01 \\ \text { B } & \text { NGC 4417 } & 11.66 \pm 0.01 & 10.92 \pm 0.01 & 10.54 \pm 0.01 \\ \text { C } & \text { NGC 4424 } & 11.94 \pm 0.01 & 11.39 \pm 0.01 & 11.09 \pm 0.01 \\ \text { D } & \text { NGC 4451 } & 12.97 \pm 0.01 & 12.39 \pm 0.01 & 12.10 \pm 0.01 \\ \text { E } & \text { NGC 4445 } & 13.20 \pm 0.01 & 12.49 \pm 0.01 & 12.12 \pm 0.01 \\ \text { F } & \text { IC 3412 } & 15.39 \pm 0.01 & 15.00 \pm 0.01 & 14.82 \pm 0.01 \\ \text { G } & \text { VCC 1078 } & 15.82 \pm 0.01 & 15.42 \pm 0.01 & 15.23 \pm 0.01\end{array}$

RSCG 58
A NGC 4435
$11.20 \pm 0.01$
$10.44 \pm 0.0$
$10.05 \pm 0.01$
B NGC 4458
$12.66 \pm 0.01$
$11.97 \pm 0.01$
$11.59 \pm 0.01$
C IC 3393
$14.76 \pm 0.01$
$14.15 \pm 0.01$
$13.86 \pm 0.01$

RSCG 59
A NGC 4459
$11.29 \pm 0.02$
$10.50 \pm 0.01$
$10.10 \pm 0.01$
B NGC 4474
$12.35 \pm 0.01$
$11.64 \pm 0.01$
$11.27 \pm 0.01$
C $\quad$ NGC 4468
$13.57 \pm 0.01$
$12.89 \pm 0.01$
$12.54 \pm 0.01$

RSCG 60
A NGC 4469
$11.62 \pm 0.01$
$10.87 \pm 0.01$
$10.47 \pm 0.01$
B NGC 4483
$12.77 \pm 0.01$
$12.03 \pm 0.01$
$11.66 \pm 0.01$
C UGC 7590
$14.00 \pm 0.01$
$13.71 \pm 0.01$
$13.54 \pm 0.01$ 
Table A.2 - Continued

\begin{tabular}{ccccc}
\hline \hline Galaxy & g' & r' & i' \\
& mag & mag & mag \\
\hline $\mathrm{D}^{\dagger}$ & UGC 7596 & $14.74 \pm 0.01$ & $14.31 \pm 0.01$ & $14.09 \pm 0.01$
\end{tabular}

\section{RSCG 61}

$\begin{array}{ccccc}\text { A } & \text { M 87 } & 9.99 \pm 0.01 & 9.19 \pm 0.01 & 8.78 \pm 0.01 \\ \text { B } & \text { NGC 4478 } & 11.84 \pm 0.01 & 11.12 \pm 0.01 & 10.75 \pm 0.01 \\ \text { C } & \text { NGC 4486A } & 12.46 \pm 0.01 & 11.91 \pm 0.01 & 11.56 \pm 0.01 \\ \text { D } & \text { NGC 4486B } & 13.83 \pm 0.01 & 13.03 \pm 0.01 & 12.64 \pm 0.01\end{array}$

RSCG 62

$\begin{array}{ccccc}\text { A } & \text { NGC } 4497 & 12.84 \pm 0.02 & 12.17 \pm 0.02 & 11.85 \pm 0.01 \\ \text { B } & \text { NGC 4491 } & 13.00 \pm 0.02 & 12.37 \pm 0.02 & 12.04 \pm 0.01 \\ \text { C } & \text { IC } 3461 & 15.03 \pm 0.02 & 14.39 \pm 0.02 & 14.07 \pm 0.01 \\ \text { D } & \text { IC } 3466 & 15.15 \pm 0.02 & 14.81 \pm 0.02 & 14.68 \pm 0.01 \\ \text { E } & \text { IC } 3446 & 15.54 \pm 0.02 & 15.19 \pm 0.02 & 15.02 \pm 0.02\end{array}$

RSCG 63

$\begin{array}{ccccc}\text { A } & \text { NGC 4552 } & 10.90 \pm 0.01 & 10.11 \pm 0.01 & 9.72 \pm 0.01 \\ \text { B } & \text { NGC 4550 } & 12.06 \pm 0.01 & 11.34 \pm 0.01 & 10.99 \pm 0.01 \\ \text { C } & \text { NGC 4551 } & 12.63 \pm 0.01 & 11.87 \pm 0.01 & 11.49 \pm 0.01 \\ \text { D } & \text { IC 3540 } & 14.26 \pm 0.01 & 13.68 \pm 0.01 & 13.40 \pm 0.01\end{array}$

RSCG 64

$\begin{array}{lllll}\text { A } & \text { NGC 4615 } & 13.56 \pm 0.01 & 13.14 \pm 0.01 & 12.99 \pm 0.01 \\ \text { B } & \text { NGC 4614 } & 13.83 \pm 0.01 & 13.12 \pm 0.01 & 12.80 \pm 0.01 \\ \text { C } & \text { NGC 4613 } & 15.09 \pm 0.01 & 14.43 \pm 0.01 & 14.14 \pm 0.01\end{array}$

RSCG 65
A NGC 4649
$9.93 \pm 0.01$
$9.12 \pm 0.01$
$8.69 \pm 0.01$
B NGC 4621
$10.77 \pm 0.01$
$9.98 \pm 0.01$
$9.56 \pm 0.01$
C NGC 4647
$11.51 \pm 0.01$
$10.91 \pm 0.01$
$10.60 \pm 0.01$
D NGC 4638
$11.70 \pm 0.01$
$10.97 \pm 0.01$
$10.60 \pm 0.01$
E IC 3653
$14.09 \pm 0.01$
$13.35 \pm 0.01$
$12.99 \pm 0.01$
F IC 3652
$14.18 \pm 0.01$
$13.52 \pm 0.01$
$13.18 \pm 0.01$ 
Table A.2-Continued

\begin{tabular}{ccccc}
\hline \hline & Galaxy & $\begin{array}{c}\text { g' } \\
\text { mag }\end{array}$ & $\begin{array}{c}\text { r' } \\
\text { mag }\end{array}$ & $\begin{array}{c}\text { i' } \\
\text { mag }\end{array}$ \\
\hline G & IC 3665 & $14.99 \pm 0.01$ & $14.44 \pm 0.01$ & $14.17 \pm 0.01$ \\
RSCG 66 & & & \\
A & NGC 4654 & $10.81 \pm 0.01$ & $10.30 \pm 0.01$ & $10.04 \pm 0.01$ \\
B & NGC 4639 & $11.86 \pm 0.01$ & $11.27 \pm 0.01$ & $10.96 \pm 0.01$ \\
C & VCC 1931 & $15.74 \pm 0.01$ & $15.47 \pm 0.01$ & $15.35 \pm 0.01$
\end{tabular}

RSCG $67^{* *}$

$\begin{array}{lllll}\text { A } & \text { NGC 4874 } & 12.55 \pm 0.01 & 11.73 \pm 0.01 & 11.33 \pm 0.01 \\ \text { B } & \text { NGC 4871 } & 14.75 \pm 0.01 & 13.94 \pm 0.01 & 13.59 \pm 0.01 \\ \text { C } & \text { NGC 4872 } & 14.91 \pm 0.02 & 14.10 \pm 0.02 & 13.74 \pm 0.02\end{array}$

RSCG $68^{* *}$
A $\quad$ NGC 4889
$13.02 \pm 0.01 \quad 12.18 \pm 0.01$
$11.79 \pm 0.01$
B NGC 4898W
$14.26 \pm 0.01$
$13.47 \pm 0.01$
$13.11 \pm 0.01$
C $\quad$ NGC 4886
$14.63 \pm 0.01$
$13.87 \pm 0.01$
$13.50 \pm 0.01$

\section{RSCG 69}

$\begin{array}{lllll}\text { A } & \text { IC } 867 & 14.28 \pm 0.01 & 13.67 \pm 0.01 & 13.39 \pm 0.01 \\ \text { B } & \text { IC } 868 & 14.39 \pm 0.01 & 13.59 \pm 0.01 & 13.24 \pm 0.01 \\ \text { C } & \text { IC } 870 & 14.53 \pm 0.01 & 13.93 \pm 0.01 & 13.68 \pm 0.01\end{array}$

RSCG 72
A IC 962
$13.70 \pm 0.01$
$13.04 \pm 0.01$
$12.75 \pm 0.01$
B CGCG 74-014
$14.75 \pm 0.01 \quad 14.06 \pm 0.01$
$13.74 \pm 0.01$
C CGCG 74-016
$15.20 \pm 0.01$
$14.81 \pm 0.01$
$14.66 \pm 0.01$

RSCG 73
A NGC 5423
$13.43 \pm 0.0$
$12.63 \pm 0.01$
$12.26 \pm 0.01$
B CGCG 074-058
$14.96 \pm 0.01$
$14.18 \pm 0.01$
$13.83 \pm 0.01$
C CGCG 074-062
$15.56 \pm 0.01$
$14.85 \pm 0.01$
$14.53 \pm 0.01$

RSCG 74
A NGC 5504
$13.50 \pm 0.0$
$12.98 \pm 0.01$
$12.75 \pm 0.01$
B NGC 5504B
$15.06 \pm 0.01$
$14.49 \pm 0.01$
$14.25 \pm 0.01$ 
Table A.2-Continued

\begin{tabular}{lcccc}
\hline \hline & Galaxy & $\begin{array}{c}\text { g' } \\
\text { mag }\end{array}$ & $\begin{array}{c}\text { r' } \\
\text { mag }\end{array}$ & $\begin{array}{c}\text { i' } \\
\text { mag }\end{array}$ \\
\hline C & NGC 5504C & $15.59 \pm 0.01$ & $15.36 \pm 0.01$ & $15.29 \pm 0.01$ \\
RSCG 75 & & & \\
A & UGC 9521 & $13.99 \pm 0.01$ & $13.25 \pm 0.01$ & $12.90 \pm 0.01$ \\
B & UGC 9523 & $14.08 \pm 0.01$ & $13.28 \pm 0.01$ & $12.92 \pm 0.01$ \\
C & CGCG 76-040 & $15.14 \pm 0.01$ & $14.35 \pm 0.01$ & $14.00 \pm 0.01$
\end{tabular}

RSCG 76
A NGC 5852
$13.86 \pm 0.01$
$13.03 \pm 0.01$
$12.64 \pm 0.01$
B NGC 5851
$14.42 \pm 0.01$
$13.78 \pm 0.01$
$13.50 \pm 0.01$
C CGCG 77-007 $\quad 15.51 \pm 0.01$
$14.90 \pm 0.01$
$14.63 \pm 0.01$

RSCG 78
A NGC 6962
$12.07 \pm 0.02$
$11.36 \pm 0.01$
$11.01 \pm 0.01$
B NGC 6964
$13.19 \pm 0.02$
$12.41 \pm 0.01$
$12.04 \pm 0.01$
C $\quad$ NGC 6959
$13.89 \pm 0.02$
$13.21 \pm 0.01$
$12.93 \pm 0.01$
D N NGC 6961
$14.60 \pm 0.02$
$13.82 \pm 0.01$
$13.46 \pm 0.01$

RSCG 83
A NGC 7463
$12.87 \pm 0.02$
$12.48 \pm 0.01$
$12.30 \pm 0.01$
B NGC 7465
$12.87 \pm 0.02$
$12.25 \pm 0.01$
$11.98 \pm 0.01$
C NGC 7464
$14.37 \pm 0.02$
$14.22 \pm 0.01$
$14.20 \pm 0.01$

RSCG 84
A NGC 7532
$13.86 \pm 0.01$
$13.42 \pm 0.01$
$13.24 \pm 0.01$
B NGC 7534
$14.29 \pm 0.01$
$14.04 \pm 0.01$
$13.98 \pm 0.01$
C NGC 7533
$14.62 \pm 0.01$
$14.16 \pm 0.01$
$13.97 \pm 0.01$

RSCG 85
A UGC 12545
$14.59 \pm 0.02$
$14.15 \pm 0.01$
$13.99 \pm 0.01$
B UGC 12546
$14.62 \pm 0.02$
$14.06 \pm 0.01$
$13.83 \pm 0.01$
C UGC 12543
$15.18 \pm 0.02$
$14.71 \pm 0.01$
$14.50 \pm 0.01$

RSCG 86

A $\quad$ UGC 12716a $\quad 13.28 \pm 0.02 \quad 12.43 \pm 0.02 \quad 12.04 \pm 0.01$ 
Table A.2-Continued

\begin{tabular}{ccccc}
\hline \hline & Galaxy & g' & r' & i' \\
& & mag & mag & mag \\
\hline B & IC 5342 & $14.85 \pm 0.02$ & $14.03 \pm 0.01$ & $13.66 \pm 0.01$ \\
C & UGC 12716b & $15.00 \pm 0.02$ & $14.16 \pm 0.02$ & $13.78 \pm 0.02$ \\
D & 2MASX J23383626+2701467 & $15.31 \pm 0.02$ & $14.46 \pm 0.01$ & $14.07 \pm 0.01$ \\
\hline
\end{tabular}

Note. - ${ }^{\dagger}$ Galaxy added by us. ${ }^{* *}$ Groups embedded in Perseus (RSCG 21) or Coma (RSCGs $67 \& 68)$. These were excluded from our analysis. 
Appendix B

Acronyms 
Table B.1. Acronyms

\begin{tabular}{cc}
\hline \hline Acronym & Definition \\
\hline FLS & First Look Survey \\
PAH & polycyclic aromatic hydrocarbon \\
SINGS & Spitzer Infrared Nearby Galaxy Survey \\
HCG & Hickson compact group \\
RSCG & Redshift Survey compact group \\
LVL & Local Volume Legacy \\
CMD & color-magnitude diagram \\
IRAC & Infrared Array Camera \\
SSFR & specific star formation rate \\
SED & spectral energy distribution \\
KS & Kolmogorov-Smirnov \\
EW & equivalent width \\
CG & compact group \\
ISM & interstellar medium \\
NYU VAGC & New York University Value-Added Galaxy Catalog \\
SDSS & Sloan Digital Sky Survey \\
AGN & active galactic nucleus or nuclei \\
SFR & star formation rate \\
IRAS & Infrared Astronomical Satellite \\
CfA & Center for Astrophysics \\
UZC & Updated Zwicky Catalog \\
IGM & intragroup medium \\
NED & NASA Extragalactic Database \\
CDF & cumulative distribution function \\
IRAF & Image Reduction and Analysis Facility \\
2MASS & Two Micron All Sky Survey \\
IRS & Infrared Spectrograph \\
MIRTG & Mid-IR Transition Galaxy \\
GBT & Green Bank Telescope \\
NVSS & NRAO VLA Sky Survey \\
\hline &
\end{tabular}




\section{References}

Adelman-McCarthy, J. K., Agüeros, M. A., Allam, S. S., et al. 2008, ApJS, 175, 297

Aihara, H., Allende Prieto, C., An, D., et al. 2011, ApJS, 193, 29

Allam, S., Assendorp, R., Longo, G., \& Richter, G. 1995, Planet. Space Sci., 43, 1371

Allam, S. S., \& Tucker, D. L. 2000, Astronomische Nachrichten, 321, 101

Barton, E., Geller, M., Ramella, M., Marzke, R. O., \& da Costa, L. N. 1996, AJ, 112, 871

Bell, E. F., McIntosh, D. H., Katz, N., \& Weinberg, M. D. 2003, ApJS, 149, 289

Bell, E. F., Wolf, C., Meisenheimer, K., et al. 2004, ApJ, 608, 752

Bernardi, M., Renzini, A., da Costa, L. N., et al. 1998, ApJ, 508, L143

Blanton, M. R., \& Berlind, A. A. 2007, ApJ, 664, 791

Blanton, M. R., Lupton, R. H., Schlegel, D. J., et al. 2005a, ApJ, 631, 208

Blanton, M. R., \& Moustakas, J. 2009, ARA\&A, 47, 159

Blanton, M. R., \& Roweis, S. 2007, AJ, 133, 734

Blanton, M. R., Dalcanton, J., Eisenstein, D., et al. 2001, AJ, 121, 2358 
Blanton, M. R., Schlegel, D. J., Strauss, M. A., et al. 2005b, AJ, 129, 2562

Borthakur, S., Yun, M. S., \& Verdes-Montenegro, L. 2010, ApJ, 710, 385

Boselli, A., Mendes de Oliveira, C., Balkowski, C., Cayatte, V., \& Casoli, F. 1996, A\&A, 314, 738

Bressan, A., Silva, L., \& Granato, G. L. 2002, A\&A, 392, 377

Cluver, M. E., Appleton, P. N., Boulanger, F., et al. 2010, ApJ, 710, 248

Cluver, M. E., Appleton, P. N., Ogle, P., et al. 2013, ApJ, 765, 93

Coenda, V., Muriel, H., \& Martínez, H. J. 2012, A\&A, 543, A119

Condon, J. J., Cotton, W. D., Greisen, E. W., et al. 1998, AJ, 115, 1693

Cooper, M. C., Griffith, R. L., Newman, J. A., et al. 2012, MNRAS, 419, 3018

Cortese, L., Gavazzi, G., Boselli, A., et al. 2006, A\&A, 453, 847

Coziol, R., Brinks, E., \& Bravo-Alfaro, H. 2004, AJ, 128, 68

Coziol, R., \& Plauchu-Frayn, I. 2007, AJ, 133, 2630

Daddi, E., Dickinson, M., Morrison, G., et al. 2007, ApJ, 670, 156

Dale, D. A., Gil de Paz, A., Gordon, K. D., et al. 2007, ApJ, 655, 863

Dale, D. A., Cohen, S. A., Johnson, L. C., et al. 2009, ApJ, 703, 517

de la Rosa, I. G., de Carvalho, R. R., Vazdekis, A., \& Barbuy, B. 2007, AJ, 133, 330

Dekel, A., \& Birnboim, Y. 2006, MNRAS, 368, 2

Deng, X.-F., He, J.-Z., \& Wu, P. 2008, A\&A, 484, 355 
Desjardins, T. D., Gallagher, S. C., Tzanavaris, P., et al. 2013, ApJ, 763, 121

Desjardins, T. D., Gallagher, S. C., Hornschemeier, A. E., et al. submitted, ApJ

Dressler, A. 1980, ApJ, 236, 351

Faber, S. M., Willmer, C. N. A., Wolf, C., et al. 2007, ApJ, 665, 265

Focardi, P., \& Kelm, B. 2002, A\&A, 391, 35

Fuse, C., \& Broming, E. 2013, ApJ, 764, 175

Gallagher, S. C., Johnson, K. E., Hornschemeier, A. E., Charlton, J. C., \& Hibbard, J. E. 2008, ApJ, 673, 730

Gallagher, S. C., Durrell, P. R., Elmegreen, D. M., et al. 2010, AJ, 139, 545

Gao, Y., \& Solomon, P. M. 1999, ApJ, 512, L99

Giovanelli, R., \& Haynes, M. P. 1985, ApJ, 292, 404

Gobat, R., Rosati, P., Strazzullo, V., et al. 2008, A\&A, 488, 853

Granato, G. L., Lacey, C. G., Silva, L., et al. 2000, ApJ, 542, 710

Gunn, J. E., \& Gott, III, J. R. 1972, ApJ, 176, 1

Haynes, M. P., \& Giovanelli, R. 1984, AJ, 89, 758

Hickson, P. 1982, ApJ, 255, 382

Hickson, P., Mendes de Oliveira, C., Huchra, J. P., \& Palumbo, G. G. 1992, ApJ, 399,353

Hogg, D. W., Blanton, M. R., Brinchmann, J., et al. 2004, ApJ, 601, L29 
Iglesias-Páramo, J., \& Vílchez, J. M. 1999, ApJ, 518, 94

Jackson, D. C., Cannon, J. M., Skillman, E. D., et al. 2006, ApJ, 646, 192

Jenkins, L. P., Hornschemeier, A. E., Mobasher, B., Alexander, D. M., \& Bauer, F. E. 2007, ApJ, 666, 846

Johnson, K. E., Hibbard, J. E., Gallagher, S. C., et al. 2007, AJ, 134, 1522

Kauffmann, G., White, S. D. M., Heckman, T. M., et al. 2004, MNRAS, 353, 713

Kennicutt, Jr., R. C., \& Kent, S. M. 1983, AJ, 88, 1094

Kennicutt, Jr., R. C., Armus, L., Bendo, G., et al. 2003, PASP, 115, 928

Konstantopoulos, I. S., Gallagher, S. C., Fedotov, K., et al. 2010, ApJ, 723, 197

—. 2012, ApJ, 745, 30

Konstantopoulos, I. S., Maybhate, A., Charlton, J. C., et al. 2013, ApJ, 770, 114

Lee, B. C., Allam, S. S., Tucker, D. L., et al. 2004, AJ, 127, 1811

Lenkic, L., Desjardins, T., Gallagher, S., et al. in prep

Leon, S., Combes, F., \& Menon, T. K. 1998, A\&A, 330, 37

Lewis, I., Balogh, M., De Propris, R., et al. 2002, MNRAS, 334, 673

Mahajan, S., Haines, C. P., \& Raychaudhury, S. 2010, MNRAS, 404, 1745

Mamon, G. A. 1994, in Clusters of Galaxies, ed. F. Durret, A. Mazure, \& J. Tran Thanh Van, 291

Martin, D. C., Wyder, T. K., Schiminovich, D., et al. 2007, ApJS, 173, 342 
Martínez, M. A., del Olmo, A., Coziol, R., \& Focardi, P. 2008, ApJ, 678, L9

Martínez, M. A., Del Olmo, A., Coziol, R., \& Perea, J. 2010, AJ, 139, 1199

Martinez-Badenes, V., Lisenfeld, U., Espada, D., et al. 2012, A\&A, 540, A96

McConnachie, A. W., Ellison, S. L., \& Patton, D. R. 2008, MNRAS, 387, 1281

Mendel, J. T., Ellison, S. L., Simard, L., Patton, D. R., \& McConnachie, A. W. 2011, MNRAS, 418, 1409

Mendes de Oliveira, C., Amram, P., Plana, H., \& Balkowski, C. 2003, AJ, 126, 2635

Mendes de Oliveira, C., Coelho, P., González, J. J., \& Barbuy, B. 2005, AJ, 130, 55

Minezaki, T., Yoshii, Y., Kobayashi, Y., et al. 2006, ApJ, 643, L5

Momose, R., Koda, J., Kennicutt, Jr., R. C., et al. 2013, ApJ, 772, L13

Monnier Ragaigne, D., van Driel, W., Schneider, S. E., Jarrett, T. H., \& Balkowski, C. 2003, A\&A, 405, 99

Moore, B., Lake, G., \& Katz, N. 1998, ApJ, 495, 139

Moustakas, J., \& Kennicutt, Jr., R. C. 2006, ApJS, 164, 81

Mulchaey, J. S. 2000, ARA\&A, 38, 289

Nakamura, O., Fukugita, M., Brinkmann, J., \& Schneider, D. P. 2004, AJ, 127, 2511

Noeske, K. G., Faber, S. M., Weiner, B. J., et al. 2007, ApJ, 660, L47

Padmanabhan, N., Schlegel, D. J., Finkbeiner, D. P., et al. 2008, ApJ, 674, 1217

Pahre, M. A., Ashby, M. L. N., Fazio, G. G., \& Willner, S. P. 2004, ApJS, 154, 235 
Palumbo, G. G. C., Saracco, P., Hickson, P., \& Mendes de Oliveira, C. 1995, AJ, 109, 1476

Panuzzo, P., Bressan, A., Granato, G. L., Silva, L., \& Danese, L. 2003, A\&A, 409, 99

Plauchu-Frayn, I., Del Olmo, A., Coziol, R., \& Torres-Papaqui, J. P. 2012, A\&A, 546, A48

Ponman, T. J., Bourner, P. D. J., Ebeling, H., \& Böhringer, H. 1996, MNRAS, 283, 690

Postman, M., \& Geller, M. J. 1984, ApJ, 281, 95

Prandoni, I., Iovino, A., \& MacGillivray, H. T. 1994, AJ, 107, 1235

Proctor, R. N., Forbes, D. A., Hau, G. K. T., et al. 2004, MNRAS, 349, 1381

Rasmussen, J., Ponman, T. J., Verdes-Montenegro, L., Yun, M. S., \& Borthakur, S. 2008, MNRAS, 388, 1245

Reines, A. E., Johnson, K. E., \& Goss, W. M. 2008, AJ, 135, 2222

Ribeiro, A. L. B., de Carvalho, R. R., Capelato, H. V., \& Zepf, S. E. 1998, ApJ, 497, 72

Rupen, M. P., Hibbard, J. E., \& Bunker, K. 2001, in Astronomical Society of the Pacific Conference Series, Vol. 240, Gas and Galaxy Evolution, ed. J. E. Hibbard, M. Rupen, \& J. H. van Gorkom

Sánchez-Blázquez, P., Gorgas, J., Cardiel, N., \& González, J. J. 2006, A\&A, 457, 809

Schlegel, D. J., Finkbeiner, D. P., \& Davis, M. 1998, ApJ, 500, 525 
Shakhbazyan, R. K. 1973, Astrofizika, 9, 495

Silva, L. 1999, PhD thesis, SISSA - Trieste, Italy

Silva, L. 2009, in Revista Mexicana de Astronomia y Astrofisica Conference Series, Vol. 37, Revista Mexicana de Astronomia y Astrofisica Conference Series, 83-93

Silva, L., Granato, G. L., Bressan, A., \& Danese, L. 1998, ApJ, 509, 103

Silva, L., Granato, G. L., Bressan, A., \& Panuzzo, P. 2003, in Revista Mexicana de Astronomia y Astrofisica Conference Series, Vol. 17, Revista Mexicana de Astronomia y Astrofisica Conference Series, ed. V. Avila-Reese, C. Firmani, C. S. Frenk, \& C. Allen, 93-93

Skrutskie, M. F., Cutri, R. M., Stiening, R., et al. 2006, AJ, 131, 1163

Smith, B. J., Struck, C., Hancock, M., et al. 2007, AJ, 133, 791

Sohn, J., Hwang, H. S., Lee, M. G., Lee, G.-H., \& Lee, J. C. 2013, ApJ, 771, 106

Strateva, I., Ivezić, Ž., Knapp, G. R., et al. 2001, AJ, 122, 1861

Thibert, N., Desjardins, T., Gallagher, S., et al. in prep

Thilker, D. A., Bianchi, L., Schiminovich, D., et al. 2010, ApJ, 714, L171

Toribio, M. C., Solanes, J. M., Giovanelli, R., Haynes, M. P., \& Martin, A. M. 2011, ApJ, 732, 93

Torres-Flores, S., Mendes de Oliveira, C., Amram, P., et al. 2010, A\&A, 521, A59

Torres-Flores, S., Mendes de Oliveira, C., Plana, H., Amram, P., \& Epinat, B. 2013, MNRAS, 432, 3085 
Tzanavaris, P., Hornschemeier, A. E., Gallagher, S. C., et al. 2010, ApJ, 716, 556

van Dokkum, P. G. 2005, AJ, 130, 2647

Vega, O., Silva, L., Panuzzo, P., et al. 2005, MNRAS, 364, 1286

Verdes-Montenegro, L., Del Olmo, A., Yun, M. S., \& Perea, J. 2005, A\&A, 430, 443

Verdes-Montenegro, L., Yun, M. S., Perea, J., del Olmo, A., \& Ho, P. T. P. 1998, ApJ, 497, 89

Verdes-Montenegro, L., Yun, M. S., Williams, B. A., et al. 2001, A\&A, 377, 812

Walker, L. M., Johnson, K. E., Gallagher, S. C., et al. 2012, AJ, 143, 69

-. 2010, AJ, 140, 1254

Walker, L. M., Butterfield, N., Johnson, K., et al. 2013, ApJ, 775, 129

Weinzirl, T., Jogee, S., Conselice, C. J., et al. 2011, ApJ, 743, 87

Williams, B. A., McMahon, P. M., \& van Gorkom, J. H. 1990, in NASA Conference Publication, Vol. 3098, NASA Conference Publication, ed. J. W. Sulentic, W. C. Keel, \& C. M. Telesco, 93-98

Wilman, D. J., Pierini, D., Tyler, K., et al. 2008, ApJ, 680, 1009

Wyder, T. K., Martin, D. C., Schiminovich, D., et al. 2007, ApJS, 173, 293 